Elsevier Editorial System(tm) for Steroids Manuscript Draft

Manuscript Number: STEROIDS-D-19-00155R1

Title: Stereocontrolled Synthesis of the Four Possible 3-Methoxy and 3Benzyloxy-16-Triazolyl-methyl-estra-17-ol Hybrids and their Antiproliferative Activities

Article Type: Regular Article

Keywords: 3-methoxy- and 3-benzyloxy-16-azidomethylestra-1,3,5(10)triene-17-ols; 1,3-dipolar cycloaddition, 4'substituted-steroid triazoles; cytotoxic activity

Corresponding Author: Professor Gyula Schneider,

Corresponding Author's Institution: University of Szeged

First Author: Anita Kiss

Order of Authors: Anita Kiss; János Wölfling; Erzsébet Mernyák; Éva Frank; Zsanett Benke; Seyyed Ashkan Senobar Tahaei; István Zupkó; Sándor Mahó; Gyula Schneider

Abstract: The four possible isomers of each of 3-methoxy- and 3benzyloxyestra-1,3,5(10)-trien-17-ols (5-8 and 9-12) were converted through 16-p-tosyloxymethyl- or 16-bromomethyl derivatives into their 3methoxy- and 3-benzyloxy-16-azidomethylestra(1,3,5(10)-triene derivatives (13-16 and 17-20). The regioselective Cu(I)-catalyzed 1,3-dipolar cycloaddition of these compounds with different terminal alkynes afforded novel 1,4-disubstituted diastereomers (21a-f, 22a-f, 23a-f, 24a-f and 25a-f, 26a-f, 27a-f, 28a-f). The antiproliferative activities of the structurally related triazoles were determined in vitro with the microculture tetrazolium assay on four malignant human cell lines of gynecological origin (Hela, SiHa, MCF-7 and MDA-MB-231). 
Response to Reviewer's comments:

Reviewer 1:

1. Graphical abstract have been uploaded.

2. Absolute configuration of compounds $\mathbf{5}$ and $\mathbf{8}$ has been unambigously discussed in Ref. $\mathbf{5}$ and 6 , and in the Figure 1. of the present manuscript.

3. Corrected.

4. Corrected.

Reviewer 2:

1. Graphical abstract have been uploaded.

2. Corrected.

3. Copies of NMR spectra have been uploaded.

4. Corrected, and highlighted in yellow.

Thank you for your reviews. 


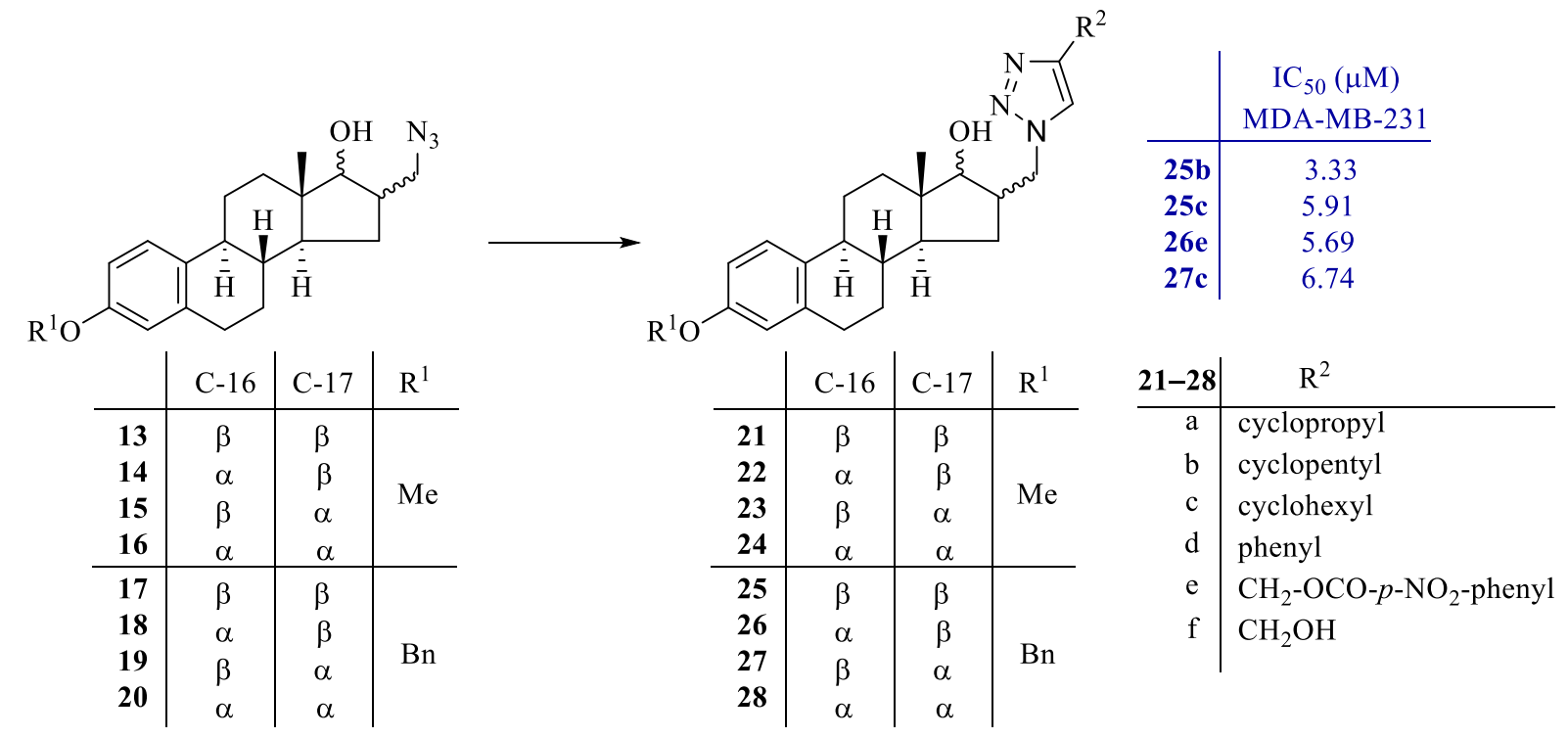


Highlights (for review)

Highlights

1. Synthesis of 3-methoxy- and 3-benzyloxy-16-azidomethylestra(1,3,5(10)-trienes.

2. CUAAC reaction of 16-azidomethyl steroidal compounds with different terminal alkynes.

3. Substantial antiproliferative activity for 3-benzyl-16-triazolylmethylene derivatives. 


\title{
Stereocontrolled Synthesis of the Four Possible 3-Methoxy and 3-Benzyloxy- 16-Triazolyl-methyl-estra-17-ol Hybrids and their Antiproliferative Activities
}

\author{
Anita Kiss ${ }^{a}$, János Wölfling ${ }^{a}$, Erzsébet Mernyák ${ }^{a}$, Éva Frank ${ }^{a}$, Zsanett Benke ${ }^{b}$, Seyyed \\ Ashkan Senobar Tahaei ${ }^{c}$, István Zupkóo ${ }^{\text {,d }}$, Sándor Mahó ${ }^{\mathrm{e}}$, Gyula Schneider $^{\text {** }}$ \\ aa Department of Organic Chemistry, University of Szeged, Dóm tér 8., H-6720 Szeged, Hungary \\ ${ }^{\mathrm{b}}$ Institute of Pharmaceutical Chemistry, University of Szeged, H-6720, Eötvös u. 6., H-6720 Szeged. Hungary \\ ${ }^{c}$ Department of Pharmacodynamics and Biopharmacy, University of Szeged, Eötvös u. 6., H-6720 Szeged, Hungary \\ ${ }^{\mathrm{d}}$ Interdisciplinary Centre for Natural Products, University of Szeged, Eötvös u. 6., H-6720 Szeged, Hungary \\ ${ }^{e}$ Chemical Works of Gedeon Richter Plc., Gyömröi út 19-21., H-1103 Budapest, Hungary
}

\begin{abstract}
The four possible isomers of each of 3-methoxy- and 3-benzyloxyestra-1,3,5(10)trien-17-ols (5-8 and 9-12) were converted through 16-p-tosyloxymethyl- or 16-bromomethyl derivatives into their 3-methoxy- and 3-benzyloxy-16-azidomethylestra(1,3,5(10)-triene derivatives (13-16 and 17-20). The regioselective $\mathrm{Cu}(\mathrm{I})$-catalyzed 1,3-dipolar cycloaddition of these compounds with different terminal alkynes afforded novel 1,4-disubstituted diastereomers (21a-f, 22a-f, 23a-f, 24a-f and 25a-f, 26a-f, 27a-f, 28a-f). The antiproliferative activities of the structurally related triazoles were determined in vitro with the microculture tetrazolium assay on four malignant human cell lines of gynecological origin (Hela, SiHa, MCF-7 and MDA-MB231).
\end{abstract}

Keywords: 3-methoxy- and 3-benzyloxy-16-azidomethylestra-1,3,5(10)-triene-17-ols; 1,3-dipolar cycloaddition, 4 'substituted-steroid triazoles; cytotoxic activity

\section{Introduction}



due to significant biological properties and numerous therapeutic effects of the basic compound. Steroids have become ideal synthons for the development of diverse conjugates due to their rigid framework and potential for varying levels of functionalization, broad biological activity profile and their ability to penetrate the cell membranes and bind to specific hormonal receptors [1-3].

The place, length and orientation of the linkers between the two parts of the hybrids stems unequivocally from the method of their synthesis. The literature provides a large number of methods to introduce the linker onto the sterane skeleton. The effect of the length and character of the linker are very often discussed [4]. However, only limited information is available with respect to the steric effect of the linkers on biological properties. As concerns the 16-substituted estrogenes, usually the $16 \alpha$-substituted-17 $\beta$-hydroxy compounds have been studied. The biological activity has generally not been studied for the whole isomer series [5].

In the 16-substituted 17-hydroxysteroids, the two chiral centres permit four stereochemical modifications. Since availability of the complete series of isomers would permit a number of interesting comparative examinations.

We have previously reported the preparation and configurational assignment of the four possible isomers of the 3-methoxy- and 3-benzyloxy-16-hydroxymethyl-estra-1,3,5(10)-trien-17ol derivatives (5a-8a and 9a-12a) [6-8]. Treatment of 3-methoxy- and 3-benzyloxyestra-16hydroxymethylidene-estra-1,3,5(10)-trien-17-ones (2 and 4). The C-16 formyl compounds were reduced with $\mathrm{KBH}_{4}$ in methanol yielding a mixture of three $(\mathbf{5 a}-7 \mathbf{a}$ and $9 \mathbf{a}-\mathbf{1 1 a})$ of the four possible isomers of each of the 3-methoxy- and 3-benzyloxy-16-hydroxymethylestra-1,3,5(10)trien-17-ol isomers in a ratio of 50:45:5 in 94\% yield [6,8 ]. The fourth isomers (8a and 12a) were prepared from 16 $\alpha$-acetoxymethyl-17 $\beta$-toluenesulfonate mixed esters $\mathbf{6 d}$ and 10d, respectively, by neighbouring group participation during solvolysis in aqueous $\mathrm{AcOH}$. The structures of the isomers were confirmed unambiguously by their IR, ${ }^{1} \mathrm{H}$ and ${ }^{13} \mathrm{C}$ NMR spectra (Scheme 1) [7,8].

\section{(Scheme 1)}

The four 3-methoxy- and 3-benzyloxy-estra-1,3,5(10)-trien-17-ol isomers (5a-8a and 9a12a) are suitable starting materials to prepare 16-triazolyl-methyl derivatives. Triazoles are 
attractive units because of their stability against metabolic degradation and their ability to form hydrogen bonds. The $\mathrm{Cu}(\mathrm{I})$-catalysed azide-alkyne cycloaddition (CuAAC) is a facile method of wide applicability for the introduction of a triazole moiety into natural products [9]. In these compounds the triazole heterocycles and their substituted derivatives are connected through a methylene linker to the sterane skeleton. The 16-p-tolylsulfonyloxymethyl ester $[5,6]$ and 16bromomethyl derivatives [10] of the 16-hydroxymethyl starting materials were used for substitution reaction with $\mathrm{NaN}_{3}$ in $N, N$-dimethylformamide to have the desired 3-methoxy-and 3-benzyloxy-16-azidomethylestra-1,3,5(10)-trien-17-ols (13-16 and 17-20). From these azido compounds several D-ring-substituted estrane derivatives containing a 1,2,3-triazole ring were synthesized by the reaction of 13-16 and 17-20 with various terminal alkynes through the use of the "click" chemistry approach to deliver compounds $21 \mathbf{a}-\mathbf{e}, 22 \mathbf{a}-\mathbf{e}, 23 \mathbf{a}-\mathbf{e}, 24 \mathbf{a}-\mathbf{e}, 25 \mathbf{a}-\mathbf{e}, \mathbf{2 6} \mathbf{a}-\mathbf{e}$, 27a-e and 28a-e.

\section{Experimental}

\subsection{General}

Melting points (Mp) were determined on a Kofler block and are uncorrected. Specific rotations were measured in $\mathrm{CHCl}_{3}$ (c 1) at $20{ }^{\circ} \mathrm{C}$ with a POLAMAT-A (Zeiss-Jena) polarimeter and are

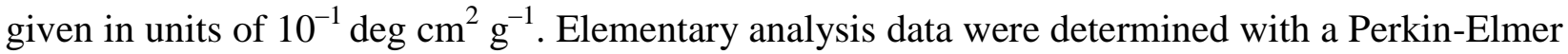
CHN analyzer model 2400. The reactions were monitored by TLC on Kieselgel-G (Merck Si 254 F) layers (0.25 $\mathrm{mm}$ thick); solvent systems (ss): (A) diisopropyl ether, (B) acetone/toluene/hexane (30:35:35 v/v). The spots were detected by spraying with 5\% phosphomolybdic acid in $50 \%$ aqueous phosphoric acid. The $R_{\mathrm{f}}$ values were determined for the spots observed by illumination at 254 and $365 \mathrm{~nm}$. Flash chromatography: silica gel 60, 40-63 $\mu \mathrm{m}$. All solvents were distilled prior to use. NMR spectra were recorded on a Bruker DRX 500 and Bruker Ascend 500 instrument at $500\left({ }^{1} \mathrm{H}\right.$ NMR) or $125 \mathrm{MHz}\left({ }^{13} \mathrm{C} \mathrm{NMR}\right)$. Chemical shifts are reported in ppm ( $\delta$ scale) and coupling constants $(J)$ in Hertz. For the determination of multiplicities, the $J$-MOD pulse sequence was used.

\subsection{3-Methoxy- and 3-benzyloxy-16-azidomethylestra-1,3,5(10)-trienes (13-16 and 17-20) General procedure}


Compounds 5b-8b [5,6] (4.70 g, $10 \mathrm{mmol})$ or 9c-12c [] (4.55 g, $10 \mathrm{mmol})$ were dissolved in $N$, $N$-dimethylformamide $(100 \mathrm{ml})$ and then $\mathrm{NaN}_{3}(2.6 \mathrm{~g})$ was added. The mixture was stirred for $12 \mathrm{~h}$ at $80{ }^{\circ} \mathrm{C}$, then poured into water $(500 \mathrm{ml})$. The precipitate separating out was filtered off and subjected to chromatographic separation with $\mathrm{CH}_{2} \mathrm{Cl}_{2} /$ hexane in different ratios.

\subsubsection{3-Methoxy-16 $\beta$-azidomethyl-estra-1,3,5(10)-trien-17 $\beta$-ol (13)}

Compound $\mathbf{5 b}$ (470 $\mathrm{mg}, 1 \mathrm{mmol}$ ) was used for the synthesis as described in Section 2.2. The crude product was chromatographed on silica gel with $\mathrm{CH}_{2} \mathrm{Cl}_{2} /$ hexane $(1: 3 \mathrm{v} / \mathrm{v})$ to yield pure 13 (318 mg, 93\%). Mp $134-135{ }^{\circ} \mathrm{C} ; R_{\mathrm{f}}=0.65$ (ss A); $[\alpha]_{\mathrm{D}}{ }^{20}=+80\left(c 1\right.$ in $\mathrm{CHCl}_{3}$ ). (Found C, 70.23; $\mathrm{H}, 8.05 . \mathrm{C}_{20} \mathrm{H}_{27} \mathrm{~N}_{3} \mathrm{O}_{2}(341.45)$ requires $\left.\mathrm{C}, 70.35 ; \mathrm{H}, 7.97 \%\right) .{ }^{1} \mathrm{H} \mathrm{NMR}\left(\delta, \mathrm{ppm}, \mathrm{CDCl}_{3}\right)$ : $0.82(\mathrm{~s}$, $\left.3 \mathrm{H}, 18-\mathrm{H}_{3}\right), 2.87\left(\mathrm{~m}, 2 \mathrm{H}, 6-\mathrm{H}_{2}\right), 3.32\left(\mathrm{dd}, 1 \mathrm{H}, J=12.5 \mathrm{~Hz}, J=7.5 \mathrm{~Hz}, 16 \mathrm{a}-\mathrm{H}_{2}\right), 3.61(\mathrm{dd}, 1 \mathrm{H}, J=$ $\left.12.5 \mathrm{~Hz}, J=7.5 \mathrm{~Hz}, 16 \mathrm{a}-\mathrm{H}_{2}\right), 3.78\left(\mathrm{~s}, 3 \mathrm{H}, 3-\mathrm{OCH}_{3}\right), 3.87$ (d, 1H, J = $\left.10.0 \mathrm{~Hz}, 17-\mathrm{H}\right), 6.64(\mathrm{~d}, 1 \mathrm{H}$, $J=2.5 \mathrm{~Hz}, 4-\mathrm{H}), 6.72(\mathrm{dd}, 1 \mathrm{H}, J=8.5 \mathrm{~Hz}, J=2.5 \mathrm{~Hz}, 2-\mathrm{H}), 7.20(\mathrm{~d}, 1 \mathrm{H}, J=8.5 \mathrm{~Hz}, 1-\mathrm{H}) .{ }^{13} \mathrm{C}$ $\operatorname{NMR}\left(\delta, \mathrm{ppm}, \mathrm{CDCl}_{3}\right): 12.2$ (C-18), 26.3, 27.5, 29.7, 30.4, 37.7, 38.2, 40.2, 44.0, 44.3 (C-13), 49.0, $53.4(\mathrm{C}-16 \mathrm{a}), 55.2\left(3-\mathrm{OCH}_{3}\right), 81.5(\mathrm{C}-17), 111.6(\mathrm{C}-2), 113.9(\mathrm{C}-4), 126.2$ (C-1), $132.5(\mathrm{C}-$ 10), 137.9 (C-5), 157.7 (C-3).

\subsubsection{3-Methoxy-16 $\alpha$-azidomethylestra-1,3,5(10)-trien-17 $\beta$-ol (14)}

Compound $6 \mathbf{b}$ (470 mg, $1 \mathrm{mmol}$ ) was used for the synthesis as described in Section 2.2. The crude product was chromatographed on silica gel with $\mathrm{CH}_{2} \mathrm{Cl}_{2} /$ hexane $(1: 3 \mathrm{v} / \mathrm{v})$ to yield pure 14 (287 mg, 84\%). $\mathrm{Mp} 85-86{ }^{\circ} \mathrm{C} ; R_{\mathrm{f}}=0.62(\mathrm{ss} \mathrm{A}) ;[\alpha]_{\mathrm{D}}{ }^{20}=+48\left(c 1\right.$ in $\left.\mathrm{CHCl}_{3}\right)$. (Found C, 70.42; $\mathrm{H}$, 7.65. $\mathrm{C}_{20} \mathrm{H}_{27} \mathrm{~N}_{3} \mathrm{O}_{2}(341.45)$ requires $\left.\mathrm{C}, 70.35 ; \mathrm{H}, 7.97 \%\right)$. ${ }^{1} \mathrm{H} \mathrm{NMR}\left(\delta, \mathrm{ppm}, \mathrm{CDCl}_{3}\right): 0.84$ (s, $3 \mathrm{H}$, 18- $\left.\mathrm{H}_{3}\right), 2.86\left(\mathrm{~m}, 2 \mathrm{H}, 6-\mathrm{H}_{2}\right), 3.43$ (d, 1H, J=7.5 Hz, 17-H), 3.48 (dd, 2H, J = 6.5 Hz, J = 3.5 Hz, $\left.16 \mathrm{a}-\mathrm{H}_{2}\right), 3.78\left(\mathrm{~s}, 3 \mathrm{H}, 3-\mathrm{OCH}_{3}\right), 6.63(\mathrm{~s}, 1 \mathrm{H}, 4-\mathrm{H}), 6.72(\mathrm{dd}, 1 \mathrm{H}, J=6.5 \mathrm{~Hz}, J=2.0 \mathrm{~Hz}, 2-\mathrm{H}), 7.20$ $(\mathrm{d}, 1 \mathrm{H}, J=8.5 \mathrm{~Hz}, 1-\mathrm{H}) .{ }^{13} \mathrm{C} \mathrm{NMR}\left(\delta, \mathrm{ppm}, \mathrm{CDCl}_{3}\right): 11.8(\mathrm{C}-18), 26.1,27.2,28.0,29.7,36.6$, 38.5, 43.6, 43.9, 44.2 (C-13), 48.5, $55.2\left(3-\mathrm{OCH}_{3}\right), 55.6$ (C-16a), 85.1 (C-17), 111.5 (C-2), 113.8 (C-4), 126.3 (C-1), 132.4 (C-10), 137.8 (C-5), 157.5 (C-3).

\subsubsection{3-Methoxy-16 $\beta$-azidomethylestra-1,3,5(10)-trien-17 $\alpha$-ol (15)}

Compound 7b (470 mg, $1 \mathrm{mmol}$ ) were used for the synthesis as described in Section 2.2. The 
crude porduct was chromatographed on silica gel with $\mathrm{CH}_{2} \mathrm{Cl}_{2} /$ hexane $(1: 3 \mathrm{v} / \mathrm{v})$ to yield pure 15 (275 mg, 80\%). Mp 96-98; ${ }^{\circ} \mathrm{C} ; R_{\mathrm{f}}=0.60$ (ss A); $[\alpha]_{\mathrm{D}}{ }^{20}=+68\left(c 1\right.$ in $\mathrm{CHCl}_{3}$ ). (Found C, 70.26; $\mathrm{H}$, 8.15. $\mathrm{C}_{20} \mathrm{H}_{27} \mathrm{~N}_{3} \mathrm{O}_{2}$ (341.45) requires $\left.\mathrm{C}, 70.35 ; \mathrm{H}, 7.97 \%\right) .{ }^{1} \mathrm{H}$ NMR ( $\delta, \mathrm{ppm}, \mathrm{CDCl}_{3}$ ): $0.76(\mathrm{~s}$, $\left.3 \mathrm{H}, 18-\mathrm{H}_{3}\right), 2.86\left(\mathrm{~m}, 2 \mathrm{H}, 6-\mathrm{H}_{2}\right), 3.43\left(\mathrm{dd}, 2 \mathrm{H}, J=7.5 \mathrm{~Hz}, \mathrm{~J}=3.0 \mathrm{~Hz}, 16 \mathrm{a}-\mathrm{H}_{2}\right), 3.61(\mathrm{~s}, 1 \mathrm{H}, 17-\mathrm{H})$, $3.78\left(\mathrm{~s}, 3 \mathrm{H}, 3-\mathrm{OCH}_{3}\right), 6.64(\mathrm{~d}, 1 \mathrm{H}, J=2.5 \mathrm{~Hz}, 4-\mathrm{H}), 6.72(\mathrm{dd}, 1 \mathrm{H}, J=8.5 \mathrm{~Hz}, J=2.5 \mathrm{~Hz}, 2-\mathrm{H})$, $7.22(\mathrm{~d}, 1 \mathrm{H}, J=8.5 \mathrm{~Hz}, 1-\mathrm{H}) .{ }^{13} \mathrm{C} \mathrm{NMR}\left(\delta, \mathrm{ppm}, \mathrm{CDCl}_{3}\right): 17.7(\mathrm{C}-18), 25.9,27.9,29.8,30.3$, 31.9, 38.6, 43.3, 45.0 (C-13), 48.9, 55.2 (3- $\left.\mathrm{OCH}_{3}\right), 55.6$ (C-16a), 83.0 (C-17), 111.5 (C-2), 113.8 (C-4), 126.3 (C-1), 132.4 (C-10), 137.9 (C-5), 157.5 (C-3).

\subsubsection{3-Methoxy-16 $\alpha$-azidomethylestra-1,3,5(10)-trien-17 $\alpha$-ol (16)}

Compound $8 \mathbf{b}$ (470 mg, $1 \mathrm{mmol}$ ) was used for the synthesis as described in Section 2.2. The crude product was chromatographed on silica gel with $\mathrm{CH}_{2} \mathrm{Cl}_{2} /$ hexane $(1: 3 \mathrm{v} / \mathrm{v})$ to yield pure $\mathbf{1 6}$ (283 mg, 86\%). Mp $118-120{ }^{\circ} \mathrm{C} ; R_{\mathrm{f}}=0.65(\mathrm{ss} \mathrm{A}) ;[\alpha]_{\mathrm{D}}{ }^{20}=+34\left(c 1\right.$ in $\left.\mathrm{CHCl}_{3}\right)$. (Found $\mathrm{C}, 70.55$; $\mathrm{H}$, 7.78. $\mathrm{C}_{20} \mathrm{H}_{27} \mathrm{~N}_{3} \mathrm{O}_{2}$ (341.45) requires $\left.\mathrm{C}, 70.35 ; \mathrm{H}, 7.97 \%\right) .{ }^{1} \mathrm{H}$ NMR ( $\delta, \mathrm{ppm}, \mathrm{CDCl}_{3}$ ): 0.80 (s, $\left.3 \mathrm{H}, 18-\mathrm{H}_{3}\right), 2.87\left(\mathrm{~m}, 2 \mathrm{H}, 6-\mathrm{H}_{2}\right), 3.35\left(\mathrm{dd}, 1 \mathrm{H}, J=12.0 \mathrm{~Hz}, J=6.0 \mathrm{~Hz}, 16 \mathrm{a}-\mathrm{H}_{2}\right), 3.53(\mathrm{dd}, 1 \mathrm{H}, J=$ $\left.12.0 \mathrm{~Hz}, J=9.5 \mathrm{~Hz}, 16 \mathrm{a}-\mathrm{H}_{2}\right), 3.78\left(\mathrm{~s}, 3 \mathrm{H}, 3-\mathrm{OCH}_{3}\right), 3.84(\mathrm{~d}, 1 \mathrm{H}, J=6.0 \mathrm{~Hz}, 17-\mathrm{H}), 6.63(\mathrm{~d}, 1 \mathrm{H}, J$ $=2.5 \mathrm{~Hz}, 4-\mathrm{H}), 6.72(\mathrm{dd}, 1 \mathrm{H}, J=8.5 \mathrm{~Hz}, 2-\mathrm{H}), 7.21(\mathrm{~d}, 1 \mathrm{H}, J=8.5 \mathrm{~Hz}, 1-\mathrm{H}) .{ }^{13} \mathrm{C}$ NMR $(\delta, \mathrm{ppm}$, $\mathrm{CDCl}_{3}$ ): 17.3 (C-18), 26.1, 28.0, 29.2, 31.3, 39.1, 40.5, 43.6, 46.4 (C-13), 47.0, 52.4 (C-16a), $55.2\left(3-\mathrm{OCH}_{3}\right), 79.9$ (C-17), 111.6 (C-2), 114.0 (C-4), 126.3 (C-1), 132.7 (C-10), 137.9 (C-5), $157.6(\mathrm{C}-3)$.

\subsubsection{3-Benzyloxy-16 $\beta$-azidomethylestra-1,3,5(10)-trien-17 $\beta$-ol (17)}

Compound 9c (455 mg, $1 \mathrm{mmol}$ ) was used for the synthesis as described in Section 2.2. The crude product was chromatographed on silica gel with $\mathrm{CH}_{2} \mathrm{Cl}_{2} /$ hexane $(1: 1 \mathrm{v} / \mathrm{v})$ to yield pure $\mathbf{1 7}$ (250 mg, 59\%). Mp 115-117 ${ }^{\circ} \mathrm{C} ; R_{\mathrm{f}}=0.45$ (ss A). (Found C, 74.55; H, 7.64. $\mathrm{C}_{26} \mathrm{H}_{31} \mathrm{~N}_{3} \mathrm{O}_{2}$ (417.54) requires $\mathrm{C}, 74.79 ; \mathrm{H}, 7.48 \%) .{ }^{1} \mathrm{H} \mathrm{NMR}\left(\delta, \mathrm{ppm}, \mathrm{CDCl}_{3}\right): 0.82\left(\mathrm{~s}, 3 \mathrm{H}, 18-\mathrm{H}_{3}\right), 2.86(\mathrm{~m}$, $\left.2 \mathrm{H}, 6-\mathrm{H}_{2}\right), 3.33\left(\mathrm{dd}, 1 \mathrm{H}, J=12.0 \mathrm{~Hz}, J=7.5 \mathrm{~Hz}, 16 \mathrm{a}-\mathrm{H}_{2}\right), 3.60(\mathrm{dd}, 1 \mathrm{H}, J=12.5 \mathrm{~Hz}, J=7.5 \mathrm{~Hz}$, 16a- $\left.\mathrm{H}_{2}\right), 3.87$ (d, 1H, $\left.J=9.5 \mathrm{~Hz}, 17-\mathrm{H}\right), 5.04$ (s, 2H, Bn-H $\mathrm{H}_{2}, 6.73$ (s, 1H, 4-H), 6.79 (d, 1H, $J=$ $8.0 \mathrm{~Hz}, J=2.0 \mathrm{~Hz}, 2-\mathrm{H}), 7.21(\mathrm{~d}, 1 \mathrm{H}, J=8.0 \mathrm{~Hz}, 1-\mathrm{H}), 7.32$ (t, $\left.1 \mathrm{H}, J=7.5 \mathrm{~Hz}, 4^{\prime}-\mathrm{H}\right), 7.39$ (t, 2H, $J=7.5 \mathrm{~Hz}, 3^{\prime}-\mathrm{H}$ and $\left.5^{\prime}-\mathrm{H}\right), 7.44$ (d, $2 \mathrm{H}, J=7.5 \mathrm{~Hz}, 2^{\prime}-\mathrm{H}$ and $\left.6^{\prime}-\mathrm{H}\right) .{ }^{13} \mathrm{C}$ NMR $\left(\delta, \mathrm{ppm}, \mathrm{CDCl}_{3}\right)$ : 12.2 (C-18), 26.2, 27.5, 29.7, 30.3, 37.6, 38.1, 40.1, 43.9, 44.2 (C-13), 48.8 (C-16), 53.3 (C-16a), 
$69.9\left(\mathrm{Bn}^{-\mathrm{CH}_{2}}\right), 81.5$ (C-17), 112.3 (C-2), 114.8 (C-4), 126.3 (C-1), 127.3 (C-2' and C-6'), 127.8 (C-4'), 128.5 (C-3' and C-5'), 132.7 (C-10), 137.3 (C-1'), 137.9 (C-5), 156.8 (C-3).

\subsubsection{3-Benzyloxy-16 $\alpha$-azidomethylestra-1,3,5(10)-trien-17 $\beta$-ol (18)}

Compound 10c (455 mg, $1 \mathrm{mmol}$ ) was used for the synthesis as described in Section 2.2. The crude product was chromatographed on silica gel with $\mathrm{CH}_{2} \mathrm{Cl}_{2} /$ hexane $(3: 1 \mathrm{v} / \mathrm{v})$ to yield pure 18 (254 mg, 61\%). Mp 75-77 ${ }^{\circ} \mathrm{C} ; R_{\mathrm{f}}=0.40$ (ss A). (Found C, 74.87; H, 7.32. $\mathrm{C}_{26} \mathrm{H}_{31} \mathrm{~N}_{3} \mathrm{O}_{2}$ (417.54) requires $\mathrm{C}, 74.79 ; \mathrm{H}, 7.48 \%) .{ }^{1} \mathrm{H} \mathrm{NMR}\left(\delta, \mathrm{ppm}, \mathrm{CDCl}_{3}\right)$ : 0.84 (s, 3H, 18- $\left.\mathrm{H}_{3}\right), 2.85\left(\mathrm{~m}, 2 \mathrm{H}, 6-\mathrm{H}_{2}\right)$, $3.44(\mathrm{t}, 1 \mathrm{H}, J=8.0 \mathrm{~Hz}, 17-\mathrm{H}), 3.48\left(\mathrm{~m}, 2 \mathrm{H}, 16 \mathrm{a}-\mathrm{H}_{2}\right), 5.04\left(\mathrm{~s}, 2 \mathrm{H}, \mathrm{Bn}-\mathrm{H}_{2}\right), 6.73$ (s, 1H, 4-H), 6.79 $(\mathrm{d}, 1 \mathrm{H}, \mathrm{J}=8.5 \mathrm{~Hz}, 2-\mathrm{H}), 7.21(\mathrm{~d}, 1 \mathrm{H}, J=8.5 \mathrm{~Hz}, 1-\mathrm{H}), 7.32$ (t, $\left.1 \mathrm{H}, J=7.0 \mathrm{~Hz}, 4^{\prime}-\mathrm{H}\right), 7.39$ (t, 2H, $J=7.0 \mathrm{~Hz}, 3^{\prime}$ ' - and 5'-H), $7.44\left(\mathrm{~d}, 2 \mathrm{H}, J=7.0 \mathrm{~Hz}, 2^{\prime}\right.$ '- and $\left.6^{\prime}-\mathrm{H}\right) .{ }^{13} \mathrm{C} \mathrm{NMR}\left(\delta, \mathrm{ppm}, \mathrm{CDCl}_{3}\right): 11.8$ (C-18), 26.1, 27.2, 27.9, 29.7, 36.6, 38.5, 43.6, 43.9, 44.2 (C-13), 48.6 (C-16), 55.6 (C-16a), 69.9 (Bn-CH ${ }_{2}$ ), 85.1 (C-17), 112.3 (C-2), 114.8 (C-4), 126.3 (C-1), 127.4 (C-2' and -6'), 127.8 (C-4'), 128.5 (C-3' and -5'), 132.7 (C-10), 137.3 (C-1'), 137.9 (C-5), 156.8 (C-3).

\subsubsection{3-Benzyloxy-16 $\beta$-azidomethyl-estra-1,3,5(10)-trien-17 $\alpha$-ol (19)}

Copound 11c (455 mg, $1 \mathrm{mmol}$ ) was used for the synthesis as described in Section 2.2. The crude product was chromatographed on silica gel with $\mathrm{CH}_{2} \mathrm{Cl}_{2} /$ hexane (3:1 v/v) to yield pure 19 (23. mg, $40 \%$ ). Mp. $134-136{ }^{\circ} \mathrm{C} . R_{\mathrm{f}}=0.38$ (ss A). (Found C, 74.92; H, 7.37. $\mathrm{C}_{26} \mathrm{H}_{31} \mathrm{~N}_{3} \mathrm{O}_{2}$ (417.54) requires $\mathrm{C}, 74.79 ; \mathrm{H}, 7.48 \%) .{ }^{1} \mathrm{H} \mathrm{NMR}\left(\delta, \mathrm{ppm}, \mathrm{CDCl}_{3}\right): 0.84$ (s, 3H, 18- $\left.\mathrm{H}_{3}\right), 2.85$ (m, 2H, 6- $\mathrm{H}_{2}$ ), $3.43(\mathrm{~d}, 2 \mathrm{H}, J=8.0 \mathrm{~Hz}, 17-\mathrm{H}), 3.48\left(\mathrm{t}, 2 \mathrm{H}, \mathrm{J}=6.5 \mathrm{~Hz}, 16 \mathrm{a}-\mathrm{H}_{2}\right), 5.04\left(\mathrm{~s}, 2 \mathrm{H}, \mathrm{Bn}-\mathrm{H}_{2}\right), 6.73(\mathrm{~s}, 1 \mathrm{H}$, 4-H), 6.79 (d, 1H, $J=8.0 \mathrm{~Hz}, 2-\mathrm{H}), 7.22$ (d, 1H, $J=8.0 \mathrm{~Hz} 1-\mathrm{H}), 7.33$ (d, 1H, $J=7.0 \mathrm{~Hz}, 4^{\prime}-\mathrm{H}$ ), 7.39 (t, $2 \mathrm{H}, J=7.0 \mathrm{~Hz}, 3^{\prime}$ ' and $\left.5^{\prime}-\mathrm{H}\right), 7.44\left(\mathrm{~d}, 2 \mathrm{H}, J=7.0 \mathrm{~Hz}, 2^{\prime}-\right.$ and $\left.6^{\prime}-\mathrm{H}\right) .{ }^{13} \mathrm{C}$ NMR ( $\delta$, ppm, $\left.\mathrm{CDCl}_{3}\right): 11.8$ (C-18), 26.1, 27.2, 28.0, 29.7, 36.6, 38.4, 43.5, 43.9, 44.1 (C-13), 48.5 (C-16), 55.6 (C-16a), 69.9 (Bn-CH $\left.{ }_{2}\right), 85.1$ (C-17), 112.3 (C-2), 114.8 (C-4), 126.3 (C-1), 127.4 (C-2' and -6'), 127.8 (C-4'), 128.5 (C-3' and -5'), 132.7 (C-10), 137.3 (C-1'), 137.9 (C-5), 156.7 (C-3).

\subsubsection{3-Benzyloxy-16 $\alpha$-azidomethyl-estra-1,3,5(10)-trien-17 $\alpha$-ol (20)}

Compound 12c (455 mg, $1 \mathrm{mmol}$ ) was used for the synthesis as described in Section 2.2. The crude was chromatographed on silica gel with $\mathrm{CH}_{2} \mathrm{Cl}_{2} /$ hexane $(1: 1 \mathrm{v} / \mathrm{v})$ to yield pure $2 \mathbf{0}$ (330 mg, 79\%). Mp 90-92 ${ }^{\circ}$ C. $R_{\mathrm{f}}=0.45$ (ss A). (Found C, 74.68; H, 7.55. $\mathrm{C}_{26} \mathrm{H}_{31} \mathrm{~N}_{3} \mathrm{O}_{2}$ (417.54) requires C, 
74.79; H, 7.48\%). ${ }^{1} \mathrm{H}$ NMR ( $\delta$, ppm, $\left.\mathrm{CDCl}_{3}\right): 0.79\left(\mathrm{~s}, 3 \mathrm{H}, 18-\mathrm{H}_{3}\right), 2.71\left(\mathrm{~m}, 2 \mathrm{H}, 6-\mathrm{H}_{2}\right), 3.35(\mathrm{dd}$, $\left.1 \mathrm{H}, J=12.0 \mathrm{~Hz}, J=6.5 \mathrm{~Hz}, 16 \mathrm{a}-\mathrm{H}_{2}\right), 3.52\left(\mathrm{dd}, 1 \mathrm{H}, J=12.0 \mathrm{~Hz}, J=6.5 \mathrm{~Hz}, 16 \mathrm{a}-\mathrm{H}_{2}\right), 3.84(\mathrm{~d}, 1 \mathrm{H}$, $J=5.0 \mathrm{~Hz}, 17-\mathrm{H}), 5.04\left(\mathrm{~s}, 2 \mathrm{H}, \mathrm{Bn}-\mathrm{H}_{2}\right), 6.73(\mathrm{~s}, 1 \mathrm{H}, 4-\mathrm{H}), 6.79(\mathrm{dd}, 1 \mathrm{H}, J=8.5 \mathrm{~Hz}, J=2.5 \mathrm{~Hz}, 2-$ H), $7.22(\mathrm{~d}, 1 \mathrm{H}, J=8.5 \mathrm{~Hz}, 1-\mathrm{H}), 7.33$ (t, $\left.1 \mathrm{H}, J=7.5 \mathrm{~Hz}, 4^{\prime}-\mathrm{H}\right), 7.39$ (t, $2 \mathrm{H}, J=7.5 \mathrm{~Hz}, 3^{\prime}-$ and $\left.5^{\prime}-\mathrm{H}\right), 7.44\left(\mathrm{~d}, 2 \mathrm{H}, J=7.5 \mathrm{~Hz}, 2^{\prime}-\right.$ and $\left.6{ }^{\prime}-\mathrm{H}\right) .{ }^{13} \mathrm{C}$ NMR ( $\left.\delta, \mathrm{ppm}, \mathrm{CDCl}_{3}\right)$ : $17.2(\mathrm{C}-18), 26.0,27.9$,

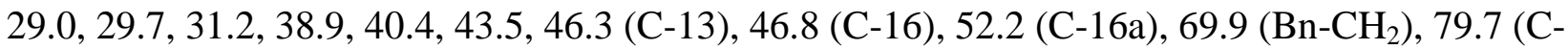
17), 112.3 (C-2), 114.8 (C-4), 126.3 (C-1), 127.4 (C-2' and -6'), 127.8 (C-4'), 128.5 (C-3' and 5'), 132.8 (C-10), 137.3 (C-1'), 138.0 (C-5), 156.7 (C-3).

2.3. General procedure for the synthesis of triazoles (21a-e, 22a-e, 23a-e, 24a-e, 25a-e, 26a-e, $27 \boldsymbol{a}-\boldsymbol{e}$, and $28 \boldsymbol{a}-\boldsymbol{e}$ )

3-Methoxy-16-azidomethylestra-1,3,5(10)-trien-17-ol isomers (13-16) $(342 \mathrm{mg}, 1 \mathrm{mmol})$ or 3benzyloxy-16-azidomethylestra-1,3,5(10)-trien-17-ol isomers (17-20) $418 \mathrm{mg}, 1 \mathrm{mmol}$ ) were dissolved in $\mathrm{CH}_{2} \mathrm{Cl}_{2}(20 \mathrm{ml})$, then $\mathrm{CuI}(19 \mathrm{mg}, 0.10 \mathrm{mmol}), \mathrm{Et}_{3} \mathrm{~N}(0.2 \mathrm{ml}, 2 \mathrm{mmol})$ and the appropriate terminal alkynes $(2 \mathrm{mmol})$ were added. The mixtures were stirred under reflux for 24 $\mathrm{h}$, then diluted with water $(30 \mathrm{ml})$ and extracted with $\mathrm{CH}_{2} \mathrm{Cl}_{2}(2 \times 30 \mathrm{ml})$. The combined organic phases were dried over $\mathrm{Na}_{2} \mathrm{SO}_{4}$ and evaporated in vacuo. The crude products were purified by flash chromatography using $\mathrm{CH}_{2} \mathrm{Cl}_{2} /$ ethyl acetate in different ratios.

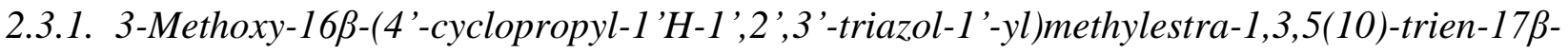 ol (21a)}

Compound 13 (342 mg, $1 \mathrm{mmol}$ ) and cyclopropylacetylene (2 mmol, $0.22 \mathrm{ml})$ were used for the synthesis as described in Section 2.3. The crude product was chromatographed on silica gel with $\mathrm{CH}_{2} \mathrm{Cl}_{2} /$ hexane $(3: 1 \mathrm{v} / \mathrm{v})$ to yield pure $21 \mathrm{a}(210 \mathrm{mg}, 51 \%)$ as a white solid. Mp: $189-191{ }^{\circ} \mathrm{C} ; R_{\mathrm{f}}=$ 0.44 (ss B). (Found C, 73.84; H, 7.98. $\mathrm{C}_{25} \mathrm{H}_{33} \mathrm{~N}_{3} \mathrm{O}_{2}$ (407.55) requires C, 73.68; H, 8.16\%). ${ }^{1} \mathrm{H}$ NMR $\left(\delta, \mathrm{ppm}, \mathrm{CDCl}_{3}\right): 0.80\left(\mathrm{~s}, 3 \mathrm{H}, 18-\mathrm{H}_{3}\right), 0.83\left(\mathrm{~s}, 2 \mathrm{H}\right.$, cyclopropyl- $\left.\mathrm{H}_{2}\right), 0.94(\mathrm{~s}, 2 \mathrm{H}$, cyclopropyl- $\left.\mathrm{H}_{2}\right), 2.72\left(\mathrm{~d}, 1 \mathrm{H}, J=7.0 \mathrm{~Hz}, 1\right.$ '-H), $2.84\left(\mathrm{~m}, 2 \mathrm{H}, 6-\mathrm{H}_{2}\right), 3.77\left(\mathrm{~s}, 3 \mathrm{H}, 3-\mathrm{OCH}_{3}\right), 3.93$ $(\mathrm{d}, 1 \mathrm{H}, J=9.5 \mathrm{~Hz}, 17-\mathrm{H}), 4.21\left(\mathrm{dd}, 1 \mathrm{H}, J=13.0 \mathrm{~Hz}, J=6.0 \mathrm{~Hz}, 16 \mathrm{a}-\mathrm{H}_{2}\right), 4.62(\mathrm{t}, 1 \mathrm{H}, J=8.0 \mathrm{~Hz}$, 16a- $\mathrm{H}_{2}$ ), 6.62 (s, 1H, 4-H), 6.71 (d, 1H, $\left.J=8.5 \mathrm{~Hz}, 2-\mathrm{H}\right), 7.20$ (d, 1H, $\left.J=8.5 \mathrm{~Hz}, 1-\mathrm{H}\right), 7.29$ (s, $\left.1 \mathrm{H}, 5^{\prime}-\mathrm{H}\right) .{ }^{13} \mathrm{C}$ NMR $\left(\delta, \mathrm{ppm}, \mathrm{CDCl}_{3}\right): 6.7$ (C-1"), 7.68 (C-2” and -3"),12.3 (C-18), 26.2, 27.4, 
29.7, 30.8, 37.5, 38.0, 41.4, 43.8, 44.3 (C-16a), 48.7, 51.7 (C-13), $55.2\left(3-\mathrm{OCH}_{3}\right), 80.7(\mathrm{C}-17)$, 111.5 (C-2), 113.8 (C-4), 126.3 (C-1), 132.4 (C-10), 137.8 (C-5), 157.5 (C-3).

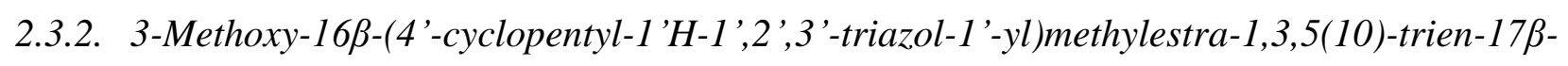
ol $(21 b)$

Compound 13 (342 mg, $1 \mathrm{mmol})$ and cyclopentylacetylene $(2 \mathrm{mmol}, 0.22 \mathrm{ml})$ were used for the synthesis as described in Section 2.3. The crude product was chromatographed on silica gel with $\mathrm{CH}_{2} \mathrm{Cl}_{2}$ to yield pure 21b (370 mg, $85 \%$ ) as a white solid. Mp: $191-192{ }^{\circ} \mathrm{C} ; R_{\mathrm{f}}=0.46$ (ss B). (Found C, 74.62; $\mathrm{H}, 8.42 . \mathrm{C}_{27} \mathrm{H}_{37} \mathrm{~N}_{3} \mathrm{O}_{2}$ (435.60) requires $\left.\mathrm{C}, 74.45 ; \mathrm{H}, 8.56 \%\right) .{ }^{1} \mathrm{H}$ NMR $(\delta$, ppm,

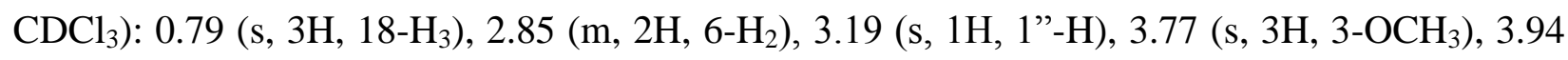
$(\mathrm{d}, 1 \mathrm{H}, J=9.5 \mathrm{~Hz}, 17-\mathrm{H}), 4.24\left(\mathrm{~d}, 1 \mathrm{H}, J=8.0 \mathrm{~Hz}, 16 \mathrm{a}-\mathrm{H}_{2}\right), 4.65\left(\mathrm{~s}, 1 \mathrm{H}, 16 \mathrm{a}-\mathrm{H}_{2}\right), 6.62(\mathrm{~s}, 1 \mathrm{H}, 4-$ H), $6.71(\mathrm{~d}, 1 \mathrm{H}, J=8.5 \mathrm{~Hz}, 2-\mathrm{H}), 7.20(\mathrm{~d}, 1 \mathrm{H}, J=8.5 \mathrm{~Hz}, 1-\mathrm{H}), 7.34$ (s, $\left.1 \mathrm{H}, 5^{\prime}-\mathrm{H}\right) .{ }^{13} \mathrm{C}$ NMR $(\delta$, ppm, $\mathrm{CDCl}_{3}$ ): 12.3 (C-18), 25.1 (C-3" and -4"), 26.2, 27.4, 29.7 (C-2" and 5"), 30.8, 33.2, 36.7, 37.5, 38.0, 42.4 (C-16a), 43.8, 44.3 (C-13), 48.7, 51.8, 55.2 (3-OCH $), 62.1$ (C-16), 80.7 (C-17), 111.5 (C-2), 113.7 (C-4), 126.3 (C-1), 132.4 (C-10), 137.8 (C-5), 157.4 (C-3).

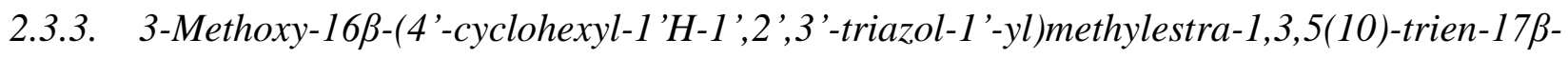 ol (21c)}

Compound 13 (342 mg, $1 \mathrm{mmol})$ and cyclohexylacetylene $(2 \mathrm{mmol}, 0.22 \mathrm{ml})$ were used for the synthesis as described in Section 2.3. The crude product was chromatographed on silica gel with ethyl acetate/ $\mathrm{CH}_{2} \mathrm{Cl}_{2}(1: 99 \mathrm{v} / \mathrm{v})$ to yield pure $21 \mathrm{c}(370 \mathrm{mg}, 82 \%)$ as a white solid. Mp: 189-190 ${ }^{\circ} \mathrm{C} ; R_{\mathrm{f}}=0,40$ (ss B). (Found C, 74.92; H, 8.55. $\mathrm{C}_{28} \mathrm{H}_{41} \mathrm{~N}_{3} \mathrm{O}_{2}(449.63$ ) requires $\mathrm{C}, 74.80 ; \mathrm{H}, 8.74 \%$ ). ${ }^{1} \mathrm{H}$ NMR ( $\delta$, ppm, $\mathrm{CDCl}_{3}$ ): 0.79 (s, 3H, 18- $\mathrm{H}_{3}$ ), 2.84 (m, 2H, 6- $\mathrm{H}_{2}$ ), 3.77 (s, 3H, 3- $\mathrm{OCH}_{3}$ ), 3.94 $(\mathrm{d}, 1 \mathrm{H}, J=9.5 \mathrm{~Hz}, 17-\mathrm{H}), 4.24\left(\mathrm{~m}, 1 \mathrm{H}, 16 \mathrm{a}-\mathrm{H}_{2}\right), 4.65\left(\mathrm{~m}, 1 \mathrm{H}, 16 \mathrm{a}-\mathrm{H}_{2}\right), 6.62(\mathrm{~s}, 1 \mathrm{H}, 4-\mathrm{H}), 6.71(\mathrm{~d}$, $1 \mathrm{H}, J=8.5 \mathrm{~Hz}, 2-\mathrm{H}), 7.20(\mathrm{~d}, 1 \mathrm{H}, J=8.5 \mathrm{~Hz}, 1-\mathrm{H}), 7.32\left(\mathrm{~s}, 1 \mathrm{H}, 5^{\prime}-\mathrm{H}\right) .{ }^{13} \mathrm{C}$ NMR $(\delta, \mathrm{ppm}$, $\mathrm{CDCl}_{3}$ ): 12.3 (C-18), 26.0, 26.1 (C-2" and -6"), 26.2, 27.4, 29.7, 30.8, 33.0, 37.5, 38.0, 41.4 (C1”), 43.8, 44.3 (C-13), 48.3, $55.2\left(3-\mathrm{OCH}_{3}\right), 62.1,80.7$ (C-17), 111.5 (C-2), 113.7 (C-4), 126.3 (C-1), 132.4 (C-10), 137.8 (C-5), 157.4 (C-3).

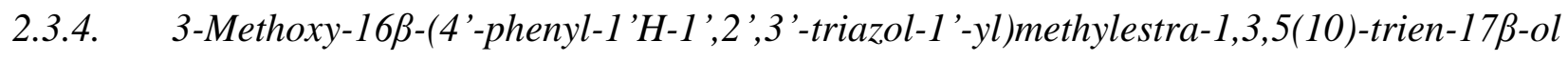
(21d) 
Compound $13(342 \mathrm{mg}, 1 \mathrm{mmol})$ and phenylacetylene $(2 \mathrm{mmol}, 0.22 \mathrm{ml})$ were used for the synthesis as described in Section 2.3. The crude product was chromatographed on silica gel with ethyl acetate $/ \mathrm{CH}_{2} \mathrm{Cl}_{2}(1: 99 \mathrm{v} / \mathrm{v})$ to yield pure 21d (368 mg, 83\%) as a white solid. Mp: 232-234 ${ }^{\circ} \mathrm{C} ; R_{\mathrm{f}}=0.35$ (ss B). (Found C, 75.98; H, 7.36. $\mathrm{C}_{28} \mathrm{H}_{33} \mathrm{~N}_{3} \mathrm{O}_{2}$ (443.58) requires $\mathrm{C}, 75.81 ; \mathrm{H}, 7.50 \%$ ). ${ }^{1} \mathrm{H}$ NMR ( $\delta$, ppm, $\mathrm{CDCl}_{3}$ ): 0.79 (s, 3H, 18- $\mathrm{H}_{3}$ ), 2.73 (m, 2H, 6- $\left.\mathrm{H}_{2}\right), 3.68\left(\mathrm{~s}, 3 \mathrm{H}, 3-\mathrm{OCH}_{3}\right), 3.79$ $(\mathrm{d}, 1 \mathrm{H}, J=10.0 \mathrm{~Hz}, 17-\mathrm{H}), 4.20\left(\mathrm{t}, 1 \mathrm{H}, J=13.5 \mathrm{~Hz}, 16 \mathrm{a}-\mathrm{H}_{2}\right), 4.63(\mathrm{dd}, 1 \mathrm{H}, J=13.5 \mathrm{~Hz}, J=4.5$ Hz, 16a- $\left.\mathrm{H}_{2}\right), 6.59$ (s, 1H, 4-H), 6.67 (d, 1H, $\left.J=8.5 \mathrm{~Hz}, 2-\mathrm{H}\right), 7.16$ (d, 1H, $\left.J=8.5 \mathrm{~Hz}, 1-\mathrm{H}\right), 7.32$ (t, $1 \mathrm{H}, J=7.5 \mathrm{~Hz}, 4$ "'-H), 7.44 (t, 2H, $J=7.5 \mathrm{~Hz}, 3$ "'- and 5"-H), 7.85 (d, 2H, $J=7.5 \mathrm{~Hz}, 2$ "'- and 6"-H), 8.60 (s, $1 \mathrm{H}, 5$ '-H). ${ }^{13} \mathrm{C}$ NMR ( $\delta$, ppm, $\mathrm{CDCl}_{3}$ ): 12.4 (C-18), 25.8, 26.9, 29.1, 30.0, 36.9, 37.8, 40.4, 43.3, 43.7 (C-13), 47.8, 52.3 (C-16a), 54.8 (3-- $\left.\mathrm{OCH}_{3}\right), 79.5$ (C-17), 111.4 (C-2), 113.3 (C-4), 121.5 (C-5'), 124.5 (C-2" and -6”), 126.0 (C-1), 127.6 (C-4”), 127.8 (C-3" and -5"), 130.9 (C-1”), 132.0 (C-10), 137.3 (C-5), 146.0 (C-4'), 156.9 (C-3).

2.3.5. 3-Methoxy-16ß-(4'-nitro-benzoyloxymethyl-1 'H-1',2, '3'-triazol-1'-yl)methylestra1,3,5(10)-trien-17 $\beta$-ol (21e)

Compound 13 (342 mg, $1 \mathrm{mmol}$ ) and propargyl 4-nitrobenzoate ( $2 \mathrm{mmol}, 410 \mathrm{mg}$ ) were used for the synthesis as described in Section 2.3. The crude product was chromatographed on silica gel with ethyl acetate/ $\mathrm{CH}_{2} \mathrm{Cl}_{2}(5: 95 \mathrm{v} / \mathrm{v})$ to yield pure $21 \mathrm{e}(475 \mathrm{mg}, 86 \%)$ as a yellow solid. Mp: 134-135.5 ${ }^{\circ} \mathrm{C} ; R_{\mathrm{f}}=30$ (ss B). (Found C, 66.12; H, 6.08. $\mathrm{C}_{30} \mathrm{H}_{34} \mathrm{~N}_{4} \mathrm{O}_{6}(546.61$ ) requires C, 65.92; $\mathrm{H}, 6.27 \%) .{ }^{1} \mathrm{H}$ NMR $\left(\delta, \mathrm{ppm}, \mathrm{CDCl}_{3}\right): 0.73$ (s, 3H, 18- $\left.\mathrm{H}_{3}\right), 2.70\left(\mathrm{~m}, 2 \mathrm{H}, 6-\mathrm{H}_{2}\right), 3.66(\mathrm{~s}, 3 \mathrm{H}, 3-$ $\left.\mathrm{OCH}_{3}\right), 4.18(\mathrm{dd}, 1 \mathrm{H}, J=13.5 \mathrm{~Hz}, J=11.5 \mathrm{~Hz}, 16 \mathrm{a}-\mathrm{H} 2), 4.58(\mathrm{dd}, 1 \mathrm{H}, J=13.5 \mathrm{~Hz}, J=4.5 \mathrm{~Hz}$, $\left.16 \mathrm{a}-\mathrm{H}_{2}\right), 5.02(\mathrm{~d}, 1 \mathrm{H}, J=4.5 \mathrm{~Hz}, 17-\mathrm{H}), 5.44\left(\mathrm{~s}, 2 \mathrm{H}, 4^{\prime}-\mathrm{H}_{2}\right), 6.55$ (d, 1H, $\left.J=1.5 \mathrm{~Hz}, 4-\mathrm{H}\right), 6.63$ $(\mathrm{dd}, 1 \mathrm{H}, J=8.5 \mathrm{~Hz}, J=2.0 \mathrm{~Hz}, 2-\mathrm{H}), 7.12(\mathrm{~d}, 1 \mathrm{H}, J=8.5 \mathrm{~Hz}, 1-\mathrm{H}), 8.16$ (d, 2H, $J=8.5 \mathrm{~Hz}, 3$ ”and 5"-H), 8.31 (t, 3H, $J=8.5 \mathrm{~Hz}, 2$ "'- and 6"-H, 5 '-H). ${ }^{13} \mathrm{C}$ NMR ( $\left.\delta, \mathrm{ppm}, \mathrm{CDCl}_{3}\right)$ : $12.3(\mathrm{C}-18)$, 25.8, 26.9, 29.1, 30.0, 36.9, 37.8, 40.4, 43.3, 43.7 (C-13), 47.8, 52.2 (C-16a), $54.7\left(3-\mathrm{OCH}_{3}\right)$, 58.7 (4'- $\mathrm{CH}_{2}$ ), 79.5 (C-17), 111.3 (C-2), 113.3 (C-4), 123.8 (C-2" and -6”), 125.1 (C-5'), 126.0 (C-1), 130.6 (C-3" and -5"), 131.9 (C-10), 134.7 (C-1"), 137.2 (C-5), 141.0 (C-4"), 150.2 (C-4'), $156.9(\mathrm{C}-3), 163.9(\mathrm{C}=\mathrm{O})$.

2.3.6. 3-Methoxy-16ß-(4'-hydroxymethyl-1 'H-1 ',2',3'-triazol-1'-yl)methylestra-1,3,5(10)-trien$17 \beta-o l(21 f)$ 
Compound 13 (274 mg, $0.5 \mathrm{mmol}$ ) was dissolved in methanol (10 ml) containing $\mathrm{NaOCH}_{3}(14$ $\mathrm{mg}, 0.25 \mathrm{mmol}$ ), and the solution was allowed to stand for $24 \mathrm{~h}$. It was then diluted with water, and the precipitate separating out was filtered off and recrystallized from a mixture of ethyl acetate/hexane to afford $21 \mathrm{f}(171 \mathrm{mg}, 86 \%)$ as a white crystalline material. Mp: $194-195{ }^{\circ} \mathrm{C} ; R_{\mathrm{f}}=$ 0.25 (ss B). (Found C, 69.23; H, 8.04. $\mathrm{C}_{23} \mathrm{H}_{31} \mathrm{~N}_{3} \mathrm{O}_{3}$ (397.51) requires $\mathrm{C}, 69.49 ; \mathrm{H}, 7.86 \%$ ). ${ }^{1} \mathrm{H}$ NMR ( $\delta$, ppm, DMSO-d $\left.\mathrm{d}_{6}\right): 0.76\left(\mathrm{~s}, 3 \mathrm{H}, 18-\mathrm{H}_{3}\right), 2.71\left(\mathrm{~m}, 2 \mathrm{H}, 6-\mathrm{H}_{2}\right), 3.68\left(\mathrm{~s}, 3 \mathrm{H}, 3-\mathrm{OCH}_{3}\right), 3.76$ $(\mathrm{d}, 1 \mathrm{H}, J=5.5 \mathrm{~Hz}, 17-\mathrm{H}), 4.14\left(\mathrm{t}, 1 \mathrm{H}, J=12.5 \mathrm{~Hz}, 16 \mathrm{a}-\mathrm{H}_{2}\right), 4.49$ (m, 3H, 4'- $\mathrm{H}_{2}$ and $\left.16 \mathrm{a}-\mathrm{H}_{2}\right)$, 5.03 (d, $1 \mathrm{H}, J=3.5 \mathrm{~Hz}, 17-\mathrm{OH}), 5.15$ (brs, $\left.1 \mathrm{H}, \mathrm{CH}_{2}-\mathrm{OH}\right), 6.59$ (s, 1H, 4-H), 6.66 (d, 1H, $J=8.5$ $\mathrm{Hz}, 2-\mathrm{H}), 7.16(\mathrm{~d}, 1 \mathrm{H}, J=8.5 \mathrm{~Hz}, 1-\mathrm{H}), 7.99\left(\mathrm{~s}, 1 \mathrm{H}, 5{ }^{\prime}-\mathrm{H}\right) .{ }^{13} \mathrm{C}$ NMR $\left(\delta, \mathrm{ppm}\right.$, DMSO-d $\left.{ }_{6}\right): 12.4$ (C-18), 25.9, 26.9, 29.2, 30.0, 36.9, 37.9, 40.5, 43.4, 43.8 (C-13), 47.8, 52.0 (C-16a), 54.8 (3$\left.\mathrm{OCH}_{3}\right), 55.0$ (4'- $\left.\mathrm{CH}_{2}\right), 79.5$ (C-17), 111.4 (C-2), 113.4 (C-4), 122.8 (C-5'), 126.1 (C-1), 132.0 (C-10), 137.3 (C-5), 147.6 (C-4'), 157.0 (C-3).

2.3.7. 3-Methoxy-16a-(4'-cyclopropyl-1 'H-1',2',3'-triazol-1'-yl)methylestra-1,3,5(10)-trien-17ßol $(22 a)$

Compound 14 (342 mg, $1 \mathrm{mmol})$ and cyclopropylacetylene (2 mmol, $0.22 \mathrm{ml})$ were used for the synthesis as described in Section 2.3. The crude product was chromatographed on silica gel with ethyl acetate/ $\mathrm{CH}_{2} \mathrm{Cl}_{2}(5: 95 \mathrm{v} / \mathrm{v})$ to yield pure 22a $(261 \mathrm{mg}, 64 \%)$ as a white solid. Mp: $67-69{ }^{\circ} \mathrm{C}$; $R_{\mathrm{f}}=0.35$ (ss B). (Found C, 73.55; H, 7.98. $\mathrm{C}_{25} \mathrm{H}_{33} \mathrm{~N}_{3} \mathrm{O}_{2}$ (407.55) requires C, 73.68; H, 8.16\%). ${ }^{1} \mathrm{H}$ $\operatorname{NMR}\left(\delta, p p m, \mathrm{CDCl}_{3}\right): 0.82\left(\mathrm{~m}, 5 \mathrm{H}, 18-\mathrm{H}_{3}\right.$ and cyclopropyl- $\left.\mathrm{H}_{2}\right), 0.95\left(\mathrm{~m}, 2 \mathrm{H}\right.$, cyclopropyl- $\left.\mathrm{H}_{2}\right)$, $2.83\left(\mathrm{~m}, 2 \mathrm{H}, 6-\mathrm{H}_{2}\right), 3.53(\mathrm{~d}, 1 \mathrm{H}, J=7.5 \mathrm{~Hz}, 17-\mathrm{H}), 3.77\left(\mathrm{~s}, 3 \mathrm{H}, 3-\mathrm{OCH}_{3}\right), 4.35(\mathrm{t}, 1 \mathrm{H}, J=7.5 \mathrm{~Hz}$, $\left.16 \mathrm{a}-\mathrm{H}_{2}\right), 4.44\left(\mathrm{dd}, 1 \mathrm{H}, J=13.5 \mathrm{~Hz}, J=7.5 \mathrm{~Hz}, 16 \mathrm{a}-\mathrm{H}_{2}\right), 6.62(\mathrm{~d}, 1 \mathrm{H}, J=2.0 \mathrm{~Hz}, 4-\mathrm{H}), 6.70$ (dd, $1 \mathrm{H}, J=8.5 \mathrm{~Hz}, J=2.0 \mathrm{~Hz}, 2-\mathrm{H}), 7.18(\mathrm{~d}, 1 \mathrm{H}, J=8.5 \mathrm{~Hz}, 1-\mathrm{H}) .{ }^{13} \mathrm{C}$ NMR $\left(\delta, \mathrm{ppm}, \mathrm{CDCl}_{3}\right): 6.7$ (C-1"), 7.7 (C-2" and -3"), 11.8 (C-18), 26.1, 27.2, 28.2, 29.7, 36.6, 38.4, 43.9, 44.3, 44,3 (C16a), 48.3, 54.5 (C-13), 62.1 (3- $\left.\mathrm{OCH}_{3}\right), 85.1$ (C-17), 111.5 (C-2), 113.8 (C-4), 126.2 (C-1), 132.3 (C-10), 137.8 (C-5), 157.4 (C-3).

\subsubsection{3-Methoxy-16a-(4'-cyclopentyl-1 'H-1 ',2',3'-triazol-1-yl)methylestra-1,3,5(10)-trien-17ß- ol (22b)}

Compound 14 (342 mg, $1 \mathrm{mmol})$ and cyclopentylacetylene (2 mmol, $0.22 \mathrm{ml})$ were used for the synthesis as described in Section 2.3. The crude product was chromatographed on silica gel with 
ethyl acetate $/ \mathrm{CH}_{2} \mathrm{Cl}_{2}(5: 95 \mathrm{v} / \mathrm{v})$ to yield pure $22 \mathrm{~b}(290 \mathrm{mg}, 66 \%)$ as a white solid. Mp: $163-165$ ${ }^{\circ} \mathrm{C} ; R_{\mathrm{f}}=0.32$ (ss B). (Found C, 74.63; $\mathrm{H}, 8.41 . \mathrm{C}_{27} \mathrm{H}_{37} \mathrm{~N}_{3} \mathrm{O}_{2}$ (435.60) requires $\mathrm{C}, 74.45 ; \mathrm{H}, 8.56 \%$ ). ${ }^{1} \mathrm{H}$ NMR ( $\delta$, ppm, $\mathrm{CDCl}_{3}$ ): 0.83 (s, 3H, 18- $\left.\mathrm{H}_{3}\right), 1.68$ (s, 4H, 3"- and 4"- $\left.\mathrm{H}_{2}\right), 2.83\left(\mathrm{~m}, 2 \mathrm{H}, 6-\mathrm{H}_{2}\right)$, 3.19 (m, 1H, 1"-H), 3.56 (d, 1H, J = 7.0 Hz, 17-H), 3.77 (s, 3H, 3- $\left.-\mathrm{OCH}_{3}\right), 4.43$ (m, 2H, 16a- $\mathrm{H}_{2}$ ), $6.62(\mathrm{~s}, 1 \mathrm{H}, 4-\mathrm{H}), 6.70$ (d, 1H, $J=8.5 \mathrm{~Hz}, 2-\mathrm{H}), 7.19$ (d, 1H, $J=8.5 \mathrm{~Hz}, 1-\mathrm{H}), 7.35$ (s, 1H, 5'-H). ${ }^{13} \mathrm{C}$ NMR ( $\delta$, ppm, $\mathrm{CDCl}_{3}$ ): 11.9 (C-18), 25.1 (C-3" and -4"), 26.1, 27.2, 28.3, 29.7 (C-2" and 5”), 33.2, 36.6, 38.4, 43.9, 44.2, 44.3 (C-13), 48.4, $55.2\left(3-\mathrm{OCH}_{3}\right), 62.1$ (C-16a), 85.3 (C-17), 111.5 (C-2), 113.8 (C-4), 126.3 (C-1), 132.3 (C-10), 137.8 (C-5), 157.5 (C-3).

\subsubsection{3-Methoxy-16a-(4'-cyclohexyl-1'H-1',2',3'-triazol-1'-yl)methylestra-1,3,5(10)-trien-17ß- ol $(22 c)$}

Compound 14 (342 mg, $1 \mathrm{mmol})$ and cyclohexylacetylene $(2 \mathrm{mmol}, 0.22 \mathrm{ml})$ were used for the synthesis as described in Section 2.3. The crude product was chromatographed on silica gel with ethyl acetate $/ \mathrm{CH}_{2} \mathrm{Cl}_{2}(5: 95 \mathrm{v} / \mathrm{v})$ to yield pure $22 \mathrm{c}(345 \mathrm{mg}, 76 \%)$ as a white solid. Mp: $80-82{ }^{\circ} \mathrm{C}$; $R_{\mathrm{f}}=0.34$ (ss B). (Found 74.96; H, 8.54. $\mathrm{C}_{28} \mathrm{H}_{41} \mathrm{~N}_{3} \mathrm{O}_{2}$ (449.63) requires $\mathrm{C}, 74.80 ; \mathrm{H}, 8.74 \%$ ). ${ }^{1} \mathrm{H}$ $\operatorname{NMR}\left(\delta, p p m, \mathrm{CDCl}_{3}\right): 0.83\left(\mathrm{~s}, 3 \mathrm{H}, 18-\mathrm{H}_{3}\right), 2.83\left(\mathrm{~m}, 2 \mathrm{H}, 6-\mathrm{H}_{2}\right), 3.55(\mathrm{~s}, 1 \mathrm{H}, 17-\mathrm{H}), 3.77(\mathrm{~s}, 3 \mathrm{H}$, $\left.3-\mathrm{OCH}_{3}\right), 4.46\left(\mathrm{~s}, 2 \mathrm{H}, 16 \mathrm{a}-\mathrm{H}_{2}\right), 6.62(\mathrm{~d}, 1 \mathrm{H}, J=2.0 \mathrm{~Hz}, 4-\mathrm{H}), 6.70(\mathrm{dd}, 1 \mathrm{H}, J=8.5 \mathrm{~Hz}, J=2.0$ $\mathrm{Hz}, 2-\mathrm{H}), 7.19$ (d, $1 \mathrm{H}, J=8.5 \mathrm{~Hz}, 1-\mathrm{H}) .{ }^{13} \mathrm{C}$ NMR $\left(\delta, \mathrm{ppm}, \mathrm{CDCl}_{3}\right): 11.9(\mathrm{C}-18), 26.0$ and 26.1 (C-2" and -6", C-3" and -5"), 27.2, 28.3, 29.7, 36.6, 38.4, 43.9, 44.3 (C-13), 48.4, 55.2 (3-OCH 3$)$, 62.1 (C-1”), 62.1 (C-16a), 85.2 (C-17), 111.5 (C-2), 113.8 (C-4), 126.2 (C-1), 132.3 (C-10), 137.8 (C-5), 157.4 (C-3).

2.3.10. 3-Methoxy-16a-(4'-phenyl-1'H-1',2',3'-triazol-1'-yl)methylestra-1,3,5(10)-trien-17ß-ol (22d)

Compound $14(342 \mathrm{mg}, 1 \mathrm{mmol})$ and phenylacetylene $(2 \mathrm{mmol}, 0.22 \mathrm{ml})$ were used for the synthesis as described in Section 2.3. The crude product was chromatographed on silica gel ethyl acetate $/ \mathrm{CH}_{2} \mathrm{Cl}_{2}(5: 95 \mathrm{v} / \mathrm{v})$ to yield pure $22 \mathrm{~d}$ (368 $\left.\mathrm{mg}, 82 \%\right)$ as a white solid. Mp: $204-205{ }^{\circ} \mathrm{C}$; $R_{\mathrm{f}}=0.38$ (ss B). (Found C, 75.63; H, 7.72. $\mathrm{C}_{28} \mathrm{H}_{33} \mathrm{~N}_{3} \mathrm{O}_{2}$ (443.58) requires C, 75.81; H, 7.50\%). ${ }^{1} \mathrm{H}$ NMR $\left.(\delta, p p m, \text { DMSO-d })_{6}\right): 0.73\left(\mathrm{~s}, 3 \mathrm{H}, 18-\mathrm{H}_{3}\right), 2.73\left(\mathrm{~m}, 2 \mathrm{H}, 6-\mathrm{H}_{2}\right), 3.67\left(\mathrm{~s}, 3 \mathrm{H}, 3-\mathrm{OCH}_{3}\right), 4.36(\mathrm{t}$, $\left.1 \mathrm{H}, J=13.5 \mathrm{~Hz}, 16 \mathrm{a}-\mathrm{H}_{2}\right), 4.54\left(\mathrm{dd}, 1 \mathrm{H}, J=13.5 \mathrm{~Hz}, J=4.0 \mathrm{~Hz}, 16 \mathrm{a}-\mathrm{H}_{2}\right), 4.91(\mathrm{~d}, 1 \mathrm{H}, J=4.0 \mathrm{~Hz}$, 17-H), 6.58 (s, 1H, 4-H), $6.67(\mathrm{~d}, 1 \mathrm{H}, J=8.5 \mathrm{~Hz}, 2-\mathrm{H}), 7.15(\mathrm{~d}, 1 \mathrm{H}, J=8.5 \mathrm{~Hz}, 1-\mathrm{H}), 7.32(\mathrm{t}, 1 \mathrm{H}$, 
$J=7.0 \mathrm{~Hz}, 4$ "'-H), 7.44 (t, 2H, $J=7.0 \mathrm{~Hz}, 3$ "- and 5"-H), $7.86(\mathrm{~d}, 2 \mathrm{H}, J=7.0 \mathrm{~Hz}, 2$ "- and 6"-H), 8.61 (s, 1H, 5'-H). ${ }^{13} \mathrm{C}$ NMR ( $\delta$, ppm, DMSO-d $\mathrm{d}_{6}$ ): 11.8 (C-18), 25.8, 26.7, 27.3, 29.1, 36.3, 38.1, 43.4, 43.5, 43.8, 47.5, 53.5 (C-13), 54.8 (3- $\left.\mathrm{OCH}_{3}\right), 83.1$ (C-17), 111.4 (C-2), 113.3 (C-4), 121.4 (C-5'), 125.0 (C-2" and -6”), 126.0 (C-1), 127.6 (C-4”), 128.8 (C-3" and -5"), 130.8 (C-1"), 132.0 (C-10), 137.3 (C-5), 146.1 (C-4'), 156.9 (C-3).

\subsubsection{3-Methoxy-16a-[4'(4' '-nitro-benzoyloxymethyl)-1 'H-1 ',2',3'-triazol-1 '-yl]methylestra- 1,3,5(10)-trien-17ß-ol (22e)}

Compound 14 (342 mg, $1 \mathrm{mmol}$ ) and propargyl 4-nitrobenzoate ( $2 \mathrm{mmol}, 410 \mathrm{mg}$ ) were used for the synthesis as described in Section 2.3. The crude product was chromatographed on silica gel with ethyl acetate $/ \mathrm{CH}_{2} \mathrm{Cl}_{2}(5: 95 \mathrm{v} / \mathrm{v})$ to yield pure $22 \mathrm{e}(445 \mathrm{mg}, 81 \%)$ as a yellow solid. Mp: $86-88^{\circ} \mathrm{C} ; R_{\mathrm{f}}=0.28$ (ss B). (Found C, 66.08; H, 6.43. $\mathrm{C}_{30} \mathrm{H}_{34} \mathrm{~N}_{4} \mathrm{O}_{6}$ (546.61) requires C, 65.92; H, 6.27\%). ${ }^{1} \mathrm{H}$ NMR ( $\delta$, ppm, DMSO- $\left.\mathrm{d}_{6}\right): 0.69$ (s, 3H, 18- $\left.\mathrm{H}_{3}\right), 2.68\left(\mathrm{~m}, 2 \mathrm{H}, 6-\mathrm{H}_{2}\right), 3.57$ (s, 3H, 3$\left.\mathrm{OCH}_{3}\right), 4.38\left(\mathrm{dd}, 1 \mathrm{H}, J=13.5 \mathrm{~Hz}, J=9.0 \mathrm{~Hz}, 16 \mathrm{a}-\mathrm{H}_{2}\right), 4.52(\mathrm{dd}, 1 \mathrm{H}, J=13.5 \mathrm{~Hz}, J=4.5 \mathrm{~Hz}$, $\left.16 \mathrm{a}-\mathrm{H}_{2}\right), 4.86(\mathrm{~d}, 1 \mathrm{H}, J=4.5 \mathrm{~Hz}, 17-\mathrm{H}), 5.46$ (s, 2H, 4'- $\left.\mathrm{H}_{2}\right), 6.55$ (d, 1H, $\left.J=1.5 \mathrm{~Hz}, 4-\mathrm{H}\right), 6.63$ (dd, $1 \mathrm{H}, J=8.5 \mathrm{~Hz}, 2-\mathrm{H}), 7.10$ (d, $1 \mathrm{H}, J=8.5 \mathrm{~Hz}, 1-\mathrm{H}), 8.16$ (d, 2H, $J=8.5 \mathrm{~Hz}, 3$ "- and 5"-H), $8.28\left(\mathrm{~d}, 2 \mathrm{H}, J=8.5 \mathrm{~Hz}, 2\right.$ "'- and 6 '-H), $8.31\left(\mathrm{~s}, 1 \mathrm{H}, 5\right.$ '-H) ${ }^{13} \mathrm{C}$ NMR $\left(\delta, \mathrm{ppm}, \mathrm{DMSO}-\mathrm{d}_{6}\right): 11.7$ (C-18), 25.7, 26.6, 27.1, 29.0, 36.4, 38.0, 43.3, 43.4 (C-13), 43.7, 47.7, 53.1 (C-16a), 54.7 (3$\left.\mathrm{OCH}_{3}\right), 58.6$ (4"- $\left.\mathrm{CH}_{2}\right), 82.8$ (C-17), 111.3 (C-2), 113.3 (C-4), 123.8 (C-2" and -6"), 125.2 (C-5'), 125.9 (C-1), 130.6 (C-3" and -5”), 131.8 (C-10), 134.7 (C-1'), 137.2 (C-5), 141.1 (C-4”), 150.2 (C-4'), $156.9(\mathrm{C}-3), 163.9(\mathrm{C}=\mathrm{O})$.

2.3.12. 3-Methoxy-16a-(4'-hydroxymethyl-1'H-1',2'3'-triazol-1'-yl)methylestra-1,3,5(10)-trien$17 \beta-o l(22 f)$

Compound 22e (274 mg, $0.5 \mathrm{mmol})$ was dissolved in methanol $(10 \mathrm{ml})$ containing $\mathrm{NaOCH}_{3}(14$ $\mathrm{mg}, 0.25 \mathrm{mmol}$ ), and the solution was allowed to stand for $24 \mathrm{~h}$. It was then diluted with water, and the precipitate separating out was filtered off and recrystallized from a mixture of ethyl acetate/hexane to afford $22 \mathrm{f}(175 \mathrm{mg}, 88 \%)$ as a white crystalline product. Mp: $98-100{ }^{\circ} \mathrm{C} ; R_{\mathrm{f}}=$ 0.28 (ss B). (Found C, 69.74; H, 7.72. $\mathrm{C}_{23} \mathrm{H}_{31} \mathrm{~N}_{3} \mathrm{O}_{3}$ (397.51) requires $\mathrm{C}, 69.49 ; \mathrm{H}, 7.86 \%$ ). ${ }^{1} \mathrm{H}$ $\operatorname{NMR}\left(\delta, \mathrm{ppm}, \mathrm{CDCl}_{3}\right): 0.81\left(\mathrm{~s}, 3 \mathrm{H}, 18-\mathrm{H}_{3}\right), 2.82\left(\mathrm{~m}, 2 \mathrm{H}, 6-\mathrm{H}_{2}\right), 3.50(\mathrm{~d}, 1 \mathrm{H}, J=7.0 \mathrm{~Hz}, 17-\mathrm{H})$, 
$3.76\left(\mathrm{~s}, 3 \mathrm{H}, 3-\mathrm{OCH}_{3}\right), 4.42\left(\mathrm{~d}, 2 \mathrm{H}, J=7.0 \mathrm{~Hz}, 16 \mathrm{a}-\mathrm{H}_{2}\right), 4.71\left(\mathrm{~s}, 2 \mathrm{H}, 4^{\prime}-\mathrm{H}_{2}\right), 6.61(\mathrm{~s}, 1 \mathrm{H}, 4-\mathrm{H})$, $6.69(\mathrm{~d}, 1 \mathrm{H}, J=8.5 \mathrm{~Hz}, 2-\mathrm{H}), 7.17(\mathrm{~d}, 1 \mathrm{H}, J=8.5 \mathrm{~Hz}, 1-\mathrm{H}), 7.68\left(\mathrm{~s}, 1 \mathrm{H}, 5^{\prime}-\mathrm{H}\right) .{ }^{13} \mathrm{C}$ NMR $(\delta$, ppm, $\mathrm{CDCl}_{3}$ ): 11.9 (C-18), 26.1, 27.2, 28.2, 29.6, 36.5, 38.4, 43.8, 44.0, 44.4 (C-13), 48.2, 54.6 (C-16a), 55.2 (3-OCH $\left.{ }_{3}\right), 56.0\left(4\right.$ '- $\left.\mathrm{CH}_{2}\right), 85.1$ (C-17), 111.5 (C-2), 113.8 (C-4), 126.3 (C-1), 132.3 (C-10), 137.8 (C-5), 157.4 (C-3).

2.3.13. 3-Methoxy-16a-(4'-cyclopropyl-1'H-1',2',3'-triazol-1'-yl)methylestra-1,3,5(10)-trien$17 \beta-o l(23 a)$

Compound 15 (342 mg, $1 \mathrm{mmol}$ ) and cyclopropylacetylene ( $2 \mathrm{mmol}, 0.22 \mathrm{ml})$ were used for the synthesis as described in Section 2.3. The crude product was chromatographed on silica gel with ethyl acetate $/ \mathrm{CH}_{2} \mathrm{Cl}_{2}(1: 99 \mathrm{v} / \mathrm{v})$ to yield pure $23 \mathbf{a}(261 \mathrm{mg}, 64 \%)$ as a white solid. Mp: $67-69{ }^{\circ} \mathrm{C}$; $R_{\mathrm{f}}=0.32$ (ss B). (Found C, 73.85; H, 8.32. $\mathrm{C}_{25} \mathrm{H}_{33} \mathrm{~N}_{3} \mathrm{O}_{2}$ (407.55) requires C, 73.68; H, 8.16\%). ${ }^{1} \mathrm{H}$ $\operatorname{NMR}\left(\delta, \mathrm{ppm}, \mathrm{CDCl}_{3}\right): 0.82\left(\mathrm{~m}, 5 \mathrm{H}, 18-\mathrm{H}_{3}\right.$ and cyclopropyl- $\left.\mathrm{H}_{2}\right), 0.95\left(\mathrm{~m}, 2 \mathrm{H}\right.$, cyclopropyl- $\left.\mathrm{H}_{2}\right)$, $2.83\left(\mathrm{~m}, 2 \mathrm{H}, 6-\mathrm{H}_{2}\right), 3.53(\mathrm{~d}, 1 \mathrm{H}, J=7.5 \mathrm{~Hz}, 17-\mathrm{H}), 3.77$ (s, 3H, 3-OCH $\left.{ }_{3}\right), 4.35$ (t, $1 \mathrm{H}, J=7.5 \mathrm{~Hz}$, $\left.16 \mathrm{a}-\mathrm{H}_{2}\right), 4.44\left(\mathrm{dd}, 1 \mathrm{H}, J=13.5 \mathrm{~Hz}, J=7.5 \mathrm{~Hz}, 16 \mathrm{a}-\mathrm{H}_{2}\right), 6.62(\mathrm{~d}, 1 \mathrm{H}, J=2.0 \mathrm{~Hz}, 4-\mathrm{H}), 6.70(\mathrm{dd}$, $1 \mathrm{H}, J=8.5 \mathrm{~Hz}, J=2.0 \mathrm{~Hz}, 2-\mathrm{H}), 7.18(\mathrm{~d}, 1 \mathrm{H}, J=8.5 \mathrm{~Hz}, 1-\mathrm{H}) .{ }^{13} \mathrm{C} \mathrm{NMR}\left(\delta, \mathrm{ppm}, \mathrm{CDCl}_{3}\right): 6.7$ (C-1"), 7.7 (C-2" and -3"), 11.8 (C-18), 26.1, 27.2, 28.2, 29.7, 36.6, 38.4, 43.9, 44.3, 44,3 (C16a), 48.3, 54.5 (C-13), 62.1 (3-OCH ${ }_{3}$ ), 85.1 (C-17), 111.5 (C-2), 113.8 (C-4), 126.2 (C-1), 132.3 (C-10), 137.8 (C-5), $157.4(\mathrm{C}-3)$.

2.3.14. 3-Methoxy-16ß-(4'-cyclopentyl-1'H-1',2',3'-triazol-1-yl)methylestra-1,3,5(10)-trien-17aol $(23 b)$

Compound 15 (342 mg, $1 \mathrm{mmol}$ ) and cyclopentylacetylene ( $2 \mathrm{mmol}, 0.22 \mathrm{ml}$ ) were used for the synthesis as described in Section 2.3. The crude product was chromatographed on silica gel with ethyl acetate/ $\mathrm{CH}_{2} \mathrm{Cl}_{2}(1: 99 \mathrm{v} / \mathrm{v})$ to yield pure $\mathbf{2 3 b}(380 \mathrm{mg}, 87 \%)$ as yellow crystalline material. Mp: 67-68 ${ }^{\circ} \mathrm{C} ; R_{\mathrm{f}}=0.36$ (ss B). (Found C, 74.28; H, 8.47. $\mathrm{C}_{27} \mathrm{H}_{37} \mathrm{~N}_{3} \mathrm{O}_{2}$ (435.60) requires C, 74.45; H, 8.56\%). ${ }^{1} \mathrm{H}$ NMR ( $\delta$, ppm, $\left.\mathrm{CDCl}_{3}\right): 0.75$ (s, 3H, 18- $\left.\mathrm{H}_{3}\right), 2.85\left(\mathrm{~m}, 2 \mathrm{H}, 6-\mathrm{H}_{2}\right), 3.68$ (s, $1 \mathrm{H}, 17-\mathrm{H}), 3.77\left(\mathrm{~s}, 3 \mathrm{H}, 3-\mathrm{OCH}_{3}\right), 4.44\left(\mathrm{~d}, 2 \mathrm{H}, J=15.0 \mathrm{~Hz}, 16 \mathrm{a}-\mathrm{H}_{2}\right), 6.62(\mathrm{~s}, 1 \mathrm{H}, 4-\mathrm{H}), 6.70(\mathrm{~d}$, $1 \mathrm{H}, J=8.5 \mathrm{~Hz}, 2-\mathrm{H}), 7.20(\mathrm{t}, 1 \mathrm{H}, J=8.5 \mathrm{~Hz}, 1-\mathrm{H}) .{ }^{13} \mathrm{C} \mathrm{NMR}\left(\delta, \mathrm{ppm}, \mathrm{CDCl}_{3}\right): 17.9(\mathrm{C}-18), 25.1$ (C-3" and -4"), 25.9, 26.1, 27.2, 28.0, 29.7, 30.4, 31.8, 36.6 (C-16a), 38.5, 43.3, 43.8, 45.1 (C- 
13), 48.9, 55.2 (3- $\left.\mathrm{OCH}_{3}\right), 62.1$ (C-1"), 82.6 (C-17), 111.5 (C-2), 113.7 (C-4), 113.8 (C-5'), 126.2 (C-1), 132.1 (C-10), 137.8 (C-5), 137.8 (C-4'), 157.4 (C-3).

2.3.15. 3-Methoxy-16ß-(4'-cyclohexyl-1 'H-1',2',3'-triazol-1'-yl)methyestra-1,3,5(10)-trien-17aol (23c)

Compound 15 (342, $1 \mathrm{mmol})$ and cyclohexylacetylene $(2 \mathrm{mmol}, 0.22 \mathrm{ml})$ were used for the synthesis as described in Section 2.3. The crude product was chromatographed on silica gel with ethyl acetate $/ \mathrm{CH}_{2} \mathrm{Cl}_{2}(5: 95 \mathrm{v} / \mathrm{v})$ to yield pure $23 \mathrm{c}(306 \mathrm{mg}, 68 \%)$ as a white solid. Mp: $90-92{ }^{\circ} \mathrm{C}$; $R_{\mathrm{f}}=0.37$ (ss B). (Found C, 74.95; H, 8.83. $\mathrm{C}_{28} \mathrm{H}_{41} \mathrm{~N}_{3} \mathrm{O}_{2}$ (449.63) requires C, 74.80; H, 8.74\%). ${ }^{1} \mathrm{H}$ NMR ( $\delta$, ppm, $\left.\mathrm{CDCl}_{3}\right): 0.75\left(\mathrm{~s}, 3 \mathrm{H}, 18-\mathrm{H}_{3}\right), 2.84\left(\mathrm{~m}, 2 \mathrm{H}, 6-\mathrm{H}_{2}\right), 3.67(\mathrm{~d}, 1 \mathrm{H}, J=1.0 \mathrm{~Hz}, 17-\mathrm{H})$, $3.77\left(\mathrm{~S}, 3 \mathrm{H}, 3-\mathrm{OCH}_{3}\right), 4.43\left(\mathrm{~m}, 1 \mathrm{H}, 16 \mathrm{a}-\mathrm{H}_{2}\right), 6.62(\mathrm{~d}, 1 \mathrm{H}, J=2.5 \mathrm{~Hz}, 4-\mathrm{H}), 6.71(\mathrm{dd}, 1 \mathrm{H}, J=8.5$ $\mathrm{Hz}, J=2.5 \mathrm{~Hz}, 2-\mathrm{H}), 7.20(\mathrm{t}, 1 \mathrm{H}, J=8.5 \mathrm{~Hz}, 1-\mathrm{H}), 7.35\left(\mathrm{~s}, 1 \mathrm{H}, 5^{\prime}-\mathrm{H}\right) .{ }^{13} \mathrm{C} \mathrm{NMR}(\delta, \mathrm{ppm}$, $\mathrm{CDCl}_{3}$ ): 17.9 (C-18), 25.9, 26.0, 26.1 (C-2" and -6"), 28.0, 29.7, 30.4, 31.8, 33.0, 35.2 (C-1"), 36.6, 38.5, 43.3, 45.1 (C-13), 48.9, 49.1, 54.3 (C-16a), $55.2\left(3-\mathrm{OCH}_{3}\right), 82.6(\mathrm{C}-1), 132.4(\mathrm{C}-10)$, 137.8 (C-5), 153.7 (C-4'), 157.7 (C-3).

2.3.16. 3-Methoxy-168-(4'-phenyl-1'H-1',2',3'-triazol-1'-yl)methy-estra-1,3,5(10)-trien-17a-ol (23d)

Compound $15(342 \mathrm{mg}, 1 \mathrm{mmol})$ and phenylacetylene $(2 \mathrm{mmol}, 0.22 \mathrm{ml})$ were used for the synthesis as described in Section 2.3. The crude product was chromatographed on silica gel with ethyl acetate $/ \mathrm{CH}_{2} \mathrm{Cl}_{2}(2.5: 97.5 \mathrm{v} / \mathrm{v})$ to yield pure $\mathbf{2 3 d}(299 \mathrm{mg}, 67 \%)$ as white crystals. Mp: $173-174{ }^{\circ} \mathrm{C} ; R_{\mathrm{f}}=0.34$ (ss B). (Found C 75.98; H, 7.33. $\mathrm{C}_{28} \mathrm{H}_{33} \mathrm{~N}_{3} \mathrm{O}_{2}$ (443.58) requires $\mathrm{C}, 75.81$; H, 7.50\%). ${ }^{1} \mathrm{H}$ NMR ( $\left.\delta, p p m, \mathrm{CDCl}_{3}\right): 0.79$ (s, 3H, 18- $\left.\mathrm{H}_{3}\right), 2.85\left(\mathrm{~m}, 2 \mathrm{H}, 6-\mathrm{H}_{2}\right), 3.71(\mathrm{~d}, 1 \mathrm{H}, J=$ $1.5 \mathrm{~Hz}, 17-\mathrm{H}), 3.78$ (s, 3H, 3- $\left.\mathrm{OCH}_{3}\right), 4.46\left(\mathrm{dd}, 1 \mathrm{H}, J=13.5 \mathrm{~Hz}, J=8.0 \mathrm{~Hz}, 16 \mathrm{a}-\mathrm{H}_{2}\right), 4.55$ (dd, $\left.1 \mathrm{H}, J=13.5 \mathrm{~Hz}, J=8.0 \mathrm{~Hz}, 16 \mathrm{a}-\mathrm{H}_{2}\right), 6.63(\mathrm{~d}, 1 \mathrm{H}, J=2.0 \mathrm{~Hz}, 4-\mathrm{H}), 6.72(\mathrm{dd}, 1 \mathrm{H}, J=8.5 \mathrm{~Hz}, J=$ $2.5 \mathrm{~Hz}, 2-\mathrm{H}), 7.21$ (d, $1 \mathrm{H}, J=8.5 \mathrm{~Hz}, 1-\mathrm{H}), 7.27$ (t, $1 \mathrm{H} J=7.5 \mathrm{~Hz}, 4$ "-H), 7.42 (t, 2H, $J=7.5 \mathrm{~Hz}$, 3"- and 5"-H), $7.83\left(\mathrm{~d}, 2 \mathrm{H}, J=7.5 \mathrm{~Hz}, 2\right.$ "- and 6"-H), 7.87 (s, 1H, 5'-H). ${ }^{13} \mathrm{C}$ NMR $(\delta$, ppm, $\left.\mathrm{CDCl}_{3}\right): 17.9$ (C-18), 25.9, 27.9, 29.7, 30.4, 31.8, 38.5, 43.3, 45.1, (C-13), 48.8, 49.1, 54.5 (C16a), $55.2\left(3-\mathrm{OCH}_{3}\right), 82.5$ (C-17), 111.5 (C-2), 113.7 (C-4), 119.6 (C-5'), 125.7 (C-2" and -6"), 126.3 (C-1), 128.1 (C-4”), 128.8 (C-3" and -5”), 130.5 (C-1"), 132.4 (C-10), 137.8 (C-5), 147.8 (C-4'), $157.4(\mathrm{C}-3)$. 


\subsubsection{3-Methoxy-16ß-[4'(4"'-nitro-benzoyloxymethyl)-1 'H-1',2',3'-triazol-1 '-yl)methylestra-} 1,3,5(10)-trien-17a-ol (23e)

Compound 15 (342, $1 \mathrm{mmol})$ and propargyl 4-nitro benzoate $(2 \mathrm{mmol}, 410 \mathrm{mg})$ were used for the synthesis as described in Section 2.3. The crude product was chromatographed on silica gel with ethyl acetate $/ \mathrm{CH}_{2} \mathrm{Cl}_{2}(5: 95 \mathrm{v} / \mathrm{v})$ to yield pure $23 \mathrm{e}(370 \mathrm{mg}, 67 \%)$ as a yellow crystalline material. Mp: $62-63{ }^{\circ} \mathrm{C} ; R_{\mathrm{f}}=0.38$ (ss B). (Found C, 66.14; H, 6.42. $\mathrm{C}_{30} \mathrm{H}_{34} \mathrm{~N}_{4} \mathrm{O}_{6}(546.61$ ) requires $\mathrm{C}$, 65.92; H, 6.27\%). ${ }^{1} \mathrm{H}$ NMR ( $\delta$, ppm, DMSO-d $\left.\mathrm{d}_{6}\right): 0.65\left(\mathrm{~s}, 3 \mathrm{H}, 18-\mathrm{H}_{3}\right), 2.74\left(\mathrm{~m}, 2 \mathrm{H}, 6-\mathrm{H}_{2}\right), 3.68(\mathrm{~s}$, $\left.3 \mathrm{H}, 3-\mathrm{OCH}_{3}\right), 4.41\left(\mathrm{dd}, 1 \mathrm{H}, J=13.0 \mathrm{~Hz}, J=8.5 \mathrm{~Hz}, 16 \mathrm{a}-\mathrm{H}_{2}\right), 4.56(\mathrm{dd}, 1 \mathrm{H}, J=13.0 \mathrm{~Hz}, J=8.5$ Hz, 16a- $\left.\mathrm{H}_{2}\right), 4.63$ (d, 1H, J = 4.5 Hz, 17-H), 6.58 (s, 1H, 4-H), 6.66 (d, 1H, J = 8.5 Hz, 2-H), 7.16 (d, $1 \mathrm{H}, J=8.5 \mathrm{~Hz}, 1-\mathrm{H}), 8.19$ (d, 2H, $J=8.5 \mathrm{~Hz}, 3$ "- and 5"-H), 8.34 (d, 2H, $J=8.5 \mathrm{~Hz}, 2$ "- and 6"-H). ${ }^{13} \mathrm{C}$ NMR ( $\delta$, ppm, DMSO-d 6 ): 17.5 (C-18), 25.6, 27.5, 29.6, 31.8, 38.2, 43.0, 44.5, 47.9 (C-13), 48.2, 49.1, 53.6 (C-16a), $54.8\left(3-\mathrm{OCH}_{3}\right), 58.7\left(4^{\prime}-\mathrm{CH}_{2}\right), 80.8$ (C-17), 111.3 (C-2), 113.3 (C-4), 123.8 (C-1), 126.1 (C-5'), 130.6 (C-2" and -6"), 131.9 (C-3" and -5"), 133.0 (C-10), 134.7 (C-1”), 137.3 (C-5), 141.4 (C-4”), 150.2 (C-4'), 156.9 (C-3), 163.9 (C=O).

\subsubsection{3-Methoxy-16ß-(4'-hydroxymethyl-1 'H-1',2'3'-triazol-1'-yl)methylestra-1,3,5(10)-trien-} $17 a-o l(23 f)$

Compound 23e (274 mg, $0.5 \mathrm{mmol})$ was dissolved in methanol $(10 \mathrm{ml})$ containing $\mathrm{NaOCH}_{3}(14$ $\mathrm{mg}, 0.25 \mathrm{mmol}$ ), and the solution was allowed to stand for $24 \mathrm{~h}$. It was then diluted with water, and the precipitate separating out was filtered off, dissolved in dichloromethane and washed with water. The organic phase was dried over $\mathrm{Na}_{2} \mathrm{SO}_{4}$, and evaporated in vacuo to afford $\mathbf{2 3 f}$ (183 $\mathrm{mg}$, $92 \%$ ) as oil. $R_{\mathrm{f}}=0.26$ (ss B). (Found $\mathrm{C}, 69.28 ; \mathrm{H}, 7.95 . \mathrm{C}_{23} \mathrm{H}_{31} \mathrm{~N}_{3} \mathrm{O}_{3}$ (397.51) requires $\mathrm{C}, 69.49$; $\mathrm{H}, 7.86 \%) .{ }^{1} \mathrm{H}$ NMR $\left(\delta, \mathrm{ppm}, \mathrm{CDCl}_{3}\right): 0.78\left(\mathrm{~s}, 3 \mathrm{H}, 18-\mathrm{H}_{3}\right), 2.85\left(\mathrm{~m}, 2 \mathrm{H}, 6-\mathrm{H}_{2}\right), 3.65(\mathrm{~s}, 1 \mathrm{H}, 17-$ H), $3.77\left(\mathrm{~s}, 3 \mathrm{H}, 3-\mathrm{OCH}_{3}\right), 4.46\left(\mathrm{~m}, 2 \mathrm{H}, 16 \mathrm{a}-\mathrm{H}_{2}\right), 4.78\left(\mathrm{~s}, 2 \mathrm{H}, 4\right.$ '- $\left.\mathrm{H}_{2}\right), 6.62(\mathrm{~d}, 1 \mathrm{H}, J=2.0 \mathrm{~Hz}, 4-$ H), $6.72(\mathrm{dd}, 1 \mathrm{H}, J=8.5 \mathrm{~Hz}, J=2.5 \mathrm{~Hz}, 2-\mathrm{H}), 7.19(\mathrm{~d}, 1 \mathrm{H}, J=8.5 \mathrm{~Hz}, 1-\mathrm{H}) .{ }^{13} \mathrm{C}$ NMR $(\delta, \mathrm{ppm}$, $\mathrm{CDCl}_{3}$ ): 17.9 (C-18), 25.9, 27.9, 29.7, 30.3, 31.8, 38.5, 43.3, 45.2 (C-13), 48.8, 49.2, 54.6 (C16a), $55.2\left(3-\mathrm{OCH}_{3}\right), 56.1$ (4'- $\left.\mathrm{CH}_{2}\right), 82.1$ (C-17), 111.5 (C-2), 113.7 (C-4), 123.5 (C-5'), 126.3 (C-1), 132.4 (C-10), 137.8 (C-5), 157.4 (C-3). 


\subsubsection{3-Methoxy-16a-(4'-cyclopropyl-1 'H-1 ',2',3'-triazol-1'-yl)methylestra-1,3,5(10)-trien-}

$17 a-o l(24 a)$

Compound 16 (342 mg, $1 \mathrm{mmol})$ and cyclopropylacetylene $(2 \mathrm{mmol}, 0.22 \mathrm{ml})$ were used for the synthesis as described in Section 2.3. The crude product was chromatographed on silica gel with ethyl acetate $/ \mathrm{CH}_{2} \mathrm{Cl}_{2}(2.5: 97.5 \mathrm{v} / \mathrm{v})$ to yield pure $24 \mathbf{a}(310 \mathrm{mg}, 76 \%)$ as a white solid. $\mathrm{Mp}$ : $165-166{ }^{\circ} \mathrm{C} ; R_{\mathrm{f}}=0.40$ (ss B). (Found C, 73.85; H, 8.34. $\mathrm{C}_{25} \mathrm{H}_{33} \mathrm{~N}_{3} \mathrm{O}_{2}$ (407.55) requires $\mathrm{C}, 73.68$; $\mathrm{H}, 8.16 \%) .{ }^{1} \mathrm{H}$ NMR $\left(\delta, \mathrm{ppm}, \mathrm{CDCl}_{3}\right): 0.74\left(\mathrm{~s}, 3 \mathrm{H}, 18-\mathrm{H}_{3}\right), 0.85$ and $0.96(2 \mathrm{x} \mathrm{m}, 4 \mathrm{H}, 2$ "- and 3 "$\left.\mathrm{H}_{2}\right), 2.85\left(\mathrm{~m}, 2 \mathrm{H}, 6-\mathrm{H}_{2}\right), 3.63(\mathrm{~d}, 1 \mathrm{H}, J=5.0 \mathrm{~Hz}, 17-\mathrm{H}), 3.77\left(\mathrm{~s}, 3 \mathrm{H}, 3-\mathrm{OCH}_{3}\right), 4.28(\mathrm{dd}, 1 \mathrm{H}, J=$ $\left.13.0 \mathrm{~Hz}, J=5.0 \mathrm{~Hz}, 16 \mathrm{a}-\mathrm{H}_{2}\right), 4.59\left(\mathrm{t}, 1 \mathrm{H}, J=12.0 \mathrm{~Hz}, 16 \mathrm{a}-\mathrm{H}_{2}\right), 6.63(\mathrm{~d}, 1 \mathrm{H}, J=2.0 \mathrm{~Hz}, 4-\mathrm{H})$, $6.71(\mathrm{dd}, 1 \mathrm{H}, J=8.5 \mathrm{~Hz}, J=2.5 \mathrm{~Hz}, 2-\mathrm{H}), 7.22(\mathrm{~d}, 1 \mathrm{H}, J=8.5 \mathrm{~Hz}, 1-\mathrm{H}) \cdot{ }^{13} \mathrm{C}$ NMR $(\delta, \mathrm{ppm}$, $\mathrm{CDCl}_{3}$ ): 6.6 (C-1"), 7.7 and 7.8 (C-2" and -3"), 17.1 (C-18), 26.0, 28.0, 28.9, 29.8, 31.2, 38.9, 42.3, 46.3 (C-16a), 47.0, 50.5 (C-13), $55.2\left(3-\mathrm{OCH}_{3}\right), 78.8$ (C-17), 111.4 (C-2), 113.7 (C-4), 120.6 (C-5'), 126.3 (C-1), 132.5 (C-10), 137.9 (C-5), 149.8 (C-4'), 157.4 (C-3).

2.3.20. 3-Methoxy-16a-(4'-cyclopentyl-1 'H-1',2',3'-triazol-1'-yl)methyl-estra-1,3,5(10)-trien$17 a-o l(24 b)$

Compound 16 (342 mg, $1 \mathrm{mmol}$ ) and cyclopentylacetylene $(2 \mathrm{mmol}, 0.22 \mathrm{ml})$ were used for the synthesis as described in Section 2.3. The crude product was chromatographed on silica gel with ethyl acetate/ $\mathrm{CH}_{2} \mathrm{Cl}_{2}(1: 99 \mathrm{v} / \mathrm{v})$ to yield pure $\mathbf{2 4 b}(383 \mathrm{mg}, 88 \%)$ as yellow crystalline product. Mp: $171-173{ }^{\circ} \mathrm{C} ; R_{\mathrm{f}}=0.42$ (ss B). (Found C, 74.67; H, 8.72. $\mathrm{C}_{27} \mathrm{H}_{37} \mathrm{~N}_{3} \mathrm{O}_{2}$ (435.60) requires $\mathrm{C}$, 74.45; H, 8.56\%). ${ }^{1} \mathrm{H}$ NMR ( $\delta$, ppm, $\mathrm{CDCl}_{3}$ ): 075 (s, 3H, 18- $\left.\mathrm{H}_{3}\right), 1.25$ (s, 8H, 2"-, 3"-, 4"- and 5"- $\left.\mathrm{H}_{2}\right), 2.86\left(\mathrm{~m}, 2 \mathrm{H}, 6-\mathrm{H}_{2}\right), 3.18(\mathrm{~m}, 1 \mathrm{H}, 1$ "- $\mathrm{H}), 3.64(\mathrm{~d}, 1 \mathrm{H}, J=5.0 \mathrm{~Hz}, 17-\mathrm{H}), 3.77(\mathrm{~s}, 3 \mathrm{H}, 3-$ $\left.\mathrm{OCH}_{3}\right), 4.29\left(\mathrm{dd}, 1 \mathrm{H}, J=13.5 \mathrm{~Hz}, J=5.5 \mathrm{~Hz}, 16 \mathrm{a}-\mathrm{H}_{2}\right), 4.62(\mathrm{dd}, 1 \mathrm{H}, J=13.5 \mathrm{~Hz}, \mathrm{~J}=11.5 \mathrm{~Hz}$, $\left.16 \mathrm{a}-\mathrm{H}_{2}\right), 6.63(\mathrm{~d}, 1 \mathrm{H}, J=2.0 \mathrm{~Hz}, 4-\mathrm{H}), 6.71(\mathrm{dd}, 1 \mathrm{H}, J=8.5 \mathrm{~Hz}, J=2.0 \mathrm{~Hz}, 2-\mathrm{H}), 7.22(\mathrm{~d}, 1 \mathrm{H}, J$ $=8.5 \mathrm{~Hz}, 1-\mathrm{H}), 7.36$ (s, $\left.1 \mathrm{H}, 5^{\prime}-\mathrm{H}\right) .{ }^{13} \mathrm{C}$ NMR $\left(\delta, \mathrm{ppm}, \mathrm{CDCl}_{3}\right): 17.2$ (C-18), 25.1 (C-3" and -4"), 26.0, 28.0, 29.0, 29.7, 29.9, 31.2, 33.2, 36.7, 38.9, 42.4, 43.5, 46.3 (C-13), 47.0 (C-1"), 50.5 (C16a), $55.2\left(3-\mathrm{OCH}_{3}\right), 78.8$ (C-17), 111.4 (C-2), 113.8 (C-4), 120.6 (C-5'), 126.3 (C-1), 132.6 (C10), 137.9 (C-5), 152.3 (C-4'), 157.4 (C-3).

2.3.21. 3-Methoxy-16a-(4'-cyclohexyl-1'H-1',2',3'-triazol-1'-yl)methylestra-1,3,5(10)-trien-17aol $(24 c)$ 
Compound 16 (342 mg, $1 \mathrm{mmol})$ and cyclohexylacetylene $(2 \mathrm{mmol}, 0.22 \mathrm{ml})$ were used for the synthesis as described in Section 2.3. The crude product was chromatographed on silica gel with ethyl acetate/ $\mathrm{CH}_{2} \mathrm{Cl}_{2}(1: 99 \mathrm{v} / \mathrm{v})$ to yield pure $\mathbf{2 4 c}(162 \mathrm{mg}, 36 \%)$ as yellow crystals. Mp: 208-210 ${ }^{\circ} \mathrm{C} ; R_{\mathrm{f}}=0.42$ (ss B). (Found C, 74.97; H, 8.56. $\mathrm{C}_{28} \mathrm{H}_{41} \mathrm{~N}_{3} \mathrm{O}_{2}$ (449.63) requires $\mathrm{C}, 74.80 ; \mathrm{H}, 8.74 \%$ ). ${ }^{1} \mathrm{H}$ NMR $\left(\delta, \mathrm{ppm}, \mathrm{CDCl}_{3}\right): 0.75$ (s, 3H, 18- $\left.\mathrm{H}_{3}\right), 1.26$ (s, 8H, 2"-, 3"-, 5"- and 6"- $\left.\mathrm{H}_{2}\right), 2.88(\mathrm{~m}, 2 \mathrm{H}$, 6- $\left.\mathrm{H}_{2}\right), 2.90\left(\mathrm{~m}, 2 \mathrm{H}, 4\right.$ "'- $\left.\mathrm{H}_{2}\right), 3.64(\mathrm{~d}, 1 \mathrm{H}, J=5.0 \mathrm{~Hz}, 17-\mathrm{H}), 3.77$ (s, 3H, 3- $\left.\mathrm{OCH}_{3}\right), 4.29$ (dd, $1 \mathrm{H}, J$ $\left.=13.5 \mathrm{~Hz}, J=5.0 \mathrm{~Hz}, 16 \mathrm{a}-\mathrm{H}_{2}\right), 4.62\left(\mathrm{dd}, 1 \mathrm{H}, J=13.5 \mathrm{~Hz}, J=11.0 \mathrm{~Hz}, 16 \mathrm{a}-\mathrm{H}_{2}\right), 6.63(\mathrm{~d}, 1 \mathrm{H}, J=$ $2.0 \mathrm{~Hz}, 4-\mathrm{H}), 6.71$ (dd, 1H, $J=8.5 \mathrm{~Hz}, J=2.5 \mathrm{~Hz}, 2-\mathrm{H}), 7.22$ (d, 1H, $J=8.5 \mathrm{~Hz}, 1-\mathrm{H}), 7.34$ (s, $\left.1 \mathrm{H}, 5^{\prime}-\mathrm{H}\right) .{ }^{13} \mathrm{C}$ NMR ( $\delta$, ppm, $\mathrm{CDCl}_{3}$ ): 17.2 (C-18), 26.0 and 26.1 (C-2", -3", -5" and -6"), 28.0, 29.0, 29.7, 29.8, 31.2, 33.0, 25.2, 38.9, 42.4, 43.5, 46.3 (C-13), 47.0 (C-1”), 50.5 (C-16a), 55.0 (3-OCH $\left.{ }_{3}\right), 78.8$ (C-17), 111.4 (C-2), 113.8 (C-4), 120.2 (C-5'), 126.3 (C-1), 132.6 (C-10), 137.9 (C-5), 153.3 (C-4'), 157.4 (C-3).

\subsubsection{3-Methoxy-16a-(4'-phenyl-1 'H-1',2',3'-triazol-1'-yl)methylestra-1,3,5(10)-trien-17a-ol (24d)}

Compound $16342 \mathrm{mg}, 1 \mathrm{mmol})$ and phenylacetylene $(2 \mathrm{mmol}, 0.22 \mathrm{ml})$ were used for the synthesis as described in Section 2.3. The crude product was chromatographed on silica gel with $\mathrm{CH}_{2} \mathrm{Cl}_{2}$ yield pure $24 \mathrm{~d}$ (394 mg, 89\%) as white solid. Mp: $189.5-191{ }^{\circ} \mathrm{C} ; R_{\mathrm{f}}=0.46$ (ss B). (Found $\mathrm{C}, 75.65 ; \mathrm{H}, 7.67 . \mathrm{C}_{28} \mathrm{H}_{33} \mathrm{~N}_{3} \mathrm{O}_{2}$ (443.58) requires $\left.\mathrm{C}, 75.81 ; \mathrm{H}, 7.50 \%\right) .{ }^{1} \mathrm{H} \mathrm{NMR}\left(\delta, \mathrm{ppm}, \mathrm{CDCl}_{3}\right)$ : $0.75\left(\mathrm{~s}, 3 \mathrm{H}, 18-\mathrm{H}_{3}\right), 2.86\left(\mathrm{~m}, 2 \mathrm{H}, 6-\mathrm{H}_{2}\right), 3.68(\mathrm{~d}, 1 \mathrm{H}, J=5.0 \mathrm{~Hz}, 17-\mathrm{H}), 3.78\left(\mathrm{~s}, 3 \mathrm{H}, 3-\mathrm{OCH}_{3}\right)$, $4.41\left(\mathrm{dd}, 1 \mathrm{H}, J=13.5 \mathrm{~Hz}, J=6.0 \mathrm{~Hz}, 16 \mathrm{a}-\mathrm{H}_{2}\right), 4.69\left(\mathrm{dd}, 1 \mathrm{H}, J=14.5 \mathrm{~Hz}, J=10.5 \mathrm{~Hz}, 16 \mathrm{a}-\mathrm{H}_{2}\right)$, $6.64(\mathrm{~d}, 1 \mathrm{H}, J=2.0 \mathrm{~Hz}, 4-\mathrm{H}), 6.72(\mathrm{dd}, 1 \mathrm{H}, J=8.5 \mathrm{~Hz}, J=2.5 \mathrm{~Hz}, 2-\mathrm{H}), 7.22(\mathrm{~d}, 1 \mathrm{H}, J=8.5 \mathrm{~Hz}$, 1-H), 7.34 (t, 1H, $J=7.5 \mathrm{~Hz}, 4$ "'-H), 7.43 (t, 2H, $J=7.5 \mathrm{~Hz}, 3$ "- and 5"-H), 7.83 (d, 2H, $J=7.5$ $\mathrm{Hz}, 2$ "'- and 6"-H), 7.88 (s, 1H, 5'-H). ${ }^{13} \mathrm{C}$ NMR ( $\delta$, ppm, $\left.\mathrm{CDCl}_{3}\right)$ : 17.1 (C-18), 26.0, 28.0, 29.8, 31.2, 38.9, 42.3, 43.5, 46.4 (C-13), 47.0, 50.7, $55.2\left(3-\mathrm{OCH}_{3}\right), 78.8$ (C-17), 111.5 (C-2), 113.8 (C-4), 120.6 (C-5'), 125.6 (C-2" and -6”), 126.3 (C-1), 128.1 (C-4”), 128.8 (C-3" and -5"), 130.5 (C-1”), 132.5 (C-10), 137.9 (C-5), 147.3 (C-4'), 157.4 (C-3).

2.3.23. 3-Methoxy-16a-[4'-(4'"nitrobenzoyloxymethyl)-1'H-1',2',3'-triazol-1'-yl]methylestra1,3,5(10)-trien-17a-ol (24e) 
Compound 16 (342, $1 \mathrm{mmol})$ and propargyl 4-nitrobenzoate ( $2 \mathrm{mmol}, 210 \mathrm{mg}$ ) were used for the synthesis as described in Section 2.3. The crude product was chromatographed on silica gel with $\mathrm{CH}_{2} \mathrm{Cl}_{2} /$ hexane (1:3, v/v) to yield pure (344 mg, 63\%) as yellow crystals. Mp: $64{ }^{\circ} \mathrm{C} ; R_{\mathrm{f}}=0.45$ (ss B). (Found, C, 66.14; H, 6.05. $\mathrm{C}_{30} \mathrm{H}_{34} \mathrm{~N}_{4} \mathrm{O}_{6}(546.61)$ requires $\left.\mathrm{C}, 65.92 ; \mathrm{H}, 6.27 \%\right) .{ }^{1} \mathrm{H}$ NMR $(\delta$, ppm, $\left.\mathrm{CDCl}_{3}\right): 0.75\left(\mathrm{~s}, 3 \mathrm{H}, 18-\mathrm{H}_{3}\right), 2.84\left(\mathrm{~m}, 2 \mathrm{H}, 6-\mathrm{H}_{2}\right), 3.66(\mathrm{~d}, 1 \mathrm{H}, J=4.5 \mathrm{~Hz}, 17-\mathrm{H}), 3.77$ (s, $\left.3 \mathrm{H}, 3-\mathrm{OCH}_{3}\right), 4.40\left(\mathrm{dd}, 1 \mathrm{H}, J=13.5 \mathrm{~Hz}, J=5.5 \mathrm{~Hz}, 16 \mathrm{a}-\mathrm{H}_{2}\right), 4.66\left(\mathrm{t}, 1 \mathrm{H}, J=13.5 \mathrm{~Hz}, 16 \mathrm{a}-\mathrm{H}_{2}\right)$, $5.53\left(\mathrm{~s}, 2 \mathrm{H}, 4\right.$ ' $\left.-\mathrm{H}_{2}\right), 6.62(\mathrm{t}, 1 \mathrm{H}, J=2.0 \mathrm{~Hz}, 4-\mathrm{H}), 6.71(\mathrm{dd}, 1 \mathrm{H}, J=8.5 \mathrm{~Hz}, J=2.5 \mathrm{~Hz}, 2-\mathrm{H}), 7.20$ (d, 1H, $J=8.5 \mathrm{~Hz}, 1-\mathrm{H}), 7.85$ (s, 1H, 5'-H), 8.22 (d, 2H, $J=9.0 \mathrm{~Hz}, 3$ '- and 5"'-H), 8.72 (d, 2H, $J$ $=9.0 \mathrm{~Hz}, 2$ "- and 6"-H). ${ }^{13} \mathrm{C} \mathrm{NMR}\left(\delta, \mathrm{ppm}, \mathrm{CDCl}_{3}\right): 17.1(\mathrm{C}-18), 22.7,25.9,28.0,29.0,29.8$, 31.2, 38.9, 42.0, 43.5, 46.4 (C-13), 47.0 (4'-CH2), 78.8 (C-17), 111.5 (C-2), 113.8 (C-4), 114.0 (C-1'), 123.5 (C-2” and -6”) 126.3 (C-5'), 130.9 (C-3" and -5”), 135.0 (C-10), 137.8 (C-5), 141.5 (C-4”), $150.6(\mathrm{C}-4$ '), $157.5(\mathrm{C}-3), 164.6(\mathrm{C}=\mathrm{O})$.

\subsubsection{3-Methoxy-16a-(4'-hydroxymethyl-1 'H-1 ',2',3'-triazol-1'-yl)methylestra-1,3,5(10)-trien-} $17 a-o l(24 f)$

Compound 24e (274 mg, $0.5 \mathrm{mmol})$ was dissolved in methanol $(10 \mathrm{ml})$ containing $\mathrm{NaOCH}_{3}(14$ $\mathrm{mg}, 0.25 \mathrm{mmol}$ ), and the solution was allowed to stand for $24 \mathrm{~h}$. It was then diluted with water, and the precipitate separating out was filtered off and recrystallized from a mixture of acetone/hexane to afford $24 \mathbf{f}(187 \mathrm{mg}, 94 \%)$ as a white crystalline product. Mp: $149-150{ }^{\circ} \mathrm{C} ; R_{\mathrm{f}}$ $=0.25$ (ss B). (Found C, 69.55; H, 7.95. $\mathrm{C}_{23} \mathrm{H}_{31} \mathrm{~N}_{3} \mathrm{O}_{3}(397.51)$ requires $\mathrm{C}, 69.49 ; \mathrm{H}, 7.86 \%$ ). ${ }^{1} \mathrm{H}$ $\operatorname{NMR}\left(\delta, \mathrm{ppm}, \mathrm{CDCl}_{3}\right): 0.74\left(\mathrm{~s}, 3 \mathrm{H}, 18-\mathrm{H}_{3}\right), 2.85\left(\mathrm{~m}, 2 \mathrm{H}, 6-\mathrm{H}_{2}\right), 3.62(\mathrm{~d}, 1 \mathrm{H}, J=4.0 \mathrm{~Hz}, 17-\mathrm{H})$, $3.77\left(\mathrm{~s}, 3 \mathrm{H}, 3-\mathrm{OCH}_{3}\right), 4.39\left(\mathrm{~m}, 1 \mathrm{H}, 16 \mathrm{a}-\mathrm{H}_{2}\right), 4.64\left(\mathrm{~m}, 1 \mathrm{H}, 16 \mathrm{a}-\mathrm{H}_{2}\right), 6.63(\mathrm{~s}, 1 \mathrm{H}, 4-\mathrm{H}), 6.71(\mathrm{~d}, 1 \mathrm{H}$, $J=8.5 \mathrm{~Hz}, 2-\mathrm{H}), 7.21(\mathrm{~d}, 1 \mathrm{H}, J=8.5 \mathrm{~Hz}, 1-\mathrm{H}), 7.77\left(\mathrm{~s}, 1 \mathrm{H}, 5^{\prime}-\mathrm{H}\right) .{ }^{13} \mathrm{C} \mathrm{NMR}\left(\delta, \mathrm{ppm}, \mathrm{CDCl}_{3}\right)$ : 11.9 (C-18), 26.0, 28.0, 28.9, 31.3, 31.9, 33.8 (C-13), 38.9, 41.9, 43.5, 46.4 (4'- $\left.{ }^{\prime} \mathrm{H}_{2}\right), 46.9,51.0$ (C-16a), $55.2\left(3-\mathrm{OCH}_{3}\right), 78.6$ (C-17), 111.5 (C-2), 113.8 (C-4), 123.4 (C-5'), 126.3 (C-1), 132.5 (C-10), 137.8 (C-5), 157.4 (C-3).

2.3.25. 3-Benzyloxy-16ß-(4'-cyclopropyl-1 'H-1 ',2',3'-triazol-1'-yl)methylestra-1,3,5(10)-trien$17 \beta$-ol (25a)

Compound 17 (420 mg, $1 \mathrm{mmol}$ ) and cyclopropylacetylene ( $2 \mathrm{mmol}, 0.22 \mathrm{ml}$ ) were used for the synthesis as described in Section 2.3. The crude product was chromatographed on silica gel with 
ethyl acetate $/ \mathrm{CH}_{2} \mathrm{Cl}_{2}(1: 99 \mathrm{v} / \mathrm{v})$ to yield pure $25 \mathrm{a}$ (394 $\left.\mathrm{mg}, 84 \%\right)$ as a white solid. Mp: $278-280$ ${ }^{\circ} \mathrm{C} ; R_{\mathrm{f}}=0.35$ (ss B). (Found $\mathrm{C}, 77.16 ; \mathrm{H}, 7.62 . \mathrm{C}_{31} \mathrm{H}_{37} \mathrm{~N}_{3} \mathrm{O}_{2}$ (483.64) requires $\mathrm{C}, 76.98 ; \mathrm{H}$, 7.71\%). ${ }^{1} \mathrm{H}$ NMR ( $\delta$, ppm, $\left.\mathrm{CDCl}_{3}\right): 0.80$ (s, $\left.3 \mathrm{H}, 18-\mathrm{H}_{3}\right), 0.86$ and $0.97(2 \mathrm{x} \mathrm{m}, 2$ x $2 \mathrm{H}, 2$ "- and 3"H), $2.83\left(\mathrm{~m}, 2 \mathrm{H}, 6-\mathrm{H}_{2}\right), 3.93(\mathrm{~d}, J=9.5 \mathrm{~Hz}, 1 \mathrm{H}, 17-\mathrm{H}), 4.21\left(\mathrm{~m}, 1 \mathrm{H}, 16 \mathrm{a}-\mathrm{H}_{2}\right), 4.64(\mathrm{~m}, 1 \mathrm{H}, 16 \mathrm{a}-$ $\left.\mathrm{H}_{2}\right), 5.03$ (s, 2H, Bn-H $), 6.71(\mathrm{~s}, 1 \mathrm{H}, 4-\mathrm{H}), 6.78(\mathrm{~d}, 1 \mathrm{H}, J=8.5 \mathrm{~Hz}, 2-\mathrm{H}), 7.20(\mathrm{~d}, 1 \mathrm{H}, J=8.5 \mathrm{~Hz}$, 1-H), 7.31 (t, 1H, $\left.J=7.0 \mathrm{~Hz}, 4^{\prime}-\mathrm{H}\right), 7.38$ (t, $2 \mathrm{H}, J=7.0 \mathrm{~Hz}, 3^{\prime}-$ and 5'-H), 7.43 (d, 2H, $J=7.0$ $\mathrm{Hz}, 2^{\prime}-$ and 6'-H). ${ }^{13} \mathrm{C}$ NMR ( $\delta$, ppm, $\mathrm{CDCl}_{3}$ ): 7.8 (C-2" and -3"), 12.3 (C-18), 26.2, 27.4, 29.7,

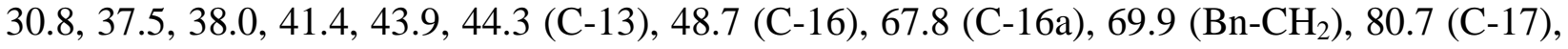
112.3 (C-2), 114.8 (C-4), 126.3 (C-1), 127.4 (C-2' and -6'), 127.8 (C-4'), 128.5 (C-3' and C-5'), 132.7 (C-10), 137.3 (C-1'), 137.8 (C-5), 156.8 (C-3).

2.3.26. 3-Benzyloxy-16ß-(4'-cyclopentyl-1 'H-1 ',2',3'-triazol-1'-yl)methylestra-1,3,5(10)-trien$17 \beta-o l(25 b)$

Compound 17 (420 mg, $1 \mathrm{mmol}$ ) and cyclopentylacetylene $(2 \mathrm{~mol}, 0.22 \mathrm{ml})$ were used for the synthesis as described in Section 2.3. The crude product was chromatographed on silica gel with ethyl acetate/ $\mathrm{CH}_{2} \mathrm{Cl}_{2}(1: 99 \mathrm{v} / \mathrm{v})$ to yield pure $25 \mathrm{~b}$ (350 mg, $\left.68 \%\right)$ as a white solid. Mp: $288-290$ ${ }^{\circ} \mathrm{C} ; R_{\mathrm{f}}=0.38$ (ss B). Found C, 77.58; H, 7.92. $\mathrm{C}_{33} \mathrm{H}_{41} \mathrm{~N}_{3} \mathrm{O}_{2}(511.70)$ requires $\mathrm{C}, 77.46 ; \mathrm{H}, 8.08 \%$ ). ${ }^{1} \mathrm{H}$ NMR ( $\delta$, ppm, $\left.\mathrm{CDCl}_{3}\right): 0.79$ (s, 3H, 18- $\left.\mathrm{H}_{3}\right), 2.75(\mathrm{~s}, 1 \mathrm{H}, 1$ '- $\mathrm{H}), 2.83\left(\mathrm{~m}, 2 \mathrm{H}, 6-\mathrm{H}_{2}\right), 3.94(\mathrm{~d}$, $1 \mathrm{H}, J=9.5 \mathrm{~Hz}, 17-\mathrm{H}), 4.24\left(\mathrm{~m}, 1 \mathrm{H}, 16-\mathrm{H}_{2}\right), 4.67\left(\mathrm{~m}, 1 \mathrm{H}, 16-\mathrm{H}_{2}\right), 5.03$ (s., $\left.2 \mathrm{H}, \mathrm{Bn}-\mathrm{H}_{2}\right), 6.71(\mathrm{~s}$, $1 \mathrm{H}, 4-\mathrm{H}), 6.78(\mathrm{~d}, 1 \mathrm{H}, J=8.5 \mathrm{~Hz}, 2-\mathrm{H}), 7.19$ (d, 1H, $J=8.5 \mathrm{~Hz}, 1-\mathrm{H}), 7.31$ (t, 1H, $J=7.5 \mathrm{~Hz}, 4^{\prime}-$ H), $7.38\left(\mathrm{t}, 2 \mathrm{H}, J=7.5 \mathrm{~Hz}, 3^{\prime}-\right.$ and $\left.5^{\prime}-\mathrm{H}\right), 7.42\left(\mathrm{~d}, 2 \mathrm{H}, J=7.5 \mathrm{~Hz}, 2^{\prime}-\right.$ and $\left.6{ }^{\prime}-\mathrm{H}\right) .{ }^{13} \mathrm{C}$ NMR $(\delta$, ppm, $\mathrm{CDCl}_{3}$ ): 12.3 (C-18), 25.1 (C-3" and -4"), 26.2, 27.5, 29.7, 30.8, 34.3 (C-2" and -5"), 37.5,

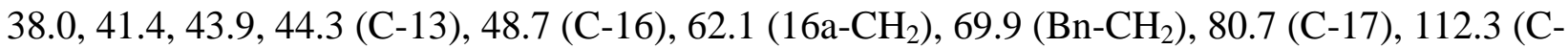
2), 114.8 (C-4), 126.3 (C-1), 127.4 (C-2' and -6'), 127.8 (C-4'), 128.5 (C-3' and -5'), 132.7 (C10), 137.3 (C-1'), 137.8 (C-5), 156.8 (C-3).

\subsubsection{3-Benzyloxy-16ß-(4'-cyclohexyl-1'H-1',2',3'-triazol-1'-yl)methylestra-1,3,5(10)-trien- $17 \beta-o l(25 c)$}

Compound 17 (420 mg, $1 \mathrm{mmol}$ ) and cyclohexylacetylene ( $2 \mathrm{mmol}, 0.22 \mathrm{ml})$ were used for the synthesis as described in Section 2.3. The crude product was chromatographed on silica gel with ethyl acetate/ $\mathrm{CH}_{2} \mathrm{Cl}_{2}(1: 99, \mathrm{v} / \mathrm{v})$ to yield pure $25 \mathrm{c}$ (146 mg, 28\%) as a white solid. Mp: 214-216 
${ }^{\circ} \mathrm{C} ; R_{\mathrm{f}}=0.38$ (ss B). (Found $\mathrm{C}, 77.43 ; \mathrm{H}, 8.36 . \mathrm{C}_{34} \mathrm{H}_{43} \mathrm{~N}_{3} \mathrm{O}_{2}(525.72$ ) requires $\mathrm{C}, 77.68 ; \mathrm{H}, 8.24 \%$ ). ${ }^{1} \mathrm{H}$ NMR ( $\left.\delta, \mathrm{ppm}, \mathrm{CDCl}_{3}\right): 0.79$ (s, 3H, 18- $\left.\mathrm{H}_{3}\right), 2.79$ (m, 4H, 3"- and 5"-H), $3.94(\mathrm{~d}, J=9.5 \mathrm{~Hz}$, 1H, 17-H), $4.25\left(\mathrm{~m}, 1 \mathrm{H}, 16 \mathrm{a}-\mathrm{H}_{2}\right), 4.67\left(\mathrm{~m}, 1 \mathrm{H}, 16 \mathrm{a}-\mathrm{H}_{2}\right), 5.03\left(\mathrm{~s}, 2 \mathrm{H}, \mathrm{Bn}-\mathrm{H}_{2}\right), 6.71(\mathrm{~s}, 1 \mathrm{H}, 4-\mathrm{H})$, $6.78(\mathrm{~d}, 1 \mathrm{H}, \mathrm{J}=8.5 \mathrm{~Hz}, 2-\mathrm{H}), 7.19$ (d, 1H, J = 8.5 Hz, 1-H), 7.32 (d, 1H, $\left.J=7.0 \mathrm{~Hz}, 4^{\prime}-\mathrm{H}\right), 7.38$ (t, $2 \mathrm{H}, J=7.0 \mathrm{~Hz}, 3^{\prime}-$ and $\left.5^{\prime}-\mathrm{H}\right), 7.42\left(\mathrm{~d}, 2 \mathrm{H}, J=7 \mathrm{~Hz}, 2^{\prime}-\right.$ and $\left.6^{\prime}-\mathrm{H}\right) .{ }^{13} \mathrm{C}$ NMR $\left(\delta, \mathrm{ppm}, \mathrm{CDCl}_{3}\right)$ : 12.3 (C-18), 26.0 (C-4"), 26.1 (C-3" and -5”), 26.2, 27.5, 29.7, 30.8 (C-2" and -6"), 33.0 (C-1"), 37.5, 38.0, 41.4, 43.9, 44.3 (C-13), 48.7 (C-16), 62.1 (C-16a), 69.9 (Bn- $\left.\mathrm{CH}_{2}\right), 80.7$ (C-17), 112.3 (C-2), 114.8 (C-4), 126.3 (C-1), 127.4 (C-2' and -6'), 127.8 (C-4'), 128.5 (C-3' and -5'), 132.7 (C-10), 137.3 (C-1'), 137.8 (C-5), 157.8 (C-3).

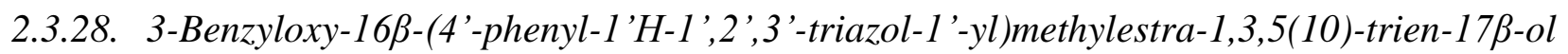
(25d)

Compound $17(420 \mathrm{mg}, 1 \mathrm{mmol})$ and phenylacetylene $(2 \mathrm{mmol}, 0.22 \mathrm{ml})$ were used for the synthesis as described in Section 2.3. The crude product was chromatographed on silica gel with ethyl acetate $/ \mathrm{CH}_{2} \mathrm{Cl}_{2}(5: 95 \mathrm{v} / \mathrm{v})$ to yield pure $25 \mathrm{~d}$ (391 mg, 75\%) as a white solid. Mp: 202-204 ${ }^{\circ} \mathrm{C} ; R_{\mathrm{f}}=0.45$ (ss B). (Found $\mathrm{C}, 78.73 ; \mathrm{H}, 6.98 . \mathrm{C}_{34} \mathrm{H}_{37} \mathrm{~N}_{3} \mathrm{O}_{2}(519.68)$ requires $\mathrm{C}, 78.58 ; \mathrm{H}, 7.18 \%$ ). ${ }^{1} \mathrm{H}$ NMR $\left(\delta, \mathrm{ppm}, \mathrm{C}_{6} \mathrm{D}_{6}\right): 0.68\left(\mathrm{~s}, 3 \mathrm{H}, 18-\mathrm{H}_{3}\right), 2.69\left(\mathrm{~m}, 2 \mathrm{H}, 6-\mathrm{H}_{2}\right), 3.43(\mathrm{dd}, J=9.5 \mathrm{~Hz}, J=4 \mathrm{~Hz}$, 1H, 17-H), 3.77 (dd, 1H, J = 13.5 Hz, J = 7.0 Hz, 16a-H $_{2}, 4.29$ (dd, 1H, J = 13.5 Hz, J = 7.0 Hz, 16a- $\left.\mathrm{H}_{2}\right), 4.83$ (s, 2H, Bn-H $), 6.79$ (s, 1H, 4-H), 6.87 (d, 1H, J = 8.0 Hz, 2-H), 7.02 (s, 1H, 1-H), 7.08 (t, $\left.1 \mathrm{H}, J=7.5 \mathrm{~Hz}, 4^{\prime}-\mathrm{H}\right), 7,26$ (t, $2 \mathrm{H}, J=7.5 \mathrm{~Hz}, 3^{\prime}-$ and $\left.5^{\prime}-\mathrm{H}\right), 7.32$ (d, 2H, $J=7.5 \mathrm{~Hz}, 2^{\prime}-$ and 6'-H), $8.01(\mathrm{~d}, 2 \mathrm{H}, J=7.5 \mathrm{~Hz}, 2$ '- and 6"-H).

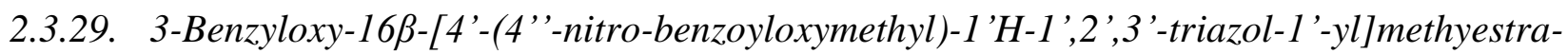
1,3,5(10)-trien-17 $\beta$-ol (25e)

Compound 17 (420 mg, $1 \mathrm{mmol}$ ) and propargyl 4-nitrobenzoate ( $2 \mathrm{mmol}, 210 \mathrm{mg}$ ) were used for the synthesis as described in Section 2.3. The crude product was chromatographed on silica gel ethyl acetate $/ \mathrm{CH}_{2} \mathrm{Cl}_{2}(5: 95 \mathrm{v} / \mathrm{v})$ to yield pure $25 \mathrm{e}(480 \mathrm{mg}, 77 \%)$ as a yellow solid. Mp: 187-189 ${ }^{\circ} \mathrm{C} ; R_{\mathrm{f}}=0.45$ (ss B). (Found C, 69.32; 5.98. $\mathrm{C}_{36} \mathrm{H}_{38} \mathrm{~N}_{4} \mathrm{O}_{6}(622.71$ ) requires C, 69.44; H, 6.15\%). ${ }^{1} \mathrm{H}$ NMR $\left(\delta, \mathrm{ppm}, \mathrm{CDCl}_{3}\right): 0.80\left(\mathrm{~s}, 3 \mathrm{H}, 18-\mathrm{H}_{3}\right), 2.82\left(\mathrm{~m}, 2 \mathrm{H}, 6-\mathrm{H}_{2}\right), 3.94(\mathrm{~d}, J=10.0 \mathrm{~Hz}, 1 \mathrm{H}, 17-$ $\mathrm{H}), 4.32\left(\mathrm{dd}, 1 \mathrm{H}, \mathrm{J}=13.0 \mathrm{~Hz}, \mathrm{~J}=6.0 \mathrm{~Hz}, 16 \mathrm{a}-\mathrm{H}_{2}\right), 4.72\left(\mathrm{t}, 1 \mathrm{H}, \mathrm{J}=6.0 \mathrm{~Hz}, 16 \mathrm{a}-\mathrm{H}_{2}\right), 5.03(\mathrm{~s}, 2 \mathrm{H}$, Bn- $\left.\mathrm{H}_{2}\right), 5.52(\mathrm{~s}, 2 \mathrm{H}$, triazol-H), $6.71(\mathrm{~s}, 1 \mathrm{H}, 4-\mathrm{H}), 6.78(\mathrm{~d}, 1 \mathrm{H}, J=8.5 \mathrm{~Hz}, 2-\mathrm{H}), 7.19(\mathrm{~d}, 1 \mathrm{H}, J=$ 
$8.5 \mathrm{~Hz}, 1-\mathrm{H}), 7.32$ (t, $\left.1 \mathrm{H}, J=7.0 \mathrm{~Hz}, 4^{\prime}-\mathrm{H}\right), 7.38$ (t, $J=7.5 \mathrm{~Hz}, 2 \mathrm{H}, 3^{\prime}{ }^{\prime}-$ and $\left.5^{\prime}-\mathrm{H}\right), 7.42$ (d, $J=$ $7.5 \mathrm{~Hz}, 2 \mathrm{H}, 2$ '- and 6'-H), 8.22 (d, $J=8 \mathrm{~Hz}, 2 \mathrm{H}, 3$ "'- and 5'-H), 8.27 (d, $J=8 \mathrm{~Hz}, 2 \mathrm{H}, 2$ '- and 6"-H). ${ }^{13} \mathrm{C}$ NMR ( $\delta, \mathrm{ppm}, \mathrm{CDCl}_{3}$ ): 12.3 (C-18), 26.2, 27.4, 29.7, 30.8, 37.4, 38.0, 41.2, 43.8, 44.4 (C-13), 48.7 (C-16), 55.5 (C-16a), 58.7 (linker- $\left.\mathrm{CH}_{2}\right), 69.9$ (Bn- $\left.\mathrm{CH}_{2}\right), 80.7$ (C-17), 112.4 (C-2), 114.8 (C-4), 123.5 (C-2' and -6'), 126.3 (C-1), 127.4 (C-2" and -6”), 127.8 (C-4'), 128.5 (C-3" and -5”), 130.9 (C-3' and -5'), 132.5 (C-10), 135.1 (C-1"), 137.3 (C-1'), 137.8 (C-5), 150.7 (C$4 ”), 156.8(\mathrm{C}-3), 164.6(\mathrm{C}=\mathrm{O})$.

2.3.30. 3-Benzyloxy-16ß-(4'-hydroxymethyl-1'H-1',2',3'-triazol-1'-yl)methylestra-1,3,5(10)trien-17 $\beta$-ol (25f)

Compound 25e (210 mg, $0.5 \mathrm{mmol})$ was dissolved in methanol (10 ml) containing $\mathrm{NaOCH}_{3}(14$ $\mathrm{mg}, 0.25 \mathrm{mmol}$ ), and the solution was allowed to stand for $24 \mathrm{~h}$. It was then diluted with water, and the precipitate separating out was filtered off and recrystallized from methanol to afford $\mathbf{2 5 f}$ (232 mg, 98\%) as a white crystalline product. Mp: $283-285{ }^{\circ} \mathrm{C} ; R_{\mathrm{f}}=0.25$ (ss B). (Found C, 73.42; $\mathrm{H}, 7.35 . \mathrm{C}_{29} \mathrm{H}_{35} \mathrm{~N}_{3} \mathrm{O}_{3}(473.61)$ requires $\left.\mathrm{C}, 73.54 ; \mathrm{H}, 7.45 \%\right)$. ${ }^{1} \mathrm{H}$ NMR ( $\delta$, ppm, DMSO-d $\mathrm{d}_{6}$ ): $0.77\left(\mathrm{~s}, 3 \mathrm{H}, 18-\mathrm{H}_{3}\right), 3.77\left(\mathrm{dd}, 1 \mathrm{H}, \mathrm{J}=9.5 \mathrm{~Hz}, \mathrm{~J}=3.5 \mathrm{~Hz}, 16 \mathrm{a}-\mathrm{H}_{2}\right), 4.15(\mathrm{t}, 1 \mathrm{H}, \mathrm{J}=12.5 \mathrm{~Hz}, 16 \mathrm{a}-$ $\left.\mathrm{H}_{2}\right), 5.12(\mathrm{~d}, 1 \mathrm{H}, \mathrm{J}=5.5 \mathrm{~Hz}, 17-\mathrm{H}), 6.68(\mathrm{~s}, 1 \mathrm{H}, 4-\mathrm{H}), 6.74(\mathrm{~d}, 1 \mathrm{H}, J=8.5 \mathrm{~Hz}, 2-\mathrm{H}), 7.16(\mathrm{~d}, J=$ $8.5 \mathrm{~Hz}, 1 \mathrm{H}, 1-\mathrm{H}), 7.31$ (d, 1H, $\left.J=7.0 \mathrm{~Hz}, 4^{\prime}-\mathrm{H}\right), 7.37$ (t, 2H, $J=7.0 \mathrm{~Hz}, 3^{\prime}-$ and 5'-H), 7.41 (d, $2 \mathrm{H}, J=7.0 \mathrm{~Hz}$., $2^{\prime}-$ and $\left.6^{\prime}-\mathrm{H}\right), 7.98\left(\mathrm{~s}, 1 \mathrm{H}\right.$, triazol-H). ${ }^{13} \mathrm{C}$ NMR $\left(\delta, \mathrm{ppm}, \mathrm{DMSO}-\mathrm{d}_{6}\right): 12.3(\mathrm{C}-$ 18), 25.8, 26.9, 29.1, 30.0, 36.9, 37.8, 40.4, 43.4, 43.7 (C-13), 47.8 (C-16a), 55.0 (linker- $\mathrm{CH}_{2}$ ), $68.9\left(\mathrm{Bn}-\mathrm{CH}_{2}\right), 79.5$ (C-17), 112.1 (C-2), 114.4 (C-4), 122.7 (triazol-CH), 126.0 (C-1), 127.4 (C2' and -6'), 127.6 (C-4'), 128.3 (C-3' and -5'), 132.3 (C-10), 137.3 (C-5), 147.6 (triazol-C), 156.0 (C-3).

2.3.31. 3-Benzyloxy-16a-(4'-cyclopropyl-1'H-1',2, '3'-triazol-1'-yl)methylestra-1,3,5(10)-trien$17 \beta$-ol (26a)

Compound 18 (420.0 mg, $1 \mathrm{mmol}$ ) and cyclopropylacetylene ( $2 \mathrm{mmol}, 0.22 \mathrm{ml})$ were used for the synthesis as described in Section 2.3. The crude product was chromatographed on silica gel with ethyl acetate $/ \mathrm{CH}_{2} \mathrm{Cl}_{2}(1: 99 \mathrm{v} / \mathrm{v})$ to yield pure $\mathbf{2 6 a}$ (310 mg, 64\%) as a white solid. Mp: 191-193 ${ }^{\circ} \mathrm{C} ; R_{\mathrm{f}}=0.35$ (ss B). (Found $\mathrm{C}, 76.82 ; \mathrm{H}, 7.94 . \mathrm{C}_{31} \mathrm{H}_{37} \mathrm{~N}_{3} \mathrm{O}_{2}$ (483.64) requires $\mathrm{C}, 76.98 ; \mathrm{H}$, 7.71\%). ${ }^{1} \mathrm{H}$ NMR ( $\left.\delta, p p m, \mathrm{CDCl}_{3}\right): 0.83\left(\mathrm{~s}, 3 \mathrm{H}, 18-\mathrm{H}_{3}\right), 2.83\left(\mathrm{~m}, 2 \mathrm{H}, 6-\mathrm{H}_{2}\right), 3.54(\mathrm{~d}, J=7.5 \mathrm{~Hz}$, 
$1 \mathrm{H}, 17-\mathrm{H}), 4.35\left(\mathrm{dd}, 1 \mathrm{H}, J=13.0 \mathrm{~Hz}, J=7.5 \mathrm{~Hz}, 16 \mathrm{a}-\mathrm{H}_{2}\right), 4.44(\mathrm{dd}, 1 \mathrm{H}, J=13.0 \mathrm{~Hz}, J=7.5 \mathrm{~Hz}$, $\left.16 \mathrm{a}-\mathrm{H}_{2}\right), 5.03\left(\mathrm{~s}, 2 \mathrm{H}, \mathrm{Bn}-\mathrm{H}_{2}\right), 6.71(\mathrm{~s}, 1 \mathrm{H}, 4-\mathrm{H}), 6.77(\mathrm{~d}, 1 \mathrm{H}, J=8.5 \mathrm{~Hz}, 2-\mathrm{H}), 7.19(\mathrm{~d}, 1 \mathrm{H}, J=$ $8.5 \mathrm{~Hz}, 1-\mathrm{H}), 7.31$ (t, 2H, $J=7.5 \mathrm{~Hz}, 4^{\prime}-\mathrm{H}$ and triazol-H), 7.38 (t, 2H, J=7.5 Hz, 3'- and 5'-H),

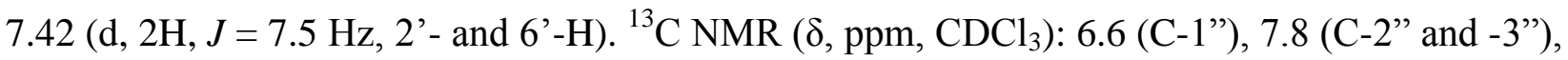
11.8 (C-18), 26.1, 27.2, 28.2, 29.7, 36.6, 38.4, 43.9, 44.3, 44.3 (C-13), 48.3 (C-16), 54.5 (C-16a), $69.9\left(\mathrm{Bn}^{-\mathrm{CH}_{2}}\right), 85.2(\mathrm{C}-17), 112.3(\mathrm{C}-2), 114.8(\mathrm{C}-4), 120.0$ (triazol-CH), $126.3(\mathrm{C}-1), 127.4(\mathrm{C}-$ 2' and -6'), 127.8 (C-4'), 128.5 (C-3' and -5'), 132.6 (C-10), 137.3 (C-1'), 137.8 (C-5), 150.2 (triazol-C), 156.8 (C-3).

2.3.32. 3-Benzyloxy-16a-(4'-cyclopentyl-1'H-1',2',3'-triazol-1'-yl)methylestra-1,3,5(10)-trien$17 \beta-o l(26 b)$

Compound 18 (420 mg, $1 \mathrm{mmol})$ and cyclopentylacetylene $(2 \mathrm{mmol}, 0.22 \mathrm{ml})$ were used for the synthesis as described in Section 2.3. The crude product was chromatographed on silica gel with ethyl acetate $/ \mathrm{CH}_{2} \mathrm{Cl}_{2}(1: 99 \mathrm{v} / \mathrm{v})$ to yield pure $\mathbf{2 6 b}(442 \mathrm{mg}, 86 \%)$ as a white solid. Mp: 268-270 ${ }^{\circ} \mathrm{C} ; R_{\mathrm{f}}=0.36$ (ss B). (Found C, 77.52; H, 7.93. $\mathrm{C}_{33} \mathrm{H}_{41} \mathrm{~N}_{3} \mathrm{O}_{2}(511.70)$ requires $\mathrm{C}, 77.46 ; \mathrm{H}, 8.08 \%$ ).

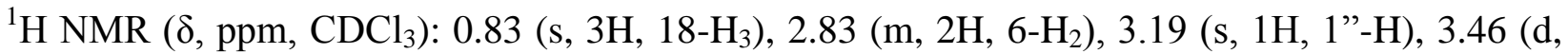
$1 \mathrm{H}, J=7.0 \mathrm{~Hz}, 17-\mathrm{H}), 4.42\left(\mathrm{dd}, 2 \mathrm{H}, J=22.5 \mathrm{~Hz}, J,=6.5 \mathrm{~Hz}, 16-\mathrm{H}_{2}\right), 5.03\left(\mathrm{~s}, 2 \mathrm{H}, \mathrm{Bn}-\mathrm{H}_{2}\right), 6.71$ (s, 1H, 4-H), $6.76(\mathrm{~d}, 1 \mathrm{H}, J=8.5 \mathrm{~Hz}, 2-\mathrm{H}), 7.19(\mathrm{~d}, 1 \mathrm{H}, J=8.5 \mathrm{~Hz}, 1-\mathrm{H}), 7.31$ (t, 1H, $J=7.5 \mathrm{~Hz}$, 4'-H), 7.37 (t, 3H, $J=7.5 \mathrm{~Hz}, 3^{\prime}-, 5^{\prime}-\mathrm{H}$ and triazol-H), 7.42 (d, 2H, $J=7.5 \mathrm{~Hz}, 2^{\prime}-$ and 6'-H). ${ }^{13} \mathrm{C}$ NMR ( $\delta$, ppm, $\mathrm{CDCl}_{3}$ ): 11.9 (C-18), 25.1 (C-3" and -4”), 26.1, 27.2, 28.3, 29.7, 33.2 (C-2" and -5”), 36.6 (2C, C-1”), 36.7, 38.4, 43.9, 44.3 (C-13), 48.4 (C-16), 54.5 (C-16a), 69.9 (Bn$\mathrm{CH}_{2}$ ), 85.2 (C-17), 112.3 (C-2), 114.8 (C-4), 126.3 (C-1), 127.4 (C-3' and -5'), 127.8 (C-4'), 128.5 (C-2' and -6'), 132.6 (C-10), 137.3 (C-1'), 137.8 (C-5), 156.7 (C-3).

2.3.33. 3-Benzyloxy-16a-(4'-cyclohexyl-1 'H-1',2',3'-triazol-1'-yl)methylestra-1,3,5(10)-trien$17 \beta-o l(26 c)$

Compound 18 (420 mg, $1 \mathrm{mmol})$ and cyclohexylacetylene ( $2 \mathrm{mmol}, 0.22 \mathrm{ml})$ were used for the synthesis as described in Section 2.3. The crude product was chromatographed on silica gel with ethyl acetate $/ \mathrm{CH}_{2} \mathrm{Cl}_{2}(2.5: 77.5 \mathrm{v} / \mathrm{v})$ to yield pure 26c $(386 \mathrm{mg}, 76 \%)$ as a white solid. Mp: 261-263 ${ }^{\circ} \mathrm{C} ; R_{\mathrm{f}}=0.34$ (ss B). (Found C, 77.93; H, 8.36. $\mathrm{C}_{34} \mathrm{H}_{43} \mathrm{~N}_{3} \mathrm{O}_{2}(525.72$ ) requires C, 77.68; $\mathrm{H}, 8.24 \%) .{ }^{1} \mathrm{H}$ NMR $\left(\delta, \mathrm{ppm}, \mathrm{CDCl}_{3}\right): 0.83\left(\mathrm{~s}, 3 \mathrm{H}, 18-\mathrm{H}_{3}\right), 2.83\left(\mathrm{~m}, 2 \mathrm{H}, 6-\mathrm{H}_{2}\right), 3.55(\mathrm{~d}, J=7.0$ 
$\mathrm{Hz}, 1 \mathrm{H}, 17-\mathrm{H}), 4.43\left(\mathrm{~m}, 2 \mathrm{H}, 16-\mathrm{H}_{2}\right), 5.03\left(\mathrm{~s}, 2 \mathrm{H}, \mathrm{Bn}-\mathrm{H}_{2}\right), 6.71(\mathrm{~s}, 1 \mathrm{H}, 4-\mathrm{H}), 6.77$ (d, 1H, J = 8.5 Hz, 2-H), 7.19 (d, 1H, J = 8.5 Hz, 1-H), $7.31\left(\mathrm{t}, 2 \mathrm{H}, J=7.0 \mathrm{~Hz}, 4^{\prime}-\mathrm{H}\right.$ and triazol-H), 7.37 (t, 2H, $J=7.0 \mathrm{~Hz} .3^{\prime}-$ and 5'-H), $7.42\left(\mathrm{~d}, 2 \mathrm{H}, J=7 \mathrm{~Hz}, 2^{\prime}-\right.$ and $\left.6{ }^{\prime}-\mathrm{H}\right) .{ }^{13} \mathrm{C}$ NMR $\left(\delta, \mathrm{ppm}, \mathrm{CDCl}_{3}\right): 11.9$ (C-18), 25.9 (C-4”), 26.1 (C-3" and -5”), 27.2, 28.3, 29.7 (C-2" and -6”), 32.9, 33.0, 36.6, 38.4,

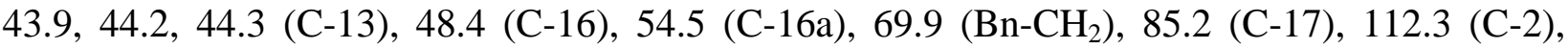
114.8 (C-4), 126.3 (C-1), 127.4 (C-2' and -6'), 127.8 (C-4'), 128.5 (C-3' and -5'), 132.6 (C-10), 137.3 (C-1'), 137.8 (C-5), 156.7 (C-3).

2.3.34. 3-Benzyloxy-16a-(4'-phenyl-1 'H-1',2',3'-triazol-1'-yl)methylestra-1,3,5(10)-trien-17 $\beta$-ol (26d)

Compound 18 (420 mg, $1 \mathrm{mmol})$ and phenylacetylene ( $2 \mathrm{mmol}, 0.22 \mathrm{ml})$ were used for the synthesis as described in Section 2.3. The crude product was chromatographed on silica gel with ethyl acetate $/ \mathrm{CH}_{2} \mathrm{Cl}_{2}$ 5:95 v/v) to yield pure 26d (372 mg, 71\%) as a white solid. Mp: 132-134 ${ }^{\circ} \mathrm{C} ; R_{\mathrm{f}}=0.38$ (ss B). (Found C, 78.63; H, 6.97. $\mathrm{C}_{34} \mathrm{H}_{37} \mathrm{~N}_{3} \mathrm{O}_{2}(519.68)$ requires $\mathrm{C}, 78.58 ; \mathrm{H}, 7.18 \%$ ). ${ }^{1} \mathrm{H}$ NMR $\left(\delta, \mathrm{ppm}, \mathrm{CDCl}_{3}\right): 0.84\left(\mathrm{~s}, 3 \mathrm{H}, 18-\mathrm{H}_{3}\right), 2.83\left(\mathrm{~m}, 2 \mathrm{H}, 6-\mathrm{H}_{2}\right), 3.58(\mathrm{~d}, 1 \mathrm{H}, J=7.5 \mathrm{~Hz}, 17-$ $\mathrm{H}), 4.46\left(\mathrm{dd}, 2 \mathrm{H}, J=13.5 \mathrm{~Hz}, J=8.0 \mathrm{~Hz}, 16 \mathrm{a}-\mathrm{H}_{2}\right), 4.55(\mathrm{dd}, 1 \mathrm{H}, J=13.5 \mathrm{~Hz}, J=8.0 \mathrm{~Hz}, 16 \mathrm{a}-$ H2) $5.03\left(\mathrm{~s}, 2 \mathrm{H}, \mathrm{Bn}-\mathrm{H}_{2}\right), 6.71(\mathrm{~s}, 1 \mathrm{H}, 4-\mathrm{H}), 6.78(\mathrm{~d}, 1 \mathrm{H}, J=8.5 \mathrm{~Hz}, 2-\mathrm{H}), 7.19$ (d, 1H, J = 8.5 Hz, 1-H), 7.30-7.86 (m, 11H, 2'-, 6'-, 3'-, 5'-, 4'-, 2'”-, 6"'-, 3'-, 5'”-, 4"'- and triazol-H). ${ }^{13} \mathrm{C}$ NMR $(\delta$, ppm, $\left.\mathrm{CDCl}_{3}\right)$ : 11.8 (C-18), 26.1, 27.2, 28.2, 29.6, 36.5, 38.4, 43.9, 44.3, 48.3 (C-16), 54.6 (C16a), 62.1, 69.9 (Bn-CH ( $_{2}, 85.2$ (C-17), 112.3 (C-2), 114.8 (C-4), 123.8 (triazol-CH), 125.7 (C-2' and -6'), 126.3 (C-1'), 127.4 (C-2” and -6”), 127.8 (C-4'), 128.2 (C-4), 128.5 (C-3" and -5”), 128.8 (C-3' and -5'), 130.4 (C-10), 132.6 (C-1’), 137.3 (C-1'), 137.8 (C-5), 156.8 (C-3).

2.3.35. 3-Benzyloxy-16a-[4'-(4'”-nitro-benzoyloxymethyl)-1 'H-1',2',3'-triazol-1 '-yl]methylestra1,3,5(10)-trien-17 $\beta$-ol (26e)

Compound 18 (420 mg, $1 \mathrm{mmol}$ ) and propargyl 4-nitrobenzoate (2 mmol, $210 \mathrm{mg}$ ) were used for the synthesis as described in Section 2.3. The crude product was chromatographed on silica gel with ethyl acetate/ $\mathrm{CH}_{2} \mathrm{Cl}_{2}(5: 95 \mathrm{v} / \mathrm{v})$ to yield pure 26e (484 mg, 77\%) as a yellow solid. Mp: 94-96 ${ }^{\circ} \mathrm{C} ; R_{\mathrm{f}}=0.40$ (ss B). (Found C, 69.73; H, 5.94. $\mathrm{C}_{36} \mathrm{H}_{38} \mathrm{~N}_{4} \mathrm{O}_{6}(622.71$ ) requires $\mathrm{C}, 69.44 ; \mathrm{H}$, 6.15\%). ${ }^{1} \mathrm{H}$ NMR $(\delta, p p m$, DMSO-d 6$): 0.70\left(\mathrm{~s}, 3 \mathrm{H}, 18-\mathrm{H}_{3}\right), 3.33\left(\mathrm{~m}, 2 \mathrm{H}, 6-\mathrm{H}_{2}\right), 4.38(\mathrm{dd}, 1 \mathrm{H}, J=$ $\left.13.5 \mathrm{~Hz}, J=9.0 \mathrm{~Hz}, 16 \mathrm{a}-\mathrm{H}_{2}\right), 4.52\left(\mathrm{dd}, 1 \mathrm{H}, J=13.5 \mathrm{~Hz}, J=5.0 \mathrm{~Hz}, 16 \mathrm{a}-\mathrm{H}_{2}\right), 4.86(\mathrm{~d}, 1 \mathrm{H}, J=5$ 
Hz, 17-H), 5.02 (s, 2H, Bn-H2), 5.47 (s, 2H, linker-H $_{2}, 6.64$ (d, 1H, J = $\left.2.0 \mathrm{~Hz}, 4-\mathrm{H}\right), 6.72$ (dd, $1 \mathrm{H}, \mathrm{J}=8.5 \mathrm{~Hz}, J=2.0 \mathrm{~Hz}, 2-\mathrm{H}), 7.10(\mathrm{~d}, 1 \mathrm{H}, J=8.5 \mathrm{~Hz}, 1-\mathrm{H}), 7.31\left(\mathrm{t}, 1 \mathrm{H}, J=7.0 \mathrm{~Hz}, 4^{\prime}-\mathrm{H}\right)$, 7.37 (t, 2H, $J=7.0 \mathrm{~Hz}, 3^{\prime}-$ and 5'-H), 7.42 (d, 2H, $J=7.0 \mathrm{~Hz}, 2^{\prime}-$ and 6'-H), 8.16 (d, 2H, $J=9.0$ $\mathrm{Hz}, 3$ "'- and 5"-H), $8.28\left(\mathrm{~d}, 2 \mathrm{H}, J=9.0 \mathrm{~Hz}, 2\right.$ "- and 6"-H), 8.32 (s, 1H, triazol-H). ${ }^{13} \mathrm{C}$ NMR $(\delta$, ppm, DMSO-d 6 ): 11.7 (C-18), 25.7, 26.6, 27.1, 29.0, 30.6, 36.4, 37.9, 43.4, 43.4 (C-13), 43.7 (C-

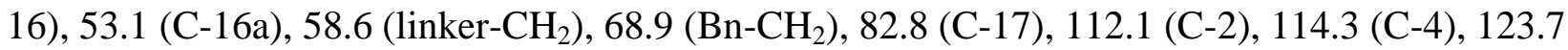
(C-2' and -6'), 125.1 (triazol-CH), 125.9 (C-1), 127.4 (C-2” and -6”), 127.5 (C-4'), 128.3 (C-3” and -5”), 130.6 (C-3' and -5'), 132.1 (C-10), 134.7 (C-1'), 137.2 (C-1'), 137.3 (C-5), 141.1 (triazol-C), 150.1 (C-4”), 155.9 (C-3), 163.9 (C=O).

2.3.36. 3-Benzyloxy-16a-(4'-hydroxymethyl-1 'H-1',2',3 '-triazol-1 '-yl)methylestra-1,3,5(10)trien-17 $\beta$-ol (26f)

Compound 26e (210 mg, $0.5 \mathrm{mmol})$ was dissolved in methanol $(10 \mathrm{ml})$ containing $\mathrm{NaOCH}_{3}(14$ $\mathrm{mg}, 0.25 \mathrm{mmol}$ ), and the solution was allowed to stand for $24 \mathrm{~h}$. It was then diluted with water, and the precipitate separating out was filtered off and recrystallized from a mixture of acetone/hexane to afford $26 \mathbf{f}(190 \mathrm{mg}, 89 \%)$ as a white crystalline product. Mp: $152-154{ }^{\circ} \mathrm{C} ; R_{\mathrm{f}}=$ 0.20 (ss B). (Found C, 73.72; H, 7.63. $\mathrm{C}_{29} \mathrm{H}_{35} \mathrm{~N}_{3} \mathrm{O}_{3}$ (473.61) requires $\mathrm{C}, 73.54 ; \mathrm{H}, 7.45 \%$ ). ${ }^{1} \mathrm{H}$ NMR ( $\delta$, ppm, DMSO-d $\left.)_{6}\right): 0.71\left(\mathrm{~s}, 3 \mathrm{H}, 18-\mathrm{H}_{3}\right), 2.73\left(\mathrm{~m}, 2 \mathrm{H}, 6 \mathrm{H}_{2}\right), 3.29(\mathrm{~d}, J=8.0 \mathrm{~Hz}, 1 \mathrm{H}, 17-$ H), $4.28\left(\mathrm{dd}, 2 \mathrm{H}, J=13.0 \mathrm{~Hz}, J=10.0 \mathrm{~Hz}, 16 \mathrm{a}-\mathrm{H}_{2}\right), 4.47(\mathrm{dd}, 1 \mathrm{H}, J=13.0 \mathrm{~Hz}, J=4.5 \mathrm{~Hz}, 16 \mathrm{a}-$ $\left.\mathrm{H}_{2}\right), 4.51\left(\mathrm{~s}, 2 \mathrm{H}, \mathrm{Bn}-\mathrm{H}_{2}\right), 4.87\left(\mathrm{~s}, 1 \mathrm{H}\right.$, linker- $\left.\mathrm{H}_{2}\right), 5.03\left(\mathrm{~s}, 2 \mathrm{H}\right.$, triazol- $\left.\mathrm{H}_{2}\right), 5.15\left(\mathrm{~s}, 1 \mathrm{H}\right.$, linker- $\left.\mathrm{H}_{2}\right)$, $6.68(\mathrm{~s}, 1 \mathrm{H}, 4-\mathrm{H}), 6.74(\mathrm{~d}, 1 \mathrm{H}, J=8.5 \mathrm{~Hz}, 2-\mathrm{H}), 7.15(\mathrm{~d}, 1 \mathrm{H}, J=8.5 \mathrm{~Hz}, 1-\mathrm{H}), 7.31$ (t, $1 \mathrm{H}, J=7.0$ $\left.\mathrm{Hz}, 4^{\prime}-\mathrm{H}\right), 7.37$ (t, 2H, J = 7.0 Hz, 3'- and 5'-H), 7.41 (d, 2H, J=7.0 Hz, 2'- and 6'-H), 7.97 (s, $1 \mathrm{H}$, triazol-H). ${ }^{13} \mathrm{C}$ NMR $(\delta$, ppm, DMSO-d 6 ): 11.8 (C-18), 25.8, 26.7, 27.3, 29.1, 36.4, 38.1,

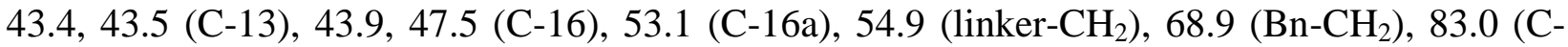
17), 112.1 (C-2), 114.4 (C-4), 122.7 (triazol-CH), 126.0 (C-1), 127.4 (C-2' and -6'), 127.6 (C-4'), 128.3 (C-3' and -5'), 132.3 (C-10), 137.3 (C-1'), 137.4 (C-5), 147.6 (triazol-C), 156.0 (C-3).

2.3.37. 3-Benzyloxy-16ß-(4'-cyclopropyl-1'H-1',2',3'-triazol-1'-yl)methylestra-1,3,5(10)-trien17a-ol (27a)

Compound 19 (420.0 mg, $1 \mathrm{mmol})$ and cyclopropylacetylene ( $2 \mathrm{mmol}, 0.22 \mathrm{ml})$ were used for the synthesis as described in Section 2.3. The crude product was chromatographed on silica gel with 
ethyl acetate $/ \mathrm{CH}_{2} \mathrm{Cl}_{2}(5: 95 \mathrm{v} / \mathrm{v})$ to yield pure $27 \mathbf{a}(454 \mathrm{mg}, 93 \%)$ as white crystals. Mp: 199-201 ${ }^{\circ} \mathrm{C} ; R_{\mathrm{f}}=0.38$ (ss B). (Found $\mathrm{C}, 77.15 ; \mathrm{H}, 7.62 . \mathrm{C}_{31} \mathrm{H}_{37} \mathrm{~N}_{3} \mathrm{O}_{2}$ (483.64) requires C, 76.98; H, 7.71\%). ' $\mathrm{H} \mathrm{NMR}\left(\delta, \mathrm{ppm}, \mathrm{CDCl}_{3}\right): 0.77$ (s, 3H, 18- $\left.\mathrm{H}_{3}\right), 0.87$ and 0.98 (2 x s, 2 x 2H, 2"- and 3"'$\left.\mathrm{H}_{2}\right), 2.05$ (s, 1H, 1"'-H), $2.84\left(\mathrm{~m}, 2 \mathrm{H}, 6-\mathrm{H}_{2}\right), 3.66(\mathrm{~s}, 1 \mathrm{H}, 17-\mathrm{H}), 4.42$ (m, 2H, 16a- $\left.\mathrm{H}_{2}\right), 5.03$ (s, 2H, Bn-H $\left.{ }_{2}\right), 6.71(\mathrm{~s}, 1 \mathrm{H}, 4-\mathrm{H}), 6.78$ (d, 1H, $\left.J=8.5 \mathrm{~Hz}, 2-\mathrm{H}\right), 7.21(\mathrm{~d}, 1 \mathrm{H}, J=8.5 \mathrm{~Hz}, 1-\mathrm{H}), 7.31$ (t, 1H, $\left.J=7.0 \mathrm{~Hz}, 4^{\prime}-\mathrm{H}\right), 7.38$ (t, 2H, $J=7.0 \mathrm{~Hz}, 3^{\prime}$ - and 5'-H), 7.43 (d, 2H, $J=7.0 \mathrm{~Hz}, 2^{\prime}$ - and 6 '-H). ${ }^{13} \mathrm{C}$ NMR ( $\left.\delta, \mathrm{ppm}, \mathrm{CDCl}_{3}\right): 6.7$ (C-1"), 7.7 (C-2” and -3”), 17.9 (C-18), 25.9, 27.9, 29.7,

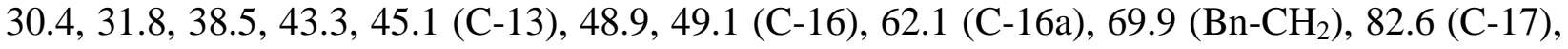
112.3 (C-2), 114.8 (C-4), 126.3 (C-1), 127.4 (C-2' and -6'), 127.8 (C-4'), 128.5 (C-3' and -5'), 132.7 (C-10), 137.3 (C-1'), 137.9 (C-5), 156.7 (C-3).

2.3.38. 3-Benzyloxy-16 3 -(4'-cyclopentyl-1 'H-1',2',3'-triazol-1'-yl)methylestra-1,3,5(10)-trien$17 a-o l(27 b)$

Compound 19 (420 mg, $1 \mathrm{mmol})$ and cyclopentylacetylene $(2 \mathrm{mmol}, 0.22 \mathrm{ml})$ were used for the synthesis as described in Section 2.3. The crude product was chromatographed on silica gel with ethyl acetate $/ \mathrm{CH}_{2} \mathrm{Cl}_{2}(5: 95 \mathrm{v} / \mathrm{v})$ to yield pure $\mathbf{2 7 b}(408 \mathrm{mg}, 79 \%)$ as white crystalline. Mp: 220-222 ${ }^{\circ} \mathrm{C} ; R_{\mathrm{f}}=0.40$ (ss B). (Found C, 77.32; H, 7.93. $\mathrm{C}_{33} \mathrm{H}_{41} \mathrm{~N}_{3} \mathrm{O}_{2}$ (511.70) requires C, 77.46; $\mathrm{H}, 8.08 \%) .{ }^{1} \mathrm{H}$ NMR $\left(\delta, \mathrm{ppm}, \mathrm{CDCl}_{3}\right): 0.76$ (s, 3H, 18- $\left.\mathrm{H}_{3}\right), 2.84\left(\mathrm{~m}, 2 \mathrm{H}, 6-\mathrm{H}_{2}\right), 3.20$ (s, 1H, 1"H), $3.67(\mathrm{~s}, 1 \mathrm{H}, 17-\mathrm{H}), 4.43\left(\mathrm{~m}, 2 \mathrm{H}, 16 \mathrm{a}-\mathrm{H}_{2}\right), 5.03\left(\mathrm{~s}, 2 \mathrm{H}, \mathrm{Bn}-\mathrm{H}_{2}\right), 6.72(\mathrm{~s}, 1 \mathrm{H}, 4-\mathrm{H}), 6.78$ (dd, $1 \mathrm{H}, J=8.5 \mathrm{~Hz}, J=2.0 \mathrm{~Hz}, 2-\mathrm{H}), 7.21(\mathrm{~d}, 1 \mathrm{H}, J=8.5 \mathrm{~Hz}, 1-\mathrm{H}), 7.31$ (t, 1H, $\left.J=7.0 \mathrm{~Hz}, 4^{\prime}-\mathrm{H}\right)$, $7.38\left(\mathrm{t}, 3 \mathrm{H}, J=7.0 \mathrm{~Hz}, 3^{\prime}\right.$ - and 5'-H, triazol-H), $7.43\left(\mathrm{~d}, 2 \mathrm{H}, J=7.0 \mathrm{~Hz}, 2^{\prime}-\right.$ and $\left.6{ }^{\prime}-\mathrm{H}\right) .{ }^{13} \mathrm{C}$ NMR ( $\delta$, ppm, $\mathrm{CDCl}_{3}$ ): 18.0 (C-18), 25.1 (C-3" and -5"), 25.9, 28.0, 29.7, 30.4, 31.8 (C-2" and -6"), 33.2, 36.7, 38.5, 43.3, 45.1 (C-13), 48.9 (C-16), 49.1 (C-1”), 54.3 (C-16a), 69.9 (Bn-CH $), 82.6$ (C-17), 112.3 (C-2), 114.8 (C-4), 126.3 (C-1), 127.4 (C-2' and -6'), 127.8 (C-4'), 128.5 (C-3' and -5’), 132.7 (C-10), 137.3 (C-1'), 137.9 (C-5), 156.7 (C-3).

2.3.39. 3-Benzyloxy-16ß-(4'-cyclohexyl-1 'H-1',2',3'-triazol-1'-yl)methylestra-1,3,5(10)-trien$17 a-o l(27 c)$

Compound 19 (420 mg, $1 \mathrm{mmol})$ and cyclohexylacetylene $(2 \mathrm{mmol}, 0.22 \mathrm{ml})$ were used for the synthesis as described in Section 2.3. The crude product was chromatographed on silica gel with ethyl acetate $/ \mathrm{CH}_{2} \mathrm{Cl}_{2}(5: 95 \mathrm{v} / \mathrm{v})$ to yield pure $27 \mathrm{c}(360 \mathrm{mg}, 68 \%)$ as white crystalline product. 
Mp: $243-245{ }^{\circ} \mathrm{C} ; R_{\mathrm{f}}=0.38$ (ss B). (Found C, 77.54; H, 8.38. $\mathrm{C}_{34} \mathrm{H}_{43} \mathrm{~N}_{3} \mathrm{O}_{2}$ (525.72) requires $\mathrm{C}$, 77.68; H, 8.24\%). ${ }^{1} \mathrm{H}$ NMR ( $\delta$, ppm, $\left.\mathrm{CDCl}_{3}\right): 0.75$ (s, 3H, 18- $\left.\mathrm{H}_{3}\right), 2.84\left(\mathrm{~m}, 2 \mathrm{H}, 6-\mathrm{H}_{2}\right), 3.68$ (s, $1 \mathrm{H}, 17-\mathrm{H}), 4.44\left(\mathrm{~m}, 2 \mathrm{H}, 16 \mathrm{a}-\mathrm{H}_{2}\right), 5.03\left(\mathrm{~s}, 2 \mathrm{H}, \mathrm{Bn}-\mathrm{H}_{2}\right), 6.72(\mathrm{~s}, 1 \mathrm{H}, 4-\mathrm{H}), 6.78$ (d, 1H, J = $8.5 \mathrm{~Hz}$, 2-H), $7.21(\mathrm{~d}, 1 \mathrm{H}, J=8.5 \mathrm{~Hz}, 1-\mathrm{H}), 7.32\left(\mathrm{t}, 1 \mathrm{H}, J=7.0 \mathrm{~Hz}, 4^{\prime}-\mathrm{H}\right), 7.38$ (t, $3 \mathrm{H}, J=7.0 \mathrm{~Hz}, 3^{\prime}-$ and $5^{\prime}-\mathrm{H}$, triazol-H), $7.43\left(\mathrm{~d}, 2 \mathrm{H}, J=7.0 \mathrm{~Hz}, 2^{\prime}-\right.$ and $\left.6{ }^{\prime}-\mathrm{H}\right) .{ }^{13} \mathrm{C}$ NMR ( $\left.\delta, \mathrm{ppm}, \mathrm{CDCl}_{3}\right): 17.9(\mathrm{C}-18)$, 25.9 (C-4"), 26.0, 26.1 (C-3" and -5"), 27.9, 29.7, 30.4, 31.8 (C-2" and -6"), 32.1, 32.9 (C-1"), 38.5, 43.3, 45.1 (C-13), 48.9, 49.1 (C-16), 62.1 (C-16a), 69.9 (Bn- $\left.\mathrm{CH}_{2}\right), 82.5$ (C-17), 112.3 (C-2), 114.7 (C-4), 126.3 (C-1), 127.4 (C-2' and -6'), 127.8 (C-4'), 128.5 (C-3' and -5'), 132.7 (C-10), 137.2 (C-1'), 137.9 (C-5), 156.7 (C-3).

2.3.40. 3-Benzyloxy-16ß-(4'-phenyl-1'H-1',2',3'-triazol-1'-yl)methylestra-1,3,5(10)-trien-17a-ol (27d)

Compound 19 (420 mg, $1 \mathrm{mmol})$ and phenylacetylene $(2 \mathrm{mmol}, 0.22 \mathrm{ml})$ were used for the synthesis as described in Section 2.3. The crude product was chromatographed on silica gel with ethyl acetate $/ \mathrm{CH}_{2} \mathrm{Cl}_{2}(10: 90 \mathrm{v} / \mathrm{v})$ to yield pure $27 \mathbf{d}(487 \mathrm{mg}, 93 \%)$ as white crystals. Mp: 202-204 ${ }^{\circ} \mathrm{C} ; R_{\mathrm{f}}=0.45$ (ss B). (Found C, 78.68; H, 7.38. $\mathrm{C}_{34} \mathrm{H}_{37} \mathrm{~N}_{3} \mathrm{O}_{2}(519.68$ ) requires C, 78.58; $\mathrm{H}, 7.18 \%) .{ }^{1} \mathrm{H}$ NMR ( $\delta$, ppm, $\left.\mathrm{CDCl}_{3}\right): 0.79$ (s, 3H, 18- $\left.\mathrm{H}_{3}\right), 2.84$ (m, 2H, 6- $\left.\mathrm{H}_{2}\right), 3.72$ (s, 1H, 17H), $4.48\left(\mathrm{dd}, 1 \mathrm{H}, J=13.5 \mathrm{~Hz}, J=7.5 \mathrm{~Hz}, 16 \mathrm{a}-\mathrm{H}_{2}\right), 4.56\left(\mathrm{t}, 1 \mathrm{H}, J=13.5 \mathrm{~Hz}, 16 \mathrm{a}-\mathrm{H}_{2}\right), 5.03(\mathrm{~s}, 2 \mathrm{H}$, Bn- $\left.\mathrm{H}_{2}\right), 6.72(\mathrm{~s}, 1 \mathrm{H}, 4-\mathrm{H}), 6.78(\mathrm{~d}, 1 \mathrm{H}, J=8.5 \mathrm{~Hz}, 2-\mathrm{H}), 7.21$ (d, 1H, $\left.J=8.5 \mathrm{~Hz}, 1-\mathrm{H}\right), 7.33$ (t, $\left.1 \mathrm{H}, J=7.5 \mathrm{~Hz}, 4^{\prime}-\mathrm{H}\right), 7.38$ (t, 2H, $J=7.5 \mathrm{~Hz}, 3^{\prime}-$ and 5'-H), 7.42 (d, $J=3.5 \mathrm{~Hz}, 4 \mathrm{H}, 2^{\prime}$ '- and 6'$\mathrm{H}, 3$ "- and 5"-H), $7.84\left(\mathrm{~d}, 2 \mathrm{H}, J=7.5 \mathrm{~Hz}, 2\right.$ "- and 6"-H), $7.88\left(\mathrm{~s}, 1 \mathrm{H}\right.$, triazol-H). ${ }^{13} \mathrm{C}$ NMR $(\delta$, ppm, $\mathrm{CDCl}_{3}$ ): 17.9 (C-18), 25.9, 27.9, 29.7, 30.4, 31.8, 38.5, 43.3, 45.2 (C-13), 48.9, 49.1 (C-16), 54.6 (C-16a), $69.9\left(\mathrm{Bn}-\mathrm{CH}_{2}\right), 82.6$ (C-17), 112.3 (C-2), 114.8 (C-4), 119.6 (triazol-CH), 125.7 (C-2' and -6'), 126.3 (C-1'), 127.4 (C-2" and -6"), 127.8 (C-4'), 128.2 (C-4"), 128.5 (C-3" and 5”), 128.8 (C-3' and -5'), 130.5 (C-10), 132.64 (C-1”), 137.3 (C-1'), 137.9 (C-5), 147.7 (triazolC); 156.8 (C-3).

2.3.41. 3-Benzyloxy-163-[4'-(4'"-nitro-benzoyloxymethyl)-1'H-1',2',3'-triazol-1'-yl]methylestra1,3,5(10)-trien-17a-ol (27e) 
Compound 19 (420.0 mg, $1 \mathrm{mmol})$ and propargyl 4-nitrobenzoate (2 mmol, $210 \mathrm{mg}$ ) were used for the synthesis as described in Section 2.3. The crude product was chromatographed on silica gel with ethyl acetate/ $\mathrm{CH}_{2} \mathrm{Cl}_{2}(10: 90 \mathrm{v} / \mathrm{v})$ to yield pure $27 \mathrm{e}(550 \mathrm{mg}, 88 \%)$ as yellow crystals. Mp: $177-179{ }^{\circ} \mathrm{C} ; R_{\mathrm{f}}=0.48$ (ss B). (Found C, 69.55; H, 5.93. $\mathrm{C}_{36} \mathrm{H}_{38} \mathrm{~N}_{4} \mathrm{O}_{6}$ (622.71) requires: C, 69.44; H, 6.15\%). ${ }^{1} \mathrm{H}$ NMR ( $\delta$, ppm, DMSO-d 6 ): 0.65 (s, 3H, 18- $\left.\mathrm{H}_{3}\right), 2.73\left(\mathrm{~m}, 2 \mathrm{H}, 6-\mathrm{H}_{2}\right), 4.40$ $\left(\mathrm{dd}, 1 \mathrm{H}, J=13.0 \mathrm{~Hz}, J=8.5 \mathrm{~Hz}, 16 \mathrm{a}-\mathrm{H}_{2}\right), 4.56\left(\mathrm{dd}, 1 \mathrm{H}, J=13.5 \mathrm{~Hz}, J=7.5 \mathrm{~Hz}, 16 \mathrm{a}-\mathrm{H}_{2}\right), 4.63$ $(\mathrm{d}, 1 \mathrm{H}, J=5.0 \mathrm{~Hz}, 17-\mathrm{H}), 5.04\left(\mathrm{~s}, 2 \mathrm{H}, \mathrm{Bn}-\mathrm{H}_{2}\right), 5.47$ (s, 2H, triazol-H $), 6.68(\mathrm{~s}, 1 \mathrm{H}, 4-\mathrm{H}), 6.74(\mathrm{~d}$, $1 \mathrm{H}, J=8.5 \mathrm{~Hz}, 2-\mathrm{H}), 7.16(\mathrm{~d}, 1 \mathrm{H}, J=8.5 \mathrm{~Hz}, 1-\mathrm{H}), 7.31\left(\mathrm{t}, 1 \mathrm{H}, J=7.0 \mathrm{~Hz}, 4^{\prime}-\mathrm{H}\right), 7.37$ (t, $2 \mathrm{H}, J$ $=7.0 \mathrm{~Hz}, 3^{\prime}{ }^{\prime}$ and 5'-H), $7.41\left(\mathrm{~d}, 2 \mathrm{H}, J=7.0 \mathrm{~Hz}, 2^{\prime}{ }^{\prime}-\right.$ and 6'-H), $8.18(\mathrm{~d}, 2 \mathrm{H}, J=8.5 \mathrm{~Hz}, 3$ ''- and $5 "-\mathrm{H}), 8.33$ (d, 3H, $J=6 \mathrm{~Hz}, 2$ "- and 6"-H, triazol-H). ${ }^{13} \mathrm{C}$ NMR ( $\delta$, ppm, DMSO-d 6 ): $17.5(\mathrm{C}-$ 18), 25.6, 27.5, 29.2, 29.6, 31.8, 38.2, 42.9, 44.5 (C-13), 48.2, 49.1 (C-16), 53.6 (C-16a), 58.7 (linker- $\mathrm{CH}_{2}$ ), 68.9 (Bn-CH$), 80.8$ (C-17), 112.1 (C-2), 114.4 (C-4), 123.8 (C-2' and C-6'), 125.0 (triazol-CH), 126.1 (C-1), 127.4 (C-2” and -6”), 127.6 (C-4'), 128.3 (C-3” and -5”), 130.6 (C-3' and -5'), 132.3 (C-10), 134.7 (C-1'), 137.3 (C-5 and C-1'), 141.1 (triazol-C), 150.2 (C-4”), 160.0 (C-3), $163.9(\mathrm{C}=\mathrm{O})$.

2.3.42. 3-Benzyloxy-16ß-(4'-hydroxymethyl-1'H-1',2',3'-triazol-1'-yl)methylestra-1,3,5(10)trien-17a-ol (27f)

Compound 27e $(210 \mathrm{mg}, 0.5 \mathrm{mmol})$ was dissolved in methanol $(10 \mathrm{ml})$ containing $\mathrm{NaOCH}_{3}(14$ $\mathrm{mg}, 0.25 \mathrm{mmol}$ ), and the solution was allowed to stand for $24 \mathrm{~h}$. It was then diluted with water, and the precipitate separating out was filtered off and recrystallized from methanol to afford 27e (273 mg, 99\%) as a white crystalline product. Mp: $172-174{ }^{\circ} \mathrm{C} ; R_{\mathrm{f}}=0.25$ (ss B). (Found C, 73.68; $\mathrm{H}, 7.66 . \mathrm{C}_{29} \mathrm{H}_{35} \mathrm{~N}_{3} \mathrm{O}_{3}$ (473.61) requires $\left.\mathrm{C}, 73.54 ; \mathrm{H}, 7.45 \%\right)$. ${ }^{1} \mathrm{H}$ NMR ( $\delta$, ppm, DMSO-d 6 ): $0.67\left(\mathrm{~s}, 3 \mathrm{H}, 18-\mathrm{H}_{3}\right), 2.74\left(\mathrm{~m}, 2 \mathrm{H}, 6-\mathrm{H}_{2}\right), 3.43(\mathrm{~s}, 1 \mathrm{H}, 17-\mathrm{H}), 4.34\left(\mathrm{~m}, 1 \mathrm{H}, 16 \mathrm{a}-\mathrm{H}_{2}\right), 4.50(\mathrm{~m}, 3 \mathrm{H}$, $16 \mathrm{a}-\mathrm{H}_{2}$ and $\left.\mathrm{Bn}-\mathrm{H} 2\right), 4.61$ (brs, 1H, OH), 5.04 (s, 2H, triazol- $\left.\mathrm{H}_{2}\right), 5.16$ (brs, 1H, OH), 6.69 (s, 1H, 4-H), 6.74 (d, 1H, J=8.5 Hz, 2-H), 7.17 (d, 1H, J = 8.5 Hz, 1-H), 7.31 (d, 1H, J = 7.0 Hz, 4'-H), 7.37 (t, 2H, $J=7.0 \mathrm{~Hz}, 3^{\prime}$ - and 5'-H), 7.41 (d, 2H, J = 7.0 Hz, 2'- and 6'-H), 8.00 (s, 1H, triazolH). ${ }^{13} \mathrm{C}$ NMR $\left(\delta\right.$, ppm, DMSO-d $\left.\mathrm{d}_{6}\right): 17.5(\mathrm{C}-18), 25.6,27.5,29.2,29.6,31.9,38.2,43.0,44.5(\mathrm{C}-$

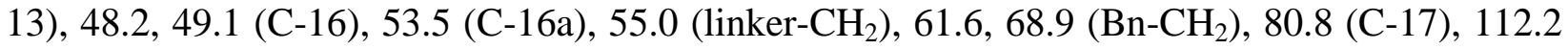
(C-2), 114.4 (C-4), 122.6 (triazol-CH), 126.6 (C-1), 127.4 (C-2' and -6'), 127.6 (C-4'), 128.3 (C3' and -5'), 132.4 (C-10), 137.3 (C-5 and C-1'), 147.6 (triazol-C), 156.0 (C-3). 


\subsubsection{3-Benzyloxy-16a-(4'-cyclopropyl-1 'H-1',2',3'-triazol-1'-yl)methylestra-1,3,5(10)-trien-}

$17 a-o l(28 a)$

Compound 20 (420.0 mg, $1 \mathrm{mmol}$ ) and cyclopropylacetylene ( $2 \mathrm{mmol}, 0.22 \mathrm{ml})$ were used for the synthesis as described in Section 2.3. The crude product was chromatographed on silica gel with ethyl acetate $/ \mathrm{CH}_{2} \mathrm{Cl}_{2}(1: 99 \mathrm{v} / \mathrm{v})$ to yield pure 28a (305 mg, 63\%) as white crystals. Mp: 143-144 ${ }^{\circ} \mathrm{C} ; R_{\mathrm{f}}=0.40$ (ss B). (Found $\mathrm{C}, 77.15 ; \mathrm{H}, 7.53 . \mathrm{C}_{31} \mathrm{H}_{37} \mathrm{~N}_{3} \mathrm{O}_{2}$ (483.64) requires $\mathrm{C}, 76.98 ; \mathrm{H}$, 7.71\%). ${ }^{1} \mathrm{H} \mathrm{NMR}\left(\delta, \mathrm{ppm}, \mathrm{CDCl}_{3}\right): 0.74$ (s, 3H, 18- $\left.\mathrm{H}_{3}\right), 0.87$ and $0.97(2$ x s, 2 x $2 \mathrm{H}, 2$ "- and 3"$\left.\mathrm{H}_{2}\right), 2.85\left(\mathrm{~m}, 2 \mathrm{H}, 6-\mathrm{H}_{2}\right), 3.63(\mathrm{~d}, 1 \mathrm{H}, J=5.0 \mathrm{~Hz}, 17-\mathrm{H}), 4.26(\mathrm{dd}, 1 \mathrm{H}, J=13.5 \mathrm{~Hz}, J=5.5 \mathrm{~Hz}$, $\left.16 \mathrm{a}-\mathrm{H}_{2}\right), 4.60\left(\mathrm{t}, 1 \mathrm{H}, J=13.5 \mathrm{~Hz}, 16 \mathrm{a}-\mathrm{H}_{2}\right), 5.03\left(\mathrm{~s}, 2 \mathrm{H}, \mathrm{Bn}-\mathrm{H}_{2}\right), 6.72(\mathrm{~d}, 1 \mathrm{H}, J=2.0 \mathrm{~Hz}, 4-\mathrm{H})$, $6.78(\mathrm{dd}, 1 \mathrm{H}, J=8.5 \mathrm{~Hz}, J=2.5 \mathrm{~Hz}, 2-\mathrm{H}), 7.22(\mathrm{~d}, 1 \mathrm{H}, J=8.5 \mathrm{~Hz}, 1-\mathrm{H}), 7.32(\mathrm{t}, 1 \mathrm{H}, J=7.5 \mathrm{~Hz}$, $\left.4^{\prime}-\mathrm{H}\right), 7.38$ (t, 3H, $J=7.5 \mathrm{~Hz}, 3^{\prime}$ - and 5'- $\mathrm{H}$, triazol-H), 7.43 (d, $2 \mathrm{H}, J=7.5 \mathrm{~Hz}, 2^{\prime}-$ and $\left.6{ }^{\prime}-\mathrm{H}\right) .{ }^{13} \mathrm{C}$ NMR ( $\delta, p p m, \mathrm{CDCl}_{3}$ ): 6.5 (C-1"), 7.9 (2C, C-2" and -3"), 17.1 (C-18), 26.0, 27.9, 28.9, 29.8, 31.2, 38.9, 42.3, 43.5, 46.3 (C-16a), 47.0 (C-16), 50.7 (C-13), 69.9 (Bn- $\left.\mathrm{CH}_{2}\right), 78.7$ (C-17), 112.2 (C-2), 114.8 (C-4), 120.8 (triazol-CH)), 126.3 (C-1), 127.4 (C-2' and -6'), 127.4 (C-4'), 128.5 (C-3' and -5'), 132.5 (C-10), 137.2 (C-1'), 137.9 (C-5), 149.6 (triazol-C), 156.7 (C-3).

2.3.44. 3-Benzyloxy-16a-(4'-cyclopentyl-1'H-1',2',3'-triazol-1'-yl)methylestra-1,3,5(10)-trien$17 a-o l(28 b)$

Compound 20 ( $420.0 \mathrm{mg}, 1 \mathrm{mmol})$ and cyclopentylacetylene $(2 \mathrm{mmol}, 0.22 \mathrm{ml})$ were used for the synthesis as described in Section 2.3. The crude product was chromatographed on silica gel with ethyl acetate $/ \mathrm{CH}_{2} \mathrm{Cl}_{2}(2.5: 97.5 \mathrm{v} / \mathrm{v})$ to yield pure $\mathbf{2 8 b}(417 \mathrm{mg}, 82 \%)$ as white crystals. Mp: $197-199{ }^{\circ} \mathrm{C} ; R_{\mathrm{f}}=0.42$ (ss B). (Found: $\mathrm{C}, 77.62 ; \mathrm{H}, 7.85 . \mathrm{C}_{33} \mathrm{H}_{41} \mathrm{~N}_{3} \mathrm{O}_{2}(511.70$ ) requires $\mathrm{C}, 77.46$; $\mathrm{H}, 8.08 \%) .{ }^{1} \mathrm{H}$ NMR $\left(\delta, \mathrm{ppm}, \mathrm{CDCl}_{3}\right): 0.76$ (s, 3H, 18- $\left.\mathrm{H}_{3}\right), 2.85$ (m, 2H, 6- $\left.\mathrm{H}_{2}\right), 3.20$ (s, 1H, 1"H), $3.66(\mathrm{~d}, 1 \mathrm{H}, J=5.0 \mathrm{~Hz}, 17-\mathrm{H}), 4.29\left(\mathrm{dd}, 1 \mathrm{H}, J=13.5 \mathrm{~Hz}, J=5.5 \mathrm{~Hz}, 16 \mathrm{a}-\mathrm{H}_{2}\right), 4.62(\mathrm{dd}, 1 \mathrm{H}$, $\left.J=13.5 \mathrm{~Hz}, J=9.5 \mathrm{~Hz}, 16 \mathrm{a}-\mathrm{H}_{2}\right), 5.04\left(\mathrm{~s}, 2 \mathrm{H}, \mathrm{Bn}-\mathrm{H}_{2}\right), 6.72(\mathrm{~s}, 1 \mathrm{H}, 4-\mathrm{H}), 6.78(\mathrm{dd}, 1 \mathrm{H}, J=8.5 \mathrm{~Hz}$, $J=2.5 \mathrm{~Hz}, 2-\mathrm{H}), 7.21(\mathrm{~d}, 1 \mathrm{H}, J=8.5 \mathrm{~Hz}, 1-\mathrm{H}), 7.31\left(\mathrm{t}, 1 \mathrm{H}, J=7.0 \mathrm{~Hz}, 4^{\prime}-\mathrm{H}\right), 7.37$ (t, 2H, $J=7.0$ $\mathrm{Hz}, 3^{\prime}$ '- and 5'-H), 7.43 (d, 2H, $J=7.0 \mathrm{~Hz}, 2$ '- and 6'-H). ${ }^{13} \mathrm{C}$ NMR ( $\left.\delta, \mathrm{ppm}, \mathrm{CDCl}_{3}\right): 17.3(\mathrm{C}-$ 18), 25.2 (2C), 26.1, 28.0, 29.1, 29.8 (2C), 31.3, 33.2, 36.8 (C-1"), 39.0, 42.4, 43.6, 46.4 (C-16a), 47.2 (C-16), 50.6 (C-13), 70.1 (Bn-CH $), 79.0$ (C-17), 112.4 (C-2), 115.0 (C-4), 126.3 (C-1), 127.4 (C-2' and -6'), 127.8 (C-4'), 128.5 (C-3' and -5'), 133.0 (C-10), 137.5 (C-1'), 137.9 (C-5), $156.9(\mathrm{C}-3)$. 
2.3.45. 3-Benzyloxy-16a-(4-cyclohexyl-1H-1,2,3-triazol-1-yl)methyl-estra-1,3,5(10)-trien-17a-ol (28c)

Compound 20 (420.0 mg, $1 \mathrm{mmol})$ and cyclohexylacetylene $(2 \mathrm{mmol}, 0.22 \mathrm{ml})$ were used for the synthesis as described in Section 2.3. The crude product was chromatographed on silica gel with ethyl acetate $/ \mathrm{CH}_{2} \mathrm{Cl}_{2}(2.5: 97.5 \mathrm{v} / \mathrm{v})$ to yield pure $28 \mathrm{c}$ (200 $\left.\mathrm{mg}, 76 \%\right)$ as a white solid. $\mathrm{Mp}$ : 223-225 ${ }^{\circ} \mathrm{C} ; R_{\mathrm{f}}=0.44$ (ss B). (Found C, 77.82; H, 8.35. $\mathrm{C}_{34} \mathrm{H}_{43} \mathrm{~N}_{3} \mathrm{O}_{2}$ (525.72) requires C, 77.68; $\mathrm{H}, 8.24 \%) .{ }^{1} \mathrm{H}$ NMR $\left(\delta, \mathrm{ppm}, \mathrm{CDCl}_{3}\right): 0.75$ (s, 3H, 18- $\left.\mathrm{H}_{3}\right), 2.84(\mathrm{~m}, 3 \mathrm{H}, 6-\mathrm{H} 2,1$ '- $\mathrm{H}), 3.64(\mathrm{~s}, 1 \mathrm{H}$, $17-\mathrm{H}), 4.37\left(\mathrm{~m}, 1 \mathrm{H}, 16 \mathrm{a}-\mathrm{H}_{2}\right), 4.69\left(\mathrm{~m}, 1 \mathrm{H}, 16 \mathrm{a}-\mathrm{H}_{2}\right), 5.03\left(\mathrm{~s}, 2 \mathrm{H}, \mathrm{Bn}-\mathrm{H}_{2}\right), 6.72(\mathrm{~d}, 1 \mathrm{H}, J=1.5 \mathrm{~Hz}$, 4-H), $6.78(\mathrm{dd}, 1 \mathrm{H}, J=8.5 \mathrm{~Hz}, J=2.5 \mathrm{~Hz}, 2-\mathrm{H}), 7.22(\mathrm{~d}, 1 \mathrm{H}, J=8.5 \mathrm{~Hz}, 1-\mathrm{H}), 7.32$ (t, $1 \mathrm{H}, J=$ $\left.7.0 \mathrm{~Hz}, 4^{\prime}-\mathrm{H}\right), 7.38$ (t, 2H, $J=7.0 \mathrm{~Hz}, 3^{\prime}-$ and 5'-H), 7.43 (d, 2H, $J=7.0 \mathrm{~Hz}, 2^{\prime}-$ and 6'-H

2.3.46. 3-Benzyloxy-16a-(4-phenyl-1H-1,2,3-triazol-1-yl)methyl-estra-1,3,5(10)-trien-17a-ol (28d)

Compound $20(420.0 \mathrm{mg}, 1 \mathrm{mmol})$ and phenylacetylene $(2 \mathrm{mmol}, 0.22 \mathrm{ml})$ were used for the synthesis as described in Section 2.3. The crude product was chromatographed on silica gel with ethyl acetate $/ \mathrm{CH}_{2} \mathrm{Cl}_{2}(5: 95 \mathrm{v} / \mathrm{v})$ to yield pure $\mathbf{2 8 d}$ (337 mg, 64\%) as a white solid. Mp: 205-206 ${ }^{\circ} \mathrm{C} ; R_{\mathrm{f}}=0.46$ (ss B). (Found C, 78.42; H, 7.32. $\mathrm{C}_{34} \mathrm{H}_{37} \mathrm{~N}_{3} \mathrm{O}_{2}(519.68)$ requires $\mathrm{C}, 78.58 ; \mathrm{H}, 7.18 \%$ ). ${ }^{1} \mathrm{H}$ NMR ( $\delta$, ppm, $\left.\mathrm{CDCl}_{3}\right): 0.76\left(\mathrm{~s}, 3 \mathrm{H}, 18-\mathrm{H}_{3}\right), 2.87\left(\mathrm{~m}, 2 \mathrm{H}, 6-\mathrm{H}_{2}\right), 3.68(\mathrm{~d}, 1 \mathrm{H}, J=5.0 \mathrm{~Hz}, 17-$ H), $4.41\left(\mathrm{dd}, 1 \mathrm{H}, J=13.5 \mathrm{~Hz}, J=5.5 \mathrm{~Hz}, 16 \mathrm{a}-\mathrm{H}_{2}\right), 4.69\left(\mathrm{t}, 1 \mathrm{H}, J=13.5 \mathrm{~Hz}, 16 \mathrm{a}-\mathrm{H}_{2}\right), 5.04(\mathrm{~s}, 2 \mathrm{H}$, Bn-H $\left.\mathrm{H}_{2}\right), 6.73$ (s, 1H, 4-H), 6.79 (dd, 1H, $\left.J=8.0 \mathrm{~Hz}, J=2.0 \mathrm{~Hz}, 2-\mathrm{H}\right), 7.22(\mathrm{~d}, 1 \mathrm{H}, J=8.0 \mathrm{~Hz}, 1-$ H), 7.38 (m, 8H, 2'-, 3'-, 4'-, 5'- and 6'-H, 3'-, 4'-- and 5'-H), 7.84 (d, 2H, J = 7.5 Hz, 2"'- and 6"-H), 7.89 (s, $1 \mathrm{H}$, triazol-H). ${ }^{13} \mathrm{C}$ NMR ( $\delta$, ppm, $\mathrm{CDCl}_{3}$ ): 17.1 (C-18), 26.0, 27.9, 29.8, 31.2,

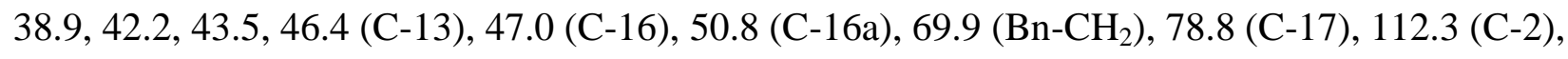
114.8 (C-4), 120.7 (triazol-CH), 125.7 (C-2' and -6'), 126.3 (C-1), 127.4 (C-2" and -6"), 127.8 (C-4'), 128.3 (C-4”), 128.5 (C-3” and -5”), 128.9 (C-3' and -5'), 130.2 (C-10), 132.8 (C-1'), 137.3 (C-1”), 137.9 (C-5), 147.1 (triazol-C), 156.7 (C-3).

2.3.47. 3-Benzyloxy-16a-[4'-(4',-nitro-benzoyloxymethyl)-1'H-1',2',3'-triazol-1'-yl]methylestra1,3,5(10)-trien-17a-ol (28e) 
Compound 20 (420 mg, $1 \mathrm{mmol}$ ) and propargyl 4-nitrobenzoate ( $2 \mathrm{mmol}, 210 \mathrm{mg}$ ) were used for the synthesis as described in Section 2.3. The crude product was chromatographed on silica gel with ethyl acetate/ $\mathrm{CH}_{2} \mathrm{Cl}_{2}(5: 95 \mathrm{v} / \mathrm{v})$ to yield pure $28 \mathrm{e}(610 \mathrm{mg}, 98 \%)$ as a yellow solid. Mp: $75-77{ }^{\circ} \mathrm{C} ; R_{\mathrm{f}}=0.45$ (ss B). (Found C, 69.57; H, 61.32. $\mathrm{C}_{36} \mathrm{H}_{38} \mathrm{~N}_{4} \mathrm{O}_{6}(622.71)$ requires $\mathrm{C}, 69.44 ; \mathrm{H}$, 6.15\%). ${ }^{1} \mathrm{H}$ NMR ( $\delta$, ppm, DMSO-d $\left.\mathrm{d}_{6}\right): 0.66$ (s, 3H, 18- $\left.\mathrm{H}_{3}\right), 2.71\left(\mathrm{~m}, 2 \mathrm{H}, 6-\mathrm{H}_{2}\right), 3.57$ (s, 1H, 16H), $4.29\left(\mathrm{dd}, 1 \mathrm{H}, J=13.5 \mathrm{~Hz}, J=8.5 \mathrm{~Hz}, 16 \mathrm{a}-\mathrm{H}_{2}\right), 4.47(\mathrm{dd}, 1 \mathrm{H}, J=13.5 \mathrm{~Hz}, J=8.5 \mathrm{~Hz}, 16 \mathrm{a}-$ $\left.\mathrm{H}_{2}\right), 4.85(\mathrm{~d}, 1 \mathrm{H}, J=5.0 \mathrm{~Hz}, 17-\mathrm{H}), 5.44\left(\mathrm{~s}, 2 \mathrm{H}, \mathrm{Bn}-\mathrm{H}_{2}\right), 6.65$ (s, $\left.1 \mathrm{H}, 4-\mathrm{H}\right), 6.72(\mathrm{~d}, 1 \mathrm{H}, J=8.5$ Hz, 2-H), 7.14 (d, 1H, J = 8.5 Hz, 1-H), 7.29 (t, 1H, $\left.J=7.5 \mathrm{~Hz}, 4^{\prime}-\mathrm{H}\right), 7.35$ (t, 2H, $J=7.5 \mathrm{~Hz}, 3^{\prime}-$ and 5'-H), 7.40 (d, 2H, $J=7.5 \mathrm{~Hz}, 2^{\prime}$ '- and 6'-H), 8.17 (d, 2H, $J=8.5 \mathrm{~Hz}, 3$ '- and 5'-H), 8.28 (s, $1 \mathrm{H}$, triazol $\mathrm{H}), 8.31(\mathrm{~d}, 2 \mathrm{H}, J=8.5 \mathrm{~Hz}, 2$ "- and $6 "-\mathrm{H}) .{ }^{13} \mathrm{C}$ NMR $(\delta, \mathrm{ppm}$, DMSO-d 6 ): $16.9(\mathrm{C}-$ 18), 25.6, 27.5, 28.4, 29.2, 31.1, 38.5, 39.8, 39.9, 43.2, 45.9 (C-16a), 46.2 (C-16), 53.4 (C-13), 58.7 (linker $\mathrm{CH}_{2}$ ), 68.9 (Bn- $\mathrm{CH}_{2}$ ), 78.0 (C-17), 112.1 (C-2), 114.4 (C-4), 123.8 (C-2" and -6"), 125.0 (triazol CH), 126.1 (C-1), 127.4 (C-2' and -6'), 127.5 (C-4'), 128.3 (C-3' and -5'), 130.6 (C-3" and -5”), 132.3 (C-10), 134.7 (C-1'), 137.3 (C-5), 141.0 (C-1"), 150.2 (triazol C), 156.0 (C-3), $163.9(\mathrm{C}=\mathrm{O})$.

2.3.48. 3-Benzyloxy-16a-(4'-hydroxymethyl-1 'H-1',2',3'-triazol-1'-yl)methylestra-1,3,5(10)trien-17 $\alpha$-ol (28f)

Compound 28e (220 mg, $0.5 \mathrm{mmol})$ was dissolved in methanol $(10 \mathrm{ml})$ containing $\mathrm{NaOCH}_{3}(14$ $\mathrm{mg}, 0.25 \mathrm{mmol}$ ), and the solution was allowed to stand for $24 \mathrm{~h}$. It was then diluted with water, and the precipitate separating out was filtered off and recrystallized from methanol to afford $\mathbf{2 8 f}$ (126 mg, 53\%) as a white crystalline product. Mp: 86-88 ${ }^{\circ} \mathrm{C} ; R_{\mathrm{f}}=0.25$ (ss B). (Found C, 73.68; $\mathrm{H}, 7.63 . \mathrm{C}_{29} \mathrm{H}_{35} \mathrm{~N}_{3} \mathrm{O}_{3}$ (473.61) requires $\left.\mathrm{C}, 73.54 ; \mathrm{H}, 7.45 \%\right) .{ }^{1} \mathrm{H}$ NMR ( $\delta$, ppm, DMSO-d $\left.\mathrm{d}_{6}\right): 0.68$ (s, 3H, 18- $\left.\mathrm{H}_{3}\right), 2.74\left(\mathrm{~m}, 2 \mathrm{H}, 6-\mathrm{H}_{2}\right), 3.58(\mathrm{brs}, 1 \mathrm{H}, \mathrm{OH}), 4.26\left(\mathrm{t}, 1 \mathrm{H}, J=8.5 \mathrm{~Hz}, 16 \mathrm{a}-\mathrm{H}_{2}\right), 4.43$ (dd, $\left.1 \mathrm{H}, J=13.0 \mathrm{~Hz}, J=7.0 \mathrm{~Hz}, 16 \mathrm{a}-\mathrm{H}_{2}\right), 4.51\left(\mathrm{~d}, 2 \mathrm{H}, J=5.0 \mathrm{~Hz}\right.$, linker $\left.\mathrm{H}_{2}\right), 4.85(\mathrm{~d}, 1 \mathrm{H}, J=4.0 \mathrm{~Hz}$, 17-H), 5.04 (s, 2H, Bn- $\mathrm{H}_{2}$ ), 5.13 (brs, 1H, OH), 6.68 (s, 1H, 4-H), 6.74 (d, 1H, J = 8.5 Hz, 2-H), 7.17 (d, 1H, $J=8.5 \mathrm{~Hz}, 1-\mathrm{H}), 7.31$ (d, 1H, $\left.J=7.0 \mathrm{~Hz}, 4^{\prime}-\mathrm{H}\right), 7.37$ (t, $2 \mathrm{H}, J=7.0 \mathrm{~Hz}, 3^{\prime}$ '- and 5'H), $7.42\left(\mathrm{~d}, 2 \mathrm{H}, J=7.0 \mathrm{~Hz}, 2^{\prime}-\right.$ and $\left.6^{\prime}-\mathrm{H}\right), 7.97$ (s, $1 \mathrm{H}$, triazol H). ${ }^{13} \mathrm{C}$ NMR $\left(\delta\right.$, ppm, DMSO-d $\left.\mathrm{d}_{6}\right)$ : 16.9 (C-18), 25.6, 27.5, 28.5, 29.2, 31.1, 38.5, 40.7, 43.2, 45.9, 46.2 (C-16), 47.9 (C-13), 50.6 (C16a), 55.0 (linker $\mathrm{CH}_{2}$ ), $68.9\left(\mathrm{Bn}^{-\mathrm{CH}_{2}}\right), 78.0$ (C-17), 112.1 (C-2), $114.4(\mathrm{C}-4), 122.7$ (triazol 
CH), 126.1 (C-1), 127.4 (C-2' and -6'), 127.6 (C-4'), 128.3 (C-3' and -5'), 132.4 (C-10), 137.3 (C-1'), 137.4 (C-5), 147.6 (triazol C), 156.0 (C-3).

\subsection{Determination of the antiproliferative activities}

The growth-inhibitory effects of the compounds were tested in vitro by means of the MTT assay against a gynecological panel containing two breast cancer cell lines (MCF-7, MD-MB231) and two cell lines isolated from cervical malignancies (HeLa, SiHa) [11]. All cell lines were obtained from the European Collection of Cell Cultures (Salisbury, UK). The cells were maintained in minimal essential medium supplemented with $10 \%$ fetal bovine serum (FBS), $1 \%$ non-essential amino acids and an antibiotic-antimycotic mixture (AAM). All chemicals, if otherwise not specified, were purchased from Sigma-Aldrich Ltd. (Budapest, Hungary). All cell lines were grown in a humidified atmosphere of $5 \% \mathrm{CO}_{2}$ at $37{ }^{\circ} \mathrm{C}$. For pharmacological investigations, $10 \mathrm{mM}$ stock solutions of the tested compounds were prepared with dimethyl sulfoxide (DMSO). The highest applied DMSO concentration of the medium (0.3\%) did not have any substantial effect on the determined cellular functions. Cells were seeded into 96-well plates (5000 cells/well), allowed to stand overnight under cell culturing conditions, and the medium containing the tested compounds at two final concentrations $(10$ or $30 \mu \mathrm{M})$ was then added. After a 72-hour incubation viability was determined by the addition of $20 \mu 1$ 3-(4,5-dimethylthiazol-2yl)-2,5-diphenyltetrazolium bromide (MTT) solution $(5 \mathrm{mg} / \mathrm{ml}$ ). The formazan crystals precipitated in $4 \mathrm{~h}$ were solubilized in DMSO and the absorbance was determined at $545 \mathrm{~nm}$ with an ELISA plate reader utilizing untreated cells as controls. The most effective compounds eliciting at least $60 \%$ growth inhibition at $10 \mu \mathrm{M}$ were tested again with a set of dilutions $(0.3-30$ $\mu \mathrm{M}$ ) in order to determine the $\mathrm{IC}_{50}$ values by means of Graphpad Prism 4.0 (Graphpad Software; San Diego, CA, US). These promising compounds were additionally tested using nonmalignant murine fibroblasts (NIH-3T3) to obtain preliminary data concerning cancer selectivity of the tested molecules. Two independent experiments were performed with 5 parallel wells and cisplatin (Ebewe $\mathrm{GmbH}$, Unterach, Austria), an agent administered clinically in the treatment of certain gynecological malignancies, was used as reference compound.

\section{Results and discussion}

3.1. Synthetic studies 


\subsection{Determination of the antiproliferative properties of the 16-triazolylmethyl diastereomers}

We have published recently that introduction of a substituted triazole moiety onto different positions of the estrane skeleton might increase the antiproliferative properties of estrone derivatives [12]. It was also established that the presence of certain alkyl or aralkyl protecting groups at the phenolic $\mathrm{OH}$ function is advantageous. Concerning that 16hydroxymethylene-17-hydroxy derivatives of estrone-3-methyl ether or 3-benzyl ether (5a-12a) displayed substantial cytostatic potential against different types of breast cancer cell lines, these compounds might be suitable for directed modifications with the aim of developing potentially more active antiproliferative steroidal derivatives [13]. In the light of the above-mentioned recent observations, here we aimed to combine the substituted triazole and the 16,17-disubstituted estrone 3-ether moieties. The present study included an evaluation of the direct antiproliferative capacities of the newly synthesized heterocyclic compounds $(\mathbf{2 1 a}-\mathbf{f}, \mathbf{2 2} \mathbf{a}-\mathbf{f}, 23 \mathbf{a}-\mathbf{f}, \mathbf{2 4 a}-\mathbf{f}$ and 25a-f, 26a-f, 27a-f, 28a-f). The antiproliferative activities were determined in vitro by means of MTT assays against human adherent cervical (SiHa, HeLa) and breast cancer (MCF-7 and MDA-MB-231) cell lines. 
The antiproliferative activities of the newly synthesized heterocyclic compounds depended on the nature of the protecting group at the 3-hydroxy function and on the orientation of the substituents at C-16 and C-17. In general, the 3-methyl ethers (21-24) exhibited weak antiproliferative action; none of them exerted any substantial effect at $10 \mu \mathrm{M}$ (Table 1). All diastereomers of the 3-benzyl ether series (25-28) proved to be more potent in comparison with their 3-methyl ether counterparts (Table 2). This is in agreement with our earlier results [14]. Based on the substantial difference of the two groups, i.e. that of 3-methyl ethers and 3-benzyl ethers, it can be concluded that only the latter derivatives are promising from pharmacological point of view.

Concerning the orientation of the substituents at position C-16 and C-17, the $16 \beta, 17 \beta-$ derivatives (25a-f) displayed outstanding growth-inhibitory properties. Two derivatives bearing similar cycloalkyl groups at position C-4' displayed substantial selective antiproliferative action against the triple-negative breast cancer cell line MDA-MB-231 with $\mathrm{IC}_{50}$ values in the low micromolar range. It should be underlined that $25 \mathbf{b}$ and $25 \mathbf{c}$ did not significantly influence the proliferation of other cell lines tested, including the non-cancerous fibroblast. Although both the 4'-cyclohexyl (25c) and the 4'-phenyl derivative (25d) have six-membered substituents, their cytostatic behavior is completely different. This might be attributed to the different steric structure of the two rings (chair or planar) at C-4'. Compound 25d exerted potent

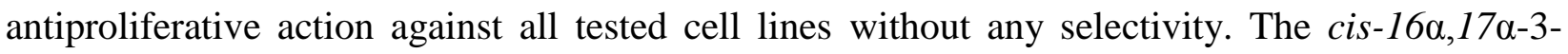
benzyl ethers (28a-f) were less potent than their $\beta, \beta$-counterparts (25a-f), except for $\mathbf{2 8 d}$, which behaved similarly to its diastereomer 25d. The trans-16 $\beta, 17 \alpha$-isomers (27a-f) exhibited activity exclusively on the breast cancer cell lines. Surprisingly, the tendency observed earlier (in the case of compounds 25a-f) concerning the nature of C-4' substituent was not valid here. Only 26a and 26e inhibited cell growth markedly, but with no tumor selectivity. It's worth mentioning that trans-16 $\alpha, 17 \beta$ isomer $26 \mathbf{c}$ was the sole compound, which inhibited the proliferation of HPV 16+ squamous cell carcinoma $\mathrm{SiHa}$, showing an $\mathrm{IC}_{50}$ value comparable with that of cisplatin.

In view of the cell lines, it should be noted that triple-negative breast cancer cell line MDA-MB231 proved to be the most sensitive and all calculated $\mathrm{IC}_{50}$ values were lower than that of the reference agent cisplatin $(19.1 \mu \mathrm{M})$.

Regarding the present and earlier results obtained for 16,17-disubstituted 3-benzyl ethers, it can be stated that introduction of a substituted triazolyl moiety onto the C-16 methylene group of the 
cis isomers proved to be advantageous. In the case of compounds $\mathbf{2 5 b}$ and $\mathbf{2 5 c}$, both the antiproliferative potential and the tumor selectivity were markedly improved.

\section{Acknowledgement}

The work of Anita Kiss was supported by a PhD Fellowship of the Talentum Fund of Richter Gedeon Plc. (Budapest). Financial support from the Economic Development and Innovation Operative Programme of Hungary (GINOP-2.3.2-15-2016-00038) and Ultrafast physical processes in atoms, molecules, nanostructures and biological systems (No: EFOP-3.6.2.2017-00005) is gratefully acknowledged. This research was supported by the Hungarian Scientific Research Fund (OTKA K113150). Ministry of Human Capacities, Hungary grant 20391-3/2018/FEKUSTRAT is acknowledged. 


\section{References}

[1] Tietze LF, Bell HP, Chandrasekhar S, Natural product hybrids as new leads drug discovery. Angew Chem Int Ed 2003;42:3996-4028.

[2] Mehta G, Singh V, Hybrid system through natural product leads: An approach towards new molecular entities. Chem Soc Rev 2002;31:324-334.

[3] Adamec J, Beckert R, Weiß D, Klimešová V, Waisser K, Möllmann U, Kaustová J, Buchta V. Hybride molecules of estrone: New Compound with potential antibacterial, antifungal and antiproliferative activities. Bioorg Med Chem 2007;15:2898-2906.

[4] Kuduk, SD, Zheng FF, Sepp-Lorenzino L, Rosen N, Danishefsy SJ. Synthesis and Evaluation of geldanamycin-estradiol hybrids. Bioorg Med Chem 1999;9:1233-1238.

[5] Gupta A, Saha P, Descôteaux C, Leblanc V, Asselin É, Bérubé G. Design, synthesis and biological evaluation of estradiol-chlorambucil hybrids as anticancer agents. Bioorg Med Chem Lett 2010;20:1614-1618.

[6] Schneider Gy, Vass A, Vincze I, Sohár P. Neighbouring Group Partcipation in the 16Hydroxymethyl-3-methoxyestre-1,3,5(10)-trien-17ß-ol Series. Liebigs Ann Chem 1988;267-273.

[7] Schneider Gy, Hackler L, Sohár P. Preparation of 16 $\alpha$-Hydroxymethyl-3-methoxyestra1,3,5(10)-trien 17 $\alpha$-ol and Solvolysis Investigation. Liebigs Ann Chem 1988;679-683.

[8] Tapolcsányi P, Wölfling J, Falkay G, Márki Á, Minorics R, Schneider Gy. Synthesis and receptor-binding examination of 16-hydroxymethyl-3,17-estradiol stereoisomers. Steroids 2002;67:671-678.

[9] Meldal M, Tornøe CW. Cu-Catalyzed azide-alkyne cycloaddition. Chem Rev.

2008;108:2952-3015.

[10] Szájli Á, Wölfling J, Mernyák E, Minorics R, Márki Á, Falkay G, Schneider Gy. Neighbouring group participation Part 16. Stereoselective synthesisi and receptor-binding examination of the four stereoisomers of 16-bromomethyl-3,17-estradiols. Steroids 2006; $71: 141-153$.

[11] Mosmann T. Rapid colorimetrc assay for cellural growth and survival: application to prolyferation and cytotoxicity assay. J immunol Methods 1983;65:55-63. 
[12] Mernyák E, Kovács I, Minorics R, Sere P, Czégány Sinka I, Wölfling, Schneider G, Újfaludi Zs, Boros I, Ocsovszki I, Varjj ga M, Zupkó I. Synthesis of trans-16-triazolyl-13amethyl-17-estradiol diastereomers and the effects of structural modifications on their in vitro antiproliferative activities. J Steroid Biochem Mol Biol 2015;150:123-134.

[13] Sinka I, Kiss A, Mernyák E, Wölfling E, Schneider G. Ocsovszki I, Kuo CY, Wang HC, Zupkó I. Antiproliferative and antimetastatic properties of 3-benzyloxy-16-hydroxymethyleneestardiol analogs agains breast cancer cell lines. Eur J Pharm Sciences 2018;123:362-370. [14] Frank E, Molnar J, Zupko I, Kadar Z, Wölfling J. Synthesis of novel steroidal 17 $\alpha$-triazolyl derivatives via $\mathrm{Cu}(\mathrm{I})$-catalyzed azide-alkyne cycloaddition, and an evaluation of their cytotoxic activity in vitro. Steroids 2011;76:1141-1148. 


\section{Legends for Schemes and Tables}

Scheme 1 Reagents and conditions: (i) NaOMe, HCOOEt, anhydrous toluene, $50{ }^{\circ} \mathrm{C}$; (ii) $\mathrm{KBH}_{4}$, $\mathrm{MeOH}$; (iii) $\mathrm{KOAc}, \mathrm{CH}_{3} \mathrm{COOH}, \mathrm{NaOMe} / \mathrm{MeOH}$.

Scheme 2 Reagents and conditions: (i) appropriate alkyne, TEA, $\mathrm{CuI}, \mathrm{CH}_{2} \mathrm{Cl}_{2}, 40{ }^{\circ} \mathrm{C}, 24 \mathrm{~h}$; (ii) $\mathrm{NaOMe}, \mathrm{MeOH}, 24 \mathrm{~h}$.

Table 1 Antiproliferative activities of compounds $21 \mathbf{a}-\mathbf{f}, \mathbf{2 2} \mathbf{a}-\mathbf{f}, \mathbf{2 3 a}-\mathbf{f}$ and $24 \mathbf{a}-\mathbf{f}$

Table 2 Antiproliferative activities of compounds $25 \mathbf{a}-\mathbf{f}, 26 \mathbf{a}-\mathbf{f}, 27 \mathbf{a}-\mathbf{f}$ and $28 \mathbf{a}-\mathbf{f}$ 
Table 1

\begin{tabular}{|c|c|c|c|c|c|}
\hline & & $\begin{array}{l}\text { Grov } \\
{[\mathrm{c}}\end{array}$ & $\begin{array}{l}\text { Inhibition, } \% \\
\text { ulated } \mathrm{IC}_{50}(\mu\end{array}$ & & \\
\hline & $\begin{array}{r}\text { Conc. } \\
(\mu \mathrm{M})\end{array}$ & HeLa & $\mathrm{SiHa}$ & MCF-7 & $\begin{array}{c}\text { MDA-MB- } \\
231\end{array}$ \\
\hline 21 & & & & & \\
\hline & 10 & $<20$ & $21.28 \pm 1.88$ & $<20$ & $<20$ \\
\hline $\mathbf{a}$ & 30 & $<20$ & $28.71 \pm 2.20$ & $46.42 \pm 1.47$ & $<20$ \\
\hline & 10 & $<20$ & $<20$ & $<20$ & $<20$ \\
\hline b & 30 & $39.86 \pm .38$ & $<20$ & $57.42 \pm 1.77$ & $29.88 \pm 1.57$ \\
\hline & 10 & $<20$ & $<20$ & $<20$ & $<20$ \\
\hline c & 30 & $40.22 \pm 1.02$ & $<20$ & $70.84 \pm 1.55$ & $37.96 \pm 1.55$ \\
\hline & 10 & $<20$ & $<20$ & $<20$ & $<20$ \\
\hline d & 30 & $44.16 \pm 0.48$ & $<20$ & $54.93 \pm 1.78$ & $38.28 \pm 1.84$ \\
\hline & 10 & $<20$ & $23.91 \pm 1.61$ & $34.23 \pm 3.10$ & $<20$ \\
\hline $\mathrm{e}$ & 30 & $37.18 \pm 1.65$ & $54.72 \pm 0.48$ & $76.26 \pm 0.72$ & $35.93 \pm 2.13$ \\
\hline$f$ & 10 & $<20$ & $28.06 \pm 1.99$ & $29.45 \pm 1.67$ & $<20$ \\
\hline 1 & 30 & $41.03 \pm 0.77$ & $57.69 \pm 1.12$ & $70.23 \pm 1.35$ & $34.81 \pm 2.88$ \\
\hline 22 & & & & & \\
\hline 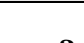 & 10 & $<20$ & $25.55 \pm 1.01$ & $<20$ & $<20$ \\
\hline a & 30 & $<20$ & $34.78 \pm 2.47$ & $57.43 \pm 1.91$ & $<20$ \\
\hline b & 10 & $<20$ & $<20$ & $<20$ & $<20$ \\
\hline 10 & 30 & $<20$ & $26.57 \pm 2.26$ & $67.59 \pm 1.65$ & $<20$ \\
\hline 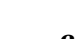 & 10 & $<20$ & $<20$ & $<20$ & $<20$ \\
\hline $\mathbf{c}$ & 30 & $<20$ & $29.90 \pm 2.59$ & $69.68 \pm 0.77$ & $<20$ \\
\hline d & 10 & $<20$ & $<20$ & $<20$ & $<20$ \\
\hline a & 30 & $<20$ & $29.96 \pm 1.79$ & $70.75 \pm 1.05$ & $14.54 \pm 1.32$ \\
\hline & 10 & $<20$ & $<20$ & $<20$ & $<20$ \\
\hline e & 30 & $<20$ & $38.69 \pm 2.09$ & $63.12 \pm 2.14$ & $<20$ \\
\hline$f$ & 10 & $<20$ & $<20$ & $22.02 \pm 1.61$ & $<20$ \\
\hline $\mathbf{I}$ & 30 & $<20$ & $37.79 \pm 1.04$ & $50.94 \pm 1.55$ & $<20$ \\
\hline 23 & & & & & \\
\hline & 10 & $<20$ & $<20$ & $<20$ & $<20$ \\
\hline $\mathbf{a}$ & 30 & $31.14 \pm 1.28$ & $<20$ & $28.72 \pm 0.93$ & $25.08 \pm 3.15$ \\
\hline h & 10 & $<20$ & $<20$ & $<20$ & $<20$ \\
\hline b & 30 & $58.25 \pm 2.03$ & $<20$ & $48.01 \pm 1.31$ & $<20$ \\
\hline & 10 & $<20$ & $30.97 \pm 2.69$ & $<20$ & $<20$ \\
\hline c & 30 & $<20$ & $33.89 \pm 2.35$ & $<20$ & $<20$ \\
\hline d & 10 & $<20$ & $<20$ & $<20$ & $<20$ \\
\hline d & 30 & $26.90 \pm 2.15$ & $<20$ & $63.27 \pm 0.82$ & $<20$ \\
\hline ? & 10 & $<20$ & $<20$ & $<20$ & $<20$ \\
\hline $\mathrm{e}$ & 30 & $<20$ & $37.53 \pm 3.00$ & $33.94 \pm 0.75$ & $28.19 \pm 0.96$ \\
\hline$f$ & 10 & $<20$ & $29.13 \pm 1.59$ & $<20$ & $<20$ \\
\hline $\mathbf{I}$ & 30 & $26.61 \pm 0.57$ & $43.85 \pm 3.32$ & $38.45 \pm 1.93$ & $43.85 \pm 3.32$ \\
\hline 24 & & & & & \\
\hline 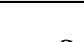 & 10 & $<20$ & $<20$ & $<20$ & $<20$ \\
\hline a & 30 & $89.01 \pm 0.47$ & $<20$ & $78.65 \pm 0.78$ & $46.21 \pm 1.54$ \\
\hline b & 10 & $<20$ & $<20$ & $<20$ & $<20$ \\
\hline D & 30 & $34.18 \pm 0.81$ & $<20$ & $31.07 \pm 2.36$ & $<20$ \\
\hline c & 10 & $<20$ & $<20$ & $<20$ & $<20$ \\
\hline $\mathrm{c}$ & 30 & $49.11 \pm 0.55$ & $<20$ & $43.22 \pm 1.52$ & $<20$ \\
\hline
\end{tabular}




\begin{tabular}{cc|cccc}
\hline \multirow{2}{*}{$\mathbf{d}$} & 10 & $<20$ & $<20$ & $<20$ & $<20$ \\
& 30 & $42.13 \pm 1.66$ & $<20$ & $55.41 \pm 0.76$ & $<20$ \\
\hline \multirow{2}{*}{$\mathbf{e}$} & 10 & $<20$ & $<20$ & $<20$ & $<20$ \\
& 30 & $83.66 \pm 0.34$ & $42.06 \pm 2.50$ & $70.11 \pm 1.06$ & $50.27 \pm 2.00$ \\
\hline \multirow{2}{*}{$\mathbf{f}$} & 10 & $<20$ & $<20$ & $22.34 \pm 2.06$ & $<20$ \\
& 30 & $84.77 \pm 1.18$ & $29.80 \pm 1.66$ & $68.27 \pm 1.19$ & $47.74 \pm 1.21$ \\
\hline \multirow{2}{*}{ cisplatin } & 10 & $42.61 \pm 2.33$ & $86.84 \pm 0.50$ & $53.03 \pm 2.29$ & $20.84 \pm 0.81$ \\
& 30 & $99.93 \pm 0.26$ & $90.18 \pm 1.78$ & $86.90 \pm 1.24$ & $74.47 \pm 1.20$ \\
& & {$[12.43]$} & {$[7.84]$} & {$[5.78]$} & {$[19.13]$}
\end{tabular}


Table 2

\begin{tabular}{|c|c|c|c|c|c|c|}
\hline & & $\begin{array}{r}\text { Grov } \\
{[\mathrm{c}}\end{array}$ & $\begin{array}{l}\text { Inhibition, } \% \\
\text { ulated } \mathrm{IC}_{50}(\mu\end{array}$ & & & \\
\hline & $\begin{array}{l}\text { Conc. } \\
(\mu \mathrm{M})\end{array}$ & $\mathrm{HeLa}$ & $\mathrm{SiHa}$ & MCF-7 & $\begin{array}{c}\text { MDA-MB- } \\
231\end{array}$ & NIH-3T3 \\
\hline 25 & & & & & & \\
\hline & 10 & $44.94 \pm 1.04$ & $21.17 \pm 2.05$ & $41.71 \pm 0.64$ & $47.32 \pm 1.15$ & $44.91 \pm 1.36$ \\
\hline $\mathbf{a}$ & 30 & $52.45 \pm 2.39$ & $66.23 \pm 0.86$ & $64.32 \pm 0.56$ & $71.49 \pm 0.75$ & $91.28 \pm 0.50$ \\
\hline & 10 & $51.49 \pm 3.62$ & $49.36 \pm 1.69$ & $44.58 \pm 1.50$ & $93.00 \pm 0.26$ & $44.81 \pm 1.50$ \\
\hline b & 30 & $62.58 \pm 2.21$ & $73.94 \pm 2.04$ & $50.52 \pm 3.26$ & $\begin{array}{c}93.71 \pm 0.09 \\
{[3.33]}\end{array}$ & $59.09 \pm 0.73$ \\
\hline & & $54.70 \pm 1.88$ & $49.58 \pm 2.11$ & $44.04 \pm 3.32$ & $77.13 \pm 1.07$ & \\
\hline c & $\begin{array}{l}10 \\
30\end{array}$ & $53.66 \pm 2.56$ & $61.83 \pm 2.77$ & $59.33 \pm 2.99$ & $\begin{array}{c}88.81 \pm 0.55 \\
{[5.91]}\end{array}$ & \\
\hline & 10 & $64.14 \pm 0.86$ & $70.88 \pm 1.03$ & $73.41 \pm 1.22$ & $95.04 \pm 0.16$ & $95.60 \pm 0.25$ \\
\hline d & 30 & $\begin{array}{c}90.12 \pm 0.99 \\
{[2.28]}\end{array}$ & $\begin{array}{c}94.14 \pm 0.29 \\
{[4.05]}\end{array}$ & $\begin{array}{c}80.16 \pm 3.40 \\
{[3.91]}\end{array}$ & $\begin{array}{c}95.60 \pm 0.06 \\
{[3.65]}\end{array}$ & $\begin{array}{c}98.22 \pm 0.04 \\
{[3.34]}\end{array}$ \\
\hline & 10 & $<20$ & $<20$ & $41.63 \pm 2.83$ & $21.96 \pm 0.73$ & \\
\hline e & 30 & $92.12 \pm 0.25$ & $89.25 \pm 0.68$ & $97.00 \pm 0.11$ & $95.22 \pm 0.91$ & \\
\hline & 10 & $45.08 \pm 0.72$ & $41.26 \pm 1.25$ & $55.41 \pm 1.26$ & $55.57 \pm 1.50$ & \\
\hline $\mathbf{f}$ & 30 & $39.39 \pm 0.49$ & $52.60 \pm 1.31$ & $62.52 \pm 0.67$ & $88.92 \pm 0.99$ & \\
\hline 26 & & & & & & \\
\hline & 10 & $37.98 \pm 2.68$ & $<20$ & $72.42 \pm 2.19$ & $46.43 \pm 2.05$ & $85.50 \pm 1.22$ \\
\hline $\mathbf{a}$ & 30 & $96.56 \pm 0.11$ & $96.71 \pm 0.17$ & $\begin{array}{c}98.72 \pm 0.09 \\
{[6.11]}\end{array}$ & $97.96 \pm 0.17$ & $\begin{array}{c}97.63 \pm 0.12 \\
{[5.97]}\end{array}$ \\
\hline b & 10 & $38.55 \pm 1.32$ & $<20$ & $31.80 \pm 1.35$ & $17.13 \pm 2.36$ & \\
\hline b & 30 & $43.97 \pm 2.23$ & $<20$ & $84.44 \pm 0.71$ & $37.72 \pm 2.28$ & \\
\hline & 10 & $36.30 \pm 1.45$ & $<20$ & $24.95 \pm 2.15$ & $<20$ & \\
\hline c & 30 & $35.53 \pm 1.24$ & $<20$ & $74.73 \pm 1.00$ & $<20$ & \\
\hline d & 10 & $<20$ & $<20$ & $47.25 \pm 1.78$ & $45.55 \pm 2.63$ & \\
\hline d & 30 & $22.15 \pm 1.29$ & $<20$ & $57.30 \pm 0.77$ & $59.79 \pm 1.22$ & \\
\hline & 10 & $<20$ & $<20$ & $68.51 \pm 0.71$ & $89.24 \pm 0.70$ & $31.41 \pm 2.21$ \\
\hline $\mathbf{e}$ & 30 & $96.98 \pm 0.33$ & $96.91 \pm 0.14$ & $\begin{array}{c}99.12 \pm 0.07 \\
{[6.53]}\end{array}$ & $\begin{array}{c}97.73 \pm 0.23 \\
{[5.69]}\end{array}$ & $\begin{array}{c}99.01 \pm 0.05 \\
{[11.75]}\end{array}$ \\
\hline & 10 & $21.62 \pm 3.46$ & $<20$ & $29.14 \pm 2.06$ & $40.46 \pm 2.98$ & $10.00 \pm 1.01$ \\
\hline $\mathbf{f}$ & 30 & $30.79 \pm 2.92$ & $27.28 \pm 1.90$ & $43.28 \pm 1.53$ & $76.93 \pm 1.60$ & $23.40 \pm 0.60$ \\
\hline 27 & & & & & & \\
\hline & 10 & $24.26 \pm 2.63$ & $34.00 \pm 1.43$ & $58.38 \pm 3.20$ & $56.24 \pm 0.98$ & $25.56 \pm 2.21$ \\
\hline $\mathbf{a}$ & 30 & $85.22 \pm 1.32$ & $82.68 \pm 1.25$ & $97.21 \pm 0.10$ & $84.18 \pm 0.44$ & $99.24 \pm 0.07$ \\
\hline & 10 & $37.10 \pm 1.77$ & $39.59 \pm 1.17$ & $51.92 \pm 1.00$ & $56.44 \pm 0.98$ & \\
\hline b & 30 & $52.08 \pm 2.08$ & $69.54 \pm 1.24$ & $65.12 \pm 1.91$ & $71.81 \pm 0.96$ & \\
\hline & 10 & $38.89 \pm 2.60$ & $64.05 \pm 1.24$ & $49.68 \pm 1.66$ & $72.37 \pm 1.27$ & $13.99 \pm 1.79$ \\
\hline c & 30 & $55.93 \pm 2.39$ & $\begin{array}{c}83.34 \pm 1.31 \\
{[9.29]}\end{array}$ & $61.26 \pm 1.72$ & $\begin{array}{c}85.81 \pm 1.04 \\
{[6.74]}\end{array}$ & $29.56 \pm 1.17$ \\
\hline & 10 & $34.23 \pm 1.39$ & $30.04 \pm 2.07$ & $47.03 \pm 1.25$ & $55.77 \pm 1.03$ & \\
\hline d & 30 & $47.74 \pm 0.78$ & $39.96 \pm 2.34$ & $42.43 \pm 1.69$ & $57.71 \pm 1.00$ & \\
\hline & 10 & $<20$ & $21.53 \pm 1.81$ & $35.74 \pm 1.33$ & $<20$ & \\
\hline e & 30 & $99.06 \pm 0.09$ & $96.91 \pm 0.06$ & $98.50 \pm 0.93$ & $99.01 \pm 0.52$ & \\
\hline & 10 & $<20$ & $24.65 \pm 1.46$ & $25.50 \pm 2.93$ & $24.79 \pm 2.20$ & \\
\hline f & 30 & $98.72 \pm 0.13$ & $96.04 \pm 0.25$ & $98.41 \pm 0.15$ & $98.79 \pm 0.16$ & \\
\hline
\end{tabular}




\begin{tabular}{cc|ccccc}
\hline \multirow{2}{*}{$\mathbf{a}$} & 10 & $35.48 \pm 1.91$ & $46.07 \pm 1.13$ & $52.88 \pm 0.82$ & $25.61 \pm 2.84$ & \\
& 30 & $63.44 \pm 1.79$ & $69.86 \pm 0.55$ & $73.39 \pm 0.74$ & $52.16 \pm 2.52$ & \\
\hline \multirow{2}{*}{$\mathbf{b}$} & 10 & $39.75 \pm 2.45$ & $<20$ & $43.51 \pm 1.85$ & $44.86 \pm 0.93$ & \\
& 30 & $47.34 \pm 1.62$ & $<20$ & $42.28 \pm 1.44$ & $43.73 \pm 2.25$ & \\
& 10 & $56.71 \pm 0.57$ & $39.93 \pm 3.14$ & $48.56 \pm 0.48$ & $30.30 \pm 1.64$ & \\
$\mathbf{c}$ & 30 & $58.21 \pm 0.73$ & $31.15 \pm 2.86$ & $49.93 \pm 1.33$ & $31.60 \pm 3.08$ & \\
& 10 & $74.18 \pm 1.15$ & $76.88 \pm 0.49$ & $75.97 \pm 0.89$ & $86.12 \pm 0.33$ & $70.18 \pm 1.15$ \\
\multirow{3}{*}{$\mathbf{d}$} & 30 & $91.17 \pm 0.33$ & $87.39 \pm 0.86$ & $88.99 \pm 0.25$ & $90.72 \pm 1.00$ & $91.12 \pm 1.64$ \\
& & {$[2.30]$} & {$[4.14]$} & {$[3.87]$} & {$[3.89]$} & {$[3.71]$} \\
\hline \multirow{2}{*}{$\mathbf{e}$} & 10 & $27.42 \pm 2.16$ & $<20$ & $52.86 \pm 1.30$ & $29.58 \pm 1.69$ & \\
& 30 & $92.94 \pm 0.17$ & $91.91 \pm 0.23$ & $96.38 \pm 0.07$ & $94.09 \pm 0.43$ & \\
& 10 & $30.97 \pm 1.02$ & $39.85 \pm 1.24$ & $50.60 \pm 0.65$ & $31.89 \pm 2.92$ & \\
$\mathbf{f}$ & 30 & $91.88 \pm 0.26$ & $90.94 \pm 0.18$ & $95.12 \pm 0.10$ & $92.56 \pm 0.34$ & \\
\hline \multirow{2}{*}{ cisplatin } & 10 & $42.61 \pm 2.33$ & $86.84 \pm 0.50$ & $53.03 \pm 2.29$ & $20.84 \pm 0.81$ & $94.20 \pm 0.39$ \\
& 30 & $99.93 \pm 0.26$ & $90.18 \pm 1.78$ & $86.90 \pm 1.24$ & $74.47 \pm 1.20$ & $96.44 \pm 0.17$ \\
& & {$[12.43]$} & {$[7.84]$} & {$[5.78]$} & {$[19.13]$} & {$[3.23]$} \\
\hline
\end{tabular}



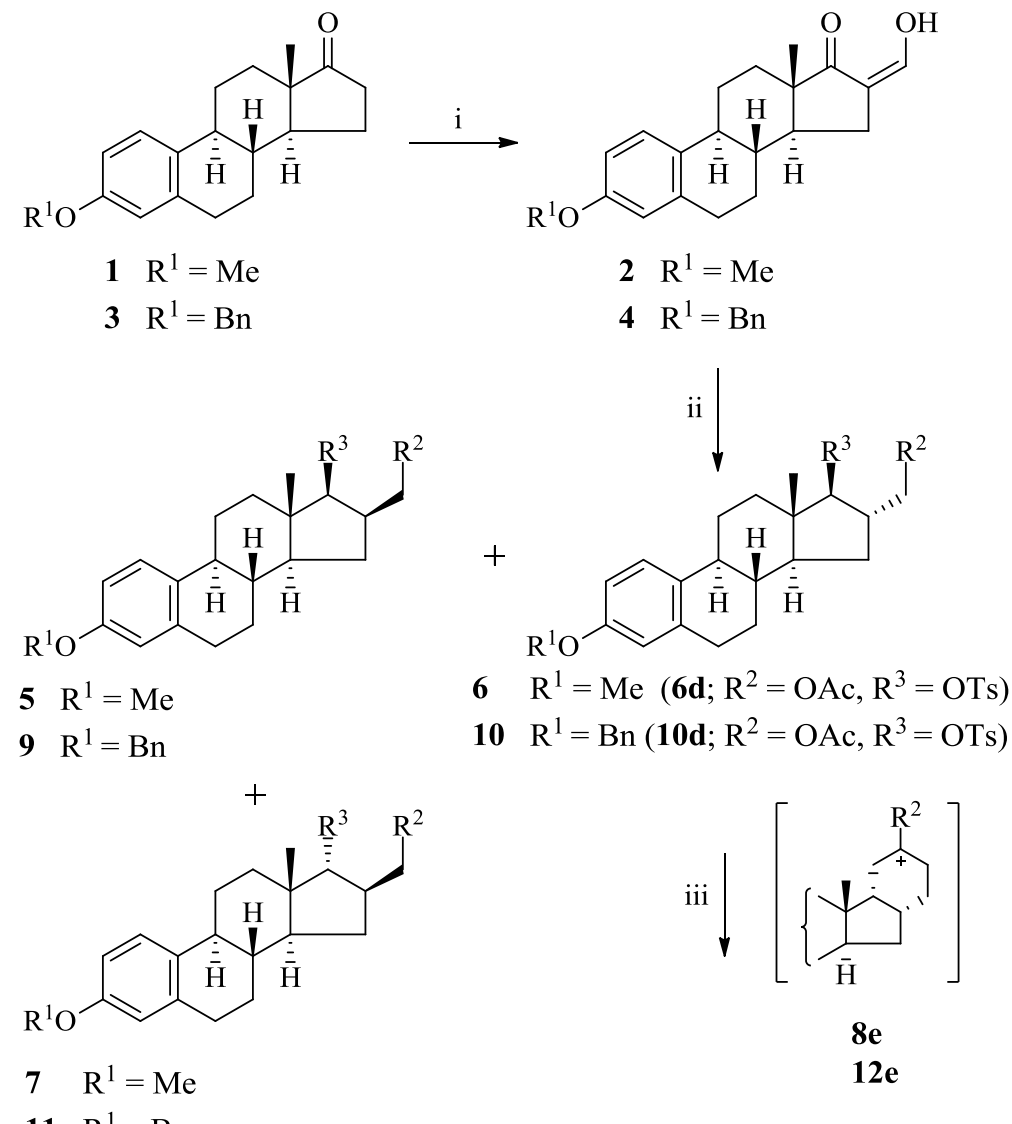

$11 \mathrm{R}^{1}=\mathrm{Bn}$

\begin{tabular}{l|l}
\hline $\mathbf{5 a}-\mathbf{1 2 a}$ & $\mathrm{R}^{2}=\mathrm{R}^{3}=\mathrm{OH}$ \\
$\mathbf{5 b}-\mathbf{8 b}$ & $\mathrm{R}^{2}=\mathrm{OTs}, \mathrm{R}^{3}=\mathrm{OH}$ \\
$\mathbf{9 c}-\mathbf{1 2 c}$ & $\mathrm{R}^{2}=\mathrm{Br}, \mathrm{R}^{3}=\mathrm{OH}$ \\
$\mathbf{6 d}, \mathbf{1 0 d}$ & $\mathrm{R}^{2}=\mathrm{OAc}, \mathrm{R}^{3}=\mathrm{OTs}$ \\
$\mathbf{8 e}, \mathbf{1 2 e}$ & $\mathrm{R}^{2}=\mathrm{Me}$
\end{tabular}

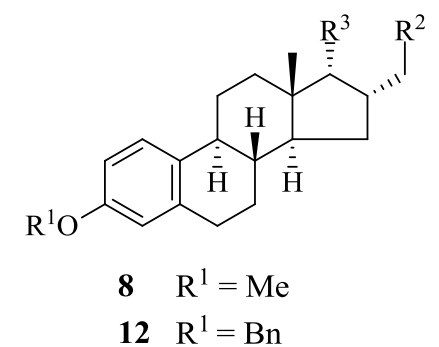

Scheme 1. Reagents and conditions: (i) NaOMe, HCOOEt, anhydrous toluene, $50{ }^{\circ} \mathrm{C}$; (ii) $\mathrm{KBH}_{4}, \mathrm{MeOH}$; (iii) $\mathrm{KOAc}, \mathrm{CH}_{3} \mathrm{COOH}, \mathrm{NaOMe} / \mathrm{MeOH}$

Scheme 1. 

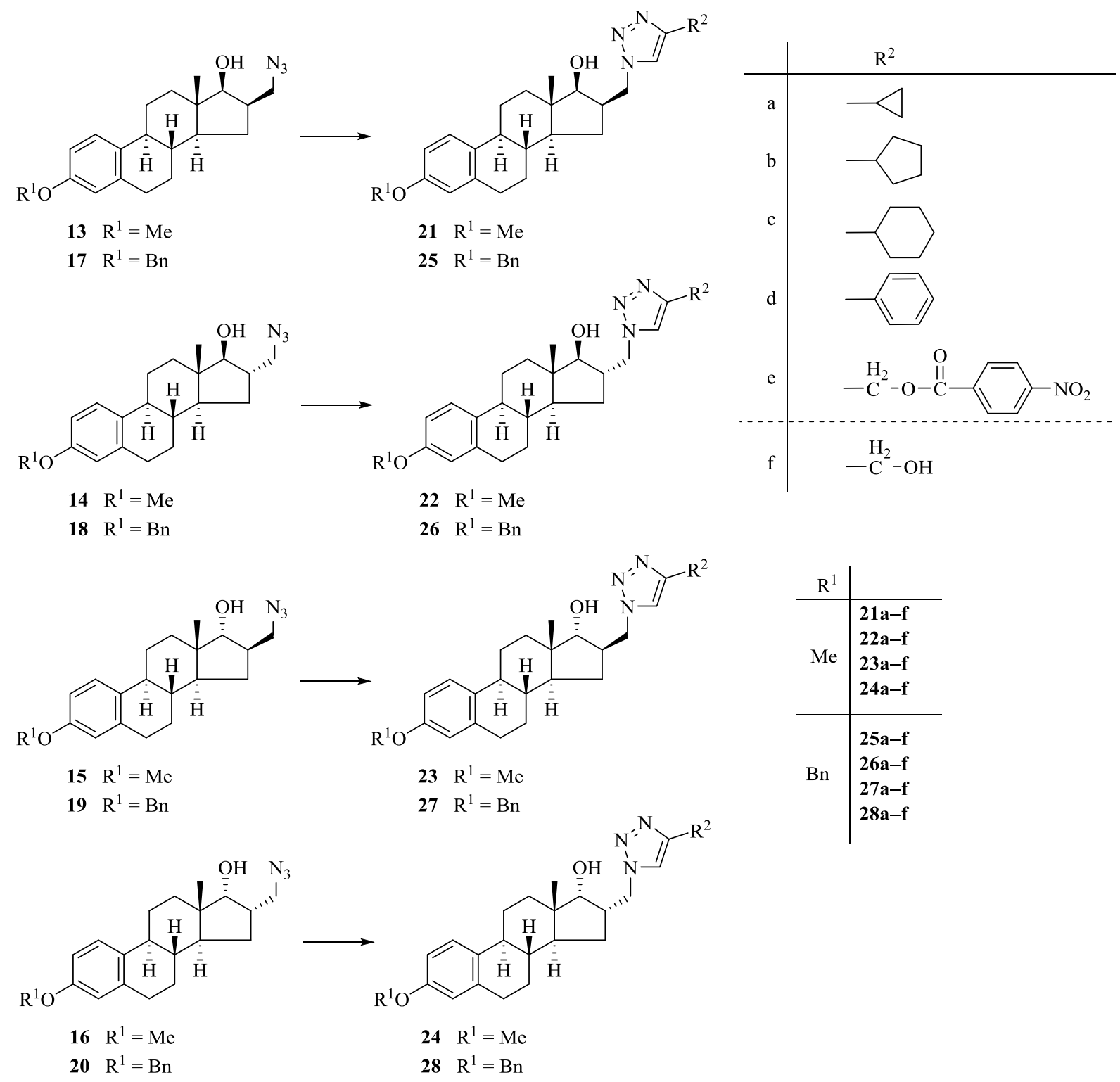

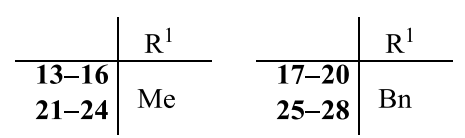

Scheme 2. 


\title{
Stereocontrolled Synthesis of the Four Possible 3-Methoxy and 3-Benzyloxy- 16-Triazolyl-methyl-estra-17-ol Hybrids and their Antiproliferative Activities
}

\author{
Anita Kiss ${ }^{a}$, János Wölfling ${ }^{a}$, Erzsébet Mernyák ${ }^{a}$, Éva Frank ${ }^{a}$, Zsanett Benke ${ }^{b}$, Seyyed \\ Ashkan Senobar Tahaei ${ }^{c}$, István Zupkóo ${ }^{\text {,d }}$, Sándor Mahó ${ }^{\mathrm{e}}$, Gyula Schneider $^{\text {** }}$ \\ aa Department of Organic Chemistry, University of Szeged, Dóm tér 8., H-6720 Szeged, Hungary \\ ${ }^{\mathrm{b}}$ Institute of Pharmaceutical Chemistry, University of Szeged, H-6720, Eötvös u. 6., H-6720 Szeged. Hungary \\ ${ }^{c}$ Department of Pharmacodynamics and Biopharmacy, University of Szeged, Eötvös u. 6., H-6720 Szeged, Hungary \\ ${ }^{\mathrm{d}}$ Interdisciplinary Centre for Natural Products, University of Szeged, Eötvös u. 6., H-6720 Szeged, Hungary \\ ${ }^{e}$ Chemical Works of Gedeon Richter Plc., Gyömröi út 19-21., H-1103 Budapest, Hungary
}

\begin{abstract}
The four possible isomers of each of 3-methoxy- and 3-benzyloxyestra-1,3,5(10)trien-17-ols (5-8 and 9-12) were converted through 16-p-tosyloxymethyl- or 16-bromomethyl derivatives into their 3-methoxy- and 3-benzyloxy-16-azidomethylestra(1,3,5(10)-triene derivatives (13-16 and 17-20). The regioselective $\mathrm{Cu}(\mathrm{I})$-catalyzed 1,3-dipolar cycloaddition of these compounds with different terminal alkynes afforded novel 1,4-disubstituted diastereomers (21a-f, 22a-f, 23a-f, 24a-f and 25a-f, 26a-f, 27a-f, 28a-f). The antiproliferative activities of the structurally related triazoles were determined in vitro with the microculture tetrazolium assay on four malignant human cell lines of gynecological origin (Hela, SiHa, MCF-7 and MDA-MB231).
\end{abstract}

Keywords: 3-methoxy- and 3-benzyloxy-16-azidomethylestra-1,3,5(10)-triene-17-ols; 1,3-dipolar cycloaddition, 4 'substituted-steroid triazoles; cytotoxic activity

\section{Introduction}


Among the hybrid natural products, hybrids of steroid frameworks have attracted great attention due to significant biological properties and numerous therapeutic effects of the basic compound. Steroids have become ideal synthons for the development of diverse conjugates due to their rigid framework and potential for varying levels of functionalization, broad biological activity profile and their ability to penetrate the cell membranes and bind to specific hormonal receptors [1-3].

The place, length and orientation of the linkers between the two parts of the hybrids stems unequivocally from the method of their synthesis. The literature provides a large number of methods to introduce the linker onto the sterane skeleton. The effect of the length and character of the linker are very often discussed [4]. However, only limited information is available with respect to the steric effect of the linkers on biological properties. As concerns the 16-substituted estrogenes, usually the $16 \alpha$-substituted-17 $\beta$-hydroxy compounds have been studied. The biological activity has generally not been studied for the whole isomer series [5].

In the 16-substituted 17-hydroxysteroids, the two chiral centres permit four stereochemical modifications. Since availability of the complete series of isomers would permit a number of interesting comparative examinations.

We have previously reported the preparation and configurational assignment of the four possible isomers of the 3-methoxy- and 3-benzyloxy-16-hydroxymethyl-estra-1,3,5(10)-trien-17ol derivatives (5a-8a and 9a-12a) [6-8]. Treatment of 3-methoxy- and 3-benzyloxyestra-16hydroxymethylidene-estra-1,3,5(10)-trien-17-ones (1 and 3) with $\mathrm{NaOMe}$ and ethyl formate gave 3-methoxy- and 3-benzyloxy-16-hydroxymethylidene-estra-1,3,5(10)-trien-17 ones (2 and 4). The C-16 formyl compounds were reduced with $\mathrm{KBH}_{4}$ in methanol yielding a mixture of three (5a-7a and 9a-11a) of the four possible isomers of each of the 3-methoxy- and 3-benzyloxy-16hydroxymethylestra-1,3,5(10)-trien-17-ol isomers in a ratio of 50:45:5 in 94\% yield [6,8 ]. The fourth isomers (8a and 12a) were prepared from 16 $\alpha$-acetoxymethyl-17 $\beta$-toluenesulfonate mixed esters $\mathbf{6 d}$ and 10d, respectively, by neighbouring group participation during solvolysis in aqueous AcOH. The structures of the isomers were confirmed unambiguously by their $\mathrm{IR},{ }^{1} \mathrm{H}$ and ${ }^{13} \mathrm{C}$ NMR spectra (Scheme 1) [7,8].

\section{(Scheme 1)}


The four 3-methoxy- and 3-benzyloxy-estra-1,3,5(10)-trien-17-ol isomers (5a-8a and 9a12a) are suitable starting materials to prepare 16-triazolyl-methyl derivatives. Triazoles are attractive units because of their stability against metabolic degradation and their ability to form hydrogen bonds. The $\mathrm{Cu}(\mathrm{I})$-catalysed azide-alkyne cycloaddition ( $\mathrm{CuAAC}$ ) is a facile method of wide applicability for the introduction of a triazole moiety into natural products [9]. In these compounds the triazole heterocycles and their substituted derivatives are connected through a methylene linker to the sterane skeleton. The 16-p-tolylsulfonyloxymethyl ester [5,6] and 16bromomethyl derivatives [10] of the 16-hydroxymethyl starting materials were used for substitution reaction with $\mathrm{NaN}_{3}$ in $N, N$-dimethylformamide to have the desired 3-methoxy- and 3-benzyloxy-16-azidomethylestra-1,3,5(10)-trien-17-ols (13-16 and 17-20). From these azido compounds several D-ring-substituted estrane derivatives containing a 1,2,3-triazole ring were synthesized by the reaction of 13-16 and 17-20 with various terminal alkynes through the use of the "click" chemistry approach to deliver compounds $21 \mathbf{a}-\mathbf{e}, 22 \mathbf{a}-\mathbf{e}, 23 \mathbf{a}-\mathbf{e}, 24 \mathbf{a}-\mathbf{e}, 25 \mathbf{a}-\mathbf{e}, 26 \mathbf{a}-\mathbf{e}$, $27 \mathbf{a}-\mathbf{e}$ and $28 \mathbf{a}-\mathbf{e}$.

\section{Experimental}

\subsection{General}

Melting points (Mp) were determined on a Kofler block and are uncorrected. Specific rotations were measured in $\mathrm{CHCl}_{3}\left(\begin{array}{c}c \\ 1)\end{array}\right)$ at $20{ }^{\circ} \mathrm{C}$ with a POLAMAT-A (Zeiss-Jena) polarimeter and are given in units of $10^{-1} \mathrm{deg} \mathrm{cm}^{2} \mathrm{~g}^{-1}$. Elementary analysis data were determined with a Perkin-Elmer CHN analyzer model 2400. The reactions were monitored by TLC on Kieselgel-G (Merck Si 254 F) layers (0.25 $\mathrm{mm}$ thick); solvent systems (ss): (A) diisopropyl ether, (B) acetone/toluene/hexane (30:35:35 v/v). The spots were detected by spraying with 5\% phosphomolybdic acid in $50 \%$ aqueous phosphoric acid. The $R_{\mathrm{f}}$ values were determined for the spots observed by illumination at 254 and $365 \mathrm{~nm}$. Flash chromatography: silica gel 60, 40-63 $\mu \mathrm{m}$. All solvents were distilled prior to use. NMR spectra were recorded on a Bruker DRX 500 and Bruker Ascend 500 instrument at $500\left({ }^{1} \mathrm{H}\right.$ NMR) or $125 \mathrm{MHz}\left({ }^{13} \mathrm{C}\right.$ NMR). Chemical shifts are reported in ppm $(\delta$ scale) and coupling constants $(J)$ in Hertz. For the determination of multiplicities, the $J$-MOD pulse sequence was used. 


\subsection{3-Methoxy- and 3-benzyloxy-16-azidomethylestra-1,3,5(10)-trienes (13-16 and 17-20) General procedure}

Compounds 5b-8b [5,6] (470 mg, $1 \mathrm{mmol})$ or 9c-12c [8] (455 mg, $1 \mathrm{mmol})$ were dissolved in $N, N$-dimethylformamide $(25 \mathrm{ml})$ and then $\mathrm{NaN}_{3}(260 \mathrm{mg})$ was added. The mixture was stirred for $6 \mathrm{~h}$ at $80{ }^{\circ} \mathrm{C}$, then poured into water $(50 \mathrm{ml})$. The precipitate separating out was filtered off and subjected to chromatographic separation with $\mathrm{CH}_{2} \mathrm{Cl}_{2} /$ hexane in different ratios.

\subsubsection{3-Methoxy-16 $\beta$-azidomethyl-estra-1,3,5(10)-trien-17 $\beta$-ol (13)}

Compound $\mathbf{5 b}$ (470 mg, $1 \mathrm{mmol}$ ) was used for the synthesis as described in Section 2.2. The crude product was chromatographed on silica gel with $\mathrm{CH}_{2} \mathrm{Cl}_{2} /$ hexane $(1: 3 \mathrm{v} / \mathrm{v})$ to yield pure $\mathbf{1 3}$ (318 mg, 93\%). Mp $134-135{ }^{\circ} \mathrm{C} ; R_{\mathrm{f}}=0.65(\mathrm{ss} \mathrm{A}) ;[\alpha]_{\mathrm{D}}{ }^{20}=+80\left(c 1\right.$ in $\left.\mathrm{CHCl}_{3}\right)$. (Found C, 70.23; $\mathrm{H}, 8.05 . \mathrm{C}_{20} \mathrm{H}_{27} \mathrm{~N}_{3} \mathrm{O}_{2}$ (341.45) requires $\left.\mathrm{C}, 70.35 ; \mathrm{H}, 7.97 \%\right) .{ }^{1} \mathrm{H}$ NMR $\left(\delta, \mathrm{ppm}, \mathrm{CDCl}_{3}\right): 0.82(\mathrm{~s}$, $\left.3 \mathrm{H}, 18-\mathrm{H}_{3}\right), 2.87\left(\mathrm{~m}, 2 \mathrm{H}, 6-\mathrm{H}_{2}\right), 3.32\left(\mathrm{dd}, 1 \mathrm{H}, J=12.5 \mathrm{~Hz}, J=7.5 \mathrm{~Hz}, 16 \mathrm{a}-\mathrm{H}_{2}\right), 3.61(\mathrm{dd}, 1 \mathrm{H}, J=$ $\left.12.5 \mathrm{~Hz}, J=7.5 \mathrm{~Hz}, 16 \mathrm{a}-\mathrm{H}_{2}\right), 3.78$ (s, 3H, 3- $\left.\mathrm{OCH}_{3}\right), 3.87$ (d, $\left.1 \mathrm{H}, J=10.0 \mathrm{~Hz}, 17-\mathrm{H}\right), 6.64$ (d, $1 \mathrm{H}$, $J=2.5 \mathrm{~Hz}, 4-\mathrm{H}), 6.72(\mathrm{dd}, 1 \mathrm{H}, J=8.5 \mathrm{~Hz}, J=2.5 \mathrm{~Hz}, 2-\mathrm{H}), 7.20(\mathrm{~d}, 1 \mathrm{H}, J=8.5 \mathrm{~Hz}, 1-\mathrm{H}) .{ }^{13} \mathrm{C}$ NMR ( $\delta, \mathrm{ppm}, \mathrm{CDCl}_{3}$ ): 12.2 (C-18), 26.3, 27.5, 29.7, 30.4, 37.7, 38.2, 40.2, 44.0, $44.3(\mathrm{C}-13)$, 49.0, 53.4 (C-16a), $55.2\left(3-\mathrm{OCH}_{3}\right), 81.5$ (C-17), 111.6 (C-2), 113.9 (C-4), 126.2 (C-1), 132.5 (C10), 137.9 (C-5), 157.7 (C-3).

\subsubsection{3-Methoxy-16 $\alpha$-azidomethylestra-1,3,5(10)-trien-17 $\beta$-ol (14)}

Compound $6 \mathbf{b}$ (470 mg, $1 \mathrm{mmol}$ ) was used for the synthesis as described in Section 2.2. The crude product was chromatographed on silica gel with $\mathrm{CH}_{2} \mathrm{Cl}_{2} /$ hexane $(1: 3 \mathrm{v} / \mathrm{v})$ to yield pure $\mathbf{1 4}$ (287 mg, 84\%). Mp 85-86 ${ }^{\circ} \mathrm{C} ; R_{\mathrm{f}}=0.62(\mathrm{ss} \mathrm{A}) ;[\alpha]_{\mathrm{D}}{ }^{20}=+48\left(c 1\right.$ in $\left.\mathrm{CHCl}_{3}\right)$. (Found C, 70.42; H, 7.65. $\mathrm{C}_{20} \mathrm{H}_{27} \mathrm{~N}_{3} \mathrm{O}_{2}(341.45)$ requires $\left.\mathrm{C}, 70.35 ; \mathrm{H}, 7.97 \%\right) .{ }^{1} \mathrm{H} \mathrm{NMR}\left(\delta, \mathrm{ppm}, \mathrm{CDCl}_{3}\right): 0.84(\mathrm{~s}, 3 \mathrm{H}$, $\left.18-\mathrm{H}_{3}\right), 2.86\left(\mathrm{~m}, 2 \mathrm{H}, 6-\mathrm{H}_{2}\right), 3.43(\mathrm{~d}, 1 \mathrm{H}, J=7.5 \mathrm{~Hz}, 17-\mathrm{H}), 3.48(\mathrm{dd}, 2 \mathrm{H}, J=6.5 \mathrm{~Hz}, \mathrm{~J}=3.5 \mathrm{~Hz}$, $\left.16 \mathrm{a}-\mathrm{H}_{2}\right), 3.78$ (s, 3H, 3-OCH $), 6.63(\mathrm{~s}, 1 \mathrm{H}, 4-\mathrm{H}), 6.72(\mathrm{dd}, 1 \mathrm{H}, J=6.5 \mathrm{~Hz}, J=2.0 \mathrm{~Hz}, 2-\mathrm{H}), 7.20$ $(\mathrm{d}, 1 \mathrm{H}, J=8.5 \mathrm{~Hz}, 1-\mathrm{H}) .{ }^{13} \mathrm{C}$ NMR $\left(\delta, \mathrm{ppm}, \mathrm{CDCl}_{3}\right): 11.8(\mathrm{C}-18), 26.1,27.2,28.0,29.7,36.6$, 38.5, 43.6, 43.9, 44.2 (C-13), 48.5, $55.2\left(3-\mathrm{OCH}_{3}\right), 55.6$ (C-16a), 85.1 (C-17), 111.5 (C-2), 113.8 (C-4), 126.3 (C-1), 132.4 (C-10), 137.8 (C-5), 157.5 (C-3). 


\subsubsection{3-Methoxy-16 $\beta$-azidomethylestra-1,3,5(10)-trien-17 $\alpha$-ol (15)}

Compound $7 \mathbf{b}$ (470 mg, $1 \mathrm{mmol}$ ) were used for the synthesis as described in Section 2.2. The crude porduct was chromatographed on silica gel with $\mathrm{CH}_{2} \mathrm{Cl}_{2} /$ hexane $(1: 3 \mathrm{v} / \mathrm{v})$ to yield pure 15 (275 mg, 80\%). Mp 96-98; ${ }^{\circ} \mathrm{C} ; R_{\mathrm{f}}=0.60$ (ss A); $[\alpha]_{\mathrm{D}}{ }^{20}=+68\left(c 1\right.$ in $\mathrm{CHCl}_{3}$ ). (Found C, 70.26; $\mathrm{H}, 8.15 . \mathrm{C}_{20} \mathrm{H}_{27} \mathrm{~N}_{3} \mathrm{O}_{2}$ (341.45) requires $\left.\mathrm{C}, 70.35 ; \mathrm{H}, 7.97 \%\right) .{ }^{1} \mathrm{H} \mathrm{NMR}\left(\delta, \mathrm{ppm}, \mathrm{CDCl}_{3}\right): 0.76$ (s, $\left.3 \mathrm{H}, 18-\mathrm{H}_{3}\right), 2.86\left(\mathrm{~m}, 2 \mathrm{H}, 6-\mathrm{H}_{2}\right), 3.43\left(\mathrm{dd}, 2 \mathrm{H}, J=7.5 \mathrm{~Hz}, \mathrm{~J}=3.0 \mathrm{~Hz}, 16 \mathrm{a}-\mathrm{H}_{2}\right), 3.61(\mathrm{~s}, 1 \mathrm{H}, 17-\mathrm{H})$, $3.78\left(\mathrm{~s}, 3 \mathrm{H}, 3-\mathrm{OCH}_{3}\right), 6.64(\mathrm{~d}, 1 \mathrm{H}, J=2.5 \mathrm{~Hz}, 4-\mathrm{H}), 6.72(\mathrm{dd}, 1 \mathrm{H}, J=8.5 \mathrm{~Hz}, J=2.5 \mathrm{~Hz}, 2-\mathrm{H})$,

$7.22(\mathrm{~d}, 1 \mathrm{H}, J=8.5 \mathrm{~Hz}, 1-\mathrm{H}) .{ }^{13} \mathrm{C} \mathrm{NMR}\left(\delta, \mathrm{ppm}, \mathrm{CDCl}_{3}\right): 17.7(\mathrm{C}-18), 25.9,27.9,29.8,30.3$, 31.9, 38.6, 43.3, 45.0 (C-13), 48.9, 55.2 (3- $\left.\mathrm{OCH}_{3}\right), 55.6$ (C-16a), 83.0 (C-17), 111.5 (C-2), 113.8 (C-4), 126.3 (C-1), 132.4 (C-10), 137.9 (C-5), 157.5 (C-3).

\subsubsection{3-Methoxy-16 $\alpha$-azidomethylestra-1,3,5(10)-trien-17 $\alpha$-ol (16)}

Compound $8 \mathbf{b}$ (470 mg, $1 \mathrm{mmol}$ ) was used for the synthesis as described in Section 2.2. The crude product was chromatographed on silica gel with $\mathrm{CH}_{2} \mathrm{Cl}_{2} /$ hexane $(1: 3 \mathrm{v} / \mathrm{v})$ to yield pure 16 (283 mg, 86\%). Mp 118-120 ${ }^{\circ} \mathrm{C} ; R_{\mathrm{f}}=0.65$ (ss A); $[\alpha]_{\mathrm{D}}{ }^{20}=+34\left(c 1\right.$ in $\left.\mathrm{CHCl}_{3}\right)$. (Found C, 70.55; $\mathrm{H}, 7.78 . \mathrm{C}_{20} \mathrm{H}_{27} \mathrm{~N}_{3} \mathrm{O}_{2}$ (341.45) requires $\mathrm{C}, 70.35 ; \mathrm{H}, 7.97 \%$ ). ${ }^{1} \mathrm{H} \mathrm{NMR}\left(\delta, \mathrm{ppm}, \mathrm{CDCl}_{3}\right.$ ): 0.80 (s, $\left.3 \mathrm{H}, 18-\mathrm{H}_{3}\right), 2.87\left(\mathrm{~m}, 2 \mathrm{H}, 6-\mathrm{H}_{2}\right), 3.35\left(\mathrm{dd}, 1 \mathrm{H}, J=12.0 \mathrm{~Hz}, J=6.0 \mathrm{~Hz}, 16 \mathrm{a}-\mathrm{H}_{2}\right), 3.53(\mathrm{dd}, 1 \mathrm{H}, J=$ $\left.12.0 \mathrm{~Hz}, J=9.5 \mathrm{~Hz}, 16 \mathrm{a}-\mathrm{H}_{2}\right), 3.78\left(\mathrm{~s}, 3 \mathrm{H}, 3-\mathrm{OCH}_{3}\right), 3.84(\mathrm{~d}, 1 \mathrm{H}, J=6.0 \mathrm{~Hz}, 17-\mathrm{H}), 6.63(\mathrm{~d}, 1 \mathrm{H}, J$ $=2.5 \mathrm{~Hz}, 4-\mathrm{H}), 6.72(\mathrm{dd}, 1 \mathrm{H}, J=8.5 \mathrm{~Hz}, 2-\mathrm{H}), 7.21(\mathrm{~d}, 1 \mathrm{H}, J=8.5 \mathrm{~Hz}, 1-\mathrm{H}) .{ }^{13} \mathrm{C} \mathrm{NMR}(\delta, \mathrm{ppm}$, $\mathrm{CDCl}_{3}$ ): 17.3 (C-18), 26.1, 28.0, 29.2, 31.3, 39.1, 40.5, 43.6, 46.4 (C-13), 47.0, 52.4 (C-16a), $55.2\left(3-\mathrm{OCH}_{3}\right), 79.9$ (C-17), 111.6 (C-2), 114.0 (C-4), 126.3 (C-1), 132.7 (C-10), 137.9 (C-5), $157.6(\mathrm{C}-3)$.

\subsubsection{3-Benzyloxy-16 $\beta$-azidomethylestra-1,3,5(10)-trien-17 $\beta$-ol (17)}

Compound 9c (455 mg, $1 \mathrm{mmol}$ ) was used for the synthesis as described in Section 2.2. The crude product was chromatographed on silica gel with $\mathrm{CH}_{2} \mathrm{Cl}_{2} /$ hexane $(1: 1 \mathrm{v} / \mathrm{v})$ to yield pure $\mathbf{1 7}$ (250 mg, 59\%). Mp 115-117 ${ }^{\circ} \mathrm{C} ; R_{\mathrm{f}}=0.45$ (ss A). (Found C, 74.55; H, 7.64. $\mathrm{C}_{26} \mathrm{H}_{31} \mathrm{~N}_{3} \mathrm{O}_{2}$ (417.54) requires $\mathrm{C}, 74.79 ; \mathrm{H}, 7.48 \%) .{ }^{1} \mathrm{H} \mathrm{NMR}\left(\delta, \mathrm{ppm}, \mathrm{CDCl}_{3}\right)$ : $0.82\left(\mathrm{~s}, 3 \mathrm{H}, 18-\mathrm{H}_{3}\right), 2.86(\mathrm{~m}$, $\left.2 \mathrm{H}, 6-\mathrm{H}_{2}\right), 3.33\left(\mathrm{dd}, 1 \mathrm{H}, J=12.0 \mathrm{~Hz}, J=7.5 \mathrm{~Hz}, 16 \mathrm{a}-\mathrm{H}_{2}\right), 3.60(\mathrm{dd}, 1 \mathrm{H}, J=12.5 \mathrm{~Hz}, J=7.5 \mathrm{~Hz}$, $\left.16 \mathrm{a}-\mathrm{H}_{2}\right), 3.87(\mathrm{~d}, 1 \mathrm{H}, J=9.5 \mathrm{~Hz}, 17-\mathrm{H}), 5.04\left(\mathrm{~s}, 2 \mathrm{H}, \mathrm{Bn}-\mathrm{H}_{2}\right), 6.73(\mathrm{~s}, 1 \mathrm{H}, 4-\mathrm{H}), 6.79(\mathrm{~d}, 1 \mathrm{H}, J=$ $8.0 \mathrm{~Hz}, J=2.0 \mathrm{~Hz}, 2-\mathrm{H}), 7.21(\mathrm{~d}, 1 \mathrm{H}, J=8.0 \mathrm{~Hz}, 1-\mathrm{H}), 7.32$ (t, $\left.1 \mathrm{H}, J=7.5 \mathrm{~Hz}, 4^{\prime}-\mathrm{H}\right), 7.39$ (t, 2H, 
$J=7.5 \mathrm{~Hz}, 3^{\prime}-\mathrm{H}$ and $\left.5^{\prime}-\mathrm{H}\right), 7.44\left(\mathrm{~d}, 2 \mathrm{H}, J=7.5 \mathrm{~Hz}, 2^{\prime}-\mathrm{H}\right.$ and $\left.6^{\prime}-\mathrm{H}\right) .{ }^{13} \mathrm{C}$ NMR $\left(\delta, \mathrm{ppm}, \mathrm{CDCl}_{3}\right)$ : 12.2 (C-18), 26.2, 27.5, 29.7, 30.3, 37.6, 38.1, 40.1, 43.9, 44.2 (C-13), 48.8 (C-16), 53.3 (C-16a), $69.9\left(\mathrm{Bn}^{-\mathrm{CH}_{2}}\right), 81.5$ (C-17), 112.3 (C-2), 114.8 (C-4), 126.3 (C-1), 127.3 (C-2' and C-6'), 127.8 (C-4'), 128.5 (C-3' and C-5'), 132.7 (C-10), 137.3 (C-1'), 137.9 (C-5), 156.8 (C-3).

\subsubsection{3-Benzyloxy-16 $\alpha$-azidomethylestra-1,3,5(10)-trien-17 $\beta$-ol (18)}

Compound 10c (455 mg, $1 \mathrm{mmol}$ ) was used for the synthesis as described in Section 2.2. The crude product was chromatographed on silica gel with $\mathrm{CH}_{2} \mathrm{Cl}_{2} /$ hexane $(3: 1 \mathrm{v} / \mathrm{v})$ to yield pure 18 (254 mg, 61\%). Mp 75-77 ${ }^{\circ} \mathrm{C} ; R_{\mathrm{f}}=0.40$ (ss A). (Found C, 74.87; H, 7.32. $\mathrm{C}_{26} \mathrm{H}_{31} \mathrm{~N}_{3} \mathrm{O}_{2}$ (417.54) requires $\mathrm{C}, 74.79 ; \mathrm{H}, 7.48 \%) .{ }^{1} \mathrm{H} \mathrm{NMR}\left(\delta, \mathrm{ppm}, \mathrm{CDCl}_{3}\right): 0.84$ (s, 3H, 18- $\left.\mathrm{H}_{3}\right), 2.85\left(\mathrm{~m}, 2 \mathrm{H}, 6-\mathrm{H}_{2}\right)$, $3.44(\mathrm{t}, 1 \mathrm{H}, J=8.0 \mathrm{~Hz}, 17-\mathrm{H}), 3.48\left(\mathrm{~m}, 2 \mathrm{H}, 16 \mathrm{a}-\mathrm{H}_{2}\right), 5.04\left(\mathrm{~s}, 2 \mathrm{H}, \mathrm{Bn}-\mathrm{H}_{2}\right), 6.73(\mathrm{~s}, 1 \mathrm{H}, 4-\mathrm{H}), 6.79$ $(\mathrm{d}, 1 \mathrm{H}, \mathrm{J}=8.5 \mathrm{~Hz}, 2-\mathrm{H}), 7.21(\mathrm{~d}, 1 \mathrm{H}, J=8.5 \mathrm{~Hz}, 1-\mathrm{H}), 7.32$ (t, $\left.1 \mathrm{H}, J=7.0 \mathrm{~Hz}, 4^{\prime}-\mathrm{H}\right), 7.39$ (t, 2H, $J=7.0 \mathrm{~Hz}, 3^{\prime}-$ and $\left.5^{\prime}-\mathrm{H}\right), 7.44\left(\mathrm{~d}, 2 \mathrm{H}, J=7.0 \mathrm{~Hz}, 2^{\prime}-\right.$ and $\left.6^{\prime}-\mathrm{H}\right) .{ }^{13} \mathrm{C}^{\mathrm{NMR}}\left(\delta, \mathrm{ppm}, \mathrm{CDCl}_{3}\right): 11.8$ (C-18), 26.1, 27.2, 27.9, 29.7, 36.6, 38.5, 43.6, 43.9, 44.2 (C-13), 48.6 (C-16), 55.6 (C-16a), 69.9 (Bn-CH $\left.{ }_{2}\right), 85.1$ (C-17), 112.3 (C-2), 114.8 (C-4), 126.3 (C-1), 127.4 (C-2' and -6'), 127.8 (C-4'), 128.5 (C-3' and -5'), 132.7 (C-10), 137.3 (C-1'), 137.9 (C-5), 156.8 (C-3).

\subsubsection{3-Benzyloxy-16 $\beta$-azidomethyl-estra-1,3,5(10)-trien-17 $\alpha$-ol (19)}

Copound 11c ( $455 \mathrm{mg}, 1 \mathrm{mmol}$ ) was used for the synthesis as described in Section 2.2. The crude product was chromatographed on silica gel with $\mathrm{CH}_{2} \mathrm{Cl}_{2} /$ hexane (3:1 v/v) to yield pure 19 (23. mg, 40\%). Mp. 134-136 ${ }^{\circ} \mathrm{C} . R_{\mathrm{f}}=0.38$ (ss A). (Found C, 74.92; H, 7.37. $\mathrm{C}_{26} \mathrm{H}_{31} \mathrm{~N}_{3} \mathrm{O}_{2}$ (417.54) requires $\mathrm{C}, 74.79 ; \mathrm{H}, 7.48 \%) .{ }^{1} \mathrm{H}$ NMR ( $\delta$, ppm, $\left.\mathrm{CDCl}_{3}\right)$ : 0.84 (s, 3H, 18- $\left.\mathrm{H}_{3}\right), 2.85\left(\mathrm{~m}, 2 \mathrm{H}, 6-\mathrm{H}_{2}\right)$, $3.43(\mathrm{~d}, 2 \mathrm{H}, J=8.0 \mathrm{~Hz}, 17-\mathrm{H}), 3.48$ (t, 2H, J = 6.5 Hz, 16a- $\left.\mathrm{H}_{2}\right), 5.04$ (s, 2H, Bn- $\left.\mathrm{H}_{2}\right), 6.73(\mathrm{~s}, 1 \mathrm{H}$, 4-H), 6.79 (d, 1H, $J=8.0 \mathrm{~Hz}, 2-\mathrm{H}), 7.22(\mathrm{~d}, 1 \mathrm{H}, J=8.0 \mathrm{~Hz} 1-\mathrm{H}), 7.33$ (d, 1H, $\left.J=7.0 \mathrm{~Hz}, 4^{\prime}-\mathrm{H}\right)$, 7.39 (t, $2 \mathrm{H}, J=7.0 \mathrm{~Hz}, 3^{\prime}-$ and $\left.5^{\prime}-\mathrm{H}\right), 7.44$ (d, $2 \mathrm{H}, J=7.0 \mathrm{~Hz}, 2^{\prime}-$ and $\left.6^{\prime}-\mathrm{H}\right) .{ }^{13} \mathrm{C}$ NMR ( $\delta$, ppm, $\mathrm{CDCl}_{3}$ ): 11.8 (C-18), 26.1, 27.2, 28.0, 29.7, 36.6, 38.4, 43.5, 43.9, 44.1 (C-13), 48.5 (C-16), 55.6 (C-16a), $69.9\left(\mathrm{Bn}^{-\mathrm{CH}_{2}}\right), 85.1$ (C-17), 112.3 (C-2), 114.8 (C-4), 126.3 (C-1), 127.4 (C-2' and -6'), 127.8 (C-4'), 128.5 (C-3' and -5'), 132.7 (C-10), 137.3 (C-1'), 137.9 (C-5), 156.7 (C-3).

\subsubsection{3-Benzyloxy-16 $\alpha$-azidomethyl-estra-1,3,5(10)-trien-17 $\alpha$-ol (20)}


Compound 12c (455 mg, $1 \mathrm{mmol}$ ) was used for the synthesis as described in Section 2.2. The crude was chromatographed on silica gel with $\mathrm{CH}_{2} \mathrm{Cl}_{2} /$ hexane $(1: 1 \mathrm{v} / \mathrm{v})$ to yield pure 20 (330 mg, $79 \%$ ). Mp 90-92 ${ }^{\circ} \mathrm{C} . R_{\mathrm{f}}=0.45$ (ss A). (Found C, 74.68; H, 7.55. $\mathrm{C}_{26} \mathrm{H}_{31} \mathrm{~N}_{3} \mathrm{O}_{2}$ (417.54) requires C, 74.79; H, 7.48\%). ${ }^{1} \mathrm{H}$ NMR ( $\delta, p p m, \mathrm{CDCl}_{3}$ ): 0.79 (s, 3H, 18- $\left.\mathrm{H}_{3}\right), 2.71$ (m, 2H, 6- $\mathrm{H}_{2}$ ), 3.35 (dd, $\left.1 \mathrm{H}, J=12.0 \mathrm{~Hz}, J=6.5 \mathrm{~Hz}, 16 \mathrm{a}-\mathrm{H}_{2}\right), 3.52\left(\mathrm{dd}, 1 \mathrm{H}, J=12.0 \mathrm{~Hz}, J=6.5 \mathrm{~Hz}, 16 \mathrm{a}-\mathrm{H}_{2}\right), 3.84(\mathrm{~d}, 1 \mathrm{H}$, $J=5.0 \mathrm{~Hz}, 17-\mathrm{H}), 5.04\left(\mathrm{~s}, 2 \mathrm{H}, \mathrm{Bn}-\mathrm{H}_{2}\right), 6.73(\mathrm{~s}, 1 \mathrm{H}, 4-\mathrm{H}), 6.79$ (dd, 1H, $J=8.5 \mathrm{~Hz}, J=2.5 \mathrm{~Hz}, 2-$ H), $7.22(\mathrm{~d}, 1 \mathrm{H}, J=8.5 \mathrm{~Hz}, 1-\mathrm{H}), 7.33$ (t, 1H, $\left.J=7.5 \mathrm{~Hz}, 4^{\prime}-\mathrm{H}\right), 7.39$ (t, 2H, $J=7.5 \mathrm{~Hz}, 3^{\prime}-$ and $\left.5^{\prime}-\mathrm{H}\right), 7.44\left(\mathrm{~d}, 2 \mathrm{H}, J=7.5 \mathrm{~Hz}, 2\right.$ ' - and 6'-H). ${ }^{13} \mathrm{C}$ NMR $\left(\delta, \mathrm{ppm}, \mathrm{CDCl}_{3}\right)$ : $17.2(\mathrm{C}-18), 26.0,27.9$, 29.0, 29.7, 31.2, 38.9, 40.4, 43.5, 46.3 (C-13), 46.8 (C-16), 52.2 (C-16a), 69.9 (Bn-CH $), 79.7$ (C17), 112.3 (C-2), 114.8 (C-4), 126.3 (C-1), 127.4 (C-2' and -6'), 127.8 (C-4'), 128.5 (C-3' and 5'), 132.8 (C-10), 137.3 (C-1'), 138.0 (C-5), 156.7 (C-3).

\subsection{General procedure for the synthesis of triazoles $(21 \boldsymbol{a}-\boldsymbol{e}, 22 \boldsymbol{a}-\boldsymbol{e}, 23 \boldsymbol{a}-\boldsymbol{e}, 24 \boldsymbol{a}-\boldsymbol{e}, 25 \boldsymbol{a}-\boldsymbol{e}, 26 \boldsymbol{a}-\boldsymbol{e}$,} $27 a-e$, and $28 a-e)$

3-Methoxy-16-azidomethylestra-1,3,5(10)-trien-17-ol isomers (13-16) (342 mg, $1 \mathrm{mmol}$ ) or 3benzyloxy-16-azidomethylestra-1,3,5(10)-trien-17-ol isomers (17-20) $418 \mathrm{mg}, 1 \mathrm{mmol}$ ) were dissolved in $\mathrm{CH}_{2} \mathrm{Cl}_{2}(20 \mathrm{ml})$, then $\mathrm{CuI}(19 \mathrm{mg}, 0.10 \mathrm{mmol}), \mathrm{Et}_{3} \mathrm{~N}(0.2 \mathrm{ml}, 2 \mathrm{mmol})$ and the appropriate terminal alkynes $(2 \mathrm{mmol})$ were added. The mixtures were stirred under reflux for 24 $\mathrm{h}$, then diluted with water $(30 \mathrm{ml})$ and extracted with $\mathrm{CH}_{2} \mathrm{Cl}_{2}(2 \times 30 \mathrm{ml})$. The combined organic phases were dried over $\mathrm{Na}_{2} \mathrm{SO}_{4}$ and evaporated in vacuo. The crude products were purified by flash chromatography using $\mathrm{CH}_{2} \mathrm{Cl}_{2}$ /ethyl acetate in different ratios.

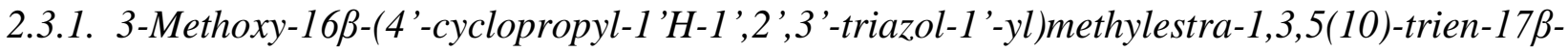 ol (21a)}

Compound 13 (342 mg, $1 \mathrm{mmol}$ ) and cyclopropylacetylene ( $2 \mathrm{mmol}, 0.22 \mathrm{ml}$ ) were used for the synthesis as described in Section 2.3. The crude product was chromatographed on silica gel with $\mathrm{CH}_{2} \mathrm{Cl}_{2} /$ hexane $(3: 1 \mathrm{v} / \mathrm{v})$ to yield pure $21 \mathrm{a}(210 \mathrm{mg}, 51 \%)$ as a white solid. $\mathrm{Mp}: 189-191{ }^{\circ} \mathrm{C} ; R_{\mathrm{f}}=$ 0.44 (ss B). (Found C, 73.84; H, 7.98. $\mathrm{C}_{25} \mathrm{H}_{33} \mathrm{~N}_{3} \mathrm{O}_{2}$ (407.55) requires $\mathrm{C}, 73.68 ; \mathrm{H}, 8.16 \%$ ). ${ }^{1} \mathrm{H}$ $\operatorname{NMR}\left(\delta, \mathrm{ppm}, \mathrm{CDCl}_{3}\right): 0.80\left(\mathrm{~s}, 3 \mathrm{H}, 18-\mathrm{H}_{3}\right), 0.83\left(\mathrm{~s}, 2 \mathrm{H}\right.$, cyclopropyl- $\left.\mathrm{H}_{2}\right), 0.94$ (s, 2H, cyclopropyl- $\left.\mathrm{H}_{2}\right), 2.72\left(\mathrm{~d}, 1 \mathrm{H}, J=7.0 \mathrm{~Hz}, 1\right.$ '”-H), $2.84\left(\mathrm{~m}, 2 \mathrm{H}, 6-\mathrm{H}_{2}\right), 3.77\left(\mathrm{~s}, 3 \mathrm{H}, 3-\mathrm{OCH}_{3}\right), 3.93$ $(\mathrm{d}, 1 \mathrm{H}, J=9.5 \mathrm{~Hz}, 17-\mathrm{H}), 4.21\left(\mathrm{dd}, 1 \mathrm{H}, J=13.0 \mathrm{~Hz}, J=6.0 \mathrm{~Hz}, 16 \mathrm{a}-\mathrm{H}_{2}\right), 4.62(\mathrm{t}, 1 \mathrm{H}, J=8.0 \mathrm{~Hz}$, 
16a- $\left.\mathrm{H}_{2}\right), 6.62(\mathrm{~s}, 1 \mathrm{H}, 4-\mathrm{H}), 6.71(\mathrm{~d}, 1 \mathrm{H}, J=8.5 \mathrm{~Hz}, 2-\mathrm{H}), 7.20(\mathrm{~d}, 1 \mathrm{H}, J=8.5 \mathrm{~Hz}, 1-\mathrm{H}), 7.29(\mathrm{~s}$, $\left.1 \mathrm{H}, 5^{\prime}-\mathrm{H}\right) .{ }^{13} \mathrm{C}$ NMR $\left(\delta, \mathrm{ppm}, \mathrm{CDCl}_{3}\right): 6.7$ (C-1"), 7.68 (C-2" and -3"),12.3 (C-18), 26.2, 27.4, 29.7, 30.8, 37.5, 38.0, 41.4, 43.8, 44.3 (C-16a), 48.7, 51.7 (C-13), $55.2\left(3-\mathrm{OCH}_{3}\right), 80.7$ (C-17), 111.5 (C-2), 113.8 (C-4), 126.3 (C-1), 132.4 (C-10), 137.8 (C-5), 157.5 (C-3).

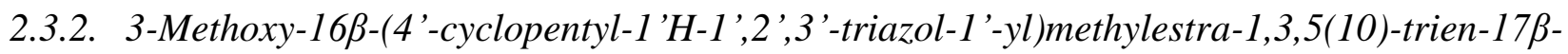
ol (21b)

Compound 13 (342 mg, $1 \mathrm{mmol}$ ) and cyclopentylacetylene (2 mmol, $0.22 \mathrm{ml})$ were used for the synthesis as described in Section 2.3. The crude product was chromatographed on silica gel with $\mathrm{CH}_{2} \mathrm{Cl}_{2}$ to yield pure $21 \mathrm{~b}$ (370 $\mathrm{mg}, 85 \%$ ) as a white solid. Mp: $191-192{ }^{\circ} \mathrm{C} ; R_{\mathrm{f}}=0.46$ (ss B). (Found C, 74.62; H, 8.42. $\mathrm{C}_{27} \mathrm{H}_{37} \mathrm{~N}_{3} \mathrm{O}_{2}$ (435.60) requires $\left.\mathrm{C}, 74.45 ; \mathrm{H}, 8.56 \%\right) .{ }^{1} \mathrm{H}$ NMR ( $\delta$, ppm, $\mathrm{CDCl}_{3}$ ): 0.79 (s, 3H, 18- $\left.\mathrm{H}_{3}\right), 2.85\left(\mathrm{~m}, 2 \mathrm{H}, 6-\mathrm{H}_{2}\right), 3.19(\mathrm{~s}, 1 \mathrm{H}, 1$ ”- $\mathrm{H}), 3.77\left(\mathrm{~s}, 3 \mathrm{H}, 3-\mathrm{OCH}_{3}\right), 3.94$ $(\mathrm{d}, 1 \mathrm{H}, J=9.5 \mathrm{~Hz}, 17-\mathrm{H}), 4.24\left(\mathrm{~d}, 1 \mathrm{H}, J=8.0 \mathrm{~Hz}, 16 \mathrm{a}-\mathrm{H}_{2}\right), 4.65\left(\mathrm{~s}, 1 \mathrm{H}, 16 \mathrm{a}-\mathrm{H}_{2}\right), 6.62(\mathrm{~s}, 1 \mathrm{H}, 4-$ $\mathrm{H}), 6.71(\mathrm{~d}, 1 \mathrm{H}, J=8.5 \mathrm{~Hz}, 2-\mathrm{H}), 7.20(\mathrm{~d}, 1 \mathrm{H}, J=8.5 \mathrm{~Hz}, 1-\mathrm{H}), 7.34$ (s, $1 \mathrm{H}, 5$ '-H). ${ }^{13} \mathrm{C} \mathrm{NMR}(\delta$, ppm, $\mathrm{CDCl}_{3}$ ): 12.3 (C-18), 25.1 (C-3" and -4"), 26.2, 27.4, 29.7 (C-2" and 5"), 30.8, 33.2, 36.7, 37.5, 38.0, 42.4 (C-16a), 43.8, 44.3 (C-13), 48.7, 51.8, 55.2 (3-OCH 3 ), 62.1 (C-16), 80.7 (C-17), 111.5 (C-2), 113.7 (C-4), 126.3 (C-1), 132.4 (C-10), 137.8 (C-5), 157.4 (C-3).

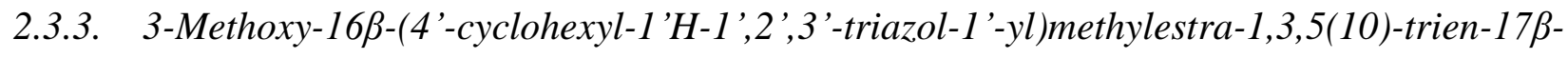
ol (21c)

Compound 13 (342 mg, $1 \mathrm{mmol}$ ) and cyclohexylacetylene ( $2 \mathrm{mmol}, 0.22 \mathrm{ml})$ were used for the synthesis as described in Section 2.3. The crude product was chromatographed on silica gel with ethyl acetate/ $\mathrm{CH}_{2} \mathrm{Cl}_{2}(1: 99 \mathrm{v} / \mathrm{v}$ ) to yield pure 21c (370 mg, 82\%) as a white solid. Mp: 189-190 ${ }^{\circ} \mathrm{C} ; R_{\mathrm{f}}=0,40$ (ss B). (Found $\mathrm{C}, 74.92 ; \mathrm{H}, 8.55 . \mathrm{C}_{28} \mathrm{H}_{41} \mathrm{~N}_{3} \mathrm{O}_{2}$ (449.63) requires $\mathrm{C}, 74.80 ; \mathrm{H}, 8.74 \%$ ). ${ }^{1} \mathrm{H}$ NMR ( $\delta$, ppm, $\mathrm{CDCl}_{3}$ ): 0.79 (s, 3H, 18- $\mathrm{H}_{3}$ ), 2.84 (m, 2H, 6- $\mathrm{H}_{2}$ ), 3.77 (s, 3H, 3- $-\mathrm{OCH}_{3}$ ), 3.94 $(\mathrm{d}, 1 \mathrm{H}, J=9.5 \mathrm{~Hz}, 17-\mathrm{H}), 4.24\left(\mathrm{~m}, 1 \mathrm{H}, 16 \mathrm{a}-\mathrm{H}_{2}\right), 4.65\left(\mathrm{~m}, 1 \mathrm{H}, 16 \mathrm{a}-\mathrm{H}_{2}\right), 6.62(\mathrm{~s}, 1 \mathrm{H}, 4-\mathrm{H}), 6.71(\mathrm{~d}$, $1 \mathrm{H}, J=8.5 \mathrm{~Hz}, 2-\mathrm{H}), 7.20(\mathrm{~d}, 1 \mathrm{H}, J=8.5 \mathrm{~Hz}, 1-\mathrm{H}), 7.32\left(\mathrm{~s}, 1 \mathrm{H}, 5{ }^{\prime}-\mathrm{H}\right) .{ }^{13} \mathrm{C}$ NMR $(\delta, \mathrm{ppm}$, $\mathrm{CDCl}_{3}$ ): 12.3 (C-18), 26.0, 26.1 (C-2" and -6"), 26.2, 27.4, 29.7, 30.8, 33.0, 37.5, 38.0, 41.4 (C1”), 43.8, 44.3 (C-13), 48.3, $55.2\left(3-\mathrm{OCH}_{3}\right), 62.1,80.7$ (C-17), 111.5 (C-2), 113.7 (C-4), 126.3 (C-1), 132.4 (C-10), 137.8 (C-5), $157.4(\mathrm{C}-3)$. 


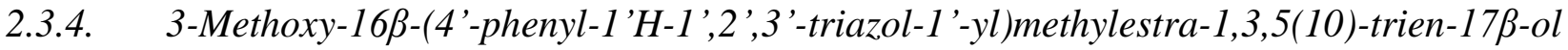

(21d)

Compound $13(342 \mathrm{mg}, 1 \mathrm{mmol})$ and phenylacetylene $(2 \mathrm{mmol}, 0.22 \mathrm{ml})$ were used for the synthesis as described in Section 2.3. The crude product was chromatographed on silica gel with ethyl acetate/ $\mathrm{CH}_{2} \mathrm{Cl}_{2}(1: 99 \mathrm{v} / \mathrm{v})$ to yield pure 21d (368 mg, 83\%) as a white solid. Mp: 232-234 ${ }^{\circ} \mathrm{C} ; R_{\mathrm{f}}=0.35$ (ss B). (Found C, 75.98; H, 7.36. $\mathrm{C}_{28} \mathrm{H}_{33} \mathrm{~N}_{3} \mathrm{O}_{2}(443.58$ ) requires $\mathrm{C}, 75.81 ; \mathrm{H}, 7.50 \%$ ). ${ }^{1} \mathrm{H}$ NMR ( $\delta$, ppm, $\mathrm{CDCl}_{3}$ ): 0.79 (s, 3H, 18- $\mathrm{H}_{3}$ ), 2.73 (m, 2H, 6- $\mathrm{H}_{2}$ ), 3.68 (s, 3H, 3- $\left.\mathrm{OCH}_{3}\right), 3.79$ $(\mathrm{d}, 1 \mathrm{H}, J=10.0 \mathrm{~Hz}, 17-\mathrm{H}), 4.20\left(\mathrm{t}, 1 \mathrm{H}, J=13.5 \mathrm{~Hz}, 16 \mathrm{a}-\mathrm{H}_{2}\right), 4.63$ (dd, $1 \mathrm{H}, J=13.5 \mathrm{~Hz}, J=4.5$ Hz, 16a- $\left.\mathrm{H}_{2}\right), 6.59$ (s, 1H, 4-H), 6.67 (d, 1H, $\left.J=8.5 \mathrm{~Hz}, 2-\mathrm{H}\right), 7.16$ (d, 1H, $\left.J=8.5 \mathrm{~Hz}, 1-\mathrm{H}\right), 7.32$ (t, $1 \mathrm{H}, J=7.5 \mathrm{~Hz}, 4$ "'-H), 7.44 (t, 2H, $J=7.5 \mathrm{~Hz}, 3$ "'- and 5"-H), 7.85 (d, 2H, $J=7.5 \mathrm{~Hz}, 2$ "'- and 6"-H), 8.60 (s, $1 \mathrm{H}, 5$ '-H). ${ }^{13} \mathrm{C}$ NMR ( $\delta$, ppm, $\mathrm{CDCl}_{3}$ ): 12.4 (C-18), 25.8, 26.9, 29.1, 30.0, 36.9, 37.8, 40.4, 43.3, 43.7 (C-13), 47.8, 52.3 (C-16a), 54.8 (3-- $\left.\mathrm{OCH}_{3}\right), 79.5$ (C-17), 111.4 (C-2), 113.3 (C-4), 121.5 (C-5'), 124.5 (C-2" and -6”), 126.0 (C-1), 127.6 (C-4"), 127.8 (C-3" and -5"), 130.9 (C-1”), 132.0 (C-10), 137.3 (C-5), 146.0 (C-4'), 156.9 (C-3).

2.3.5. 3-Methoxy-16ß-(4'-nitro-benzoyloxymethyl-1 'H-1',2, '3'-triazol-1'-yl)methylestra1,3,5(10)-trien-17 $\beta$-ol (21e)

Compound 13 (342 mg, $1 \mathrm{mmol}$ ) and propargyl 4-nitrobenzoate ( $2 \mathrm{mmol}, 410 \mathrm{mg}$ ) were used for the synthesis as described in Section 2.3. The crude product was chromatographed on silica gel with ethyl acetate/ $\mathrm{CH}_{2} \mathrm{Cl}_{2}(5: 95 \mathrm{v} / \mathrm{v})$ to yield pure $21 \mathrm{e}(475 \mathrm{mg}, 86 \%)$ as a yellow solid. Mp: 134-135.5 ${ }^{\circ} \mathrm{C} ; R_{\mathrm{f}}=30$ (ss B). (Found C, 66.12; H, 6.08. $\mathrm{C}_{30} \mathrm{H}_{34} \mathrm{~N}_{4} \mathrm{O}_{6}(546.61$ ) requires C, 65.92; $\mathrm{H}, 6.27 \%) .{ }^{1} \mathrm{H} \operatorname{NMR}\left(\delta, \mathrm{ppm}, \mathrm{CDCl}_{3}\right): 0.73$ (s, 3H, 18- $\left.\mathrm{H}_{3}\right), 2.70\left(\mathrm{~m}, 2 \mathrm{H}, 6-\mathrm{H}_{2}\right), 3.66$ (s, 3H, 3$\left.\mathrm{OCH}_{3}\right), 4.18(\mathrm{dd}, 1 \mathrm{H}, J=13.5 \mathrm{~Hz}, J=11.5 \mathrm{~Hz}, 16 \mathrm{a}-\mathrm{H} 2), 4.58(\mathrm{dd}, 1 \mathrm{H}, J=13.5 \mathrm{~Hz}, J=4.5 \mathrm{~Hz}$, 16a- $\left.\mathrm{H}_{2}\right), 5.02$ (d, 1H, $\left.J=4.5 \mathrm{~Hz}, 17-\mathrm{H}\right), 5.44\left(\mathrm{~s}, 2 \mathrm{H}, 4^{\prime}-\mathrm{H}_{2}\right), 6.55$ (d, 1H, $\left.J=1.5 \mathrm{~Hz}, 4-\mathrm{H}\right), 6.63$ $(\mathrm{dd}, 1 \mathrm{H}, J=8.5 \mathrm{~Hz}, J=2.0 \mathrm{~Hz}, 2-\mathrm{H}), 7.12(\mathrm{~d}, 1 \mathrm{H}, J=8.5 \mathrm{~Hz}, 1-\mathrm{H}), 8.16(\mathrm{~d}, 2 \mathrm{H}, J=8.5 \mathrm{~Hz}, 3$ ”and 5 "- $\mathrm{H}), 8.31$ (t, 3H, $J=8.5 \mathrm{~Hz}, 2$ "- and 6 "-H, 5 '-H) ${ }^{13} \mathrm{C}$ NMR $\left(\delta, \mathrm{ppm}, \mathrm{CDCl}_{3}\right): 12.3(\mathrm{C}-18)$, 25.8, 26.9, 29.1, 30.0, 36.9, 37.8, 40.4, 43.3, 43.7 (C-13), 47.8, 52.2 (C-16a), $54.7\left(3-\mathrm{OCH}_{3}\right)$, 58.7 (4'- $\mathrm{CH}_{2}$ ), 79.5 (C-17), 111.3 (C-2), 113.3 (C-4), 123.8 (C-2"' and -6"), 125.1 (C-5'), 126.0 (C-1), 130.6 (C-3" and -5"), 131.9 (C-10), 134.7 (C-1"), 137.2 (C-5), 141.0 (C-4"), 150.2 (C-4'), $156.9(\mathrm{C}-3), 163.9(\mathrm{C}=\mathrm{O})$. 


\subsubsection{3-Methoxy-16ß-(4'-hydroxymethyl-1 'H-1 ',2',3'-triazol-1'-yl)methylestra-1,3,5(10)-trien-}

$17 \beta-o l(21 f)$

Compound 13 (274 mg, $0.5 \mathrm{mmol})$ was dissolved in methanol (10 ml) containing $\mathrm{NaOCH}_{3}(14$ $\mathrm{mg}, 0.25 \mathrm{mmol}$ ), and the solution was allowed to stand for $24 \mathrm{~h}$. It was then diluted with water, and the precipitate separating out was filtered off and recrystallized from a mixture of ethyl acetate/hexane to afford $\mathbf{2 1 f}(171 \mathrm{mg}, 86 \%)$ as a white crystalline material. Mp: $194-195{ }^{\circ} \mathrm{C} ; R_{\mathrm{f}}=$ 0.25 (ss B). (Found C, 69.23; H, 8.04. $\mathrm{C}_{23} \mathrm{H}_{31} \mathrm{~N}_{3} \mathrm{O}_{3}$ (397.51) requires C, 69.49; H, 7.86\%). ${ }^{1} \mathrm{H}$ NMR ( $\delta$, ppm, DMSO-d $\left.{ }_{6}\right): 0.76\left(\mathrm{~s}, 3 \mathrm{H}, 18-\mathrm{H}_{3}\right), 2.71\left(\mathrm{~m}, 2 \mathrm{H}, 6-\mathrm{H}_{2}\right), 3.68\left(\mathrm{~s}, 3 \mathrm{H}, 3-\mathrm{OCH}_{3}\right), 3.76$ (d, $1 \mathrm{H}, J=5.5 \mathrm{~Hz}, 17-\mathrm{H}), 4.14\left(\mathrm{t}, 1 \mathrm{H}, J=12.5 \mathrm{~Hz}, 16 \mathrm{a}-\mathrm{H}_{2}\right), 4.49\left(\mathrm{~m}, 3 \mathrm{H}, 4^{\prime}-\mathrm{H}_{2}\right.$ and $\left.16 \mathrm{a}-\mathrm{H}_{2}\right)$, $5.03(\mathrm{~d}, 1 \mathrm{H}, J=3.5 \mathrm{~Hz}, 17-\mathrm{OH}), 5.15$ (brs, $1 \mathrm{H}, \mathrm{CH}_{2}-\mathrm{OH}$ ), 6.59 (s, $\left.1 \mathrm{H}, 4-\mathrm{H}\right), 6.66$ (d, $1 \mathrm{H}, J=8.5$ $\mathrm{Hz}, 2-\mathrm{H}), 7.16(\mathrm{~d}, 1 \mathrm{H}, J=8.5 \mathrm{~Hz}, 1-\mathrm{H}), 7.99$ (s, $\left.1 \mathrm{H}, 5^{\prime}-\mathrm{H}\right) .{ }^{13} \mathrm{C}$ NMR ( $\delta$, ppm, DMSO-d $\left.\mathrm{d}_{6}\right): 12.4$ (C-18), 25.9, 26.9, 29.2, 30.0, 36.9, 37.9, 40.5, 43.4, 43.8 (C-13), 47.8, 52.0 (C-16a), 54.8 (3$\left.\mathrm{OCH}_{3}\right), 55.0$ (4'- $\left.\mathrm{CH}_{2}\right), 79.5$ (C-17), $111.4(\mathrm{C}-2), 113.4$ (C-4), 122.8 (C-5'), 126.1 (C-1), 132.0 (C-10), 137.3 (C-5), 147.6 (C-4'), 157.0 (C-3).

\subsubsection{3-Methoxy-16a-(4'-cyclopropyl-1 'H-1',2',3'-triazol-1'-yl)methylestra-1,3,5(10)-trien-17ß- ol (22a)}

Compound 14 (342 mg, $1 \mathrm{mmol})$ and cyclopropylacetylene $(2 \mathrm{mmol}, 0.22 \mathrm{ml})$ were used for the synthesis as described in Section 2.3. The crude product was chromatographed on silica gel with ethyl acetate/ $\mathrm{CH}_{2} \mathrm{Cl}_{2}(5: 95 \mathrm{v} / \mathrm{v})$ to yield pure 22a $(261 \mathrm{mg}, 64 \%)$ as a white solid. Mp: $67-69{ }^{\circ} \mathrm{C}$; $R_{\mathrm{f}}=0.35$ (ss B). (Found C, 73.55; H, 7.98. $\mathrm{C}_{25} \mathrm{H}_{33} \mathrm{~N}_{3} \mathrm{O}_{2}$ (407.55) requires C, 73.68; H, 8.16\%). ${ }^{1} \mathrm{H}$ $\operatorname{NMR}\left(\delta, \mathrm{ppm}, \mathrm{CDCl}_{3}\right): 0.82\left(\mathrm{~m}, 5 \mathrm{H}, 18-\mathrm{H}_{3}\right.$ and cyclopropyl- $\left.\mathrm{H}_{2}\right), 0.95\left(\mathrm{~m}, 2 \mathrm{H}\right.$, cyclopropyl- $\left.\mathrm{H}_{2}\right)$, $2.83\left(\mathrm{~m}, 2 \mathrm{H}, 6-\mathrm{H}_{2}\right), 3.53$ (d, $\left.1 \mathrm{H}, J=7.5 \mathrm{~Hz}, 17-\mathrm{H}\right), 3.77$ (s, $\left.3 \mathrm{H}, 3-\mathrm{OCH}_{3}\right), 4.35$ (t, $1 \mathrm{H}, J=7.5 \mathrm{~Hz}$, $\left.16 \mathrm{a}-\mathrm{H}_{2}\right), 4.44\left(\mathrm{dd}, 1 \mathrm{H}, J=13.5 \mathrm{~Hz}, J=7.5 \mathrm{~Hz}, 16 \mathrm{a}-\mathrm{H}_{2}\right), 6.62(\mathrm{~d}, 1 \mathrm{H}, J=2.0 \mathrm{~Hz}, 4-\mathrm{H}), 6.70(\mathrm{dd}$, $1 \mathrm{H}, J=8.5 \mathrm{~Hz}, J=2.0 \mathrm{~Hz}, 2-\mathrm{H}), 7.18(\mathrm{~d}, 1 \mathrm{H}, J=8.5 \mathrm{~Hz}, 1-\mathrm{H}) .{ }^{13} \mathrm{C}$ NMR $\left(\delta, \mathrm{ppm}, \mathrm{CDCl}_{3}\right): 6.7$ (C-1"), 7.7 (C-2" and -3"), 11.8 (C-18), 26.1, 27.2, 28.2, 29.7, 36.6, 38.4, 43.9, 44.3, 44,3 (C16a), 48.3, 54.5 (C-13), $62.1\left(3-\mathrm{OCH}_{3}\right), 85.1$ (C-17), 111.5 (C-2), 113.8 (C-4), 126.2 (C-1), 132.3 (C-10), 137.8 (C-5), 157.4 (C-3).

2.3.8. 3-Methoxy-16a-(4'-cyclopentyl-1 'H-1',2',3'-triazol-1-yl)methylestra-1,3,5(10)-trien-17ßol $(22 b)$ 
Compound 14 (342 mg, $1 \mathrm{mmol})$ and cyclopentylacetylene (2 mmol, $0.22 \mathrm{ml})$ were used for the synthesis as described in Section 2.3. The crude product was chromatographed on silica gel with ethyl acetate $/ \mathrm{CH}_{2} \mathrm{Cl}_{2}(5: 95 \mathrm{v} / \mathrm{v})$ to yield pure 22b $(290 \mathrm{mg}, 66 \%)$ as a white solid. Mp: 163-165 ${ }^{\circ} \mathrm{C} ; R_{\mathrm{f}}=0.32$ (ss B). (Found C, 74.63; H, 8.41. $\mathrm{C}_{27} \mathrm{H}_{37} \mathrm{~N}_{3} \mathrm{O}_{2}$ (435.60) requires $\mathrm{C}, 74.45 ; \mathrm{H}, 8.56 \%$ ). ${ }^{1} \mathrm{H}$ NMR $\left(\delta, \mathrm{ppm}, \mathrm{CDCl}_{3}\right): 0.83\left(\mathrm{~s}, 3 \mathrm{H}, 18-\mathrm{H}_{3}\right), 1.68$ (s, 4H, 3"'- and 4"'- $\left.\mathrm{H}_{2}\right), 2.83\left(\mathrm{~m}, 2 \mathrm{H}, 6-\mathrm{H}_{2}\right)$, 3.19 (m, 1H, 1"-H), 3.56 (d, 1H, J = 7.0 Hz, 17-H), 3.77 (s, 3H, 3-OCH $), 4.43$ (m, 2H, 16a-H2), $6.62(\mathrm{~s}, 1 \mathrm{H}, 4-\mathrm{H}), 6.70(\mathrm{~d}, 1 \mathrm{H}, J=8.5 \mathrm{~Hz}, 2-\mathrm{H}), 7.19$ (d, 1H, $J=8.5 \mathrm{~Hz}, 1-\mathrm{H}), 7.35$ (s, 1H, $\left.5^{\prime}-\mathrm{H}\right)$. ${ }^{13} \mathrm{C}$ NMR ( $\delta$, ppm, $\mathrm{CDCl}_{3}$ ): 11.9 (C-18), 25.1 (C-3" and -4"), 26.1, 27.2, 28.3, 29.7 (C-2" and 5”), 33.2, 36.6, 38.4, 43.9, 44.2, 44.3 (C-13), 48.4, $55.2\left(3-\mathrm{OCH}_{3}\right), 62.1$ (C-16a), $85.3(\mathrm{C}-17)$, 111.5 (C-2), 113.8 (C-4), 126.3 (C-1), 132.3 (C-10), 137.8 (C-5), 157.5 (C-3).

\subsubsection{3-Methoxy-16a-(4'-cyclohexyl-1 'H-1',2',3'-triazol-1'-yl)methylestra-1,3,5(10)-trien-17 $\beta$ -} ol $(22 c)$

Compound 14 (342 mg, $1 \mathrm{mmol})$ and cyclohexylacetylene ( $2 \mathrm{mmol}, 0.22 \mathrm{ml})$ were used for the synthesis as described in Section 2.3. The crude product was chromatographed on silica gel with ethyl acetate $/ \mathrm{CH}_{2} \mathrm{Cl}_{2}(5: 95 \mathrm{v} / \mathrm{v})$ to yield pure $22 \mathrm{c}(345 \mathrm{mg}, 76 \%)$ as a white solid. Mp: 80-82 ${ }^{\circ} \mathrm{C}$; $R_{\mathrm{f}}=0.34$ (ss B). (Found 74.96; H, 8.54. $\mathrm{C}_{28} \mathrm{H}_{41} \mathrm{~N}_{3} \mathrm{O}_{2}$ (449.63) requires $\mathrm{C}, 74.80 ; \mathrm{H}, 8.74 \%$ ). ${ }^{1} \mathrm{H}$ $\operatorname{NMR}\left(\delta, \mathrm{ppm}, \mathrm{CDCl}_{3}\right): 0.83\left(\mathrm{~s}, 3 \mathrm{H}, 18-\mathrm{H}_{3}\right), 2.83\left(\mathrm{~m}, 2 \mathrm{H}, 6-\mathrm{H}_{2}\right), 3.55$ (s, 1H, 17-H), 3.77 (s, 3H, $\left.3-\mathrm{OCH}_{3}\right), 4.46\left(\mathrm{~s}, 2 \mathrm{H}, 16 \mathrm{a}-\mathrm{H}_{2}\right), 6.62(\mathrm{~d}, 1 \mathrm{H}, J=2.0 \mathrm{~Hz}, 4-\mathrm{H}), 6.70(\mathrm{dd}, 1 \mathrm{H}, J=8.5 \mathrm{~Hz}, J=2.0$ $\mathrm{Hz}, 2-\mathrm{H}), 7.19(\mathrm{~d}, 1 \mathrm{H}, J=8.5 \mathrm{~Hz}, 1-\mathrm{H}) .{ }^{13} \mathrm{C} \mathrm{NMR}\left(\delta, \mathrm{ppm}, \mathrm{CDCl}_{3}\right): 11.9(\mathrm{C}-18), 26.0$ and 26.1

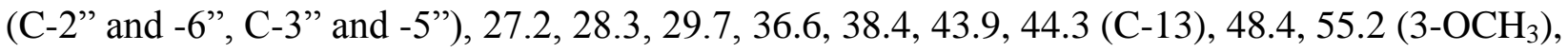
62.1 (C-1”), 62.1 (C-16a), 85.2 (C-17), 111.5 (C-2), 113.8 (C-4), 126.2 (C-1), 132.3 (C-10), 137.8 (C-5), $157.4(\mathrm{C}-3)$.

\subsubsection{3-Methoxy-16a-(4'-phenyl-1'H-1',2',3'-triazol-1'-yl)methylestra-1,3,5(10)-trien-17 $\beta$-ol $(22 d)$}

Compound $14(342 \mathrm{mg}, 1 \mathrm{mmol})$ and phenylacetylene $(2 \mathrm{mmol}, 0.22 \mathrm{ml})$ were used for the synthesis as described in Section 2.3. The crude product was chromatographed on silica gel ethyl acetate $/ \mathrm{CH}_{2} \mathrm{Cl}_{2}(5: 95 \mathrm{v} / \mathrm{v})$ to yield pure $22 \mathrm{~d}(368 \mathrm{mg}, 82 \%)$ as a white solid. $\mathrm{Mp}$ : $204-205{ }^{\circ} \mathrm{C}$; $R_{\mathrm{f}}=0.38$ (ss B). (Found C, 75.63; H, 7.72. $\mathrm{C}_{28} \mathrm{H}_{33} \mathrm{~N}_{3} \mathrm{O}_{2}$ (443.58) requires C, 75.81; H, 7.50\%). ${ }^{1} \mathrm{H}$ NMR ( $\delta$, ppm, DMSO-d $\left.)_{6}\right): 0.73\left(\mathrm{~s}, 3 \mathrm{H}, 18-\mathrm{H}_{3}\right), 2.73\left(\mathrm{~m}, 2 \mathrm{H}, 6-\mathrm{H}_{2}\right), 3.67$ (s, 3H, 3-OCH $), 4.36(\mathrm{t}$, 
$\left.1 \mathrm{H}, J=13.5 \mathrm{~Hz}, 16 \mathrm{a}-\mathrm{H}_{2}\right), 4.54\left(\mathrm{dd}, 1 \mathrm{H}, J=13.5 \mathrm{~Hz}, J=4.0 \mathrm{~Hz}, 16 \mathrm{a}-\mathrm{H}_{2}\right), 4.91(\mathrm{~d}, 1 \mathrm{H}, J=4.0 \mathrm{~Hz}$, 17-H), 6.58 (s, 1H, 4-H), 6.67 (d, 1H, $J=8.5 \mathrm{~Hz}, 2-\mathrm{H}), 7.15$ (d, 1H, $J=8.5 \mathrm{~Hz}, 1-\mathrm{H}), 7.32$ (t, 1H, $J=7.0 \mathrm{~Hz}, 4$ "'-H), 7.44 (t, 2H, $J=7.0 \mathrm{~Hz}, 3$ "'- and 5"-H), 7.86 (d, 2H, $J=7.0 \mathrm{~Hz}, 2$ "- and 6"-H), $8.61\left(\mathrm{~s}, 1 \mathrm{H}, 5\right.$ '-H) ${ }^{13} \mathrm{C}$ NMR ( $\delta$, ppm, DMSO-d $\left.{ }_{6}\right): 11.8$ (C-18), 25.8, 26.7, 27.3, 29.1, 36.3, 38.1, 43.4, 43.5, 43.8, 47.5, 53.5 (C-13), $54.8\left(3-\mathrm{OCH}_{3}\right), 83.1$ (C-17), 111.4 (C-2), 113.3 (C-4), 121.4 (C-5'), 125.0 (C-2" and -6”), 126.0 (C-1), 127.6 (C-4"), 128.8 (C-3" and -5”), 130.8 (C-1"), 132.0 (C-10), 137.3 (C-5), 146.1 (C-4'), 156.9 (C-3).

\subsubsection{3-Methoxy-16a-[4'(4' '-nitro-benzoyloxymethyl)-1 'H-1',2',3'-triazol-1'-yl]methylestra- 1,3,5(10)-trien-17 $\beta$-ol (22e)}

Compound 14 (342 mg, $1 \mathrm{mmol}$ ) and propargyl 4-nitrobenzoate ( $2 \mathrm{mmol}, 410 \mathrm{mg}$ ) were used for the synthesis as described in Section 2.3. The crude product was chromatographed on silica gel with ethyl acetate/ $\mathrm{CH}_{2} \mathrm{Cl}_{2}(5: 95 \mathrm{v} / \mathrm{v})$ to yield pure $22 \mathrm{e}(445 \mathrm{mg}, 81 \%)$ as a yellow solid. Mp: $86-88{ }^{\circ} \mathrm{C} ; R_{\mathrm{f}}=0.28$ (ss B). (Found C, 66.08; H, 6.43. $\mathrm{C}_{30} \mathrm{H}_{34} \mathrm{~N}_{4} \mathrm{O}_{6}(546.61$ ) requires $\mathrm{C}, 65.92 ; \mathrm{H}$, 6.27\%). ${ }^{1} \mathrm{H}$ NMR ( $\delta$, ppm, DMSO-d 6 ): 0.69 (s, 3H, 18- $\mathrm{H}_{3}$ ), 2.68 (m, 2H, 6- $\mathrm{H}_{2}$ ), 3.57 (s, 3H, 3$\left.\mathrm{OCH}_{3}\right), 4.38\left(\mathrm{dd}, 1 \mathrm{H}, J=13.5 \mathrm{~Hz}, J=9.0 \mathrm{~Hz}, 16 \mathrm{a}-\mathrm{H}_{2}\right), 4.52(\mathrm{dd}, 1 \mathrm{H}, J=13.5 \mathrm{~Hz}, J=4.5 \mathrm{~Hz}$, $\left.16 \mathrm{a}-\mathrm{H}_{2}\right), 4.86(\mathrm{~d}, 1 \mathrm{H}, J=4.5 \mathrm{~Hz}, 17-\mathrm{H}), 5.46\left(\mathrm{~s}, 2 \mathrm{H}, 4^{\prime}-\mathrm{H}_{2}\right), 6.55(\mathrm{~d}, 1 \mathrm{H}, J=1.5 \mathrm{~Hz}, 4-\mathrm{H}), 6.63$ (dd, $1 \mathrm{H}, J=8.5 \mathrm{~Hz}, 2-\mathrm{H}), 7.10(\mathrm{~d}, 1 \mathrm{H}, J=8.5 \mathrm{~Hz}, 1-\mathrm{H}), 8.16(\mathrm{~d}, 2 \mathrm{H}, J=8.5 \mathrm{~Hz}, 3$ "- and 5"-H), $8.28\left(\mathrm{~d}, 2 \mathrm{H}, J=8.5 \mathrm{~Hz}, 2\right.$ "- and 6"-H), 8.31 (s, $\left.1 \mathrm{H}, 5^{\prime}-\mathrm{H}\right) .{ }^{13} \mathrm{C}$ NMR ( $\delta$, ppm, DMSO-d 6 ): 11.7 (C-18), 25.7, 26.6, 27.1, 29.0, 36.4, 38.0, 43.3, 43.4 (C-13), 43.7, 47.7, 53.1 (C-16a), 54.7 (3$\mathrm{OCH}_{3}$ ), $58.6\left(\right.$ (") $\left.^{-\mathrm{CH}_{2}}\right), 82.8$ (C-17), $111.3(\mathrm{C}-2), 113.3$ (C-4), 123.8 (C-2" and -6"), 125.2 (C-5'), 125.9 (C-1), 130.6 (C-3" and -5”), 131.8 (C-10), 134.7 (C-1'), 137.2 (C-5), 141.1 (C-4"), 150.2 (C-4'), $156.9(\mathrm{C}-3), 163.9(\mathrm{C}=\mathrm{O})$.

2.3.12. 3-Methoxy-16a-(4'-hydroxymethyl-1'H-1',2'3'-triazol-1'-yl)methylestra-1,3,5(10)-trien$17 \beta-o l(22 f)$

Compound $22 \mathrm{e}$ ( $274 \mathrm{mg}, 0.5 \mathrm{mmol}$ ) was dissolved in methanol $(10 \mathrm{ml})$ containing $\mathrm{NaOCH}_{3}$ (14 $\mathrm{mg}, 0.25 \mathrm{mmol}$ ), and the solution was allowed to stand for $24 \mathrm{~h}$. It was then diluted with water, and the precipitate separating out was filtered off and recrystallized from a mixture of ethyl acetate/hexane to afford $22 \mathrm{f}(175 \mathrm{mg}, 88 \%)$ as a white crystalline product. Mp: $98-100{ }^{\circ} \mathrm{C} ; R_{\mathrm{f}}=$ 
0.28 (ss B). (Found C, 69.74; H, 7.72. $\mathrm{C}_{23} \mathrm{H}_{31} \mathrm{~N}_{3} \mathrm{O}_{3}$ (397.51) requires $\mathrm{C}, 69.49 ; \mathrm{H}, 7.86 \%$ ). ${ }^{1} \mathrm{H}$ $\operatorname{NMR}\left(\delta, \mathrm{ppm}, \mathrm{CDCl}_{3}\right): 0.81\left(\mathrm{~s}, 3 \mathrm{H}, 18-\mathrm{H}_{3}\right), 2.82\left(\mathrm{~m}, 2 \mathrm{H}, 6-\mathrm{H}_{2}\right), 3.50(\mathrm{~d}, 1 \mathrm{H}, J=7.0 \mathrm{~Hz}, 17-\mathrm{H})$, $3.76\left(\mathrm{~s}, 3 \mathrm{H}, 3-\mathrm{OCH}_{3}\right), 4.42\left(\mathrm{~d}, 2 \mathrm{H}, J=7.0 \mathrm{~Hz}, 16 \mathrm{a}-\mathrm{H}_{2}\right), 4.71\left(\mathrm{~s}, 2 \mathrm{H}, 4^{\prime}-\mathrm{H}_{2}\right), 6.61(\mathrm{~s}, 1 \mathrm{H}, 4-\mathrm{H})$, $6.69(\mathrm{~d}, 1 \mathrm{H}, J=8.5 \mathrm{~Hz}, 2-\mathrm{H}), 7.17(\mathrm{~d}, 1 \mathrm{H}, J=8.5 \mathrm{~Hz}, 1-\mathrm{H}), 7.68\left(\mathrm{~s}, 1 \mathrm{H}, 5^{\prime}-\mathrm{H}\right) .{ }^{13} \mathrm{C}$ NMR $(\delta$, ppm, $\mathrm{CDCl}_{3}$ ): 11.9 (C-18), 26.1, 27.2, 28.2, 29.6, 36.5, 38.4, 43.8, 44.0, 44.4 (C-13), 48.2, 54.6 (C-16a), $55.2\left(3-\mathrm{OCH}_{3}\right), 56.0$ (4'- $\left.\mathrm{CH}_{2}\right), 85.1$ (C-17), 111.5 (C-2), 113.8 (C-4), 126.3 (C-1), 132.3 (C-10), 137.8 (C-5), 157.4 (C-3).

2.3.13. 3-Methoxy-16a-(4'-cyclopropyl-1'H-1',2',3'-triazol-1'-yl)methylestra-1,3,5(10)-trien$17 \beta-o l(23 a)$

Compound 15 (342 mg, $1 \mathrm{mmol}$ ) and cyclopropylacetylene ( $2 \mathrm{mmol}, 0.22 \mathrm{ml})$ were used for the synthesis as described in Section 2.3. The crude product was chromatographed on silica gel with ethyl acetate $/ \mathrm{CH}_{2} \mathrm{Cl}_{2}(1: 99 \mathrm{v} / \mathrm{v})$ to yield pure $23 \mathbf{a}(261 \mathrm{mg}, 64 \%)$ as a white solid. Mp: $67-69{ }^{\circ} \mathrm{C}$; $R_{\mathrm{f}}=0.32$ (ss B). (Found C, 73.85; H, 8.32. $\mathrm{C}_{25} \mathrm{H}_{33} \mathrm{~N}_{3} \mathrm{O}_{2}$ (407.55) requires $\mathrm{C}, 73.68 ; \mathrm{H}, 8.16 \%$ ). ${ }^{1} \mathrm{H}$ $\operatorname{NMR}\left(\delta, \mathrm{ppm}, \mathrm{CDCl}_{3}\right): 0.82\left(\mathrm{~m}, 5 \mathrm{H}, 18-\mathrm{H}_{3}\right.$ and cyclopropyl- $\left.\mathrm{H}_{2}\right), 0.95\left(\mathrm{~m}, 2 \mathrm{H}\right.$, cyclopropyl- $\left.\mathrm{H}_{2}\right)$, $2.83\left(\mathrm{~m}, 2 \mathrm{H}, 6-\mathrm{H}_{2}\right), 3.53(\mathrm{~d}, 1 \mathrm{H}, J=7.5 \mathrm{~Hz}, 17-\mathrm{H}), 3.77$ (s, 3H, 3- $\left.\mathrm{OCH}_{3}\right), 4.35$ (t, $1 \mathrm{H}, J=7.5 \mathrm{~Hz}$, $\left.16 \mathrm{a}-\mathrm{H}_{2}\right), 4.44\left(\mathrm{dd}, 1 \mathrm{H}, J=13.5 \mathrm{~Hz}, J=7.5 \mathrm{~Hz}, 16 \mathrm{a}-\mathrm{H}_{2}\right), 6.62(\mathrm{~d}, 1 \mathrm{H}, J=2.0 \mathrm{~Hz}, 4-\mathrm{H}), 6.70(\mathrm{dd}$, $1 \mathrm{H}, J=8.5 \mathrm{~Hz}, J=2.0 \mathrm{~Hz}, 2-\mathrm{H}), 7.18(\mathrm{~d}, 1 \mathrm{H}, J=8.5 \mathrm{~Hz}, 1-\mathrm{H}) .{ }^{13} \mathrm{C} \mathrm{NMR}\left(\delta, \mathrm{ppm}, \mathrm{CDCl}_{3}\right): 6.7$ (C-1"), 7.7 (C-2" and -3"), 11.8 (C-18), 26.1, 27.2, 28.2, 29.7, 36.6, 38.4, 43.9, 44.3, 44,3 (C16a), 48.3, 54.5 (C-13), 62.1 (3- $\left.\mathrm{OCH}_{3}\right), 85.1$ (C-17), 111.5 (C-2), 113.8 (C-4), 126.2 (C-1), 132.3 (C-10), 137.8 (C-5), 157.4 (C-3).

2.3.14. 3-Methoxy-16ß-(4'-cyclopentyl-1'H-1',2',3'-triazol-1-yl)methylestra-1,3,5(10)-trien-17aol $(23 b)$

Compound 15 (342 mg, $1 \mathrm{mmol}$ ) and cyclopentylacetylene ( $2 \mathrm{mmol}, 0.22 \mathrm{ml})$ were used for the synthesis as described in Section 2.3. The crude product was chromatographed on silica gel with ethyl acetate $/ \mathrm{CH}_{2} \mathrm{Cl}_{2}(1: 99 \mathrm{v} / \mathrm{v})$ to yield pure $\mathbf{2 3 b}(380 \mathrm{mg}, 87 \%)$ as yellow crystalline material. Mp: 67-68 ${ }^{\circ} \mathrm{C} ; R_{\mathrm{f}}=0.36$ (ss B). (Found C, 74.28; H, 8.47. $\mathrm{C}_{27} \mathrm{H}_{37} \mathrm{~N}_{3} \mathrm{O}_{2}$ (435.60) requires $\mathrm{C}$, 74.45; H, 8.56\%). ${ }^{1} \mathrm{H}$ NMR ( $\left.\delta, \mathrm{ppm}, \mathrm{CDCl}_{3}\right): 0.75\left(\mathrm{~s}, 3 \mathrm{H}, 18-\mathrm{H}_{3}\right), 2.85\left(\mathrm{~m}, 2 \mathrm{H}, 6-\mathrm{H}_{2}\right), 3.68(\mathrm{~s}$, 1H, 17-H), 3.77 (s, 3H, 3-OCH $), 4.44\left(\mathrm{~d}, 2 \mathrm{H}, J=15.0 \mathrm{~Hz}, 16 \mathrm{a}-\mathrm{H}_{2}\right), 6.62(\mathrm{~s}, 1 \mathrm{H}, 4-\mathrm{H}), 6.70$ (d, $1 \mathrm{H}, J=8.5 \mathrm{~Hz}, 2-\mathrm{H}), 7.20(\mathrm{t}, 1 \mathrm{H}, J=8.5 \mathrm{~Hz}, 1-\mathrm{H}) .{ }^{13} \mathrm{C} \mathrm{NMR}\left(\delta, \mathrm{ppm}, \mathrm{CDCl}_{3}\right): 17.9(\mathrm{C}-18), 25.1$ 
(C-3" and -4"), 25.9, 26.1, 27.2, 28.0, 29.7, 30.4, 31.8, 36.6 (C-16a), 38.5, 43.3, 43.8, 45.1 (C13), 48.9, 55.2 (3- $\left.\mathrm{OCH}_{3}\right), 62.1$ (C-1”), 82.6 (C-17), 111.5 (C-2), 113.7 (C-4), 113.8 (C-5'), 126.2 (C-1), 132.1 (C-10), 137.8 (C-5), 137.8 (C-4'), 157.4 (C-3).

\subsubsection{3-Methoxy-168-(4'-cyclohexyl-1 'H-1',2',3'-triazol-1'-yl)methyestra-1,3,5(10)-trien-17a- ol (23c)}

Compound $15(342,1 \mathrm{mmol})$ and cyclohexylacetylene $(2 \mathrm{mmol}, 0.22 \mathrm{ml})$ were used for the synthesis as described in Section 2.3. The crude product was chromatographed on silica gel with ethyl acetate $/ \mathrm{CH}_{2} \mathrm{Cl}_{2}(5: 95 \mathrm{v} / \mathrm{v})$ to yield pure $23 \mathrm{c}(306 \mathrm{mg}, 68 \%)$ as a white solid. Mp: $90-92{ }^{\circ} \mathrm{C}$; $R_{\mathrm{f}}=0.37$ (ss B). (Found C, 74.95; H, 8.83. $\mathrm{C}_{28} \mathrm{H}_{41} \mathrm{~N}_{3} \mathrm{O}_{2}$ (449.63) requires $\mathrm{C}, 74.80 ; \mathrm{H}, 8.74 \%$ ). ${ }^{1} \mathrm{H}$ $\operatorname{NMR}\left(\delta, \mathrm{ppm}, \mathrm{CDCl}_{3}\right): 0.75\left(\mathrm{~s}, 3 \mathrm{H}, 18-\mathrm{H}_{3}\right), 2.84\left(\mathrm{~m}, 2 \mathrm{H}, 6-\mathrm{H}_{2}\right), 3.67(\mathrm{~d}, 1 \mathrm{H}, J=1.0 \mathrm{~Hz}, 17-\mathrm{H})$, $3.77\left(\mathrm{~S}, 3 \mathrm{H}, 3-\mathrm{OCH}_{3}\right), 4.43\left(\mathrm{~m}, 1 \mathrm{H}, 16 \mathrm{a}-\mathrm{H}_{2}\right), 6.62(\mathrm{~d}, 1 \mathrm{H}, J=2.5 \mathrm{~Hz}, 4-\mathrm{H}), 6.71(\mathrm{dd}, 1 \mathrm{H}, J=8.5$ $\mathrm{Hz}, J=2.5 \mathrm{~Hz}, 2-\mathrm{H}), 7.20(\mathrm{t}, 1 \mathrm{H}, J=8.5 \mathrm{~Hz}, 1-\mathrm{H}), 7.35\left(\mathrm{~s}, 1 \mathrm{H}, 5^{\prime}-\mathrm{H}\right) .{ }^{13} \mathrm{C}$ NMR $(\delta, \mathrm{ppm}$, $\mathrm{CDCl}_{3}$ ): 17.9 (C-18), 25.9, 26.0, 26.1 (C-2" and -6"), 28.0, 29.7, 30.4, 31.8, 33.0, 35.2 (C-1"), 36.6, 38.5, 43.3, 45.1 (C-13), 48.9, 49.1, 54.3 (C-16a), $55.2\left(3-\mathrm{OCH}_{3}\right), 82.6(\mathrm{C}-1), 132.4(\mathrm{C}-10)$, 137.8 (C-5), 153.7 (C-4'), 157.7 (C-3).

2.3.16. 3-Methoxy-16ß-(4'-phenyl-1'H-1',2',3'-triazol-1'-yl)methy-estra-1,3,5(10)-trien-17a-ol (23d)

Compound $15(342 \mathrm{mg}, 1 \mathrm{mmol})$ and phenylacetylene $(2 \mathrm{mmol}, 0.22 \mathrm{ml})$ were used for the synthesis as described in Section 2.3. The crude product was chromatographed on silica gel with ethyl acetate $/ \mathrm{CH}_{2} \mathrm{Cl}_{2}(2.5: 97.5 \mathrm{v} / \mathrm{v})$ to yield pure $23 \mathrm{~d}(299 \mathrm{mg}, 67 \%)$ as white crystals. Mp: $173-174{ }^{\circ} \mathrm{C} ; R_{\mathrm{f}}=0.34$ (ss B). (Found C 75.98; H, 7.33. $\mathrm{C}_{28} \mathrm{H}_{33} \mathrm{~N}_{3} \mathrm{O}_{2}$ (443.58) requires C, 75.81; $\mathrm{H}, 7.50 \%) .{ }^{1} \mathrm{H}$ NMR $\left(\delta, \mathrm{ppm}, \mathrm{CDCl}_{3}\right): 0.79$ (s, 3H, 18- $\left.\mathrm{H}_{3}\right), 2.85\left(\mathrm{~m}, 2 \mathrm{H}, 6-\mathrm{H}_{2}\right), 3.71(\mathrm{~d}, 1 \mathrm{H}, J=$ $1.5 \mathrm{~Hz}, 17-\mathrm{H}), 3.78\left(\mathrm{~s}, 3 \mathrm{H}, 3-\mathrm{OCH}_{3}\right), 4.46\left(\mathrm{dd}, 1 \mathrm{H}, J=13.5 \mathrm{~Hz}, J=8.0 \mathrm{~Hz}, 16 \mathrm{a}-\mathrm{H}_{2}\right), 4.55$ (dd, $\left.1 \mathrm{H}, J=13.5 \mathrm{~Hz}, J=8.0 \mathrm{~Hz}, 16 \mathrm{a}-\mathrm{H}_{2}\right), 6.63(\mathrm{~d}, 1 \mathrm{H}, J=2.0 \mathrm{~Hz}, 4-\mathrm{H}), 6.72(\mathrm{dd}, 1 \mathrm{H}, J=8.5 \mathrm{~Hz}, J=$ $2.5 \mathrm{~Hz}, 2-\mathrm{H}), 7.21$ (d, 1H, $J=8.5 \mathrm{~Hz}, 1-\mathrm{H}), 7.27$ (t, $1 \mathrm{H} J=7.5 \mathrm{~Hz}, 4$ "-H), 7.42 (t, 2H, $J=7.5 \mathrm{~Hz}$, 3"- and 5"-H), $7.83\left(\mathrm{~d}, 2 \mathrm{H}, J=7.5 \mathrm{~Hz}, 2\right.$ "- and 6"-H), $7.87\left(\mathrm{~s}, 1 \mathrm{H}, 5\right.$ '-H). ${ }^{13} \mathrm{C}$ NMR $(\delta, \mathrm{ppm}$, $\mathrm{CDCl}_{3}$ ): 17.9 (C-18), 25.9, 27.9, 29.7, 30.4, 31.8, 38.5, 43.3, 45.1, (C-13), 48.8, 49.1, 54.5 (C16a), $55.2\left(3-\mathrm{OCH}_{3}\right), 82.5$ (C-17), $111.5(\mathrm{C}-2), 113.7$ (C-4), 119.6 (C-5'), 125.7 (C-2" and -6"), 
126.3 (C-1), 128.1 (C-4"), 128.8 (C-3" and -5"), 130.5 (C-1"), 132.4 (C-10), 137.8 (C-5), 147.8 (C-4'), 157.4 (C-3).

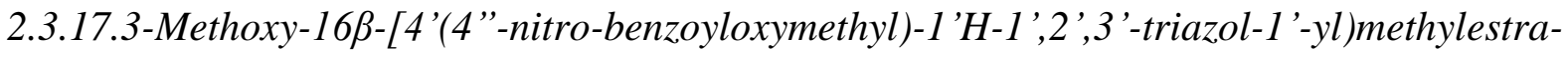 1,3,5(10)-trien-17a-ol (23e)}

Compound 15 (342, $1 \mathrm{mmol})$ and propargyl 4-nitro benzoate ( $2 \mathrm{mmol}, 410 \mathrm{mg}$ ) were used for the synthesis as described in Section 2.3. The crude product was chromatographed on silica gel with ethyl acetate $/ \mathrm{CH}_{2} \mathrm{Cl}_{2}(5: 95 \mathrm{v} / \mathrm{v})$ to yield pure $23 \mathrm{e}(370 \mathrm{mg}, 67 \%)$ as a yellow crystalline material. Mp: 62-63 ${ }^{\circ} \mathrm{C} ; R_{\mathrm{f}}=0.38$ (ss B). (Found C, 66.14; H, 6.42. $\mathrm{C}_{30} \mathrm{H}_{34} \mathrm{~N}_{4} \mathrm{O}_{6}$ (546.61) requires $\mathrm{C}$, 65.92; H, 6.27\%). ${ }^{1} \mathrm{H}$ NMR ( $\delta$, ppm, DMSO-d $\left.{ }_{6}\right): 0.65\left(\mathrm{~s}, 3 \mathrm{H}, 18-\mathrm{H}_{3}\right), 2.74\left(\mathrm{~m}, 2 \mathrm{H}, 6-\mathrm{H}_{2}\right), 3.68(\mathrm{~s}$, $\left.3 \mathrm{H}, 3-\mathrm{OCH}_{3}\right), 4.41\left(\mathrm{dd}, 1 \mathrm{H}, J=13.0 \mathrm{~Hz}, J=8.5 \mathrm{~Hz}, 16 \mathrm{a}-\mathrm{H}_{2}\right), 4.56(\mathrm{dd}, 1 \mathrm{H}, J=13.0 \mathrm{~Hz}, J=8.5$ Hz, 16a- $\left.\mathrm{H}_{2}\right), 4.63$ (d, 1H, J = 4.5 Hz, 17-H), 6.58 (s, 1H, 4-H), 6.66 (d, 1H, J = 8.5 Hz, 2-H), 7.16 (d, $1 \mathrm{H}, J=8.5 \mathrm{~Hz}, 1-\mathrm{H}), 8.19$ (d, 2H, $J=8.5 \mathrm{~Hz}, 3$ "- and 5"-H), 8.34 (d, $2 \mathrm{H}, J=8.5 \mathrm{~Hz}, 2$ "- and 6 "-H). ${ }^{13} \mathrm{C}$ NMR ( $\delta$, ppm, DMSO-d 6 ): 17.5 (C-18), 25.6, 27.5, 29.6, 31.8, 38.2, 43.0, 44.5, 47.9 (C-13), 48.2, 49.1, 53.6 (C-16a), 54.8 (3- $\left.\mathrm{OCH}_{3}\right), 58.7$ (4'- $\left.\mathrm{CH}_{2}\right), 80.8$ (C-17), 111.3 (C-2), 113.3 (C-4), 123.8 (C-1), 126.1 (C-5'), 130.6 (C-2" and -6"), 131.9 (C-3" and -5"), 133.0 (C-10), 134.7 (C-1"), 137.3 (C-5), 141.4 (C-4”), 150.2 (C-4'), 156.9 (C-3), 163.9 (C=O).

2.3.18. 3-Methoxy-163-(4'-hydroxymethyl-1 'H-1',2'3'-triazol-1'-yl)methylestra-1,3,5(10)-trien$17 a-o l(23 f)$

Compound 23e (274 mg, $0.5 \mathrm{mmol})$ was dissolved in methanol $(10 \mathrm{ml})$ containing $\mathrm{NaOCH}_{3}(14$ $\mathrm{mg}, 0.25 \mathrm{mmol}$ ), and the solution was allowed to stand for $24 \mathrm{~h}$. It was then diluted with water, and the precipitate separating out was filtered off, dissolved in dichloromethane and washed with water. The organic phase was dried over $\mathrm{Na}_{2} \mathrm{SO}_{4}$, and evaporated in vacuo to afford $23 \mathbf{f f}$ (183 $\mathrm{mg}$, $92 \%$ ) as oil. $R_{\mathrm{f}}=0.26$ (ss B). (Found $\mathrm{C}, 69.28 ; \mathrm{H}, 7.95 . \mathrm{C}_{23} \mathrm{H}_{31} \mathrm{~N}_{3} \mathrm{O}_{3}$ (397.51) requires C, 69.49; H, 7.86\%). ${ }^{1} \mathrm{H}$ NMR ( $\left.\delta, \mathrm{ppm}, \mathrm{CDCl}_{3}\right): 0.78$ (s, 3H, 18- $\left.\mathrm{H}_{3}\right), 2.85$ (m, 2H, 6- $\left.\mathrm{H}_{2}\right), 3.65$ (s, 1H, $17-$ $\mathrm{H}), 3.77\left(\mathrm{~s}, 3 \mathrm{H}, 3-\mathrm{OCH}_{3}\right), 4.46\left(\mathrm{~m}, 2 \mathrm{H}, 16 \mathrm{a}-\mathrm{H}_{2}\right), 4.78\left(\mathrm{~s}, 2 \mathrm{H}, 4^{\prime}-\mathrm{H}_{2}\right), 6.62(\mathrm{~d}, 1 \mathrm{H}, J=2.0 \mathrm{~Hz}, 4-$ $\mathrm{H}), 6.72(\mathrm{dd}, 1 \mathrm{H}, J=8.5 \mathrm{~Hz}, J=2.5 \mathrm{~Hz}, 2-\mathrm{H}), 7.19(\mathrm{~d}, 1 \mathrm{H}, J=8.5 \mathrm{~Hz}, 1-\mathrm{H}) .{ }^{13} \mathrm{C} \mathrm{NMR}(\delta, \mathrm{ppm}$, $\mathrm{CDCl}_{3}$ ): 17.9 (C-18), 25.9, 27.9, 29.7, 30.3, 31.8, 38.5, 43.3, 45.2 (C-13), 48.8, 49.2, 54.6 (C16a), $55.2\left(3-\mathrm{OCH}_{3}\right), 56.1$ (4'- $\left.\mathrm{CH}_{2}\right), 82.1$ (C-17), 111.5 (C-2), 113.7 (C-4), 123.5 (C-5'), 126.3 (C-1), 132.4 (C-10), 137.8 (C-5), 157.4 (C-3). 
2.3.19. 3-Methoxy-16a-(4'-cyclopropyl-1 'H-1',2',3'-triazol-1'-yl)methylestra-1,3,5(10)-trien$17 a-o l(24 a)$

Compound 16 (342 mg, $1 \mathrm{mmol})$ and cyclopropylacetylene $(2 \mathrm{mmol}, 0.22 \mathrm{ml})$ were used for the synthesis as described in Section 2.3. The crude product was chromatographed on silica gel with ethyl acetate $/ \mathrm{CH}_{2} \mathrm{Cl}_{2}(2.5: 97.5 \mathrm{v} / \mathrm{v})$ to yield pure $24 \mathrm{a}(310 \mathrm{mg}, 76 \%)$ as a white solid. $\mathrm{Mp}$ : $165-166{ }^{\circ} \mathrm{C} ; R_{\mathrm{f}}=0.40$ (ss B). (Found $\mathrm{C}, 73.85 ; \mathrm{H}, 8.34 . \mathrm{C}_{25} \mathrm{H}_{33} \mathrm{~N}_{3} \mathrm{O}_{2}$ (407.55) requires $\mathrm{C}$, 73.68; $\mathrm{H}, 8.16 \%) .{ }^{1} \mathrm{H}$ NMR $\left(\delta, \mathrm{ppm}, \mathrm{CDCl}_{3}\right): 0.74$ (s, 3H, 18- $\left.\mathrm{H}_{3}\right), 0.85$ and $0.96(2 \mathrm{x} \mathrm{m}, 4 \mathrm{H}, 2$ "- and 3"$\left.\mathrm{H}_{2}\right), 2.85\left(\mathrm{~m}, 2 \mathrm{H}, 6-\mathrm{H}_{2}\right), 3.63(\mathrm{~d}, 1 \mathrm{H}, J=5.0 \mathrm{~Hz}, 17-\mathrm{H}), 3.77\left(\mathrm{~s}, 3 \mathrm{H}, 3-\mathrm{OCH}_{3}\right), 4.28(\mathrm{dd}, 1 \mathrm{H}, J=$ $\left.13.0 \mathrm{~Hz}, J=5.0 \mathrm{~Hz}, 16 \mathrm{a}-\mathrm{H}_{2}\right), 4.59\left(\mathrm{t}, 1 \mathrm{H}, J=12.0 \mathrm{~Hz}, 16 \mathrm{a}-\mathrm{H}_{2}\right), 6.63(\mathrm{~d}, 1 \mathrm{H}, J=2.0 \mathrm{~Hz}, 4-\mathrm{H})$, $6.71(\mathrm{dd}, 1 \mathrm{H}, J=8.5 \mathrm{~Hz}, J=2.5 \mathrm{~Hz}, 2-\mathrm{H}), 7.22(\mathrm{~d}, 1 \mathrm{H}, J=8.5 \mathrm{~Hz}, 1-\mathrm{H}) .{ }^{13} \mathrm{C}$ NMR $(\delta, \mathrm{ppm}$, $\mathrm{CDCl}_{3}$ ): 6.6 (C-1"), 7.7 and 7.8 (C-2" and -3"), 17.1 (C-18), 26.0, 28.0, 28.9, 29.8, 31.2, 38.9, 42.3, 46.3 (C-16a), 47.0, 50.5 (C-13), $55.2\left(3-\mathrm{OCH}_{3}\right), 78.8$ (C-17), 111.4 (C-2), 113.7 (C-4), 120.6 (C-5'), 126.3 (C-1), 132.5 (C-10), 137.9 (C-5), 149.8 (C-4'), 157.4 (C-3).

2.3.20. 3-Methoxy-16a-(4'-cyclopentyl-1 'H-1',2',3'-triazol-1'-yl)methyl-estra-1,3,5(10)-trien$17 a-o l(24 b)$

Compound 16 (342 mg, $1 \mathrm{mmol}$ ) and cyclopentylacetylene $(2 \mathrm{mmol}, 0.22 \mathrm{ml})$ were used for the synthesis as described in Section 2.3. The crude product was chromatographed on silica gel with ethyl acetate $/ \mathrm{CH}_{2} \mathrm{Cl}_{2}(1: 99 \mathrm{v} / \mathrm{v})$ to yield pure $\mathbf{2 4 b}(383 \mathrm{mg}, 88 \%)$ as yellow crystalline product. Mp: $171-173{ }^{\circ} \mathrm{C} ; R_{\mathrm{f}}=0.42$ (ss B). (Found C, 74.67; H, 8.72. $\mathrm{C}_{27} \mathrm{H}_{37} \mathrm{~N}_{3} \mathrm{O}_{2}$ (435.60) requires C, 74.45; H, 8.56\%). ${ }^{1} \mathrm{H}$ NMR ( $\delta$, ppm, $\left.\mathrm{CDCl}_{3}\right): 075$ (s, 3H, 18- $\left.\mathrm{H}_{3}\right), 1.25$ (s, 8H, 2"-, 3"-, 4"- and 5"- $\left.\mathrm{H}_{2}\right), 2.86\left(\mathrm{~m}, 2 \mathrm{H}, 6-\mathrm{H}_{2}\right), 3.18(\mathrm{~m}, 1 \mathrm{H}, 1$ "'-H), $3.64(\mathrm{~d}, 1 \mathrm{H}, J=5.0 \mathrm{~Hz}, 17-\mathrm{H}), 3.77$ (s, 3H, 3$\left.\mathrm{OCH}_{3}\right), 4.29\left(\mathrm{dd}, 1 \mathrm{H}, J=13.5 \mathrm{~Hz}, J=5.5 \mathrm{~Hz}, 16 \mathrm{a}-\mathrm{H}_{2}\right), 4.62(\mathrm{dd}, 1 \mathrm{H}, J=13.5 \mathrm{~Hz}, \mathrm{~J}=11.5 \mathrm{~Hz}$, $\left.16 \mathrm{a}-\mathrm{H}_{2}\right), 6.63(\mathrm{~d}, 1 \mathrm{H}, J=2.0 \mathrm{~Hz}, 4-\mathrm{H}), 6.71(\mathrm{dd}, 1 \mathrm{H}, J=8.5 \mathrm{~Hz}, J=2.0 \mathrm{~Hz}, 2-\mathrm{H}), 7.22(\mathrm{~d}, 1 \mathrm{H}, J$ $=8.5 \mathrm{~Hz}, 1-\mathrm{H}), 7.36$ (s, 1H, 5'-H). ${ }^{13} \mathrm{C}$ NMR ( $\delta$, ppm, $\mathrm{CDCl}_{3}$ ): 17.2 (C-18), 25.1 (C-3" and -4"), 26.0, 28.0, 29.0, 29.7, 29.9, 31.2, 33.2, 36.7, 38.9, 42.4, 43.5, 46.3 (C-13), 47.0 (C-1"), 50.5 (C16a), $55.2\left(3-\mathrm{OCH}_{3}\right), 78.8$ (C-17), 111.4 (C-2), 113.8 (C-4), 120.6 (C-5'), 126.3 (C-1), 132.6 (C10), 137.9 (C-5), 152.3 (C-4'), 157.4 (C-3). 
2.3.21. 3-Methoxy-16a-(4'-cyclohexyl-1 'H-1',2',3'-triazol-1'-yl)methylestra-1,3,5(10)-trien-17aol $(24 c)$

Compound 16 (342 mg, $1 \mathrm{mmol})$ and cyclohexylacetylene $(2 \mathrm{mmol}, 0.22 \mathrm{ml})$ were used for the synthesis as described in Section 2.3. The crude product was chromatographed on silica gel with ethyl acetate/ $\mathrm{CH}_{2} \mathrm{Cl}_{2}(1: 99 \mathrm{v} / \mathrm{v})$ to yield pure $\mathbf{2 4 c}$ (162 mg, 36\%) as yellow crystals. Mp: 208-210 ${ }^{\circ} \mathrm{C} ; R_{\mathrm{f}}=0.42$ (ss B). (Found C, 74.97; H, 8.56. $\mathrm{C}_{28} \mathrm{H}_{41} \mathrm{~N}_{3} \mathrm{O}_{2}$ (449.63) requires $\mathrm{C}, 74.80 ; \mathrm{H}, 8.74 \%$ ). ${ }^{1} \mathrm{H}$ NMR ( $\delta$, ppm, $\left.\mathrm{CDCl}_{3}\right)$ : 0.75 (s, 3H, 18- $\left.\mathrm{H}_{3}\right), 1.26$ (s, 8H, 2"-, 3"-, 5"- and 6"- $\left.\mathrm{H}_{2}\right), 2.88(\mathrm{~m}, 2 \mathrm{H}$, 6- $\mathrm{H}_{2}$ ), 2.90 (m, 2H, 4"- $\mathrm{H}_{2}$ ), 3.64 (d, 1H, $\left.J=5.0 \mathrm{~Hz}, 17-\mathrm{H}\right), 3.77$ (s, 3H, 3- $\mathrm{OCH}_{3}$ ), 4.29 (dd, 1H, $J$ $\left.=13.5 \mathrm{~Hz}, J=5.0 \mathrm{~Hz}, 16 \mathrm{a}-\mathrm{H}_{2}\right), 4.62\left(\mathrm{dd}, 1 \mathrm{H}, J=13.5 \mathrm{~Hz}, J=11.0 \mathrm{~Hz}, 16 \mathrm{a}-\mathrm{H}_{2}\right), 6.63(\mathrm{~d}, 1 \mathrm{H}, J=$ $2.0 \mathrm{~Hz}, 4-\mathrm{H}), 6.71(\mathrm{dd}, 1 \mathrm{H}, J=8.5 \mathrm{~Hz}, J=2.5 \mathrm{~Hz}, 2-\mathrm{H}), 7.22(\mathrm{~d}, 1 \mathrm{H}, J=8.5 \mathrm{~Hz}, 1-\mathrm{H}), 7.34$ (s, $\left.1 \mathrm{H}, 5^{\prime}-\mathrm{H}\right) .{ }^{13} \mathrm{C}$ NMR ( $\delta$, ppm, $\mathrm{CDCl}_{3}$ ): 17.2 (C-18), 26.0 and 26.1 (C-2”, -3", -5” and -6”), 28.0, 29.0, 29.7, 29.8, 31.2, 33.0, 25.2, 38.9, 42.4, 43.5, 46.3 (C-13), 47.0 (C-1”), 50.5 (C-16a), 55.0 (3-OCH ${ }_{3}$ ), 78.8 (C-17), 111.4 (C-2), 113.8 (C-4), 120.2 (C-5'), 126.3 (C-1), 132.6 (C-10), 137.9 (C-5), 153.3 (C-4'), 157.4 (C-3).

\subsubsection{3-Methoxy-16a-(4'-phenyl-1 'H-1',2',3'-triazol-1 '-yl)methylestra-1,3,5(10)-trien-17a-ol (24d)}

Compound $16342 \mathrm{mg}, 1 \mathrm{mmol})$ and phenylacetylene $(2 \mathrm{mmol}, 0.22 \mathrm{ml})$ were used for the synthesis as described in Section 2.3. The crude product was chromatographed on silica gel with $\mathrm{CH}_{2} \mathrm{Cl}_{2}$ yield pure $24 \mathrm{~d}$ (394 mg, 89\%) as white solid. Mp: $189.5-191{ }^{\circ} \mathrm{C} ; R_{\mathrm{f}}=0.46$ (ss B). (Found $\mathrm{C}, 75.65 ; \mathrm{H}, 7.67 . \mathrm{C}_{28} \mathrm{H}_{33} \mathrm{~N}_{3} \mathrm{O}_{2}$ (443.58) requires $\left.\mathrm{C}, 75.81 ; \mathrm{H}, 7.50 \%\right)$. ${ }^{1} \mathrm{H} \mathrm{NMR}\left(\delta, \mathrm{ppm}, \mathrm{CDCl}_{3}\right)$ : 0.75 (s, $\left.3 \mathrm{H}, 18-\mathrm{H}_{3}\right), 2.86\left(\mathrm{~m}, 2 \mathrm{H}, 6-\mathrm{H}_{2}\right), 3.68(\mathrm{~d}, 1 \mathrm{H}, J=5.0 \mathrm{~Hz}, 17-\mathrm{H}), 3.78\left(\mathrm{~s}, 3 \mathrm{H}, 3-\mathrm{OCH}_{3}\right)$, $4.41\left(\mathrm{dd}, 1 \mathrm{H}, J=13.5 \mathrm{~Hz}, J=6.0 \mathrm{~Hz}, 16 \mathrm{a}-\mathrm{H}_{2}\right), 4.69\left(\mathrm{dd}, 1 \mathrm{H}, J=14.5 \mathrm{~Hz}, J=10.5 \mathrm{~Hz}, 16 \mathrm{a}-\mathrm{H}_{2}\right)$, $6.64(\mathrm{~d}, 1 \mathrm{H}, J=2.0 \mathrm{~Hz}, 4-\mathrm{H}), 6.72(\mathrm{dd}, 1 \mathrm{H}, J=8.5 \mathrm{~Hz}, J=2.5 \mathrm{~Hz}, 2-\mathrm{H}), 7.22(\mathrm{~d}, 1 \mathrm{H}, J=8.5 \mathrm{~Hz}$, 1-H), 7.34 (t, 1H, $J=7.5 \mathrm{~Hz}, 4$ "-H), 7.43 (t, 2H, $J=7.5 \mathrm{~Hz}, 3$ "- and 5"-H), 7.83 (d, 2H, $J=7.5$ $\mathrm{Hz}, 2$ "- and 6"-H), 7.88 (s, 1H, 5'-H). ${ }^{13} \mathrm{C}$ NMR ( $\delta$, ppm, $\left.\mathrm{CDCl}_{3}\right): 17.1$ (C-18), 26.0, 28.0, 29.8, 31.2, 38.9, 42.3, 43.5, 46.4 (C-13), 47.0, 50.7, $55.2\left(3-\mathrm{OCH}_{3}\right), 78.8$ (C-17), $111.5(\mathrm{C}-2), 113.8$ (C-4), 120.6 (C-5'), 125.6 (C-2" and -6"), 126.3 (C-1), 128.1 (C-4"), 128.8 (C-3" and -5"), 130.5 (C-1”), 132.5 (C-10), 137.9 (C-5), 147.3 (C-4'), 157.4 (C-3). 


\subsubsection{3-Methoxy-16a-[4'-(4'"nitrobenzoyloxymethyl)-1'H-1',2',3'-triazol-1'-yl]methylestra-}

1,3,5(10)-trien-17a-ol (24e)

Compound 16 (342, $1 \mathrm{mmol})$ and propargyl 4-nitrobenzoate $(2 \mathrm{mmol}, 210 \mathrm{mg})$ were used for the synthesis as described in Section 2.3. The crude product was chromatographed on silica gel with $\mathrm{CH}_{2} \mathrm{Cl}_{2} /$ hexane $(1: 3, \mathrm{v} / \mathrm{v})$ to yield pure $(344 \mathrm{mg}, 63 \%)$ as yellow crystals. $\mathrm{Mp}: 64{ }^{\circ} \mathrm{C} ; R_{\mathrm{f}}=0.45$ (ss B). (Found, $\mathrm{C}, 66.14 ; \mathrm{H}, 6.05 . \mathrm{C}_{30} \mathrm{H}_{34} \mathrm{~N}_{4} \mathrm{O}_{6}(546.61)$ requires $\left.\mathrm{C}, 65.92 ; \mathrm{H}, 6.27 \%\right)$. ${ }^{1} \mathrm{H} \mathrm{NMR}(\delta$, ppm, $\left.\mathrm{CDCl}_{3}\right): 0.75$ (s, 3H, 18- $\left.\mathrm{H}_{3}\right), 2.84\left(\mathrm{~m}, 2 \mathrm{H}, 6-\mathrm{H}_{2}\right), 3.66(\mathrm{~d}, 1 \mathrm{H}, J=4.5 \mathrm{~Hz}, 17-\mathrm{H}), 3.77$ (s, $\left.3 \mathrm{H}, 3-\mathrm{OCH}_{3}\right), 4.40\left(\mathrm{dd}, 1 \mathrm{H}, J=13.5 \mathrm{~Hz}, J=5.5 \mathrm{~Hz}, 16 \mathrm{a}-\mathrm{H}_{2}\right), 4.66$ (t, $1 \mathrm{H}, J=13.5 \mathrm{~Hz}, 16 \mathrm{a}-\mathrm{H}_{2}$ ), $5.53\left(\mathrm{~s}, 2 \mathrm{H}, 4^{\prime}-\mathrm{H}_{2}\right), 6.62(\mathrm{t}, 1 \mathrm{H}, J=2.0 \mathrm{~Hz}, 4-\mathrm{H}), 6.71(\mathrm{dd}, 1 \mathrm{H}, J=8.5 \mathrm{~Hz}, J=2.5 \mathrm{~Hz}, 2-\mathrm{H}), 7.20$ (d, 1H, $J=8.5 \mathrm{~Hz}, 1-\mathrm{H}), 7.85\left(\mathrm{~s}, 1 \mathrm{H}, 5^{\prime}-\mathrm{H}\right), 8.22(\mathrm{~d}, 2 \mathrm{H}, J=9.0 \mathrm{~Hz}, 3$ "- and 5"-H), 8.72 (d, 2H, $J$ $=9.0 \mathrm{~Hz}, 2$ "'- and 6 "-H). ${ }^{13} \mathrm{C}$ NMR $\left(\delta, \mathrm{ppm}, \mathrm{CDCl}_{3}\right): 17.1(\mathrm{C}-18), 22.7,25.9,28.0,29.0,29.8$, 31.2, 38.9, 42.0, 43.5, 46.4 (C-13), 47.0 (4'-CH2), 78.8 (C-17), 111.5 (C-2), 113.8 (C-4), 114.0 (C-1'), 123.5 (C-2" and -6") 126.3 (C-5'), 130.9 (C-3" and -5"), 135.0 (C-10), 137.8 (C-5), 141.5 (C-4”), 150.6 (C-4'), $157.5(\mathrm{C}-3), 164.6(\mathrm{C}=\mathrm{O})$.

\subsubsection{3-Methoxy-16a-(4'-hydroxymethyl-1 'H-1',2',3'-triazol-1'-yl)methylestra-1,3,5(10)-trien- $17 a-o l(24 f)$}

Compound 24e (274 mg, $0.5 \mathrm{mmol})$ was dissolved in methanol $(10 \mathrm{ml})$ containing $\mathrm{NaOCH}_{3}(14$ $\mathrm{mg}, 0.25 \mathrm{mmol}$ ), and the solution was allowed to stand for $24 \mathrm{~h}$. It was then diluted with water, and the precipitate separating out was filtered off and recrystallized from a mixture of acetone/hexane to afford $\mathbf{2 4 f}(187 \mathrm{mg}, 94 \%)$ as a white crystalline product. Mp: $149-150{ }^{\circ} \mathrm{C} ; R_{\mathrm{f}}$ $=0.25$ (ss B). (Found C, 69.55; H, 7.95. $\mathrm{C}_{23} \mathrm{H}_{31} \mathrm{~N}_{3} \mathrm{O}_{3}$ (397.51) requires $\mathrm{C}, 69.49 ; \mathrm{H}, 7.86 \%$ ). ${ }^{1} \mathrm{H}$ $\operatorname{NMR}\left(\delta, \operatorname{ppm}, \mathrm{CDCl}_{3}\right): 0.74\left(\mathrm{~s}, 3 \mathrm{H}, 18-\mathrm{H}_{3}\right), 2.85\left(\mathrm{~m}, 2 \mathrm{H}, 6-\mathrm{H}_{2}\right), 3.62(\mathrm{~d}, 1 \mathrm{H}, J=4.0 \mathrm{~Hz}, 17-\mathrm{H})$, $3.77\left(\mathrm{~s}, 3 \mathrm{H}, 3-\mathrm{OCH}_{3}\right), 4.39\left(\mathrm{~m}, 1 \mathrm{H}, 16 \mathrm{a}-\mathrm{H}_{2}\right), 4.64\left(\mathrm{~m}, 1 \mathrm{H}, 16 \mathrm{a}-\mathrm{H}_{2}\right), 6.63(\mathrm{~s}, 1 \mathrm{H}, 4-\mathrm{H}), 6.71(\mathrm{~d}, 1 \mathrm{H}$, $J=8.5 \mathrm{~Hz}, 2-\mathrm{H}), 7.21(\mathrm{~d}, 1 \mathrm{H}, J=8.5 \mathrm{~Hz}, 1-\mathrm{H}), 7.77\left(\mathrm{~s}, 1 \mathrm{H}, 5^{\prime}-\mathrm{H}\right) .{ }^{13} \mathrm{C} \mathrm{NMR}\left(\delta, \mathrm{ppm}, \mathrm{CDCl}_{3}\right)$ : 11.9 (C-18), 26.0, 28.0, 28.9, 31.3, 31.9, 33.8 (C-13), 38.9, 41.9, 43.5, 46.4 (4'- $\left.\mathrm{CH}_{2}\right), 46.9,51.0$ (C-16a), 55.2 (3- $\left.\mathrm{OCH}_{3}\right), 78.6$ (C-17), 111.5 (C-2), 113.8 (C-4), 123.4 (C-5'), 126.3 (C-1), 132.5 (C-10), 137.8 (C-5), 157.4 (C-3).

2.3.25. 3-Benzyloxy-16 $\beta$-(4'-cyclopropyl-1 'H-1',2',3'-triazol-1'-yl)methylestra-1,3,5(10)-trien$17 \beta-o l(25 a)$ 
Compound 17 (420 mg, $1 \mathrm{mmol})$ and cyclopropylacetylene ( $2 \mathrm{mmol}, 0.22 \mathrm{ml})$ were used for the synthesis as described in Section 2.3. The crude product was chromatographed on silica gel with ethyl acetate $/ \mathrm{CH}_{2} \mathrm{Cl}_{2}(1: 99 \mathrm{v} / \mathrm{v})$ to yield pure $25 \mathrm{a}$ (394 $\left.\mathrm{mg}, 84 \%\right)$ as a white solid. Mp: 278-280 ${ }^{\circ} \mathrm{C} ; R_{\mathrm{f}}=0.35$ (ss B). (Found $\mathrm{C}, 77.16 ; \mathrm{H}, 7.62 . \mathrm{C}_{31} \mathrm{H}_{37} \mathrm{~N}_{3} \mathrm{O}_{2}$ (483.64) requires $\mathrm{C}, 76.98 ; \mathrm{H}$, 7.71\%). ${ }^{1} \mathrm{H}$ NMR ( $\delta$, ppm, $\left.\mathrm{CDCl}_{3}\right): 0.80$ (s, 3H, 18- $\left.\mathrm{H}_{3}\right), 0.86$ and $0.97(2 \mathrm{x} \mathrm{m}, 2$ x 2H, 2"- and 3"H), $2.83\left(\mathrm{~m}, 2 \mathrm{H}, 6-\mathrm{H}_{2}\right), 3.93(\mathrm{~d}, J=9.5 \mathrm{~Hz}, 1 \mathrm{H}, 17-\mathrm{H}), 4.21\left(\mathrm{~m}, 1 \mathrm{H}, 16 \mathrm{a}-\mathrm{H}_{2}\right), 4.64(\mathrm{~m}, 1 \mathrm{H}, 16 \mathrm{a}-$ $\left.\mathrm{H}_{2}\right), 5.03$ (s, 2H, Bn- $\left.\mathrm{H}_{2}\right), 6.71(\mathrm{~s}, 1 \mathrm{H}, 4-\mathrm{H}), 6.78(\mathrm{~d}, 1 \mathrm{H}, J=8.5 \mathrm{~Hz}, 2-\mathrm{H}), 7.20(\mathrm{~d}, 1 \mathrm{H}, J=8.5 \mathrm{~Hz}$, 1-H), 7.31 (t, 1H, $\left.J=7.0 \mathrm{~Hz}, 4^{\prime}-\mathrm{H}\right), 7.38$ (t, 2H, $J=7.0 \mathrm{~Hz}, 3^{\prime}-$ and 5'-H), 7.43 (d, 2H, $J=7.0$ $\mathrm{Hz}, 2^{\prime}$ - and 6'-H). ${ }^{13} \mathrm{C}$ NMR ( $\delta$, ppm, $\mathrm{CDCl}_{3}$ ): 7.8 (C-2" and -3"), 12.3 (C-18), 26.2, 27.4, 29.7,

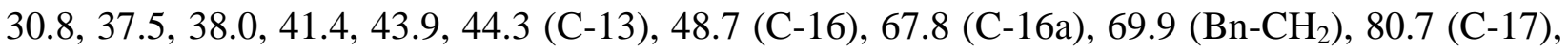
112.3 (C-2), 114.8 (C-4), 126.3 (C-1), 127.4 (C-2' and -6'), 127.8 (C-4'), 128.5 (C-3' and C-5'), 132.7 (C-10), 137.3 (C-1'), 137.8 (C-5), 156.8 (C-3).

2.3.26. 3-Benzyloxy-163-(4'-cyclopentyl-1 'H-1 ',2',3'-triazol-1'-yl)methylestra-1,3,5(10)-trien$17 \beta$-ol (25b)

Compound $17(420 \mathrm{mg}, 1 \mathrm{mmol})$ and cyclopentylacetylene $(2 \mathrm{~mol}, 0.22 \mathrm{ml})$ were used for the synthesis as described in Section 2.3. The crude product was chromatographed on silica gel with ethyl acetate $/ \mathrm{CH}_{2} \mathrm{Cl}_{2}(1: 99 \mathrm{v} / \mathrm{v})$ to yield pure $25 \mathrm{~b}(350 \mathrm{mg}, 68 \%)$ as a white solid. Mp: 288-290 ${ }^{\circ} \mathrm{C} ; R_{\mathrm{f}}=0.38$ (ss B). Found $\mathrm{C}, 77.58 ; \mathrm{H}, 7.92 . \mathrm{C}_{33} \mathrm{H}_{41} \mathrm{~N}_{3} \mathrm{O}_{2}(511.70)$ requires $\mathrm{C}, 77.46 ; \mathrm{H}, 8.08 \%$ ).

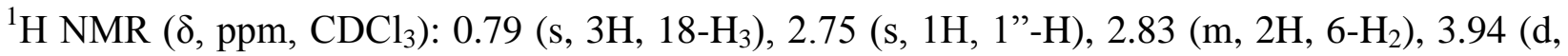
$1 \mathrm{H}, J=9.5 \mathrm{~Hz}, 17-\mathrm{H}), 4.24\left(\mathrm{~m}, 1 \mathrm{H}, 16-\mathrm{H}_{2}\right), 4.67\left(\mathrm{~m}, 1 \mathrm{H}, 16-\mathrm{H}_{2}\right), 5.03$ (s., $\left.2 \mathrm{H}, \mathrm{Bn}-\mathrm{H}_{2}\right), 6.71$ (s, 1H, 4-H), 6.78 (d, 1H, $J=8.5 \mathrm{~Hz}, 2-\mathrm{H}), 7.19$ (d, 1H, $J=8.5 \mathrm{~Hz}, 1-\mathrm{H}), 7.31$ (t, $1 \mathrm{H}, J=7.5 \mathrm{~Hz}, 4$ 'H), $7.38\left(\mathrm{t}, 2 \mathrm{H}, J=7.5 \mathrm{~Hz}, 3^{\prime}-\right.$ and $\left.5^{\prime}-\mathrm{H}\right), 7.42\left(\mathrm{~d}, 2 \mathrm{H}, J=7.5 \mathrm{~Hz}, 2^{\prime}-\right.$ and $\left.6^{\prime}-\mathrm{H}\right) .{ }^{13} \mathrm{C}$ NMR $(\delta$, ppm, $\mathrm{CDCl}_{3}$ ): 12.3 (C-18), 25.1 (C-3" and -4"), 26.2, 27.5, 29.7, 30.8, 34.3 (C-2" and -5"), 37.5,

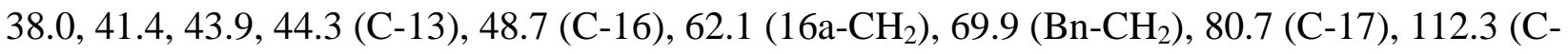
2), 114.8 (C-4), 126.3 (C-1), 127.4 (C-2' and -6'), 127.8 (C-4'), 128.5 (C-3' and -5'), 132.7 (C10), 137.3 (C-1'), 137.8 (C-5), 156.8 (C-3).

2.3.27. 3-Benzyloxy-16ß-(4'-cyclohexyl-1'H-1',2',3'-triazol-1'-yl)methylestra-1,3,5(10)-trien$17 \beta-o l(25 c)$ 
Compound $17(420 \mathrm{mg}, 1 \mathrm{mmol})$ and cyclohexylacetylene $(2 \mathrm{mmol}, 0.22 \mathrm{ml})$ were used for the synthesis as described in Section 2.3. The crude product was chromatographed on silica gel with ethyl acetate/ $\mathrm{CH}_{2} \mathrm{Cl}_{2}(1: 99, \mathrm{v} / \mathrm{v})$ to yield pure $25 \mathrm{c}$ (146 mg, 28\%) as a white solid. Mp: 214-216 ${ }^{\circ} \mathrm{C} ; R_{\mathrm{f}}=0.38$ (ss B). (Found $\mathrm{C}, 77.43 ; \mathrm{H}, 8.36 . \mathrm{C}_{34} \mathrm{H}_{43} \mathrm{~N}_{3} \mathrm{O}_{2}(525.72$ ) requires $\mathrm{C}, 77.68 ; \mathrm{H}, 8.24 \%$ ). ${ }^{1} \mathrm{H}$ NMR $\left(\delta, \mathrm{ppm}, \mathrm{CDCl}_{3}\right)$ : 0.79 (s, 3H, 18- $\left.\mathrm{H}_{3}\right), 2.79$ (m, 4H, 3"- and 5"-H), $3.94(\mathrm{~d}, J=9.5 \mathrm{~Hz}$, 1H, 17-H), 4.25 (m, 1H, 16a- $\left.\mathrm{H}_{2}\right), 4.67\left(\mathrm{~m}, 1 \mathrm{H}, 16 \mathrm{a}-\mathrm{H}_{2}\right), 5.03\left(\mathrm{~s}, 2 \mathrm{H}, \mathrm{Bn}-\mathrm{H}_{2}\right), 6.71(\mathrm{~s}, 1 \mathrm{H}, 4-\mathrm{H})$, $6.78(\mathrm{~d}, 1 \mathrm{H}, \mathrm{J}=8.5 \mathrm{~Hz}, 2-\mathrm{H}), 7.19$ (d, 1H, J = 8.5 Hz, 1-H), 7.32 (d, 1H, $\left.J=7.0 \mathrm{~Hz}, 4^{\prime}-\mathrm{H}\right), 7.38$ (t, $2 \mathrm{H}, J=7.0 \mathrm{~Hz}, 3^{\prime}-$ and $\left.5^{\prime}-\mathrm{H}\right), 7.42\left(\mathrm{~d}, 2 \mathrm{H}, J=7 \mathrm{~Hz}, 2^{\prime}-\right.$ and $\left.6^{\prime}-\mathrm{H}\right) .{ }^{13} \mathrm{C}$ NMR $\left(\delta, \mathrm{ppm}, \mathrm{CDCl}_{3}\right)$ : 12.3 (C-18), 26.0 (C-4"), 26.1 (C-3" and -5"), 26.2, 27.5, 29.7, 30.8 (C-2" and -6"), 33.0 (C-1"),

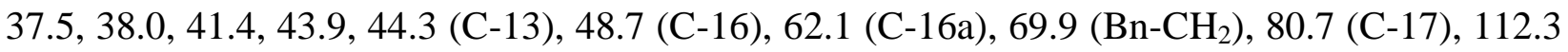
(C-2), 114.8 (C-4), 126.3 (C-1), 127.4 (C-2' and -6'), 127.8 (C-4'), 128.5 (C-3' and -5'), 132.7 (C-10), 137.3 (C-1'), 137.8 (C-5), 157.8 (C-3).

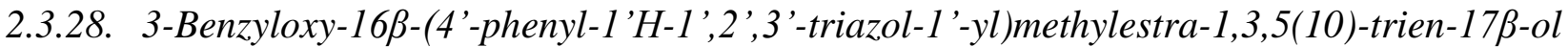
(25d)

Compound $17(420 \mathrm{mg}, 1 \mathrm{mmol})$ and phenylacetylene $(2 \mathrm{mmol}, 0.22 \mathrm{ml})$ were used for the synthesis as described in Section 2.3. The crude product was chromatographed on silica gel with ethyl acetate $/ \mathrm{CH}_{2} \mathrm{Cl}_{2}(5: 95 \mathrm{v} / \mathrm{v})$ to yield pure $25 \mathrm{~d}$ (391 $\left.\mathrm{mg}, 75 \%\right)$ as a white solid. Mp: 202-204 ${ }^{\circ} \mathrm{C} ; R_{\mathrm{f}}=0.45$ (ss B). (Found C, 78.73; H, 6.98. $\mathrm{C}_{34} \mathrm{H}_{37} \mathrm{~N}_{3} \mathrm{O}_{2}(519.68)$ requires $\mathrm{C}, 78.58 ; \mathrm{H}, 7.18 \%$ ). ${ }^{1} \mathrm{H}$ NMR $\left(\delta\right.$, ppm, $\left.\mathrm{C}_{6} \mathrm{D}_{6}\right): 0.68\left(\mathrm{~s}, 3 \mathrm{H}, 18-\mathrm{H}_{3}\right), 2.69\left(\mathrm{~m}, 2 \mathrm{H}, 6-\mathrm{H}_{2}\right), 3.43(\mathrm{dd}, J=9.5 \mathrm{~Hz}, J=4 \mathrm{~Hz}$, $1 \mathrm{H}, 17-\mathrm{H}), 3.77\left(\mathrm{dd}, 1 \mathrm{H}, \mathrm{J}=13.5 \mathrm{~Hz}, \mathrm{~J}=7.0 \mathrm{~Hz}, 16 \mathrm{a}-\mathrm{H}_{2}\right), 4.29(\mathrm{dd}, 1 \mathrm{H}, \mathrm{J}=13.5 \mathrm{~Hz}, \mathrm{~J}=7.0 \mathrm{~Hz}$, 16a- $\mathrm{H}_{2}$ ), 4.83 (s, 2H, Bn-H $\mathrm{H}_{2}$ ), 6.79 (s, 1H, 4-H), 6.87 (d, 1H, $\left.J=8.0 \mathrm{~Hz}, 2-\mathrm{H}\right), 7.02$ (s, 1H, 1-H), 7.08 (t, $\left.1 \mathrm{H}, J=7.5 \mathrm{~Hz}, 4^{\prime}-\mathrm{H}\right), 7,26$ (t, $2 \mathrm{H}, J=7.5 \mathrm{~Hz}, 3^{\prime}-$ and $\left.5^{\prime}-\mathrm{H}\right), 7.32\left(\mathrm{~d}, 2 \mathrm{H}, J=7.5 \mathrm{~Hz}, 2^{\prime}-\right.$ and 6'-H), $8.01(\mathrm{~d}, 2 \mathrm{H}, J=7.5 \mathrm{~Hz}, 2$ '- and 6"-H).

2.3.29. 3-Benzyloxy-163-[4'-(4',-nitro-benzoyloxymethyl)-1 'H-1',2',3'-triazol-1'-yl]methyestra1,3,5(10)-trien-17 $\beta$-ol (25e)

Compound 17 (420 mg, $1 \mathrm{mmol}$ ) and propargyl 4-nitrobenzoate ( $2 \mathrm{mmol}, 210 \mathrm{mg}$ ) were used for the synthesis as described in Section 2.3. The crude product was chromatographed on silica gel ethyl acetate $/ \mathrm{CH}_{2} \mathrm{Cl}_{2}(5: 95 \mathrm{v} / \mathrm{v})$ to yield pure $25 \mathbf{e}(480 \mathrm{mg}, 77 \%)$ as a yellow solid. Mp: 187-189 ${ }^{\circ} \mathrm{C} ; R_{\mathrm{f}}=0.45$ (ss B). (Found C, 69.32; 5.98. $\mathrm{C}_{36} \mathrm{H}_{38} \mathrm{~N}_{4} \mathrm{O}_{6}$ (622.71) requires $\mathrm{C}, 69.44 ; \mathrm{H}, 6.15 \%$ ). 
${ }^{1} \mathrm{H}$ NMR $\left(\delta, \mathrm{ppm}, \mathrm{CDCl}_{3}\right): 0.80\left(\mathrm{~s}, 3 \mathrm{H}, 18-\mathrm{H}_{3}\right), 2.82\left(\mathrm{~m}, 2 \mathrm{H}, 6-\mathrm{H}_{2}\right), 3.94(\mathrm{~d}, J=10.0 \mathrm{~Hz}, 1 \mathrm{H}, 17-$ H), $4.32\left(\mathrm{dd}, 1 \mathrm{H}, \mathrm{J}=13.0 \mathrm{~Hz}, \mathrm{~J}=6.0 \mathrm{~Hz}, 16 \mathrm{a}-\mathrm{H}_{2}\right), 4.72\left(\mathrm{t}, 1 \mathrm{H}, \mathrm{J}=6.0 \mathrm{~Hz}, 16 \mathrm{a}-\mathrm{H}_{2}\right), 5.03(\mathrm{~s}, 2 \mathrm{H}$, Bn- $\left.\mathrm{H}_{2}\right), 5.52(\mathrm{~s}, 2 \mathrm{H}$, triazol-H), $6.71(\mathrm{~s}, 1 \mathrm{H}, 4-\mathrm{H}), 6.78(\mathrm{~d}, 1 \mathrm{H}, J=8.5 \mathrm{~Hz}, 2-\mathrm{H}), 7.19(\mathrm{~d}, 1 \mathrm{H}, J=$ $8.5 \mathrm{~Hz}, 1-\mathrm{H}), 7.32$ (t, 1H, $\left.J=7.0 \mathrm{~Hz}, 4^{\prime}-\mathrm{H}\right), 7.38$ (t, $J=7.5 \mathrm{~Hz}, 2 \mathrm{H}, 3^{\prime}{ }^{\prime}-$ and $\left.5^{\prime}-\mathrm{H}\right), 7.42$ (d, $J=$ $7.5 \mathrm{~Hz}, 2 \mathrm{H}, 2$ '- and 6'-H), 8.22 (d, $J=8 \mathrm{~Hz}, 2 \mathrm{H}, 3$ "'- and 5'-H), 8.27 (d, $J=8 \mathrm{~Hz}, 2 \mathrm{H}, 2$ '- and 6"-H). ${ }^{13} \mathrm{C}$ NMR ( $\delta$, ppm, $\mathrm{CDCl}_{3}$ ): 12.3 (C-18), 26.2, 27.4, 29.7, 30.8, 37.4, 38.0, 41.2, 43.8, 44.4

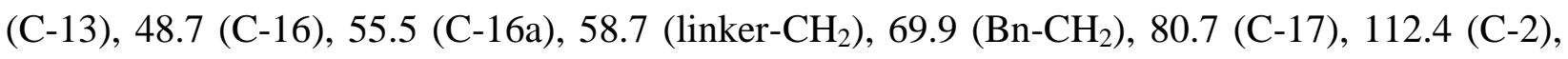
114.8 (C-4), 123.5 (C-2' and -6'), 126.3 (C-1), 127.4 (C-2" and -6”), 127.8 (C-4'), 128.5 (C-3" and -5”), 130.9 (C-3' and -5'), 132.5 (C-10), 135.1 (C-1”), 137.3 (C-1'), 137.8 (C-5), 150.7 (C$4 "), 156.8(\mathrm{C}-3), 164.6(\mathrm{C}=\mathrm{O})$.

2.3.30. 3-Benzyloxy-163-(4'-hydroxymethyl-1'H-1',2',3'-triazol-1'-yl)methylestra-1,3,5(10)trien-17 $\beta$-ol (25f)

Compound 25e (210 mg, $0.5 \mathrm{mmol})$ was dissolved in methanol (10 ml) containing $\mathrm{NaOCH}_{3}(14$ $\mathrm{mg}, 0.25 \mathrm{mmol}$ ), and the solution was allowed to stand for $24 \mathrm{~h}$. It was then diluted with water, and the precipitate separating out was filtered off and recrystallized from methanol to afford $\mathbf{2 5 f}$ (232 mg, 98\%) as a white crystalline product. Mp: $283-285{ }^{\circ} \mathrm{C} ; R_{\mathrm{f}}=0.25$ (ss B). (Found C, 73.42; $\mathrm{H}, 7.35 . \mathrm{C}_{29} \mathrm{H}_{35} \mathrm{~N}_{3} \mathrm{O}_{3}$ (473.61) requires $\left.\mathrm{C}, 73.54 ; \mathrm{H}, 7.45 \%\right)$. ${ }^{1} \mathrm{H} \mathrm{NMR}\left(\delta\right.$, ppm, DMSO-d $\left.\mathrm{d}_{6}\right)$ : $0.77\left(\mathrm{~s}, 3 \mathrm{H}, 18-\mathrm{H}_{3}\right), 3.77\left(\mathrm{dd}, 1 \mathrm{H}, \mathrm{J}=9.5 \mathrm{~Hz}, \mathrm{~J}=3.5 \mathrm{~Hz}, 16 \mathrm{a}-\mathrm{H}_{2}\right), 4.15(\mathrm{t}, 1 \mathrm{H}, \mathrm{J}=12.5 \mathrm{~Hz}, 16 \mathrm{a}-$ $\left.\mathrm{H}_{2}\right), 5.12(\mathrm{~d}, 1 \mathrm{H}, \mathrm{J}=5.5 \mathrm{~Hz}, 17-\mathrm{H}), 6.68(\mathrm{~s}, 1 \mathrm{H}, 4-\mathrm{H}), 6.74(\mathrm{~d}, 1 \mathrm{H}, J=8.5 \mathrm{~Hz}, 2-\mathrm{H}), 7.16(\mathrm{~d}, J=$ $8.5 \mathrm{~Hz}, 1 \mathrm{H}, 1-\mathrm{H}), 7.31\left(\mathrm{~d}, 1 \mathrm{H}, J=7.0 \mathrm{~Hz}, 4^{\prime}-\mathrm{H}\right), 7.37$ (t, 2H, $J=7.0 \mathrm{~Hz}, 3^{\prime}-$ and 5'-H), 7.41 (d, $2 \mathrm{H}, J=7.0 \mathrm{~Hz} ., 2$ '- and $\left.6{ }^{\prime}-\mathrm{H}\right), 7.98$ (s, $1 \mathrm{H}$, triazol-H). ${ }^{13} \mathrm{C}$ NMR $\left(\delta, \mathrm{ppm}, \mathrm{DMSO}-\mathrm{d}_{6}\right): 12.3(\mathrm{C}-$ 18), 25.8, 26.9, 29.1, 30.0, 36.9, 37.8, 40.4, 43.4, 43.7 (C-13), 47.8 (C-16a), 55.0 (linker- $\mathrm{CH}_{2}$ ), $68.9\left(\mathrm{Bn}^{-\mathrm{CH}_{2}}\right), 79.5$ (C-17), 112.1 (C-2), 114.4 (C-4), 122.7 (triazol-CH), 126.0 (C-1), 127.4 (C2' and -6'), 127.6 (C-4'), 128.3 (C-3' and -5'), 132.3 (C-10), 137.3 (C-5), 147.6 (triazol-C), 156.0 (C-3).

2.3.31. 3-Benzyloxy-16a-(4'-cyclopropyl-1'H-1',2, '3'-triazol-1'-yl)methylestra-1,3,5(10)-trien$17 \beta$-ol (26a)

Compound 18 (420.0 mg, $1 \mathrm{mmol}$ ) and cyclopropylacetylene ( $2 \mathrm{mmol}, 0.22 \mathrm{ml})$ were used for the synthesis as described in Section 2.3. The crude product was chromatographed on silica gel with 
ethyl acetate $/ \mathrm{CH}_{2} \mathrm{Cl}_{2}(1: 99 \mathrm{v} / \mathrm{v})$ to yield pure $\mathbf{2 6 a}(310 \mathrm{mg}, 64 \%)$ as a white solid. Mp: 191-193 ${ }^{\circ} \mathrm{C} ; R_{\mathrm{f}}=0.35$ (ss B). (Found $\mathrm{C}, 76.82 ; \mathrm{H}, 7.94 . \mathrm{C}_{31} \mathrm{H}_{37} \mathrm{~N}_{3} \mathrm{O}_{2}$ (483.64) requires $\mathrm{C}, 76.98 ; \mathrm{H}$, 7.71\%). ${ }^{1} \mathrm{H}$ NMR ( $\left.\delta, \mathrm{ppm}, \mathrm{CDCl}_{3}\right): 0.83$ (s, 3H, 18- $\left.\mathrm{H}_{3}\right), 2.83\left(\mathrm{~m}, 2 \mathrm{H}, 6-\mathrm{H}_{2}\right), 3.54(\mathrm{~d}, J=7.5 \mathrm{~Hz}$, $1 \mathrm{H}, 17-\mathrm{H}), 4.35\left(\mathrm{dd}, 1 \mathrm{H}, J=13.0 \mathrm{~Hz}, J=7.5 \mathrm{~Hz}, 16 \mathrm{a}-\mathrm{H}_{2}\right), 4.44(\mathrm{dd}, 1 \mathrm{H}, J=13.0 \mathrm{~Hz}, J=7.5 \mathrm{~Hz}$, 16a- $\left.\mathrm{H}_{2}\right), 5.03$ (s, 2H, Bn-H $), 6.71(\mathrm{~s}, 1 \mathrm{H}, 4-\mathrm{H}), 6.77$ (d, 1H, $\left.J=8.5 \mathrm{~Hz}, 2-\mathrm{H}\right), 7.19$ (d, 1H, $J=$ $8.5 \mathrm{~Hz}, 1-\mathrm{H}), 7.31$ (t, $2 \mathrm{H}, J=7.5 \mathrm{~Hz}, 4^{\prime}-\mathrm{H}$ and triazol-H), 7.38 (t, $2 \mathrm{H}, J=7.5 \mathrm{~Hz}, 3^{\prime}-$ and 5 ' $-\mathrm{H}$ ), $7.42\left(\mathrm{~d}, 2 \mathrm{H}, J=7.5 \mathrm{~Hz}, 2\right.$ '- and 6'-H). ${ }^{13} \mathrm{C} \mathrm{NMR}\left(\delta, \mathrm{ppm}, \mathrm{CDCl}_{3}\right): 6.6$ (C-1"), 7.8 (C-2" and -3"), 11.8 (C-18), 26.1, 27.2, 28.2, 29.7, 36.6, 38.4, 43.9, 44.3, 44.3 (C-13), 48.3 (C-16), 54.5 (C-16a), $69.9\left(\mathrm{Bn}^{-\mathrm{CH}_{2}}\right), 85.2$ (C-17), 112.3 (C-2), 114.8 (C-4), 120.0 (triazol-CH), 126.3 (C-1), 127.4 (C2' and -6'), 127.8 (C-4'), 128.5 (C-3' and -5'), 132.6 (C-10), 137.3 (C-1'), 137.8 (C-5), 150.2 (triazol-C), 156.8 (C-3).

\subsubsection{3-Benzyloxy-16a-(4'-cyclopentyl-1'H-1',2',3'-triazol-1'-yl)methylestra-1,3,5(10)-trien-} $17 \beta-o l(26 b)$

Compound 18 (420 mg, $1 \mathrm{mmol})$ and cyclopentylacetylene $(2 \mathrm{mmol}, 0.22 \mathrm{ml})$ were used for the synthesis as described in Section 2.3. The crude product was chromatographed on silica gel with ethyl acetate/ $\mathrm{CH}_{2} \mathrm{Cl}_{2}(1: 99 \mathrm{v} / \mathrm{v})$ to yield pure $\mathbf{2 6 b}(442 \mathrm{mg}, 86 \%)$ as a white solid. Mp: 268-270 ${ }^{\circ} \mathrm{C} ; R_{\mathrm{f}}=0.36$ (ss B). (Found C, 77.52; H, 7.93. $\mathrm{C}_{33} \mathrm{H}_{41} \mathrm{~N}_{3} \mathrm{O}_{2}(511.70$ ) requires $\mathrm{C}, 77.46 ; \mathrm{H}, 8.08 \%$ ). ${ }^{1} \mathrm{H}$ NMR ( $\delta$, ppm, $\mathrm{CDCl}_{3}$ ): 0.83 (s, 3H, 18- $\left.\mathrm{H}_{3}\right), 2.83$ (m, 2H, 6- $\left.\mathrm{H}_{2}\right), 3.19$ (s, 1H, 1"-H), 3.46 (d, $1 \mathrm{H}, J=7.0 \mathrm{~Hz}, 17-\mathrm{H}), 4.42\left(\mathrm{dd}, 2 \mathrm{H}, J=22.5 \mathrm{~Hz}, J=6.5 \mathrm{~Hz}, 16-\mathrm{H}_{2}\right), 5.03\left(\mathrm{~s}, 2 \mathrm{H}, \mathrm{Bn}-\mathrm{H}_{2}\right), 6.71$ (s, 1H, 4-H), 6.76 (d, 1H, $J=8.5 \mathrm{~Hz}, 2-\mathrm{H}), 7.19$ (d, 1H, $J=8.5 \mathrm{~Hz}, 1-\mathrm{H}), 7.31$ (t, 1H, $J=7.5 \mathrm{~Hz}$, 4'-H), 7.37 (t, 3H, $J=7.5 \mathrm{~Hz}, 3^{\prime}-5^{\prime} '-\mathrm{H}$ and triazol-H), 7.42 (d, 2H, $J=7.5 \mathrm{~Hz}, 2^{\prime}-$ and 6'-H). ${ }^{13} \mathrm{C}$ NMR ( $\delta$, ppm, $\left.\mathrm{CDCl}_{3}\right): 11.9$ (C-18), 25.1 (C-3” and -4”), 26.1, 27.2, 28.3, 29.7, 33.2 (C-2” and -5”), 36.6 (2C, C-1"), 36.7, 38.4, 43.9, 44.3 (C-13), 48.4 (C-16), 54.5 (C-16a), 69.9 (Bn$\mathrm{CH}_{2}$ ), 85.2 (C-17), 112.3 (C-2), 114.8 (C-4), 126.3 (C-1), 127.4 (C-3' and -5'), 127.8 (C-4'), 128.5 (C-2' and -6'), 132.6 (C-10), 137.3 (C-1'), 137.8 (C-5), 156.7 (C-3).

\subsubsection{3-Benzyloxy-16a-(4'-cyclohexyl-1 'H-1',2',3'-triazol-1'-yl)methylestra-1,3,5(10)-trien- $17 \beta-o l(26 c)$}

Compound 18 (420 mg, $1 \mathrm{mmol})$ and cyclohexylacetylene $(2 \mathrm{mmol}, 0.22 \mathrm{ml})$ were used for the synthesis as described in Section 2.3. The crude product was chromatographed on silica gel with 
ethyl acetate $/ \mathrm{CH}_{2} \mathrm{Cl}_{2}(2.5: 77.5 \mathrm{v} / \mathrm{v})$ to yield pure 26c $(386 \mathrm{mg}, 76 \%)$ as a white solid. Mp: 261-263 ${ }^{\circ} \mathrm{C} ; R_{\mathrm{f}}=0.34$ (ss B). (Found C, 77.93; H, 8.36. $\mathrm{C}_{34} \mathrm{H}_{43} \mathrm{~N}_{3} \mathrm{O}_{2}$ (525.72) requires C, 77.68; $\mathrm{H}, 8.24 \%) .{ }^{1} \mathrm{H} \mathrm{NMR}\left(\delta, \mathrm{ppm}, \mathrm{CDCl}_{3}\right): 0.83\left(\mathrm{~s}, 3 \mathrm{H}, 18-\mathrm{H}_{3}\right), 2.83\left(\mathrm{~m}, 2 \mathrm{H}, 6-\mathrm{H}_{2}\right), 3.55(\mathrm{~d}, J=7.0$ $\mathrm{Hz}, 1 \mathrm{H}, 17-\mathrm{H}), 4.43$ (m, 2H, 16- $\left.\mathrm{H}_{2}\right), 5.03$ (s, 2H, Bn-H2), 6.71 (s, 1H, 4-H), 6.77 (d, 1H, J = 8.5 $\mathrm{Hz}, 2-\mathrm{H}), 7.19(\mathrm{~d}, 1 \mathrm{H}, J=8.5 \mathrm{~Hz}, 1-\mathrm{H}), 7.31$ (t, 2H, $J=7.0 \mathrm{~Hz}, 4$ '-H and triazol-H), $7.37(\mathrm{t}, 2 \mathrm{H}$, $J=7.0 \mathrm{~Hz} .3^{\prime}-$ and 5'-H), $7.42\left(\mathrm{~d}, 2 \mathrm{H}, J=7 \mathrm{~Hz}, 2^{\prime}-\right.$ and $\left.6{ }^{\prime}-\mathrm{H}\right) .{ }^{13} \mathrm{C}$ NMR $\left(\delta, \mathrm{ppm}, \mathrm{CDCl}_{3}\right): 11.9$ (C-18), 25.9 (C-4"), 26.1 (C-3" and -5"), 27.2, 28.3, 29.7 (C-2" and -6"), 32.9, 33.0, 36.6, 38.4, 43.9, 44.2, 44.3 (C-13), 48.4 (C-16), 54.5 (C-16a), 69.9 (Bn-CH $\mathrm{CH}_{2}, 85.2$ (C-17), 112.3 (C-2), 114.8 (C-4), 126.3 (C-1), 127.4 (C-2' and -6'), 127.8 (C-4'), 128.5 (C-3' and -5'), 132.6 (C-10), 137.3 (C-1'), 137.8 (C-5), 156.7 (C-3).

2.3.34. 3-Benzyloxy-16a-(4'-phenyl-1'H-1',2',3'-triazol-1'-yl)methylestra-1,3,5(10)-trien-17 3 -ol (26d)

Compound 18 (420 mg, $1 \mathrm{mmol}$ ) and phenylacetylene (2 mmol, $0.22 \mathrm{ml}$ ) were used for the synthesis as described in Section 2.3. The crude product was chromatographed on silica gel with ethyl acetate $/ \mathrm{CH}_{2} \mathrm{Cl}_{2}$ 5:95 v/v) to yield pure 26d (372 mg, 71\%) as a white solid. Mp: 132-134 ${ }^{\circ} \mathrm{C} ; R_{\mathrm{f}}=0.38$ (ss B). (Found C, 78.63; H, 6.97. $\mathrm{C}_{34} \mathrm{H}_{37} \mathrm{~N}_{3} \mathrm{O}_{2}(519.68)$ requires C, 78.58; H, 7.18\%). ${ }^{1} \mathrm{H}$ NMR $\left(\delta, p p m, \mathrm{CDCl}_{3}\right): 0.84\left(\mathrm{~s}, 3 \mathrm{H}, 18-\mathrm{H}_{3}\right), 2.83\left(\mathrm{~m}, 2 \mathrm{H}, 6-\mathrm{H}_{2}\right), 3.58(\mathrm{~d}, 1 \mathrm{H}, J=7.5 \mathrm{~Hz}, 17-$ H), $4.46\left(\mathrm{dd}, 2 \mathrm{H}, J=13.5 \mathrm{~Hz}, J=8.0 \mathrm{~Hz}, 16 \mathrm{a}-\mathrm{H}_{2}\right), 4.55(\mathrm{dd}, 1 \mathrm{H}, J=13.5 \mathrm{~Hz}, J=8.0 \mathrm{~Hz}, 16 \mathrm{a}-$ H2) 5.03 (s, 2H, Bn-H2), $6.71(\mathrm{~s}, 1 \mathrm{H}, 4-\mathrm{H}), 6.78(\mathrm{~d}, 1 \mathrm{H}, J=8.5 \mathrm{~Hz}, 2-\mathrm{H}), 7.19$ (d, 1H, $J=8.5 \mathrm{~Hz}$, 1-H), 7.30-7.86 (m, 11H, 2'-, 6'-, 3'-, 5'-, 4'-, 2’'-, 6"'-, 3"'-, 5'-, 4"'- and triazol-H). ${ }^{13} \mathrm{C}$ NMR $(\delta$, ppm, $\left.\mathrm{CDCl}_{3}\right)$ : 11.8 (C-18), 26.1, 27.2, 28.2, 29.6, 36.5, 38.4, 43.9, 44.3, 48.3 (C-16), 54.6 (C-

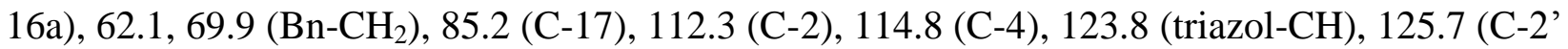
and -6'), 126.3 (C-1'), 127.4 (C-2” and -6”), 127.8 (C-4'), 128.2 (C-4), 128.5 (C-3" and -5"), 128.8 (C-3' and -5'), 130.4 (C-10), 132.6 (C-1’'), 137.3 (C-1'), 137.8 (C-5), 156.8 (C-3).

2.3.35. 3-Benzyloxy-16a-[4'-(4'”-nitro-benzoyloxymethyl)-1 'H-1 ',2',3'-triazol-1 '-yl]methylestra1,3,5(10)-trien-17 $\beta$-ol (26e)

Compound 18 (420 mg, $1 \mathrm{mmol}$ ) and propargyl 4-nitrobenzoate (2 mmol, $210 \mathrm{mg}$ ) were used for the synthesis as described in Section 2.3. The crude product was chromatographed on silica gel with ethyl acetate/ $\mathrm{CH}_{2} \mathrm{Cl}_{2}(5: 95 \mathrm{v} / \mathrm{v})$ to yield pure 26e (484 mg, 77\%) as a yellow solid. Mp: 
94-96 ${ }^{\circ} \mathrm{C} ; R_{\mathrm{f}}=0.40$ (ss B). (Found C, 69.73; H, 5.94. $\mathrm{C}_{36} \mathrm{H}_{38} \mathrm{~N}_{4} \mathrm{O}_{6}(622.71$ ) requires C, 69.44; H, 6.15\%). ${ }^{1} \mathrm{H}$ NMR $(\delta, p p m$, DMSO-d 6$): 0.70\left(\mathrm{~s}, 3 \mathrm{H}, 18-\mathrm{H}_{3}\right), 3.33\left(\mathrm{~m}, 2 \mathrm{H}, 6-\mathrm{H}_{2}\right), 4.38(\mathrm{dd}, 1 \mathrm{H}, J=$ $\left.13.5 \mathrm{~Hz}, J=9.0 \mathrm{~Hz}, 16 \mathrm{a}-\mathrm{H}_{2}\right), 4.52\left(\mathrm{dd}, 1 \mathrm{H}, J=13.5 \mathrm{~Hz}, J=5.0 \mathrm{~Hz}, 16 \mathrm{a}-\mathrm{H}_{2}\right), 4.86(\mathrm{~d}, 1 \mathrm{H}, J=5$ $\mathrm{Hz}, 17-\mathrm{H}), 5.02$ (s, 2H, Bn-H2), 5.47 (s, 2H, linker-H $\left.\mathrm{H}_{2}\right), 6.64$ (d, 1H, J = $\left.2.0 \mathrm{~Hz}, 4-\mathrm{H}\right), 6.72$ (dd, $1 \mathrm{H}, \mathrm{J}=8.5 \mathrm{~Hz}, J=2.0 \mathrm{~Hz}, 2-\mathrm{H}), 7.10(\mathrm{~d}, 1 \mathrm{H}, J=8.5 \mathrm{~Hz}, 1-\mathrm{H}), 7.31$ (t, 1H, $\left.J=7.0 \mathrm{~Hz}, 4^{\prime}-\mathrm{H}\right)$,

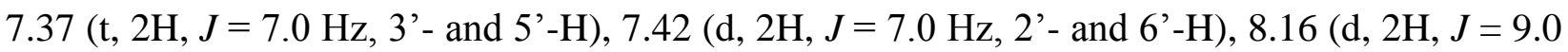
$\mathrm{Hz}, 3$ "'- and 5"-H), $8.28\left(\mathrm{~d}, 2 \mathrm{H}, J=9.0 \mathrm{~Hz}, 2\right.$ "- and 6"-H), $8.32\left(\mathrm{~s}, 1 \mathrm{H}\right.$, triazol-H). ${ }^{13} \mathrm{C}$ NMR $(\delta$, ppm, DMSO-d $\left.{ }_{6}\right): 11.7$ (C-18), 25.7, 26.6, 27.1, 29.0, 30.6, 36.4, 37.9, 43.4, 43.4 (C-13), 43.7 (C16), 53.1 (C-16a), 58.6 (linker- $\left.\mathrm{CH}_{2}\right), 68.9$ (Bn-CH$), 82.8$ (C-17), 112.1 (C-2), $114.3(\mathrm{C}-4), 123.7$ (C-2' and -6'), 125.1 (triazol-CH), 125.9 (C-1), 127.4 (C-2” and -6”), 127.5 (C-4'), 128.3 (C-3” and -5”), 130.6 (C-3' and -5'), 132.1 (C-10), 134.7 (C-1”), 137.2 (C-1'), 137.3 (C-5), 141.1 (triazol-C), 150.1 (C-4”), 155.9 (C-3), 163.9 (C=O).

2.3.36. 3-Benzyloxy-16a-(4'-hydroxymethyl-1'H-1',2',3'-triazol-1'-yl)methylestra-1,3,5(10)trien-17 $\beta$-ol (26f)

Compound 26e $(210 \mathrm{mg}, 0.5 \mathrm{mmol})$ was dissolved in methanol $(10 \mathrm{ml})$ containing $\mathrm{NaOCH}_{3}(14$ $\mathrm{mg}, 0.25 \mathrm{mmol}$ ), and the solution was allowed to stand for $24 \mathrm{~h}$. It was then diluted with water, and the precipitate separating out was filtered off and recrystallized from a mixture of acetone/hexane to afford $26 \mathbf{f}(190 \mathrm{mg}, 89 \%)$ as a white crystalline product. Mp: $152-154{ }^{\circ} \mathrm{C} ; R_{\mathrm{f}}=$ 0.20 (ss B). (Found C, 73.72; H, 7.63. $\mathrm{C}_{29} \mathrm{H}_{35} \mathrm{~N}_{3} \mathrm{O}_{3}$ (473.61) requires $\mathrm{C}, 73.54 ; \mathrm{H}, 7.45 \%$ ). ${ }^{1} \mathrm{H}$ NMR ( $\delta$, ppm, DMSO-d $\left.)_{6}\right): 0.71\left(\mathrm{~s}, 3 \mathrm{H}, 18-\mathrm{H}_{3}\right), 2.73\left(\mathrm{~m}, 2 \mathrm{H}, 6 \mathrm{H}_{2}\right), 3.29$ (d, J = 8.0 Hz, 1H, 17H), $4.28\left(\mathrm{dd}, 2 \mathrm{H}, J=13.0 \mathrm{~Hz}, J=10.0 \mathrm{~Hz}, 16 \mathrm{a}-\mathrm{H}_{2}\right), 4.47(\mathrm{dd}, 1 \mathrm{H}, J=13.0 \mathrm{~Hz}, J=4.5 \mathrm{~Hz}, 16 \mathrm{a}-$ $\left.\mathrm{H}_{2}\right), 4.51\left(\mathrm{~s}, 2 \mathrm{H}, \mathrm{Bn}-\mathrm{H}_{2}\right), 4.87\left(\mathrm{~s}, 1 \mathrm{H}\right.$, linker- $\left.\mathrm{H}_{2}\right), 5.03\left(\mathrm{~s}, 2 \mathrm{H}\right.$, triazol- $\left.\mathrm{H}_{2}\right), 5.15\left(\mathrm{~s}, 1 \mathrm{H}\right.$, linker- $\left.\mathrm{H}_{2}\right)$, $6.68(\mathrm{~s}, 1 \mathrm{H}, 4-\mathrm{H}), 6.74(\mathrm{~d}, 1 \mathrm{H}, J=8.5 \mathrm{~Hz}, 2-\mathrm{H}), 7.15(\mathrm{~d}, 1 \mathrm{H}, J=8.5 \mathrm{~Hz}, 1-\mathrm{H}), 7.31$ (t, $1 \mathrm{H}, J=7.0$ $\left.\mathrm{Hz}, 4^{\prime}-\mathrm{H}\right), 7.37$ (t, 2H, J=7.0 Hz, 3'- and 5'-H), 7.41 (d, 2H, J = 7.0 Hz, 2'- and 6'-H), 7.97 (s, $1 \mathrm{H}$, triazol-H). ${ }^{13} \mathrm{C}$ NMR $\left.(\delta \text {, ppm, DMSO-d })_{6}\right) 11.8$ (C-18), 25.8, 26.7, 27.3, 29.1, 36.4, 38.1,

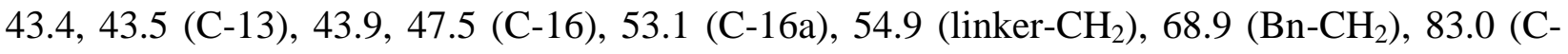
17), 112.1 (C-2), 114.4 (C-4), 122.7 (triazol-CH), 126.0 (C-1), 127.4 (C-2' and -6'), 127.6 (C-4'), 128.3 (C-3' and -5'), 132.3 (C-10), 137.3 (C-1'), 137.4 (C-5), 147.6 (triazol-C), 156.0 (C-3). 


\subsubsection{3-Benzyloxy-163-(4'-cyclopropyl-1'H-1',2',3'-triazol-1'-yl)methylestra-1,3,5(10)-trien-}

$17 a-o l(27 a)$

Compound 19 (420.0 mg, $1 \mathrm{mmol}$ ) and cyclopropylacetylene ( $2 \mathrm{mmol}, 0.22 \mathrm{ml})$ were used for the synthesis as described in Section 2.3. The crude product was chromatographed on silica gel with ethyl acetate $/ \mathrm{CH}_{2} \mathrm{Cl}_{2}(5: 95 \mathrm{v} / \mathrm{v})$ to yield pure $27 \mathbf{a}(454 \mathrm{mg}, 93 \%)$ as white crystals. Mp: 199-201 ${ }^{\circ} \mathrm{C} ; R_{\mathrm{f}}=0.38$ (ss B). (Found $\mathrm{C}, 77.15 ; \mathrm{H}, 7.62 . \mathrm{C}_{31} \mathrm{H}_{37} \mathrm{~N}_{3} \mathrm{O}_{2}$ (483.64) requires $\mathrm{C}, 76.98 ; \mathrm{H}$, 7.71\%). ${ }^{1} \mathrm{H}$ NMR $\left(\delta, \mathrm{ppm}, \mathrm{CDCl}_{3}\right): 0.77\left(\mathrm{~s}, 3 \mathrm{H}, 18-\mathrm{H}_{3}\right), 0.87$ and $0.98(2 \mathrm{x} \mathrm{s}, 2$ x $2 \mathrm{H}, 2$ "'- and 3"$\left.\mathrm{H}_{2}\right), 2.05$ (s, 1H, 1"-H), $2.84\left(\mathrm{~m}, 2 \mathrm{H}, 6-\mathrm{H}_{2}\right), 3.66(\mathrm{~s}, 1 \mathrm{H}, 17-\mathrm{H}), 4.42\left(\mathrm{~m}, 2 \mathrm{H}, 16 \mathrm{a}-\mathrm{H}_{2}\right), 5.03$ (s, 2H, Bn-H $)_{2}, 6.71(\mathrm{~s}, 1 \mathrm{H}, 4-\mathrm{H}), 6.78(\mathrm{~d}, 1 \mathrm{H}, J=8.5 \mathrm{~Hz}, 2-\mathrm{H}), 7.21(\mathrm{~d}, 1 \mathrm{H}, J=8.5 \mathrm{~Hz}, 1-\mathrm{H}), 7.31$ (t, $\left.1 \mathrm{H}, J=7.0 \mathrm{~Hz}, 4^{\prime}-\mathrm{H}\right), 7.38$ (t, 2H, $J=7.0 \mathrm{~Hz}, 3^{\prime}{ }^{\prime}-$ and $\left.5^{\prime}-\mathrm{H}\right), 7.43$ (d, 2H, $J=7.0 \mathrm{~Hz}, 2^{\prime}-$ and 6'-H). ${ }^{13} \mathrm{C}$ NMR ( $\delta$, ppm, $\mathrm{CDCl}_{3}$ ): 6.7 (C-1"), 7.7 (C-2” and -3"), 17.9 (C-18), 25.9, 27.9, 29.7,

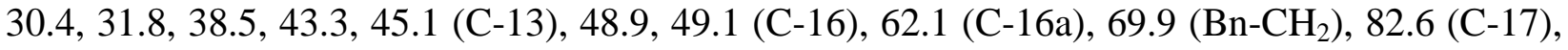
112.3 (C-2), 114.8 (C-4), 126.3 (C-1), 127.4 (C-2' and -6'), 127.8 (C-4'), 128.5 (C-3' and -5'), 132.7 (C-10), 137.3 (C-1'), 137.9 (C-5), 156.7 (C-3).

2.3.38. 3-Benzyloxy-16ß-(4'-cyclopentyl-1'H-1',2',3'-triazol-1'-yl)methylestra-1,3,5(10)-trien$17 a-o l(27 b)$

Compound 19 (420 mg, $1 \mathrm{mmol}$ ) and cyclopentylacetylene ( $2 \mathrm{mmol}, 0.22 \mathrm{ml})$ were used for the synthesis as described in Section 2.3. The crude product was chromatographed on silica gel with ethyl acetate/ $\mathrm{CH}_{2} \mathrm{Cl}_{2}(5: 95 \mathrm{v} / \mathrm{v})$ to yield pure $27 \mathrm{~b}(408 \mathrm{mg}, 79 \%)$ as white crystalline. Mp: 220-222 ${ }^{\circ} \mathrm{C} ; R_{\mathrm{f}}=0.40$ (ss B). (Found C, 77.32; H, 7.93. $\mathrm{C}_{33} \mathrm{H}_{41} \mathrm{~N}_{3} \mathrm{O}_{2}(511.70$ ) requires C, 77.46; H, 8.08\%). ${ }^{1} \mathrm{H}$ NMR ( $\delta$, ppm, $\mathrm{CDCl}_{3}$ ): 0.76 (s, 3H, 18- $\left.\mathrm{H}_{3}\right), 2.84$ (m, 2H, 6- $\left.\mathrm{H}_{2}\right), 3.20$ (s, 1H, 1"H), $3.67(\mathrm{~s}, 1 \mathrm{H}, 17-\mathrm{H}), 4.43\left(\mathrm{~m}, 2 \mathrm{H}, 16 \mathrm{a}-\mathrm{H}_{2}\right), 5.03\left(\mathrm{~s}, 2 \mathrm{H}, \mathrm{Bn}-\mathrm{H}_{2}\right), 6.72(\mathrm{~s}, 1 \mathrm{H}, 4-\mathrm{H}), 6.78(\mathrm{dd}$, $1 \mathrm{H}, J=8.5 \mathrm{~Hz}, J=2.0 \mathrm{~Hz}, 2-\mathrm{H}), 7.21(\mathrm{~d}, 1 \mathrm{H}, J=8.5 \mathrm{~Hz}, 1-\mathrm{H}), 7.31\left(\mathrm{t}, 1 \mathrm{H}, J=7.0 \mathrm{~Hz}, 4^{\prime}-\mathrm{H}\right)$, 7.38 (t, 3H, $J=7.0 \mathrm{~Hz}, 3^{\prime}-$ and $5^{\prime}-\mathrm{H}$, triazol-H), 7.43 (d, $2 \mathrm{H}, J=7.0 \mathrm{~Hz}, 2^{\prime}-$ and $\left.6 '-\mathrm{H}\right) .{ }^{13} \mathrm{C}$ NMR ( $\delta$, ppm, $\mathrm{CDCl}_{3}$ ): 18.0 (C-18), 25.1 (C-3" and -5"), 25.9, 28.0, 29.7, 30.4, 31.8 (C-2" and -6"), 33.2, 36.7, 38.5, 43.3, 45.1 (C-13), 48.9 (C-16), 49.1 (C-1”), 54.3 (C-16a), 69.9 (Bn- $\left.\mathrm{CH}_{2}\right), 82.6$ (C-17), 112.3 (C-2), 114.8 (C-4), 126.3 (C-1), 127.4 (C-2' and -6'), 127.8 (C-4'), 128.5 (C-3' and -5'), 132.7 (C-10), 137.3 (C-1'), 137.9 (C-5), 156.7 (C-3). 


\subsubsection{3-Benzyloxy-16ß-(4'-cyclohexyl-1'H-1',2',3'-triazol-1'-yl)methylestra-1,3,5(10)-trien-}

$17 a-o l(27 c)$

Compound 19 (420 mg, $1 \mathrm{mmol})$ and cyclohexylacetylene $(2 \mathrm{mmol}, 0.22 \mathrm{ml})$ were used for the synthesis as described in Section 2.3. The crude product was chromatographed on silica gel with ethyl acetate $/ \mathrm{CH}_{2} \mathrm{Cl}_{2}(5: 95 \mathrm{v} / \mathrm{v})$ to yield pure $27 \mathrm{c}(360 \mathrm{mg}, 68 \%)$ as white crystalline product. Mp: $243-245{ }^{\circ} \mathrm{C} ; R_{\mathrm{f}}=0.38$ (ss B). (Found C, 77.54; H, 8.38. $\mathrm{C}_{34} \mathrm{H}_{43} \mathrm{~N}_{3} \mathrm{O}_{2}$ (525.72) requires C, 77.68; H, 8.24\%). ${ }^{1} \mathrm{H}$ NMR ( $\delta$, ppm, $\mathrm{CDCl}_{3}$ ): 0.75 (s, 3H, 18- $\left.\mathrm{H}_{3}\right), 2.84\left(\mathrm{~m}, 2 \mathrm{H}, 6-\mathrm{H}_{2}\right), 3.68$ (s, $1 \mathrm{H}, 17-\mathrm{H}), 4.44\left(\mathrm{~m}, 2 \mathrm{H}, 16 \mathrm{a}-\mathrm{H}_{2}\right), 5.03\left(\mathrm{~s}, 2 \mathrm{H}, \mathrm{Bn}-\mathrm{H}_{2}\right), 6.72(\mathrm{~s}, 1 \mathrm{H}, 4-\mathrm{H}), 6.78(\mathrm{~d}, 1 \mathrm{H}, J=8.5 \mathrm{~Hz}$, 2-H), $7.21(\mathrm{~d}, 1 \mathrm{H}, J=8.5 \mathrm{~Hz}, 1-\mathrm{H}), 7.32\left(\mathrm{t}, 1 \mathrm{H}, J=7.0 \mathrm{~Hz}, 4^{\prime}-\mathrm{H}\right), 7.38$ (t, $3 \mathrm{H}, J=7.0 \mathrm{~Hz}, 3^{\prime}$ ' and $5^{\prime}-\mathrm{H}$, triazol-H), 7.43 (d, $2 \mathrm{H}, J=7.0 \mathrm{~Hz}, 2^{\prime}-$ and $\left.6{ }^{\prime}-\mathrm{H}\right) .{ }^{13} \mathrm{C} \mathrm{NMR}\left(\delta, \mathrm{ppm}, \mathrm{CDCl}_{3}\right)$ : $17.9(\mathrm{C}-18)$, 25.9 (C-4"), 26.0, 26.1 (C-3" and -5"), 27.9, 29.7, 30.4, 31.8 (C-2" and -6"), 32.1, 32.9 (C-1"), 38.5, 43.3, 45.1 (C-13), 48.9, 49.1 (C-16), 62.1 (C-16a), 69.9 (Bn- $\left.\mathrm{CH}_{2}\right), 82.5$ (C-17), 112.3 (C-2), 114.7 (C-4), 126.3 (C-1), 127.4 (C-2' and -6'), 127.8 (C-4'), 128.5 (C-3' and -5'), 132.7 (C-10), 137.2 (C-1'), 137.9 (C-5), 156.7 (C-3).

2.3.40. 3-Benzyloxy-16ß-(4'-phenyl-1'H-1',2',3'-triazol-1'-yl)methylestra-1,3,5(10)-trien-17a-ol (27d)

Compound $19(420 \mathrm{mg}, 1 \mathrm{mmol})$ and phenylacetylene $(2 \mathrm{mmol}, 0.22 \mathrm{ml})$ were used for the synthesis as described in Section 2.3. The crude product was chromatographed on silica gel with ethyl acetate/ $\mathrm{CH}_{2} \mathrm{Cl}_{2}(10: 90 \mathrm{v} / \mathrm{v})$ to yield pure $27 \mathbf{d}$ (487 $\left.\mathrm{mg}, 93 \%\right)$ as white crystals. Mp: 202-204 ${ }^{\circ} \mathrm{C} ; R_{\mathrm{f}}=0.45$ (ss B). (Found C, 78.68; H, 7.38. $\mathrm{C}_{34} \mathrm{H}_{37} \mathrm{~N}_{3} \mathrm{O}_{2}(519.68$ ) requires C, 78.58; $\mathrm{H}, 7.18 \%) .{ }^{1} \mathrm{H}$ NMR $\left(\delta, \mathrm{ppm}, \mathrm{CDCl}_{3}\right): 0.79\left(\mathrm{~s}, 3 \mathrm{H}, 18-\mathrm{H}_{3}\right), 2.84\left(\mathrm{~m}, 2 \mathrm{H}, 6-\mathrm{H}_{2}\right), 3.72(\mathrm{~s}, 1 \mathrm{H}, 17-$ H), $4.48\left(\mathrm{dd}, 1 \mathrm{H}, J=13.5 \mathrm{~Hz}, J=7.5 \mathrm{~Hz}, 16 \mathrm{a}-\mathrm{H}_{2}\right), 4.56\left(\mathrm{t}, 1 \mathrm{H}, J=13.5 \mathrm{~Hz}, 16 \mathrm{a}-\mathrm{H}_{2}\right), 5.03(\mathrm{~s}, 2 \mathrm{H}$, Bn- $\left.\mathrm{H}_{2}\right), 6.72(\mathrm{~s}, 1 \mathrm{H}, 4-\mathrm{H}), 6.78(\mathrm{~d}, 1 \mathrm{H}, J=8.5 \mathrm{~Hz}, 2-\mathrm{H}), 7.21$ (d, 1H, $\left.J=8.5 \mathrm{~Hz}, 1-\mathrm{H}\right), 7.33$ (t, $\left.1 \mathrm{H}, J=7.5 \mathrm{~Hz}, 4^{\prime}-\mathrm{H}\right), 7.38$ (t, $2 \mathrm{H}, J=7.5 \mathrm{~Hz}, 3^{\prime}-$ and 5'-H), 7.42 (d, $J=3.5 \mathrm{~Hz}, 4 \mathrm{H}, 2^{\prime}-$ and 6'$\mathrm{H}, 3$ "- and 5"-H), $7.84\left(\mathrm{~d}, 2 \mathrm{H}, J=7.5 \mathrm{~Hz}, 2\right.$ "- and 6"-H), $7.88\left(\mathrm{~s}, 1 \mathrm{H}\right.$, triazol-H). ${ }^{13} \mathrm{C}$ NMR $(\delta$, ppm, $\mathrm{CDCl}_{3}$ ): 17.9 (C-18), 25.9, 27.9, 29.7, 30.4, 31.8, 38.5, 43.3, 45.2 (C-13), 48.9, 49.1 (C-16), 54.6 (C-16a), 69.9 (Bn-CH ${ }_{2}$ ), 82.6 (C-17), 112.3 (C-2), 114.8 (C-4), 119.6 (triazol-CH), 125.7 (C-2' and -6'), 126.3 (C-1'), 127.4 (C-2"' and -6”), 127.8 (C-4'), 128.2 (C-4”), 128.5 (C-3"' and - 
5”), 128.8 (C-3' and -5'), 130.5 (C-10), 132.64 (C-1"), 137.3 (C-1'), 137.9 (C-5), 147.7 (triazolC); 156.8 (C-3).

\subsubsection{3-Benzyloxy-16ß-[4'-(4''-nitro-benzoyloxymethyl)-1'H-1',2',3'-triazol-1'-yl]methylestra-} 1,3,5(10)-trien-17a-ol (27e)

Compound 19 (420.0 mg, $1 \mathrm{mmol})$ and propargyl 4-nitrobenzoate $(2 \mathrm{mmol}, 210 \mathrm{mg})$ were used for the synthesis as described in Section 2.3. The crude product was chromatographed on silica gel with ethyl acetate/ $\mathrm{CH}_{2} \mathrm{Cl}_{2}(10: 90 \mathrm{v} / \mathrm{v})$ to yield pure $27 \mathrm{e}(550 \mathrm{mg}, 88 \%)$ as yellow crystals. Mp: $177-179{ }^{\circ} \mathrm{C} ; R_{\mathrm{f}}=0.48$ (ss B). (Found C, 69.55; H, 5.93. $\mathrm{C}_{36} \mathrm{H}_{38} \mathrm{~N}_{4} \mathrm{O}_{6}(622.71$ ) requires: C, 69.44; H, 6.15\%). ${ }^{1} \mathrm{H}$ NMR ( $\delta$, ppm, DMSO-d $\left.{ }_{6}\right): 0.65$ (s, 3H, 18- $\left.\mathrm{H}_{3}\right), 2.73\left(\mathrm{~m}, 2 \mathrm{H}, 6-\mathrm{H}_{2}\right), 4.40$ $\left(\mathrm{dd}, 1 \mathrm{H}, J=13.0 \mathrm{~Hz}, J=8.5 \mathrm{~Hz}, 16 \mathrm{a}-\mathrm{H}_{2}\right), 4.56\left(\mathrm{dd}, 1 \mathrm{H}, J=13.5 \mathrm{~Hz}, J=7.5 \mathrm{~Hz}, 16 \mathrm{a}-\mathrm{H}_{2}\right), 4.63$ (d, $1 \mathrm{H}, J=5.0 \mathrm{~Hz}, 17-\mathrm{H}), 5.04\left(\mathrm{~s}, 2 \mathrm{H}, \mathrm{Bn}-\mathrm{H}_{2}\right), 5.47$ (s, 2H, triazol- $\left.\mathrm{H}_{2}\right), 6.68(\mathrm{~s}, 1 \mathrm{H}, 4-\mathrm{H}), 6.74(\mathrm{~d}$, $1 \mathrm{H}, J=8.5 \mathrm{~Hz}, 2-\mathrm{H}), 7.16$ (d, 1H, $J=8.5 \mathrm{~Hz}, 1-\mathrm{H}), 7.31$ (t, 1H, $\left.J=7.0 \mathrm{~Hz}, 4^{\prime}-\mathrm{H}\right), 7.37$ (t, 2H, $J$ $=7.0 \mathrm{~Hz}, 3^{\prime}{ }^{\prime}$ and $\left.5^{\prime}-\mathrm{H}\right), 7.41\left(\mathrm{~d}, 2 \mathrm{H}, J=7.0 \mathrm{~Hz}, 2^{\prime}-\right.$ and $\left.6^{\prime}-\mathrm{H}\right), 8.18\left(\mathrm{~d}, 2 \mathrm{H}, J=8.5 \mathrm{~Hz}, 3^{\prime \prime}-\right.$ and $5 "-\mathrm{H}), 8.33\left(\mathrm{~d}, 3 \mathrm{H}, J=6 \mathrm{~Hz}, 2\right.$ "- and $6 "-\mathrm{H}$, triazol-H). ${ }^{13} \mathrm{C}$ NMR $\left(\delta, \mathrm{ppm}, \mathrm{DMSO}-\mathrm{d}_{6}\right): 17.5(\mathrm{C}-$ 18), 25.6, 27.5, 29.2, 29.6, 31.8, 38.2, 42.9, 44.5 (C-13), 48.2, 49.1 (C-16), 53.6 (C-16a), 58.7 (linker- $\left.\mathrm{CH}_{2}\right), 68.9\left(\mathrm{Bn}^{-\mathrm{CH}_{2}}\right), 80.8$ (C-17), 112.1 (C-2), 114.4 (C-4), 123.8 (C-2' and C-6'), 125.0 (triazol-CH), 126.1 (C-1), 127.4 (C-2" and -6"), 127.6 (C-4'), 128.3 (C-3" and -5"), 130.6 (C-3' and -5'), 132.3 (C-10), 134.7 (C-1"), 137.3 (C-5 and C-1'), 141.1 (triazol-C), 150.2 (C-4”), 160.0 (C-3), $163.9(\mathrm{C}=\mathrm{O})$.

2.3.42. 3-Benzyloxy-16ß-(4'-hydroxymethyl-1 'H-1',2',3'-triazol-1'-yl)methylestra-1,3,5(10)trien-17a-ol (27f)

Compound 27e (210 mg, $0.5 \mathrm{mmol})$ was dissolved in methanol $(10 \mathrm{ml})$ containing $\mathrm{NaOCH}_{3}(14$ $\mathrm{mg}, 0.25 \mathrm{mmol}$ ), and the solution was allowed to stand for $24 \mathrm{~h}$. It was then diluted with water, and the precipitate separating out was filtered off and recrystallized from methanol to afford 27e (273 mg, 99\%) as a white crystalline product. Mp: $172-174{ }^{\circ} \mathrm{C} ; R_{\mathrm{f}}=0.25$ (ss B). (Found C, 73.68; $\mathrm{H}, 7.66 . \mathrm{C}_{29} \mathrm{H}_{35} \mathrm{~N}_{3} \mathrm{O}_{3}$ (473.61) requires $\left.\mathrm{C}, 73.54 ; \mathrm{H}, 7.45 \%\right)$. ${ }^{1} \mathrm{H}$ NMR ( $\delta$, ppm, DMSO- $\mathrm{d}_{6}$ ): $0.67\left(\mathrm{~s}, 3 \mathrm{H}, 18-\mathrm{H}_{3}\right), 2.74\left(\mathrm{~m}, 2 \mathrm{H}, 6-\mathrm{H}_{2}\right), 3.43(\mathrm{~s}, 1 \mathrm{H}, 17-\mathrm{H}), 4.34\left(\mathrm{~m}, 1 \mathrm{H}, 16 \mathrm{a}-\mathrm{H}_{2}\right), 4.50(\mathrm{~m}, 3 \mathrm{H}$, 16a- $\mathrm{H}_{2}$ and $\left.\mathrm{Bn}-\mathrm{H} 2\right), 4.61$ (brs, $\left.1 \mathrm{H}, \mathrm{OH}\right), 5.04\left(\mathrm{~s}, 2 \mathrm{H}\right.$, triazol- $\left.\mathrm{H}_{2}\right), 5.16$ (brs, $\left.1 \mathrm{H}, \mathrm{OH}\right), 6.69(\mathrm{~s}, 1 \mathrm{H}$, 4-H), 6.74 (d, 1H, $J=8.5 \mathrm{~Hz}, 2-\mathrm{H}), 7.17$ (d, 1H, $J=8.5 \mathrm{~Hz}, 1-\mathrm{H}), 7.31$ (d, 1H, $\left.J=7.0 \mathrm{~Hz}, 4^{\prime}-\mathrm{H}\right)$, 
$7.37\left(\mathrm{t}, 2 \mathrm{H}, J=7.0 \mathrm{~Hz}, 3^{\prime}-\right.$ and $\left.5^{\prime}-\mathrm{H}\right), 7.41\left(\mathrm{~d}, 2 \mathrm{H}, J=7.0 \mathrm{~Hz}, 2^{\prime}-\right.$ and $\left.6^{\prime}-\mathrm{H}\right), 8.00$ (s, 1H, triazolH). ${ }^{13} \mathrm{C}$ NMR $\left(\delta\right.$, ppm, DMSO-d $\left.{ }_{6}\right): 17.5$ (C-18), 25.6, 27.5, 29.2, 29.6, 31.9, 38.2, 43.0, 44.5 (C13), 48.2, 49.1 (C-16), 53.5 (C-16a), 55.0 (linker- $\left.\mathrm{CH}_{2}\right)$, 61.6, $68.9\left(\mathrm{Bn}^{-} \mathrm{CH}_{2}\right), 80.8$ (C-17), 112.2 (C-2), 114.4 (C-4), 122.6 (triazol-CH), 126.6 (C-1), 127.4 (C-2' and -6'), 127.6 (C-4'), 128.3 (C3' and -5'), 132.4 (C-10), 137.3 (C-5 and C-1'), 147.6 (triazol-C), 156.0 (C-3).

2.3.43. 3-Benzyloxy-16a-(4'-cyclopropyl-1'H-1 ',2',3'-triazol-1'-yl)methylestra-1,3,5(10)-trien$17 a-o l(28 a)$

Compound 20 (420.0 mg, $1 \mathrm{mmol}$ ) and cyclopropylacetylene ( $2 \mathrm{mmol}, 0.22 \mathrm{ml})$ were used for the synthesis as described in Section 2.3. The crude product was chromatographed on silica gel with ethyl acetate/ $\mathrm{CH}_{2} \mathrm{Cl}_{2}(1: 99 \mathrm{v} / \mathrm{v})$ to yield pure 28a (305 mg, 63\%) as white crystals. Mp: 143-144 ${ }^{\circ} \mathrm{C} ; R_{\mathrm{f}}=0.40$ (ss B). (Found C, 77.15; H, 7.53. $\mathrm{C}_{31} \mathrm{H}_{37} \mathrm{~N}_{3} \mathrm{O}_{2}$ (483.64) requires $\mathrm{C}, 76.98 ; \mathrm{H}$, 7.71\%). ${ }^{1} \mathrm{H}$ NMR ( $\delta$, ppm, $\left.\mathrm{CDCl}_{3}\right): 0.74\left(\mathrm{~s}, 3 \mathrm{H}, 18-\mathrm{H}_{3}\right), 0.87$ and $0.97(2 \mathrm{x} \mathrm{s}, 2$ x $2 \mathrm{H}, 2$ "- and 3"$\left.\mathrm{H}_{2}\right), 2.85\left(\mathrm{~m}, 2 \mathrm{H}, 6-\mathrm{H}_{2}\right), 3.63(\mathrm{~d}, 1 \mathrm{H}, J=5.0 \mathrm{~Hz}, 17-\mathrm{H}), 4.26(\mathrm{dd}, 1 \mathrm{H}, J=13.5 \mathrm{~Hz}, J=5.5 \mathrm{~Hz}$, 16a- $\left.\mathrm{H}_{2}\right), 4.60$ (t, 1H, $\left.J=13.5 \mathrm{~Hz}, 16 \mathrm{a}-\mathrm{H}_{2}\right), 5.03\left(\mathrm{~s}, 2 \mathrm{H}, \mathrm{Bn}-\mathrm{H}_{2}\right), 6.72(\mathrm{~d}, 1 \mathrm{H}, J=2.0 \mathrm{~Hz}, 4-\mathrm{H})$, $6.78(\mathrm{dd}, 1 \mathrm{H}, J=8.5 \mathrm{~Hz}, J=2.5 \mathrm{~Hz}, 2-\mathrm{H}), 7.22(\mathrm{~d}, 1 \mathrm{H}, J=8.5 \mathrm{~Hz}, 1-\mathrm{H}), 7.32$ (t, $1 \mathrm{H}, J=7.5 \mathrm{~Hz}$, $\left.4^{\prime}-\mathrm{H}\right), 7.38$ (t, $3 \mathrm{H}, J=7.5 \mathrm{~Hz}, 3^{\prime}$ ' and 5'-H, triazol-H), 7.43 (d, $2 \mathrm{H}, J=7.5 \mathrm{~Hz}, 2^{\prime}-$ and 6'-H). ${ }^{13} \mathrm{C}$ NMR ( $\delta$, ppm, $\mathrm{CDCl}_{3}$ ): 6.5 (C-1"), 7.9 (2C, C-2" and -3"), 17.1 (C-18), 26.0, 27.9, 28.9, 29.8, 31.2, 38.9, 42.3, 43.5, 46.3 (C-16a), 47.0 (C-16), 50.7 (C-13), 69.9 (Bn- $\left.\mathrm{CH}_{2}\right), 78.7$ (C-17), 112.2 (C-2), 114.8 (C-4), 120.8 (triazol-CH)), 126.3 (C-1), 127.4 (C-2' and -6'), 127.4 (C-4'), 128.5 (C-3' and -5'), 132.5 (C-10), 137.2 (C-1'), 137.9 (C-5), 149.6 (triazol-C), 156.7 (C-3).

2.3.44. 3-Benzyloxy-16a-(4'-cyclopentyl-1'H-1',2',3'-triazol-1'-yl)methylestra-1,3,5(10)-trien$17 a-o l(28 b)$

Compound 20 (420.0 mg, $1 \mathrm{mmol}$ ) and cyclopentylacetylene ( $2 \mathrm{mmol}, 0.22 \mathrm{ml})$ were used for the synthesis as described in Section 2.3. The crude product was chromatographed on silica gel with ethyl acetate $/ \mathrm{CH}_{2} \mathrm{Cl}_{2}(2.5: 97.5 \mathrm{v} / \mathrm{v})$ to yield pure $28 \mathrm{~b}(417 \mathrm{mg}, 82 \%)$ as white crystals. $\mathrm{Mp}$ : $197-199{ }^{\circ} \mathrm{C} ; R_{\mathrm{f}}=0.42$ (ss B). (Found: $\mathrm{C}, 77.62 ; \mathrm{H}, 7.85 . \mathrm{C}_{33} \mathrm{H}_{41} \mathrm{~N}_{3} \mathrm{O}_{2}(511.70$ ) requires $\mathrm{C}, 77.46$; $\mathrm{H}, 8.08 \%) .{ }^{1} \mathrm{H}$ NMR ( $\left.\delta, \mathrm{ppm}, \mathrm{CDCl}_{3}\right): 0.76$ (s, 3H, 18- $\left.\mathrm{H}_{3}\right), 2.85$ (m, 2H, 6- $\left.\mathrm{H}_{2}\right), 3.20$ (s, 1H, 1"H), $3.66(\mathrm{~d}, 1 \mathrm{H}, J=5.0 \mathrm{~Hz}, 17-\mathrm{H}), 4.29\left(\mathrm{dd}, 1 \mathrm{H}, J=13.5 \mathrm{~Hz}, J=5.5 \mathrm{~Hz}, 16 \mathrm{a}-\mathrm{H}_{2}\right), 4.62(\mathrm{dd}, 1 \mathrm{H}$, $\left.J=13.5 \mathrm{~Hz}, J=9.5 \mathrm{~Hz}, 16 \mathrm{a}-\mathrm{H}_{2}\right), 5.04\left(\mathrm{~s}, 2 \mathrm{H}, \mathrm{Bn}-\mathrm{H}_{2}\right), 6.72(\mathrm{~s}, 1 \mathrm{H}, 4-\mathrm{H}), 6.78(\mathrm{dd}, 1 \mathrm{H}, J=8.5 \mathrm{~Hz}$, 
$J=2.5 \mathrm{~Hz}, 2-\mathrm{H}), 7.21(\mathrm{~d}, 1 \mathrm{H}, J=8.5 \mathrm{~Hz}, 1-\mathrm{H}), 7.31\left(\mathrm{t}, 1 \mathrm{H}, J=7.0 \mathrm{~Hz}, 4^{\prime}-\mathrm{H}\right), 7.37$ (t, 2H, $J=7.0$ $\mathrm{Hz}, 3^{\prime}$ - and $\left.5^{\prime}-\mathrm{H}\right), 7.43\left(\mathrm{~d}, 2 \mathrm{H}, J=7.0 \mathrm{~Hz}, 2^{\prime}-\right.$ and $\left.6^{\prime}-\mathrm{H}\right) .{ }^{13} \mathrm{C}$ NMR $\left(\delta, \mathrm{ppm}, \mathrm{CDCl}_{3}\right): 17.3(\mathrm{C}-$ 18), 25.2 (2C), 26.1, 28.0, 29.1, 29.8 (2C), 31.3, 33.2, 36.8 (C-1"), 39.0, 42.4, 43.6, 46.4 (C-16a),

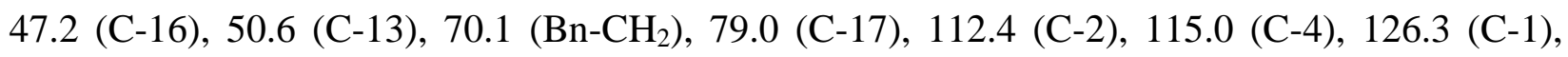
127.4 (C-2' and -6'), 127.8 (C-4'), 128.5 (C-3' and -5'), 133.0 (C-10), 137.5 (C-1'), 137.9 (C-5), $156.9(\mathrm{C}-3)$.

2.3.45. 3-Benzyloxy-16a-(4-cyclohexyl-1H-1,2,3-triazol-1-yl)methyl-estra-1,3,5(10)-trien-17a-ol (28c)

Compound 20 (420.0 mg, $1 \mathrm{mmol}$ ) and cyclohexylacetylene $(2 \mathrm{mmol}, 0.22 \mathrm{ml})$ were used for the synthesis as described in Section 2.3. The crude product was chromatographed on silica gel with ethyl acetate/ $\mathrm{CH}_{2} \mathrm{Cl}_{2}(2.5: 97.5 \mathrm{v} / \mathrm{v})$ to yield pure $28 \mathrm{c}$ (200 $\left.\mathrm{mg}, 76 \%\right)$ as a white solid. $\mathrm{Mp}$ : 223-225 ${ }^{\circ} \mathrm{C} ; R_{\mathrm{f}}=0.44$ (ss B). (Found C, 77.82; H, 8.35. $\mathrm{C}_{34} \mathrm{H}_{43} \mathrm{~N}_{3} \mathrm{O}_{2}$ (525.72) requires C, 77.68; $\mathrm{H}, 8.24 \%) .{ }^{1} \mathrm{H}$ NMR $\left(\delta, \mathrm{ppm}, \mathrm{CDCl}_{3}\right): 0.75$ (s, 3H, 18- $\left.\mathrm{H}_{3}\right), 2.84$ (m, 3H, 6-H2, 1"- $\left.\mathrm{H}\right), 3.64(\mathrm{~s}, 1 \mathrm{H}$, $17-\mathrm{H}), 4.37\left(\mathrm{~m}, 1 \mathrm{H}, 16 \mathrm{a}-\mathrm{H}_{2}\right), 4.69\left(\mathrm{~m}, 1 \mathrm{H}, 16 \mathrm{a}-\mathrm{H}_{2}\right), 5.03\left(\mathrm{~s}, 2 \mathrm{H}, \mathrm{Bn}-\mathrm{H}_{2}\right), 6.72(\mathrm{~d}, 1 \mathrm{H}, J=1.5 \mathrm{~Hz}$, 4-H), $6.78(\mathrm{dd}, 1 \mathrm{H}, J=8.5 \mathrm{~Hz}, J=2.5 \mathrm{~Hz}, 2-\mathrm{H}), 7.22(\mathrm{~d}, 1 \mathrm{H}, J=8.5 \mathrm{~Hz}, 1-\mathrm{H}), 7.32(\mathrm{t}, 1 \mathrm{H}, J=$ $\left.7.0 \mathrm{~Hz}, 4^{\prime}-\mathrm{H}\right), 7.38$ (t, $2 \mathrm{H}, J=7.0 \mathrm{~Hz}, 3^{\prime}-$ and 5'-H), 7.43 (d, 2H, $J=7.0 \mathrm{~Hz}, 2^{\prime}-$ and 6' $-\mathrm{H}$

2.3.46. 3-Benzyloxy-16a-(4-phenyl-1H-1,2,3-triazol-1-yl)methyl-estra-1,3,5(10)-trien-17a-ol (28d)

Compound $20(420.0 \mathrm{mg}, 1 \mathrm{mmol})$ and phenylacetylene $(2 \mathrm{mmol}, 0.22 \mathrm{ml})$ were used for the synthesis as described in Section 2.3. The crude product was chromatographed on silica gel with ethyl acetate $/ \mathrm{CH}_{2} \mathrm{Cl}_{2}(5: 95 \mathrm{v} / \mathrm{v})$ to yield pure $\mathbf{2 8 d}$ (337 mg, 64\%) as a white solid. Mp: 205-206 ${ }^{\circ} \mathrm{C} ; R_{\mathrm{f}}=0.46$ (ss B). (Found C, 78.42; H, 7.32. $\mathrm{C}_{34} \mathrm{H}_{37} \mathrm{~N}_{3} \mathrm{O}_{2}$ (519.68) requires $\mathrm{C}, 78.58 ; \mathrm{H}, 7.18 \%$ ). ${ }^{1} \mathrm{H}$ NMR ( $\delta$, ppm, $\left.\mathrm{CDCl}_{3}\right): 0.76\left(\mathrm{~s}, 3 \mathrm{H}, 18-\mathrm{H}_{3}\right), 2.87\left(\mathrm{~m}, 2 \mathrm{H}, 6-\mathrm{H}_{2}\right), 3.68(\mathrm{~d}, 1 \mathrm{H}, J=5.0 \mathrm{~Hz}, 17-$ $\mathrm{H}), 4.41\left(\mathrm{dd}, 1 \mathrm{H}, J=13.5 \mathrm{~Hz}, J=5.5 \mathrm{~Hz}, 16 \mathrm{a}-\mathrm{H}_{2}\right), 4.69\left(\mathrm{t}, 1 \mathrm{H}, J=13.5 \mathrm{~Hz}, 16 \mathrm{a}-\mathrm{H}_{2}\right), 5.04(\mathrm{~s}, 2 \mathrm{H}$, Bn- $\mathrm{H}_{2}$ ), 6.73 (s, 1H, 4-H), 6.79 (dd, 1H, $\left.J=8.0 \mathrm{~Hz}, J=2.0 \mathrm{~Hz}, 2-\mathrm{H}\right), 7.22(\mathrm{~d}, 1 \mathrm{H}, J=8.0 \mathrm{~Hz}, 1-$ H), 7.38 (m, 8H, 2'-, 3'-, 4'-, 5'- and 6'-H, 3"-, 4"'- and 5'-H), 7.84 (d, 2H, $J=7.5 \mathrm{~Hz}, 2$ '- and 6"-H), 7.89 (s, $1 \mathrm{H}$, triazol-H). ${ }^{13} \mathrm{C}$ NMR $\left(\delta, \mathrm{ppm}, \mathrm{CDCl}_{3}\right)$ : 17.1 (C-18), 26.0, 27.9, 29.8, 31.2, 38.9, 42.2, 43.5, 46.4 (C-13), 47.0 (C-16), 50.8 (C-16a), 69.9 (Bn- $\left.\mathrm{CH}_{2}\right), 78.8$ (C-17), 112.3 (C-2), 114.8 (C-4), 120.7 (triazol-CH), 125.7 (C-2' and -6'), 126.3 (C-1), 127.4 (C-2" and -6”), 127.8 
(C-4'), 128.3 (C-4"), 128.5 (C-3" and -5”), 128.9 (C-3' and -5'), 130.2 (C-10), 132.8 (C-1'), 137.3 (C-1”), 137.9 (C-5), 147.1 (triazol-C), 156.7 (C-3).

2.3.47. 3-Benzyloxy-16a-[4'-(4''-nitro-benzoyloxymethyl)-1'H-1',2',3'-triazol-1'-yl]methylestra1,3,5(10)-trien-17a-ol (28e)

Compound 20 (420 mg, $1 \mathrm{mmol}$ ) and propargyl 4-nitrobenzoate ( $2 \mathrm{mmol}, 210 \mathrm{mg}$ ) were used for the synthesis as described in Section 2.3. The crude product was chromatographed on silica gel with ethyl acetate $/ \mathrm{CH}_{2} \mathrm{Cl}_{2}(5: 95 \mathrm{v} / \mathrm{v})$ to yield pure $28 \mathrm{e}(610 \mathrm{mg}, 98 \%)$ as a yellow solid. Mp: $75-77{ }^{\circ} \mathrm{C} ; R_{\mathrm{f}}=0.45$ (ss B). (Found C, 69.57; H, 61.32. $\mathrm{C}_{36} \mathrm{H}_{38} \mathrm{~N}_{4} \mathrm{O}_{6}(622.71)$ requires $\mathrm{C}, 69.44 ; \mathrm{H}$, 6.15\%). ${ }^{1} \mathrm{H}$ NMR ( $\delta$, ppm, DMSO-d $\left.\mathrm{d}_{6}\right): 0.66$ (s, 3H, 18- $\left.\mathrm{H}_{3}\right), 2.71$ (m, 2H, 6- $\left.\mathrm{H}_{2}\right), 3.57$ (s, 1H, 16H), $4.29\left(\mathrm{dd}, 1 \mathrm{H}, J=13.5 \mathrm{~Hz}, J=8.5 \mathrm{~Hz}, 16 \mathrm{a}-\mathrm{H}_{2}\right), 4.47(\mathrm{dd}, 1 \mathrm{H}, J=13.5 \mathrm{~Hz}, J=8.5 \mathrm{~Hz}, 16 \mathrm{a}-$ $\left.\mathrm{H}_{2}\right), 4.85(\mathrm{~d}, 1 \mathrm{H}, J=5.0 \mathrm{~Hz}, 17-\mathrm{H}), 5.44\left(\mathrm{~s}, 2 \mathrm{H}, \mathrm{Bn}-\mathrm{H}_{2}\right), 6.65(\mathrm{~s}, 1 \mathrm{H}, 4-\mathrm{H}), 6.72(\mathrm{~d}, 1 \mathrm{H}, J=8.5$ Hz, 2-H), 7.14 (d, 1H, J = 8.5 Hz, 1-H), 7.29 (t, 1H, $\left.J=7.5 \mathrm{~Hz}, 4^{\prime}-\mathrm{H}\right), 7.35$ (t, 2H, $J=7.5 \mathrm{~Hz}, 3^{\prime}-$ and 5'-H), $7.40\left(\mathrm{~d}, 2 \mathrm{H}, J=7.5 \mathrm{~Hz}, 2^{\prime}-\right.$ and 6'-H), 8.17 (d, 2H, $J=8.5 \mathrm{~Hz}, 3$ '- and 5'-H), 8.28 (s, $1 \mathrm{H}$, triazol H), $8.31(\mathrm{~d}, 2 \mathrm{H}, J=8.5 \mathrm{~Hz}, 2$ "- and $6 "-\mathrm{H}) .{ }^{13} \mathrm{C}$ NMR $\left(\delta, \mathrm{ppm}, \mathrm{DMSO}-\mathrm{d}_{6}\right): 16.9(\mathrm{C}-$ 18), 25.6, 27.5, 28.4, 29.2, 31.1, 38.5, 39.8, 39.9, 43.2, 45.9 (C-16a), 46.2 (C-16), 53.4 (C-13), 58.7 (linker $\mathrm{CH}_{2}$ ), 68.9 (Bn-CH 2$), 78.0$ (C-17), 112.1 (C-2), 114.4 (C-4), 123.8 (C-2" and -6"), 125.0 (triazol CH), 126.1 (C-1), 127.4 (C-2' and -6'), 127.5 (C-4'), 128.3 (C-3' and -5'), 130.6 (C-3" and -5”), 132.3 (C-10), 134.7 (C-1'), 137.3 (C-5), 141.0 (C-1"), 150.2 (triazol C), 156.0 (C-3), $163.9(\mathrm{C}=\mathrm{O})$.

2.3.48. 3-Benzyloxy-16a-(4'-hydroxymethyl-1'H-1',2',3'-triazol-1'-yl)methylestra-1,3,5(10)trien-17 $\alpha$-ol (28f)

Compound 28e (220 mg, $0.5 \mathrm{mmol})$ was dissolved in methanol (10 ml) containing $\mathrm{NaOCH}_{3}(14$ $\mathrm{mg}, 0.25 \mathrm{mmol}$ ), and the solution was allowed to stand for $24 \mathrm{~h}$. It was then diluted with water, and the precipitate separating out was filtered off and recrystallized from methanol to afford $\mathbf{2 8 f}$ $(126 \mathrm{mg}, 53 \%)$ as a white crystalline product. Mp: $86-88{ }^{\circ} \mathrm{C} ; R_{\mathrm{f}}=0.25$ (ss B). (Found C, 73.68; $\mathrm{H}, 7.63 . \mathrm{C}_{29} \mathrm{H}_{35} \mathrm{~N}_{3} \mathrm{O}_{3}$ (473.61) requires C, 73.54; H, 7.45\%). ${ }^{1} \mathrm{H}$ NMR ( $\delta$, ppm, DMSO-d $\mathrm{d}_{6}$ ): 0.68 (s, 3H, 18- $\left.\mathrm{H}_{3}\right), 2.74\left(\mathrm{~m}, 2 \mathrm{H}, 6-\mathrm{H}_{2}\right), 3.58(\mathrm{brs}, 1 \mathrm{H}, \mathrm{OH}), 4.26\left(\mathrm{t}, 1 \mathrm{H}, J=8.5 \mathrm{~Hz}, 16 \mathrm{a}-\mathrm{H}_{2}\right), 4.43(\mathrm{dd}$, $\left.1 \mathrm{H}, J=13.0 \mathrm{~Hz}, J=7.0 \mathrm{~Hz}, 16 \mathrm{a}-\mathrm{H}_{2}\right), 4.51\left(\mathrm{~d}, 2 \mathrm{H}, J=5.0 \mathrm{~Hz}\right.$, linker $\left.\mathrm{H}_{2}\right), 4.85(\mathrm{~d}, 1 \mathrm{H}, J=4.0 \mathrm{~Hz}$, 17-H), 5.04 (s, 2H, Bn- $\left.\mathrm{H}_{2}\right), 5.13$ (brs, 1H, OH), 6.68 (s, 1H, 4-H), 6.74 (d, 1H, J = 8.5 Hz, 2-H), 


\subsection{Determination of the antiproliferative activities}

The growth-inhibitory effects of the compounds were tested in vitro by means of the MTT assay against a gynecological panel containing two breast cancer cell lines (MCF-7, MD-MB231) and two cell lines isolated from cervical malignancies (HeLa, SiHa) [11]. All cell lines were obtained from the European Collection of Cell Cultures (Salisbury, UK). The cells were maintained in minimal essential medium supplemented with $10 \%$ fetal bovine serum (FBS), $1 \%$ non-essential amino acids and an antibiotic-antimycotic mixture (AAM). All chemicals, if otherwise not specified, were purchased from Sigma-Aldrich Ltd. (Budapest, Hungary). All cell lines were grown in a humidified atmosphere of $5 \% \mathrm{CO}_{2}$ at $37{ }^{\circ} \mathrm{C}$. For pharmacological investigations, $10 \mathrm{mM}$ stock solutions of the tested compounds were prepared with dimethyl sulfoxide (DMSO). The highest applied DMSO concentration of the medium (0.3\%) did not have any substantial effect on the determined cellular functions. Cells were seeded into 96-well plates (5000 cells/well), allowed to stand overnight under cell culturing conditions, and the medium containing the tested compounds at two final concentrations $(10$ or $30 \mu \mathrm{M})$ was then added. After a 72-hour incubation viability was determined by the addition of $20 \mu 1$ 3-(4,5-dimethylthiazol-2yl)-2,5-diphenyltetrazolium bromide (MTT) solution $(5 \mathrm{mg} / \mathrm{ml})$. The formazan crystals precipitated in $4 \mathrm{~h}$ were solubilized in DMSO and the absorbance was determined at $545 \mathrm{~nm}$ with an ELISA plate reader utilizing untreated cells as controls. The most effective compounds eliciting at least $60 \%$ growth inhibition at $10 \mu \mathrm{M}$ were tested again with a set of dilutions (0.3-30 $\mu \mathrm{M}$ ) in order to determine the $\mathrm{IC}_{50}$ values by means of Graphpad Prism 4.0 (Graphpad Software; San Diego, CA, US). These promising compounds were additionally tested using nonmalignant murine fibroblasts (NIH-3T3) to obtain preliminary data concerning cancer selectivity of the tested molecules. Two independent experiments were performed with 5 parallel wells and 
cisplatin (Ebewe GmbH, Unterach, Austria), an agent administered clinically in the treatment of certain gynecological malignancies, was used as reference compound.

\section{Results and discussion}

\subsection{Synthetic studies}

To prepare novel steroid triazoles via 1,3-dipolar cycloaddition, we chose the 3-methoxy- and 3benzyloxy-16-hydroxymethylestra-1,3,5(10)-trien-17-ol diastereomers (5-8 and 9-12). The synthesis strategy for the preparation of the starting diols (21-28) is illustrated in Scheme 1. The synthesis of steroidal 1,2,3-triazoles by CuAAC is outlined in Scheme 2.

Stereoselective tosylation of 5-8 and bromination of 9-12 gave 5b-8b and 9c-12c, respectively, which then underwent facile $\mathrm{S}_{\mathrm{N}} 2$ substitution with $\mathrm{NaN}_{3}$ in $N, N$-dimethylformamide to furnish the corresponding 16-azidomethyl compounds (13-16 and 17-20).

The 16-azido compounds were subjected to the azide-alkyne CuAAC reaction with different alkyl- and aryl-acetylenes. The azide-alkyne reactions of these compounds were carried out with $\mathrm{CuI}$ as catalyst in the presence of $\mathrm{Et}_{3} \mathrm{~N}$ in $\mathrm{CH}_{2} \mathrm{Cl}_{2}$ under reflux conditions to obtain the required 3methoxy- and 3-benzyloxyestra-1,3,5(10)-trien-16-(1',4'-substituted 1',2',3')-triazolyl derivatives (21-24 and 25-28).

\subsection{Determination of the antiproliferative properties of the 16-triazolylmethyl diastereomers}

We have published recently that introduction of a substituted triazole moiety onto different positions of the estrane skeleton might increase the antiproliferative properties of estrone derivatives [12]. It was also established that the presence of certain alkyl or aralkyl protecting groups at the phenolic $\mathrm{OH}$ function is advantageous. Concerning that 16hydroxymethylene-17-hydroxy derivatives of estrone-3-methyl ether or 3-benzyl ether (5a-12a) displayed substantial cytostatic potential against different types of breast cancer cell lines, these compounds might be suitable for directed modifications with the aim of developing potentially more active antiproliferative steroidal derivatives [13]. In the light of the above-mentioned recent observations, here we aimed to combine the substituted triazole and the 16,17-disubstituted estrone 3-ether moieties. The present study included an evaluation of the direct antiproliferative 

of MTT assays against human adherent cervical (SiHa, HeLa) and breast cancer (MCF-7 and MDA-MB-231) cell lines.

The antiproliferative activities of the newly synthesized heterocyclic compounds depended on the nature of the protecting group at the 3-hydroxy function and on the orientation of the substituents at C-16 and C-17. In general, the 3-methyl ethers (21-24) exhibited weak antiproliferative action; none of them exerted any substantial effect at $10 \mu \mathrm{M}$ (Table 1). All diastereomers of the 3-benzyl ether series (25-28) proved to be more potent in comparison with their 3-methyl ether counterparts (Table 2). This is in agreement with our earlier results [14]. Based on the substantial difference of the two groups, i.e. that of 3-methyl ethers and 3-benzyl ethers, it can be concluded that only the latter derivatives are promising from pharmacological point of view.

Concerning the orientation of the substituents at position C-16 and C-17, the $16 \beta, 17 \beta$ derivatives (25a-f) displayed outstanding growth-inhibitory properties. Two derivatives bearing similar cycloalkyl groups at position C-4' displayed substantial selective antiproliferative action against the triple-negative breast cancer cell line MDA-MB-231 with $\mathrm{IC}_{50}$ values in the low micromolar range. It should be underlined that $\mathbf{2 5 b}$ and 25c did not significantly influence the proliferation of other cell lines tested, including the non-cancerous fibroblast. Although both the 4'-cyclohexyl (25c) and the 4'-phenyl derivative (25d) have six-membered substituents, their cytostatic behavior is completely different. This might be attributed to the different steric structure of the two rings (chair or planar) at C-4'. Compound 25d exerted potent

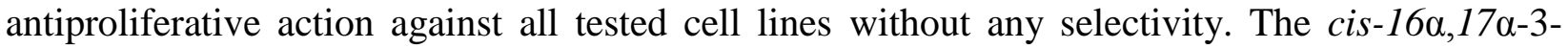
benzyl ethers (28a-f) were less potent than their $\beta, \beta$-counterparts $(\mathbf{2 5 a} \mathbf{a}-\mathbf{f})$, except for $\mathbf{2 8 d}$, which

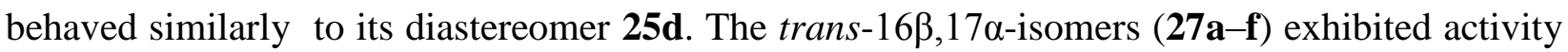
exclusively on the breast cancer cell lines. Surprisingly, the tendency observed earlier (in the case of compounds 25a-f) concerning the nature of C-4' substituent was not valid here. Only 26a and 26e inhibited cell growth markedly, but with no tumor selectivity. It's worth mentioning that trans-16 $\alpha, 17 \beta$ isomer 26c was the sole compound, which inhibited the proliferation of HPV 16+ squamous cell carcinoma $\mathrm{SiHa}$, showing an $\mathrm{IC}_{50}$ value comparable with that of cisplatin. 
In view of the cell lines, it should be noted that triple-negative breast cancer cell line MDA-MB231 proved to be the most sensitive and all calculated $\mathrm{IC}_{50}$ values were lower than that of the reference agent cisplatin $(19.1 \mu \mathrm{M})$.

Regarding the present and earlier results obtained for 16,17-disubstituted 3-benzyl ethers, it can be stated that introduction of a substituted triazolyl moiety onto the C-16 methylene group of the cis isomers proved to be advantageous. In the case of compounds $\mathbf{2 5} \mathbf{b}$ and $\mathbf{2 5 c}$, both the antiproliferative potential and the tumor selectivity were markedly improved.

\section{Acknowledgement}

The work of Anita Kiss was supported by a PhD Fellowship of the Talentum Fund of Richter Gedeon Plc. (Budapest). Financial support from the Economic Development and Innovation Operative Programme of Hungary (GINOP-2.3.2-15-2016-00038) and Ultrafast physical processes in atoms, molecules, nanostructures and biological systems (No: EFOP-3.6.2.2017-00005) is gratefully acknowledged. This research was supported by the Hungarian Scientific Research Fund (OTKA K113150). Ministry of Human Capacities, Hungary grant 20391-3/2018/FEKUSTRAT is acknowledged. 


\section{References}

[1] Tietze LF, Bell HP, Chandrasekhar S, Natural product hybrids as new leads drug discovery. Angew Chem Int Ed 2003;42:3996-4028.

[2] Mehta G, Singh V, Hybrid system through natural product leads: An approach towards new molecular entities. Chem Soc Rev 2002;31:324-334.

[3] Adamec J, Beckert R, Weiß D, Klimešová V, Waisser K, Möllmann U, Kaustová J, Buchta V. Hybride molecules of estrone: New Compound with potential antibacterial, antifungal and antiproliferative activities. Bioorg Med Chem 2007;15:2898-2906.

[4] Kuduk, SD, Zheng FF, Sepp-Lorenzino L, Rosen N, Danishefsy SJ. Synthesis and Evaluation of geldanamycin-estradiol hybrids. Bioorg Med Chem 1999;9:1233-1238.

[5] Gupta A, Saha P, Descôteaux C, Leblanc V, Asselin É, Bérubé G. Design, synthesis and biological evaluation of estradiol-chlorambucil hybrids as anticancer agents. Bioorg Med Chem Lett 2010;20:1614-1618.

[6] Schneider Gy, Vass A, Vincze I, Sohár P. Neighbouring Group Partcipation in the 16Hydroxymethyl-3-methoxyestre-1,3,5(10)-trien-17ß-ol Series. Liebigs Ann Chem 1988;267-273.

[7] Schneider Gy, Hackler L, Sohár P. Preparation of 16 $\alpha$-Hydroxymethyl-3-methoxyestra1,3,5(10)-trien 17 $\alpha$-ol and Solvolysis Investigation. Liebigs Ann Chem 1988;679-683.

[8] Tapolcsányi P, Wölfling J, Falkay G, Márki Á, Minorics R, Schneider Gy. Synthesis and receptor-binding examination of 16-hydroxymethyl-3,17-estradiol stereoisomers. Steroids 2002;67:671-678.

[9] Meldal M, Tornøe CW. Cu-Catalyzed azide-alkyne cycloaddition. Chem Rev.

2008;108:2952-3015.

[10] Szájli Á, Wölfling J, Mernyák E, Minorics R, Márki Á, Falkay G, Schneider Gy. Neighbouring group participation Part 16. Stereoselective synthesisi and receptor-binding examination of the four stereoisomers of 16-bromomethyl-3,17-estradiols. Steroids 2006; $71: 141-153$.

[11] Mosmann T. Rapid colorimetrc assay for cellural growth and survival: application to prolyferation and cytotoxicity assay. J immunol Methods 1983;65:55-63. 
[12] Mernyák E, Kovács I, Minorics R, Sere P, Czégány Sinka I, Wölfling, Schneider G, Újfaludi Zs, Boros I, Ocsovszki I, Varjj ga M, Zupkó I. Synthesis of trans-16-triazolyl-13amethyl-17-estradiol diastereomers and the effects of structural modifications on their in vitro antiproliferative activities. J Steroid Biochem Mol Biol 2015;150:123-134.

[13] Sinka I, Kiss A, Mernyák E, Wölfling E, Schneider G. Ocsovszki I, Kuo CY, Wang HC, Zupkó I. Antiproliferative and antimetastatic properties of 3-benzyloxy-16-hydroxymethyleneestardiol analogs agains breast cancer cell lines. Eur J Pharm Sciences 2018;123:362-370.

[14] Frank E, Molnar J, Zupko I, Kadar Z, Wölfling J. Synthesis of novel steroidal 17 $\alpha$-triazolyl derivatives via $\mathrm{Cu}(\mathrm{I})$-catalyzed azide-alkyne cycloaddition, and an evaluation of their cytotoxic activity in vitro. Steroids 2011;76:1141-1148. 


\section{Legends for Schemes and Tables}

Scheme 1 Reagents and conditions: (i) NaOMe, HCOOEt, anhydrous toluene, $50{ }^{\circ} \mathrm{C}$; (ii) $\mathrm{KBH}_{4}$, $\mathrm{MeOH}$; (iii) $\mathrm{KOAc}, \mathrm{CH}_{3} \mathrm{COOH}, \mathrm{NaOMe} / \mathrm{MeOH}$.

Scheme 2 Reagents and conditions: (i) appropriate alkyne, TEA, $\mathrm{CuI}, \mathrm{CH}_{2} \mathrm{Cl}_{2}, 40{ }^{\circ} \mathrm{C}, 24 \mathrm{~h}$; (ii) $\mathrm{NaOMe}, \mathrm{MeOH}, 24 \mathrm{~h}$.

Table 1 Antiproliferative activities of compounds $21 \mathbf{a}-\mathbf{f}, \mathbf{2 2} \mathbf{a}-\mathbf{f}, \mathbf{2 3 a}-\mathbf{f}$ and $24 \mathbf{a}-\mathbf{f}$

Table 2 Antiproliferative activities of compounds $25 \mathbf{a}-\mathbf{f}, \mathbf{2 6} \mathbf{a}-\mathbf{f}, \mathbf{2 7} \mathbf{a}-\mathbf{f}$ and $28 \mathbf{a}-\mathbf{f}$ 
Table 1

\begin{tabular}{|c|c|c|c|c|c|}
\hline & & $\begin{array}{l}\text { Grov } \\
{[\mathrm{c}}\end{array}$ & $\begin{array}{l}\text { Inhibition, } \% \\
\text { ulated } \mathrm{IC}_{50}(\mu\end{array}$ & & \\
\hline & $\begin{array}{r}\text { Conc. } \\
(\mu \mathrm{M})\end{array}$ & HeLa & $\mathrm{SiHa}$ & MCF-7 & $\begin{array}{c}\text { MDA-MB- } \\
231\end{array}$ \\
\hline 21 & & & & & \\
\hline & 10 & $<20$ & $21.28 \pm 1.88$ & $<20$ & $<20$ \\
\hline $\mathbf{a}$ & 30 & $<20$ & $28.71 \pm 2.20$ & $46.42 \pm 1.47$ & $<20$ \\
\hline & 10 & $<20$ & $<20$ & $<20$ & $<20$ \\
\hline b & 30 & $39.86 \pm .38$ & $<20$ & $57.42 \pm 1.77$ & $29.88 \pm 1.57$ \\
\hline & 10 & $<20$ & $<20$ & $<20$ & $<20$ \\
\hline c & 30 & $40.22 \pm 1.02$ & $<20$ & $70.84 \pm 1.55$ & $37.96 \pm 1.55$ \\
\hline & 10 & $<20$ & $<20$ & $<20$ & $<20$ \\
\hline d & 30 & $44.16 \pm 0.48$ & $<20$ & $54.93 \pm 1.78$ & $38.28 \pm 1.84$ \\
\hline & 10 & $<20$ & $23.91 \pm 1.61$ & $34.23 \pm 3.10$ & $<20$ \\
\hline $\mathrm{e}$ & 30 & $37.18 \pm 1.65$ & $54.72 \pm 0.48$ & $76.26 \pm 0.72$ & $35.93 \pm 2.13$ \\
\hline$f$ & 10 & $<20$ & $28.06 \pm 1.99$ & $29.45 \pm 1.67$ & $<20$ \\
\hline 1 & 30 & $41.03 \pm 0.77$ & $57.69 \pm 1.12$ & $70.23 \pm 1.35$ & $34.81 \pm 2.88$ \\
\hline 22 & & & & & \\
\hline 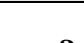 & 10 & $<20$ & $25.55 \pm 1.01$ & $<20$ & $<20$ \\
\hline a & 30 & $<20$ & $34.78 \pm 2.47$ & $57.43 \pm 1.91$ & $<20$ \\
\hline b & 10 & $<20$ & $<20$ & $<20$ & $<20$ \\
\hline 10 & 30 & $<20$ & $26.57 \pm 2.26$ & $67.59 \pm 1.65$ & $<20$ \\
\hline 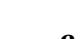 & 10 & $<20$ & $<20$ & $<20$ & $<20$ \\
\hline $\mathbf{c}$ & 30 & $<20$ & $29.90 \pm 2.59$ & $69.68 \pm 0.77$ & $<20$ \\
\hline d & 10 & $<20$ & $<20$ & $<20$ & $<20$ \\
\hline a & 30 & $<20$ & $29.96 \pm 1.79$ & $70.75 \pm 1.05$ & $14.54 \pm 1.32$ \\
\hline & 10 & $<20$ & $<20$ & $<20$ & $<20$ \\
\hline e & 30 & $<20$ & $38.69 \pm 2.09$ & $63.12 \pm 2.14$ & $<20$ \\
\hline$f$ & 10 & $<20$ & $<20$ & $22.02 \pm 1.61$ & $<20$ \\
\hline $\mathbf{I}$ & 30 & $<20$ & $37.79 \pm 1.04$ & $50.94 \pm 1.55$ & $<20$ \\
\hline 23 & & & & & \\
\hline & 10 & $<20$ & $<20$ & $<20$ & $<20$ \\
\hline $\mathbf{a}$ & 30 & $31.14 \pm 1.28$ & $<20$ & $28.72 \pm 0.93$ & $25.08 \pm 3.15$ \\
\hline h & 10 & $<20$ & $<20$ & $<20$ & $<20$ \\
\hline b & 30 & $58.25 \pm 2.03$ & $<20$ & $48.01 \pm 1.31$ & $<20$ \\
\hline & 10 & $<20$ & $30.97 \pm 2.69$ & $<20$ & $<20$ \\
\hline c & 30 & $<20$ & $33.89 \pm 2.35$ & $<20$ & $<20$ \\
\hline d & 10 & $<20$ & $<20$ & $<20$ & $<20$ \\
\hline d & 30 & $26.90 \pm 2.15$ & $<20$ & $63.27 \pm 0.82$ & $<20$ \\
\hline ? & 10 & $<20$ & $<20$ & $<20$ & $<20$ \\
\hline $\mathrm{e}$ & 30 & $<20$ & $37.53 \pm 3.00$ & $33.94 \pm 0.75$ & $28.19 \pm 0.96$ \\
\hline$f$ & 10 & $<20$ & $29.13 \pm 1.59$ & $<20$ & $<20$ \\
\hline $\mathbf{I}$ & 30 & $26.61 \pm 0.57$ & $43.85 \pm 3.32$ & $38.45 \pm 1.93$ & $43.85 \pm 3.32$ \\
\hline 24 & & & & & \\
\hline 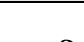 & 10 & $<20$ & $<20$ & $<20$ & $<20$ \\
\hline a & 30 & $89.01 \pm 0.47$ & $<20$ & $78.65 \pm 0.78$ & $46.21 \pm 1.54$ \\
\hline b & 10 & $<20$ & $<20$ & $<20$ & $<20$ \\
\hline D & 30 & $34.18 \pm 0.81$ & $<20$ & $31.07 \pm 2.36$ & $<20$ \\
\hline c & 10 & $<20$ & $<20$ & $<20$ & $<20$ \\
\hline $\mathrm{c}$ & 30 & $49.11 \pm 0.55$ & $<20$ & $43.22 \pm 1.52$ & $<20$ \\
\hline
\end{tabular}




\begin{tabular}{cc|cccc}
\hline \multirow{2}{*}{$\mathbf{d}$} & 10 & $<20$ & $<20$ & $<20$ & $<20$ \\
& 30 & $42.13 \pm 1.66$ & $<20$ & $55.41 \pm 0.76$ & $<20$ \\
\hline \multirow{2}{*}{$\mathbf{e}$} & 10 & $<20$ & $<20$ & $<20$ & $<20$ \\
& 30 & $83.66 \pm 0.34$ & $42.06 \pm 2.50$ & $70.11 \pm 1.06$ & $50.27 \pm 2.00$ \\
\hline \multirow{2}{*}{$\mathbf{f}$} & 10 & $<20$ & $<20$ & $22.34 \pm 2.06$ & $<20$ \\
& 30 & $84.77 \pm 1.18$ & $29.80 \pm 1.66$ & $68.27 \pm 1.19$ & $47.74 \pm 1.21$ \\
\hline \multirow{2}{*}{ cisplatin } & 10 & $42.61 \pm 2.33$ & $86.84 \pm 0.50$ & $53.03 \pm 2.29$ & $20.84 \pm 0.81$ \\
& 30 & $99.93 \pm 0.26$ & $90.18 \pm 1.78$ & $86.90 \pm 1.24$ & $74.47 \pm 1.20$ \\
& & {$[12.43]$} & {$[7.84]$} & {$[5.78]$} & {$[19.13]$}
\end{tabular}


Table 2

\begin{tabular}{|c|c|c|c|c|c|c|}
\hline & & $\begin{array}{r}\text { Grov } \\
{[\mathrm{c}}\end{array}$ & $\begin{array}{l}\text { Inhibition, } \% \\
\text { ulated } \mathrm{IC}_{50}(\mu\end{array}$ & & & \\
\hline & $\begin{array}{l}\text { Conc. } \\
(\mu \mathrm{M})\end{array}$ & $\mathrm{HeLa}$ & $\mathrm{SiHa}$ & MCF-7 & $\begin{array}{c}\text { MDA-MB- } \\
231\end{array}$ & NIH-3T3 \\
\hline 25 & & & & & & \\
\hline & 10 & $44.94 \pm 1.04$ & $21.17 \pm 2.05$ & $41.71 \pm 0.64$ & $47.32 \pm 1.15$ & $44.91 \pm 1.36$ \\
\hline $\mathbf{a}$ & 30 & $52.45 \pm 2.39$ & $66.23 \pm 0.86$ & $64.32 \pm 0.56$ & $71.49 \pm 0.75$ & $91.28 \pm 0.50$ \\
\hline & 10 & $51.49 \pm 3.62$ & $49.36 \pm 1.69$ & $44.58 \pm 1.50$ & $93.00 \pm 0.26$ & $44.81 \pm 1.50$ \\
\hline b & 30 & $62.58 \pm 2.21$ & $73.94 \pm 2.04$ & $50.52 \pm 3.26$ & $\begin{array}{c}93.71 \pm 0.09 \\
{[3.33]}\end{array}$ & $59.09 \pm 0.73$ \\
\hline & & $54.70 \pm 1.88$ & $49.58 \pm 2.11$ & $44.04 \pm 3.32$ & $77.13 \pm 1.07$ & \\
\hline c & $\begin{array}{l}10 \\
30\end{array}$ & $53.66 \pm 2.56$ & $61.83 \pm 2.77$ & $59.33 \pm 2.99$ & $\begin{array}{c}88.81 \pm 0.55 \\
{[5.91]}\end{array}$ & \\
\hline & 10 & $64.14 \pm 0.86$ & $70.88 \pm 1.03$ & $73.41 \pm 1.22$ & $95.04 \pm 0.16$ & $95.60 \pm 0.25$ \\
\hline d & 30 & $\begin{array}{c}90.12 \pm 0.99 \\
{[2.28]}\end{array}$ & $\begin{array}{c}94.14 \pm 0.29 \\
{[4.05]}\end{array}$ & $\begin{array}{c}80.16 \pm 3.40 \\
{[3.91]}\end{array}$ & $\begin{array}{c}95.60 \pm 0.06 \\
{[3.65]}\end{array}$ & $\begin{array}{c}98.22 \pm 0.04 \\
{[3.34]}\end{array}$ \\
\hline & 10 & $<20$ & $<20$ & $41.63 \pm 2.83$ & $21.96 \pm 0.73$ & \\
\hline e & 30 & $92.12 \pm 0.25$ & $89.25 \pm 0.68$ & $97.00 \pm 0.11$ & $95.22 \pm 0.91$ & \\
\hline & 10 & $45.08 \pm 0.72$ & $41.26 \pm 1.25$ & $55.41 \pm 1.26$ & $55.57 \pm 1.50$ & \\
\hline $\mathbf{f}$ & 30 & $39.39 \pm 0.49$ & $52.60 \pm 1.31$ & $62.52 \pm 0.67$ & $88.92 \pm 0.99$ & \\
\hline 26 & & & & & & \\
\hline & 10 & $37.98 \pm 2.68$ & $<20$ & $72.42 \pm 2.19$ & $46.43 \pm 2.05$ & $85.50 \pm 1.22$ \\
\hline $\mathbf{a}$ & 30 & $96.56 \pm 0.11$ & $96.71 \pm 0.17$ & $\begin{array}{c}98.72 \pm 0.09 \\
{[6.11]}\end{array}$ & $97.96 \pm 0.17$ & $\begin{array}{c}97.63 \pm 0.12 \\
{[5.97]}\end{array}$ \\
\hline b & 10 & $38.55 \pm 1.32$ & $<20$ & $31.80 \pm 1.35$ & $17.13 \pm 2.36$ & \\
\hline b & 30 & $43.97 \pm 2.23$ & $<20$ & $84.44 \pm 0.71$ & $37.72 \pm 2.28$ & \\
\hline & 10 & $36.30 \pm 1.45$ & $<20$ & $24.95 \pm 2.15$ & $<20$ & \\
\hline c & 30 & $35.53 \pm 1.24$ & $<20$ & $74.73 \pm 1.00$ & $<20$ & \\
\hline d & 10 & $<20$ & $<20$ & $47.25 \pm 1.78$ & $45.55 \pm 2.63$ & \\
\hline d & 30 & $22.15 \pm 1.29$ & $<20$ & $57.30 \pm 0.77$ & $59.79 \pm 1.22$ & \\
\hline & 10 & $<20$ & $<20$ & $68.51 \pm 0.71$ & $89.24 \pm 0.70$ & $31.41 \pm 2.21$ \\
\hline $\mathbf{e}$ & 30 & $96.98 \pm 0.33$ & $96.91 \pm 0.14$ & $\begin{array}{c}99.12 \pm 0.07 \\
{[6.53]}\end{array}$ & $\begin{array}{c}97.73 \pm 0.23 \\
{[5.69]}\end{array}$ & $\begin{array}{c}99.01 \pm 0.05 \\
{[11.75]}\end{array}$ \\
\hline & 10 & $21.62 \pm 3.46$ & $<20$ & $29.14 \pm 2.06$ & $40.46 \pm 2.98$ & $10.00 \pm 1.01$ \\
\hline $\mathbf{f}$ & 30 & $30.79 \pm 2.92$ & $27.28 \pm 1.90$ & $43.28 \pm 1.53$ & $76.93 \pm 1.60$ & $23.40 \pm 0.60$ \\
\hline 27 & & & & & & \\
\hline & 10 & $24.26 \pm 2.63$ & $34.00 \pm 1.43$ & $58.38 \pm 3.20$ & $56.24 \pm 0.98$ & $25.56 \pm 2.21$ \\
\hline $\mathbf{a}$ & 30 & $85.22 \pm 1.32$ & $82.68 \pm 1.25$ & $97.21 \pm 0.10$ & $84.18 \pm 0.44$ & $99.24 \pm 0.07$ \\
\hline & 10 & $37.10 \pm 1.77$ & $39.59 \pm 1.17$ & $51.92 \pm 1.00$ & $56.44 \pm 0.98$ & \\
\hline b & 30 & $52.08 \pm 2.08$ & $69.54 \pm 1.24$ & $65.12 \pm 1.91$ & $71.81 \pm 0.96$ & \\
\hline & 10 & $38.89 \pm 2.60$ & $64.05 \pm 1.24$ & $49.68 \pm 1.66$ & $72.37 \pm 1.27$ & $13.99 \pm 1.79$ \\
\hline c & 30 & $55.93 \pm 2.39$ & $\begin{array}{c}83.34 \pm 1.31 \\
{[9.29]}\end{array}$ & $61.26 \pm 1.72$ & $\begin{array}{c}85.81 \pm 1.04 \\
{[6.74]}\end{array}$ & $29.56 \pm 1.17$ \\
\hline & 10 & $34.23 \pm 1.39$ & $30.04 \pm 2.07$ & $47.03 \pm 1.25$ & $55.77 \pm 1.03$ & \\
\hline d & 30 & $47.74 \pm 0.78$ & $39.96 \pm 2.34$ & $42.43 \pm 1.69$ & $57.71 \pm 1.00$ & \\
\hline & 10 & $<20$ & $21.53 \pm 1.81$ & $35.74 \pm 1.33$ & $<20$ & \\
\hline e & 30 & $99.06 \pm 0.09$ & $96.91 \pm 0.06$ & $98.50 \pm 0.93$ & $99.01 \pm 0.52$ & \\
\hline & 10 & $<20$ & $24.65 \pm 1.46$ & $25.50 \pm 2.93$ & $24.79 \pm 2.20$ & \\
\hline f & 30 & $98.72 \pm 0.13$ & $96.04 \pm 0.25$ & $98.41 \pm 0.15$ & $98.79 \pm 0.16$ & \\
\hline
\end{tabular}




\begin{tabular}{cc|ccccc}
\hline \multirow{2}{*}{$\mathbf{a}$} & 10 & $35.48 \pm 1.91$ & $46.07 \pm 1.13$ & $52.88 \pm 0.82$ & $25.61 \pm 2.84$ & \\
& 30 & $63.44 \pm 1.79$ & $69.86 \pm 0.55$ & $73.39 \pm 0.74$ & $52.16 \pm 2.52$ & \\
\hline \multirow{2}{*}{$\mathbf{b}$} & 10 & $39.75 \pm 2.45$ & $<20$ & $43.51 \pm 1.85$ & $44.86 \pm 0.93$ & \\
& 30 & $47.34 \pm 1.62$ & $<20$ & $42.28 \pm 1.44$ & $43.73 \pm 2.25$ & \\
& 10 & $56.71 \pm 0.57$ & $39.93 \pm 3.14$ & $48.56 \pm 0.48$ & $30.30 \pm 1.64$ & \\
$\mathbf{c}$ & 30 & $58.21 \pm 0.73$ & $31.15 \pm 2.86$ & $49.93 \pm 1.33$ & $31.60 \pm 3.08$ & \\
& 10 & $74.18 \pm 1.15$ & $76.88 \pm 0.49$ & $75.97 \pm 0.89$ & $86.12 \pm 0.33$ & $70.18 \pm 1.15$ \\
\multirow{3}{*}{$\mathbf{d}$} & 30 & $91.17 \pm 0.33$ & $87.39 \pm 0.86$ & $88.99 \pm 0.25$ & $90.72 \pm 1.00$ & $91.12 \pm 1.64$ \\
& & {$[2.30]$} & {$[4.14]$} & {$[3.87]$} & {$[3.89]$} & {$[3.71]$} \\
\hline \multirow{2}{*}{$\mathbf{e}$} & 10 & $27.42 \pm 2.16$ & $<20$ & $52.86 \pm 1.30$ & $29.58 \pm 1.69$ & \\
& 30 & $92.94 \pm 0.17$ & $91.91 \pm 0.23$ & $96.38 \pm 0.07$ & $94.09 \pm 0.43$ & \\
& 10 & $30.97 \pm 1.02$ & $39.85 \pm 1.24$ & $50.60 \pm 0.65$ & $31.89 \pm 2.92$ & \\
$\mathbf{f}$ & 30 & $91.88 \pm 0.26$ & $90.94 \pm 0.18$ & $95.12 \pm 0.10$ & $92.56 \pm 0.34$ & \\
\hline \multirow{2}{*}{ cisplatin } & 10 & $42.61 \pm 2.33$ & $86.84 \pm 0.50$ & $53.03 \pm 2.29$ & $20.84 \pm 0.81$ & $94.20 \pm 0.39$ \\
& 30 & $99.93 \pm 0.26$ & $90.18 \pm 1.78$ & $86.90 \pm 1.24$ & $74.47 \pm 1.20$ & $96.44 \pm 0.17$ \\
& & {$[12.43]$} & {$[7.84]$} & {$[5.78]$} & {$[19.13]$} & {$[3.23]$} \\
\hline
\end{tabular}



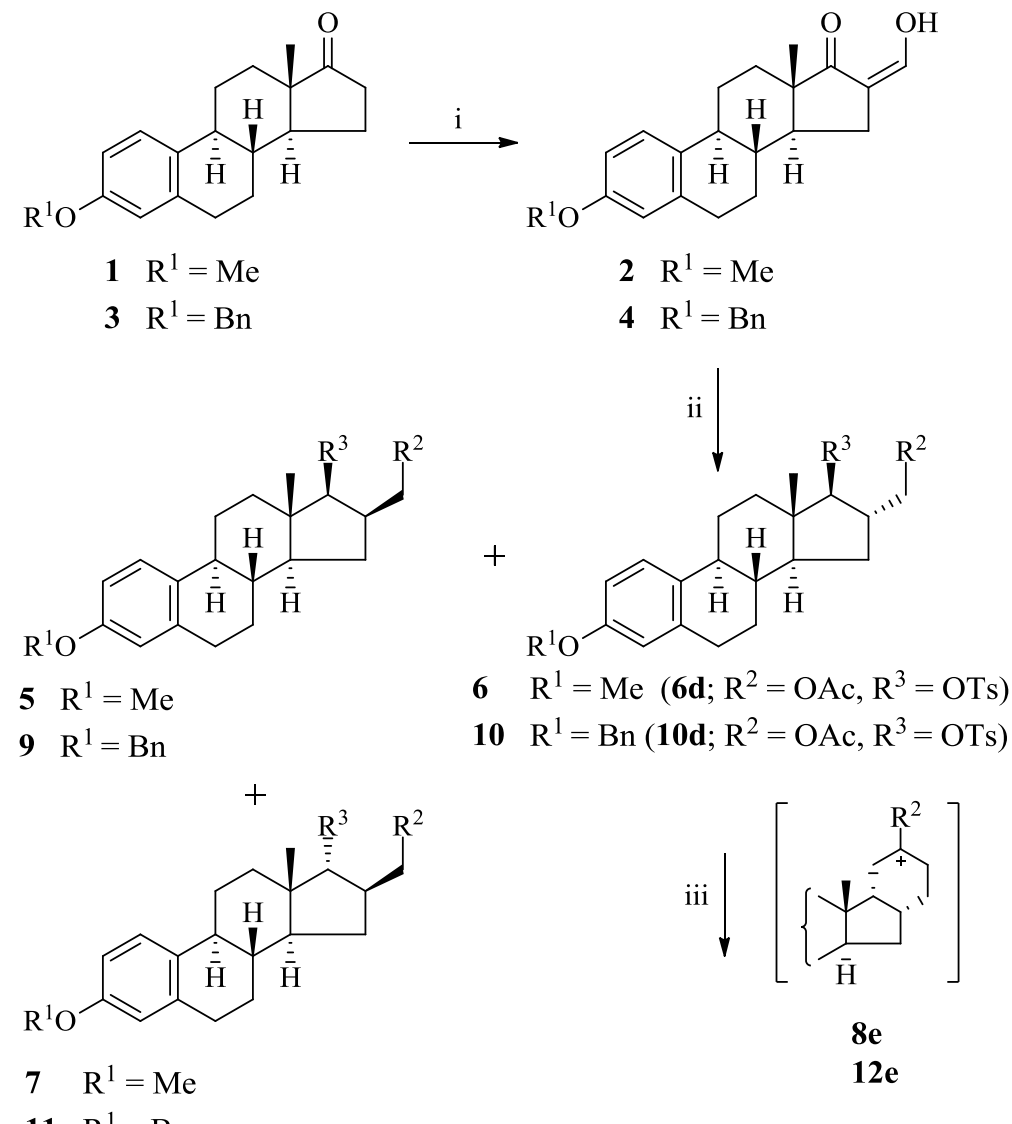

$11 \mathrm{R}^{1}=\mathrm{Bn}$

\begin{tabular}{l|l}
\hline $\mathbf{5 a}-\mathbf{1 2 a}$ & $\mathrm{R}^{2}=\mathrm{R}^{3}=\mathrm{OH}$ \\
$\mathbf{5 b}-\mathbf{8 b}$ & $\mathrm{R}^{2}=\mathrm{OTs}, \mathrm{R}^{3}=\mathrm{OH}$ \\
$\mathbf{9 c}-\mathbf{1 2 c}$ & $\mathrm{R}^{2}=\mathrm{Br}, \mathrm{R}^{3}=\mathrm{OH}$ \\
$\mathbf{6 d}, \mathbf{1 0 d}$ & $\mathrm{R}^{2}=\mathrm{OAc}, \mathrm{R}^{3}=\mathrm{OTs}$ \\
$\mathbf{8 e}, \mathbf{1 2 e}$ & $\mathrm{R}^{2}=\mathrm{Me}$
\end{tabular}

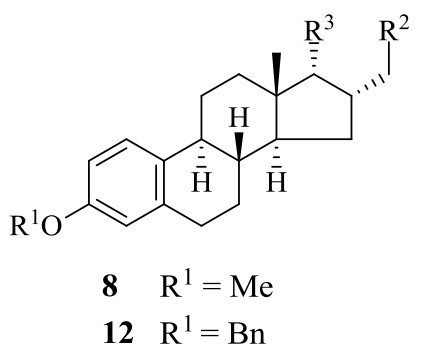

Scheme 1. Reagents and conditions: (i) NaOMe, HCOOEt, anhydrous toluene, $50{ }^{\circ} \mathrm{C}$; (ii) $\mathrm{KBH}_{4}, \mathrm{MeOH}$; (iii) $\mathrm{KOAc}, \mathrm{CH}_{3} \mathrm{COOH}, \mathrm{NaOMe} / \mathrm{MeOH}$

Scheme 1. 

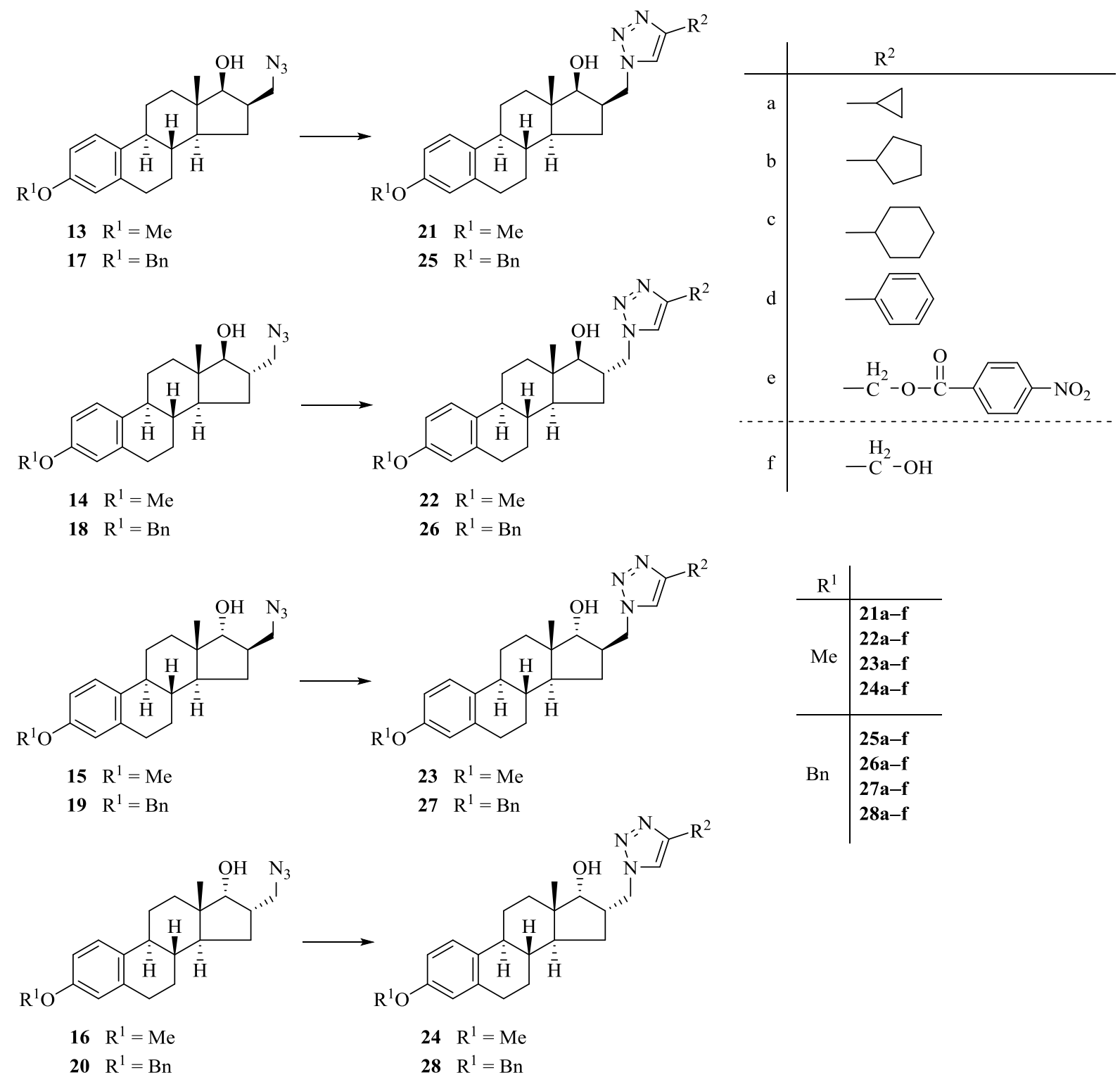

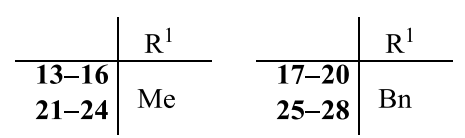

Scheme 2. 
Supplementary Material
Click here to download Supplementary Material: SCH_supporting_info_16-triazolylmethyl.pdf

Supplementary Material
Click here to download Supplementary Material: SCH_supporting_info_16-triazolylmethyl.pdf

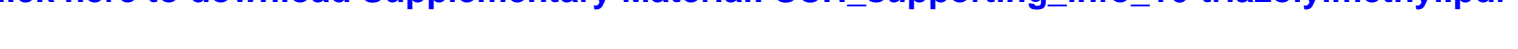

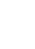

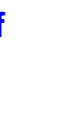

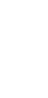

$\sqrt{10}$

$\sqrt{3}$

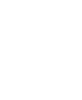

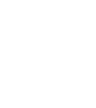

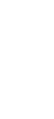

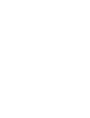

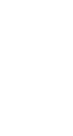

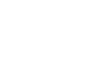

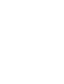
更

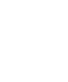

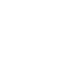

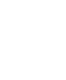

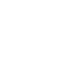

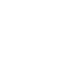

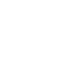

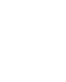
更

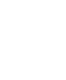
更

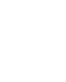

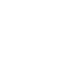

